Div. 542

NBSIR 79-1941

QEC

Table of Recommended Rate

18 is/9 Constants for Chemical Reactions Occurring in Combustion

Francis Westley

Chemical Kinetics Division

National Measurement Laboratory

National Bureau of Standards

U.S. Department of Commerce

Washington, D.C. 20234

November 1979

Prepared for

Department of Energy

Washington, D.C. 20545

and

Office of Standard Reference Data National Bureau of Standards

Washington, D.C. 20234 



\section{TABLE OF RECOMMENDED RATE CONSTANTS FOR CHEMICAL REACTIONS OCCURRING IN COMBUSTION}

Francis Westley

Chemical Kinetics Division National Measurement Laboratory National Bureau of Standards U.S. Department of Commerce Washington, D.C. 20234

November 1979

Prepared for

Department of Energy

Washington, D.C. 20545

and

Office of Standard Reference Data

National Bureau of Standards

Washington, D.C. 20234

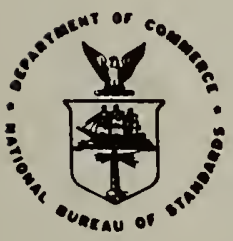

U.S. DEPARTMENT OF COMMERCE

Luther H. Hodges, Jr., Under Secretary

Jordan J. Baruch, Assistant Secretary for Science and Technology NATIONAL BUREAU OF STANDARDS, Ernest Ambler, Director 

Table of Recommended Rate Constants for Chemical

Reactions Occurring in Combustion

by

Francis Westley

Chemical Kinetics Information Center National Measurement Laboratory National Bureau of Standards Washington, DC 20234

Sponsored by the

Department of Energy Washington, DC 20545

and

Office of Standard Reference Data

National Bureau of Standards

Washington, DC 20234 


\section{Table of Contents}

Abstract . . . . . . . . . . . . 1

Introduction . . . . . . . . . . . . 2

Guidelines for the User. . . . . . . . . . 4

Sources of Recommended Rate Constants. . . . . . . .20

Table of Arrhenius parameters for chemical reactions occurring in combustion ...........22

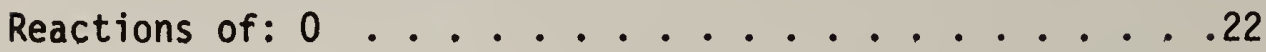

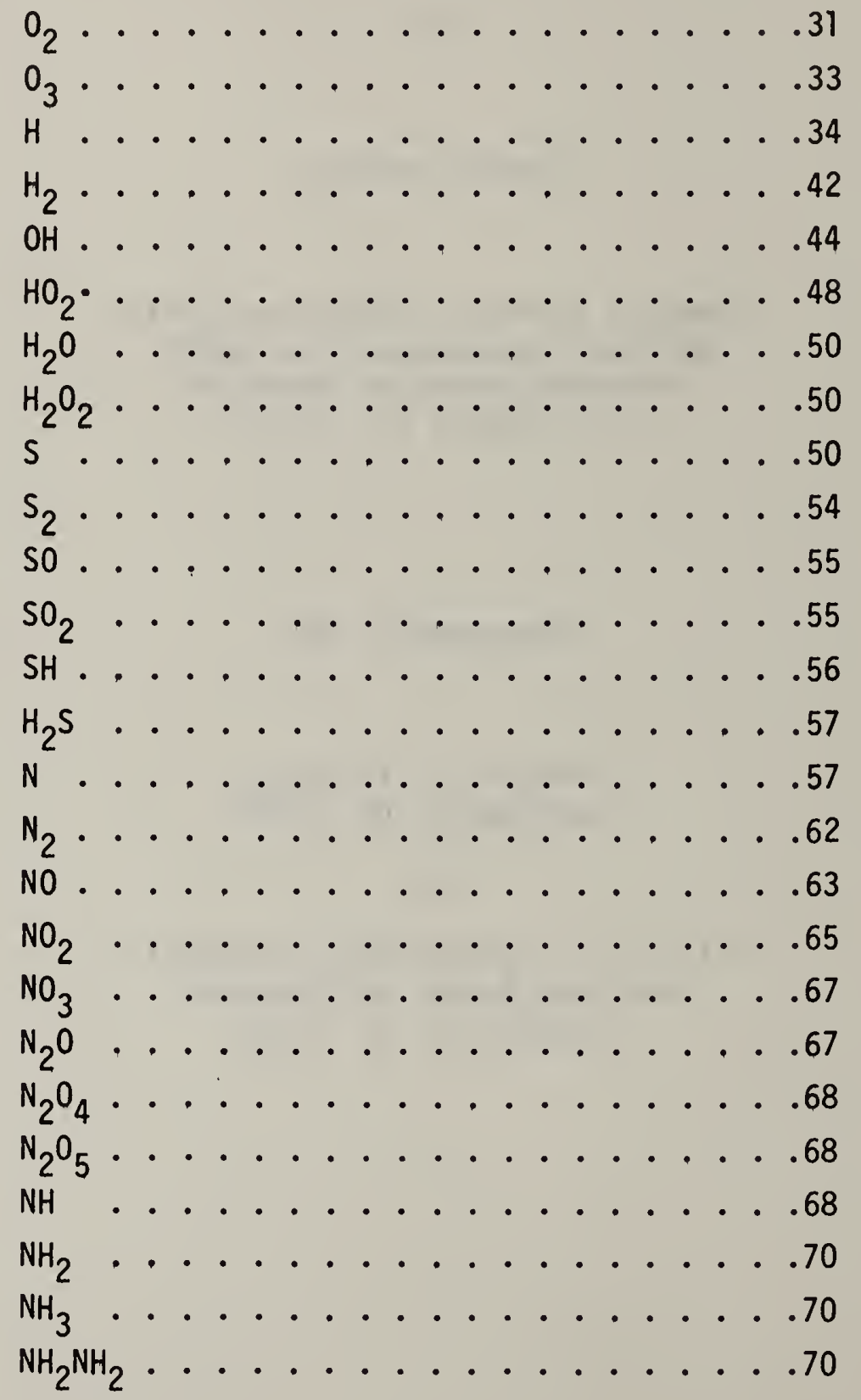




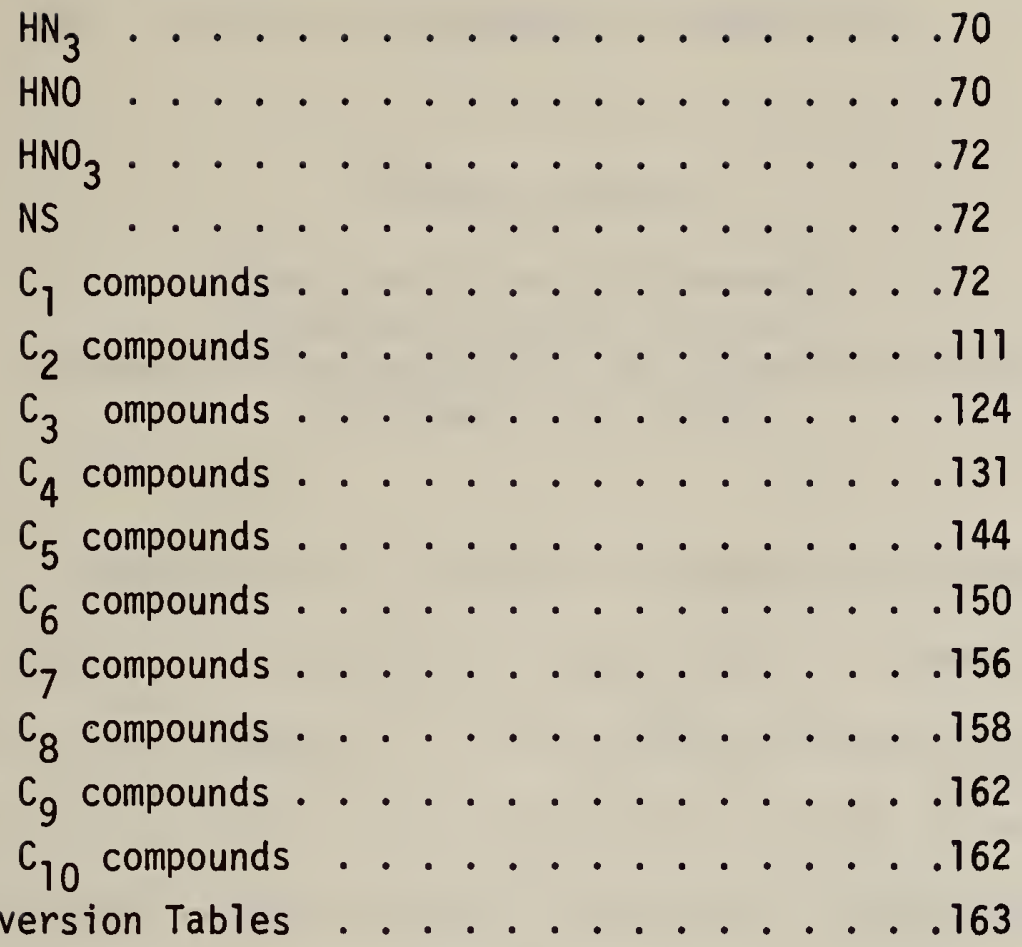

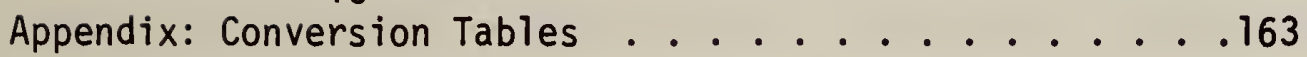





\title{
TABLE OF RECOMMENDED RATE CONSTANTS FOR CHEMICAL * REACTIONS OCCURRING IN COMBUSTION
}

\author{
Francis Westley \\ National Bureau of Standards \\ Chemical Kinetics Division \\ Center for Thermodynamics and Molecular Science \\ Washington, DC 20234
}

A table of recommended rate constants for gas phase chemical reactions occurring in combustion is presented. Specifically, it gives in tabular form the values of the parameters for the modified Arrhenius equation $k=A T^{B} \exp (-E / R T)$. The table covers reactions occurring in the combustion, oxidation and decomposition of aliphatic saturated or unsaturated $C_{1}$ to $C_{10}$ hydrocarbons, alcohols, aldehydes, ketones, thiols, ethers, peroxides, amines, amides and their free radicals, as well as the reactions of $0, \mathrm{O}_{2}, \mathrm{H}, \mathrm{H}_{2}, \mathrm{OH}$, $\mathrm{H}_{2} \mathrm{O}, \mathrm{H}_{2} \mathrm{O}_{2}, \mathrm{~N}, \mathrm{~N}_{2}, \mathrm{NO}, \mathrm{N}_{2} \mathrm{O}, \mathrm{NO}_{2}, \mathrm{~N}_{2} \mathrm{O}_{4}, \mathrm{~N}_{2} \mathrm{O}_{5}, \mathrm{~S}, \mathrm{~S}_{2}, \mathrm{SH}$, SO, $\mathrm{SO}_{2}, \mathrm{SOH}$, NS, with each other. The table includes 169 first order reactions 782 second order reactions and 57 third order reactions. There are 1770 entries covering 1008 distinct chemical reactions. These recommendations have been taken from eleven evaluations and critical reviews published between 1970 and 1976. The papers examined by the evaluators extend from the nineteen fifties up to - and including - 1975.

Keywords: Arrhenius parameters, chemical kinetics, combustion, decomposition, free radicals, gas phase, hydrocarbons, hydrogen, nitrogen, oxygen, rate of reaction, sulfur.

This work was supported by the Department of Energy and by the Office of Standard Reference Data of the National Bureau of Standards. 
This publication consists of a table of recommended reaction rate constants for the combustion, oxidation and decomposition reactions of aliphatic saturated and unsaturated hydrocarbons, their oxygenated sulfur and amino derivatives, as well as for the reactions of hydrogen, nitrogen, oxygen, sulfur and their inorganic derivatives with each other. The table is a compilation of recommended rate constants given in eleven critical reviews on the kinetics of combustion, oxidation and decomposition reactions, published between 1970 and 1976. Its purpose is to provide the kineticists and kinetic modelers with a comprehensive and easy-to-consult reference book on the kinetic data for combustion and oxidation processes. The table gives 1008 recommended reaction rate constants from these eleven sources. A summary of the content of the table is given in the following listing of quotations from each source.

\section{SOURCE}

BAULCH et al (1972)

BAULCH et al (1973)

BAULCH et al (1976)

BENSON and O'NEAL (1970)

BENSON et al (1975)

ENGLEMAN (1976)

HERRON and HUIE (1973)

KERR and PARSONAGE (1972)

KERR and PARSONAGE (1976)

KONDRATIEV (1970)

LLOYD (1974)

\section{\# RECOMPIENDED RATE CONSTANTS}

\begin{tabular}{rr}
40 \\
64 \\
36 \\
167 \\
119 \\
123 \\
46 \\
185 \\
181 \\
37 \\
10 \\
\hline TOTAL & 1008
\end{tabular}

For ease of reference the bimolecular and termolecular reactions included in the table are listed separately under each reactant, so that a grouping 
of the reaction according to the first reactant is obtained. As a result, the total number of tabulated entries is 1770 , although the real number of distinct chemical reactions is 1008 .

The presentation of kinetic data is standardized and simplified as much as possible. Rate constants are expressed in the modified Arrhenius equation $k=A T^{B} \exp (-E / R T)$. In general uncertainties are given only for the rate constant $k$ itself and not for the individual parameters in this equation. Sometimes an uncertainty is given for the value of $E / R$. This uncertainty is only of secondary importance and has been included in the uncertainty stated for the value of the rate constant. Rate constants are expressed in units of $\mathrm{s}^{-1}, \mathrm{~cm}^{3} \mathrm{~mol}^{-1} \mathrm{~s}^{-1}$, and $\mathrm{cm}^{6} \mathrm{~mol}^{-2} \mathrm{~s}^{-1}$ for reactions of first, second and third order respectively.

For the readers who prefer other kinetic units than the standard ones, two conversion tables for equivalent second and - respectively - third order rate constant units are appended at the end of this publication.

The arrangement of the tables is described in detail below, in the "Guidelines for the User".

It is hoped that this table of kinetic data will serve as a handy and easy to use reference book for all the kineticists and kinetic modelers interested in combustion and oxidation processes.

This publication is not the result of the effort of a single person, but of the whole staff of Chemical Kinetics Information Center. My thanks to all of them.

In particular, I wish to thank Dr. David Garvin, Chief of the Chemical Thermodynamics Division, and Dr. Robert F. Hampson, Jr., Director of the Chemical Kinetics Information Center, for their more than helpful suggestions and constant guidance; Dr. Wing Tsang, Chief of the Chemical Kinetics Division, for his encouragement in having this table published; 
Mr. James G. Koch, Supervisor, for putting the tables into a printable computer form; Mrs. Bettijoyce Molino and Mrs. Carla G. Messina from the Office of Standard Reference Data for applying the OMNIDATA and GPSDIC programs to the present tables; Mrs. Geraldine Zumwalt and Mrs. Janice L. Jones for punching and typing a difficult typescript, full of digits and numbers, with particular care.

\section{GUIDELINES FOR THE USER}

\section{General}

As pointed out above, the presentation of the kinetic data in this publication is an attempt to simplify and standardize them. In that respect, the choice of standard units for rate constants was easy; it was found that the most commonly used units for gas phase rate constants are the cubic centimeter, the mole and the second. The choice of a standard form for uncertainty limits is somewhat more complicated, but when a series of recommended rate constants is to be presented in a tabular form, the uncertainty limits can not be omitted, for an uncertainty assigned to the recommended value of a rate constant is an estimate by the evaluator of the absolute accuracy of the preferred value. It is to be emphasized that in the present tables the concern is with the overall uncertainty of a reaction rate constant and not with the expression of precision of a set of experimental measurements. Most of the uncertainty limits included in this table are uniform within the respective temperature range indicated. However, for a limited number of reactions, the data warrant or require variable limits. In such cases, a note under the respective data indicates for which interval of the temperature range there is a change in the uncertainty limits.

It is thought that the uncertainty limits expressed in the form of lower and upper $k$ factors - $f$ and $F$, respectively - are the most suitable for 
tabulation. Thus, if $k_{0}$ is the central value of a rate constant the limits of reliability for the rate constant $k_{0}$ are defined by the relationship:

$$
f k_{0}<k<F k_{0}
$$

i.e. multiplication of the central value $k_{0}$ by $f$ and $F$ gives respectively the lower and upper reliability limits of the rate constant. In this standardized formulation of uncertainty limits, the value of $f$ is less than unity and the value of $F$ is greater than unity.

However the $k$ factors are not the only way to express the uncertainty limits of a rate constant and different authors use different forms to indicate the degree of reliability of a recommended rate constant. It follows that certain mathematical relationships are needed to translate the different forms of uncertainty limits into the standard form used in this table (lower and upper $k$ factors). The transformation formulas are given and discussed below.

In general there are two ways to state uncertainty limits: 1) by factors and by algebraic addends

\section{Uncertainty expressed by factors.}

Beside the standard form of uncertainty limits expressed by the lower and upper factors $f$ and $F$, as defined by the above given relationship (1), there is another form which expresses the uncertainty limits by a unique factor. Thus, if $k_{0}$ is the central value of a rate constant, the statement that $k_{0}$ is uncertain to a factor of $F$ means that the uncertainty limits are defined by the relationship:

$$
k_{0} / F<k<k_{0} F
$$

which shows that division and multiplication of the central value $k_{0}$ by $F$ gives respectively the lower and upper reliability limits of the rate 
constant. By comparing relationships (1) and (2), it is obvious that in the case of an uncertainty expressed by an unique $F$ the upper factor is equal to the unique factor itself, while the lower factor $f$ is the reciprocal of $F$ :

$$
f=1 / F
$$

\section{Uncertainty expressed by algebraic addends.}

There are three types of uncertainty limits for rate constants expressed as algebraic addends, which are currently used by kineticists: a). Uncertainty appended to one of the Arrhenius factors ( $A, B$, or $E / R$ ); b). Uncertainty appended to $\log 10 k_{0} ;$ and $\left.c\right)$. Uncertainty expressed as a percentage of $k_{j}$ With respect to the type a) uncertainties, the $B$ factor uncertainties have been eliminated as being unimportant, while the uncertainties for the $E / R$ factor may be omitted because they are of secondary importance and are included in the $k$ factors. Therefore, the only uncertainty of type a) considered below is the one appended to the coefficient of the $A$ factor.

a). Uncertainty appended to the coefficient of A factor. In scientific notation, the A factor is of the form:

$$
A=\operatorname{ax} 10^{n}
$$

where a is a numerical coefficient less than 10 and $n$ is the power of 10 . If an uncertainty $\pm a^{\prime}$ is appended to the coefficient a, the A factor takes the form:

$$
A=\left(a \pm a^{\prime}\right) \times 10^{n}
$$

If lower and upper factors ( $f$ and $F$ ) are wanted instead, the A factor takes the form:

$$
\begin{aligned}
& A & =f a \times 10^{n} \\
\text { or } & A & =F a \times 10^{n}
\end{aligned}
$$


Comparison of $(6)$ and $(7)$ to (5) leads to the relationships: $f a=a-a^{\prime}$ and $\mathrm{Fa}=\mathrm{a}+\mathrm{a}^{\prime}$ from which the following formulas are obtained:

$$
\text { and } \quad \begin{aligned}
f & =1-a^{\prime} / a \\
& F=1+a^{\prime} / a
\end{aligned}
$$

Formulas (8) and (9) are the relationships needed to transform an uncertainty appended to the coefficient of the $A$ factor into one using a $k$ factor. A numerical example follows:

$$
\begin{array}{r}
A=(2.0 \pm 0.5) \times 10^{14} \text { therefore: } a=2.0 \text { and } a^{\prime}=0.5 \\
a^{\prime} / a=0.5 / 2.0=0.25 \text { and the } k \text { factors are: } f=1-0.25=0.75 \\
\text { and } F=1+0.25=1.25
\end{array}
$$

b). Uncertainty appended to $\log _{10 k_{0}}$. If $k_{0}$ is the central value of a rate constant, $C$ its logarithm to the base 10 and $D$ the uncertainty expressed as an algebraic addend to $C$, then the following relationship is true:

$$
\log _{10} k=C \pm D
$$

where $C=\log _{10} k_{0}$. If $D$ is put in logarithmic form, say:

$$
D=\log _{10} F
$$

then relationship (10) becomes:

$$
\log _{10} k=\log _{10} k \pm \log _{10} F
$$

which can take the form:

$$
\log _{10} \mathrm{k}_{\mathrm{o}} / \mathrm{F}<\log _{10} \mathrm{k}<\log _{10} \mathrm{Fk}
$$

or

$$
\mathrm{k}_{\mathrm{o}} / \mathrm{F}<\mathrm{k}<\mathrm{Fk}_{0}
$$

Replacing $1 / F$ by $f$, relationship ( 1 ) is obtained. It is obvious that the $k$ factors $f$ and $F$ are the antilogarithms of $-D$ and $D$, respectively:

$$
\begin{array}{ll}
f=\operatorname{antilog}(-D) & =10^{-D} \\
F=\operatorname{ant} i \log D & =10^{D}
\end{array}
$$


Formulas (14) and (15) are the relationships needed to transform the type b uncertainties into reliability limits expressed by $k$ factors. A numerical example follows:

$\log _{10} k=14.23 \pm 0.3$ therefore: $f=10^{-0.3}=0.5$ and $F=10^{0.3}=2.0$

c). Uncertainty expressed in percentage of $k_{0}$. Some kineticists prefer to use percentage for defining the uncertainty limits of a rate constant. Thus, the statement that a rate constant is $\pm p \%$ uncertain means that the uncertainty limits of $k_{0}$ are defined by the relationship:

$$
\begin{aligned}
& k_{0}-(p / 100) k_{0}<k<k_{0}+(p / 100) k_{0} \\
\text { or } \quad & (1-p / 100) k_{0}<k<(1+p / 100) k_{0}
\end{aligned}
$$

Replacing the percentage by the rate, defined as $r=p / 100$, relationship (16) becomes:

$$
(1-r) k_{0}<k<(1+r) k_{0}
$$

Comparison of relationships (17) and (1) leads to the following formulas:

$$
\text { and } \begin{aligned}
f & =1-r \\
& F=1+r
\end{aligned}
$$

which are the relationships needed to transform the type c uncertainties into reliability limits expressed by $k$ factors. A numerical example foll lows:

$$
\begin{gathered}
k=3.7 \times 10^{12} \pm 20 \% \text { therefore } p=20 \% \text { and } r=0.2 \\
\text { Thus: } f=1-0.2=0.8 \\
\text { and } F=1+0.2=1.2
\end{gathered}
$$

When a percent error has been stated as $>100 \%$, the $F$ factor is determined first, according to relationship (19) then, instead of relationship (18), one simply sets: $f=1 / F$. E.g.: for a $150 \%$ error, $r=1.5, F=1+r=$ 2.5 and $f=1 / F=0.4$. 
The above given relationships: (3), (8) and (9), (14) and (15), (18) and (19) can be used in reverse by the reader who prefers other types of uncertainty limits than the standard $k$ factors, $f$ and $F$. However a word of caution is necessary. In contrast with the standard uncertainty limits, other types of uncertainties for rate constants using a unique factor or algebraic addend have a constraint imposed upon them. Thus, the uncertainties expressed by a unique algebraic addend are required to be symmetrical with respect to the central value to which they are appended, while the uncertainty expressed by a unique factor, $F$, indicates in fact that the upper factor $F$ and the lower factor $f$ are required to be inverse to each other $(f=1 / F)$. No such constraints are imposed on the standard uncertainty limits used here and for that reason this type of uncertainty has been found most suitable for tabulation purposes. Why some evaluators prefer uncertainty limits with constraints is not clear. It would seem more logical if the lower and upper uncertainty limits were studied each independently from each other, without imposing any constraints on them. Probably it is a matter of convenience to express an uncertainty in the form - say - : $\log _{10} k=C \pm D$ rather than by the inequality: $\mathrm{fk}_{\mathrm{o}}<\mathrm{k}<\mathrm{Fk}_{\mathrm{o}}$.

If the transformation of the standard uncertainty limits into uncertainties with constraints is desired, some adjustments may be necessary according to the case. The following examples, for transformation of standard uncertainty into a unique factor uncertainty, are an illustration of the necessary adjustments :

1). Standard factors: $f=0.5$ and $F=2.0$ it is obvious that $f=1 / F$ and no adjustment is necessary.

2). Standard factors: $f=0.8$ and $F=1.2$ In this case, $f$ and $F$ are not inverse to each other. Indeed $f^{\prime}=1 / F=0.83$ while $F^{\prime}=1 / f=1.25$. 
The two pairs of factors, $\left(f^{\prime}=0.83 ; F=1.2\right.$ and $\left.f=0.8 ; F^{\prime}=1.25\right)$ are quite close. However, it is safer to choose the pair 0.8 and 1.25 , by enlarging slightly the uncertainty range.

3). Standard factors: $f=0.6$ and $F=1.4$ In this case, not only the factors are not inverse to each other, but the difference is significant: $f^{\prime}=1 / F=0.71$ and $F^{\prime}=1 / f=1.67$.

The two pairs of factors, $\left(f^{\prime}=0.71 ; F=1.4\right.$ and $\left.f=0.6 ; F^{\prime}=1.67\right)$ are significantly different. Again, it is safer to choose the pair 0.6 and 1.67, by enlarging the uncertainty range. And, since the concepts of uncertainty and reliability are opposite to each other, enlargement of the uncertainty range will result in a decrease in reliability.

The same adjustments may be necessary for transformation of $k$ factors uncertainties into another type of uncertainties.

\section{Arrangement of the table}

This publication is in two parts:

Part 1. The table, arranged in six columns including the chemical reactions, temperature range, the parameters $A, B$ and $E / R$ for the modified Arrhenius equation $k=A T^{B} \exp (-E / R T)$ and the uncertainty limits expressed as $k$ factors $f$ and $F$.

Part II. The bibliography of part I, including the full references for the 11 critical reviews from which the present table was compiled. Following the bibliography, two conversion tables for equivalent second and - respectively - third order rate constant units are appended.

Column 1 includes the chemical reactions indicating both the reactants and the products. In the same column, under each chemical reaction, the names of the reactants are given. The chemical nomenclature adopted is the one used in the Chemical Substance Indexes of Chemical Abstracts. 
Alternative neames are not given. The chemical names of the products are not given. The line with chemical names is indented with respect to the line above it. Under the chemical names, the short reference of reviewer's book or article is given. It includes the last two digits of publication's year, followed by the first three letters of author's name. If two authors are given, a slash separates each author's three letters. Again, the short reference line is indented with respect to the line above it. E.g.:

$$
\begin{aligned}
& 73 \text { HER/HUI indicates the review of rate constants for the } \\
& \text { reactions between aliphatic hydrocarbons and } \\
& \text { atomic oxygen, published by Herron and Huie } \\
& \text { in } 1973 \text {. }
\end{aligned}
$$

In the same line with the short reference, but spaced out, the order of reaction is indicated by the words "Reaction order:" followed by one of the digits 1,2 , or 3 . As pointed out in the introduction, the order of reaction helps to establish the proper standard units for the reactions, as follows:

$$
\begin{aligned}
& 1 \text { for first order reactions } \ldots \ldots \ldots \ldots \ldots \ldots \ldots \mathrm{s}^{-1} \\
& 2 \text { for second order reactions } \ldots \ldots \ldots \ldots \mathrm{cm}^{3} \mathrm{~mol}^{-1} \mathrm{~s}^{-1} \\
& 3 \text { for third order reactions } \ldots \ldots \ldots \ldots \mathrm{cm}^{6} \mathrm{~mol}^{-2} \mathrm{~s}^{-1}
\end{aligned}
$$

Following the reaction order, -on the same line-, the presence of an inert reaction partner ("third body") is indicated by the letter M: followed by its chemical formula. E.g.: $\mathrm{M}: \mathrm{Ar}$ or $\mathrm{M}: \mathrm{CO}_{2}$. No indication is given if $M$ is undefined, or if the reaction does not include $M$. In all, there are 112 reactions with M specified.

For 124 reactions, no Arrhenius parameters are indicated. Instead, for each of these 124 reactions, the ratio of the rate constant with 
respect to the rate of a reference reaction - taken as unity - is given.

This information follows the reaction order information, on the same line, and is indicated by the symbol $k / k_{\text {ref }}$ : followed by a number.

$$
\text { E.g.: } k / k_{r e f}: 0.59 \text {. }
$$

The last line of column 1, placed under the line including the short reference and reaction order information, begins with the heading NOTE:. It is given only when necessary and might include information about the dependence of $k$ factors on temperature range, or the reaction taken as reference when the ratio $k / k_{r e f}$ is given in the previous line, or other information pertinent to the reaction indicated above. The rate constant, $k_{\text {ref }}$, for the reference reaction indicated in the note (by the same author) can be found in the table in the proper place. For a certain number of reactions taken from Baulch, et al. (1972, 1973, and 1976) the relationship $k_{1}=k k_{-1}$ included in the note indicates that the respective rate constant was calculated from the equilibrium constant $k$ and the rate constant $k_{-1}$ of the reverse reaction. In such cases, the author usually gives the rate constant of the reverse reaction immediately after the data for the forward reaction. The arrangement of the present table (based on the standard order, as described below in the following paragraph) does not allow the forward rate constant of a reaction to be followed immediately by its reverse reaction data. The reader will have to locate the rate constant of a reverse reaction (by the same author) in its proper place in the table.

Column 2, with the heading $T / K$, indicates in degrees Kelvin the temperature range of validity of the recommended rate parameters. For some reactions only one temperature is given, meaning that the reaction was 
studied only at one temperature. If no temperature at all is indicated, it means that the kinetic parameters of the corresponding reaction are valid throughout the normal temperature range for combustion. The data estimated by Benson and Golden in their report "Estimating the Kinetics of Combustion" are in this category. The temperatures are alined with the short reference and the reaction order information.

Column 3, with the heading $A$, gives the value of $A$ for the equation $k=A T^{B} \exp (-E / R T)$ in short scientific notation. In other words, it appears as a number less than 10, followed by a parenthesis including an integer preceded by the sign + , or - . The number less than 10 is the coefficient of the $A$ factor, while the integer inside the parenthesis is the exponent of 10. Therefore, e.g., $3.5(+14)$ should be read as $3.5 \times 10^{+14}$. The coefficient of the A factor has no more than one digit after the decimal point. The units of the A factor are the same as for the rate constant $k_{1}$ according to the order of the respective reaction, as shown above on page 11 of this introduction. For those cases when the recommended value is only for one temperature, the entry under this column is in fact the value of the rate constant $k$ at this temperature. As for the temperatures, the data for the A factor are alined with the short reference and reaction order information. If a dash appears in this column, it means that no A factor value was reported by the evaluator for the corresponding reaction.

Column 4, with the heading $B$, gives the value of $B$ for the equation $k=A T^{B} \exp (-E / R T)$. The value of $B$ is usually low and varies from 0 to about 3 or 4 . It may be negative, or positive. The negative values of B are preceded by the sign -, while the positive values are without sign. No more than one digit is given after the decimal dot. If' in this column 
a dash appears instead of a figure, it means that no B value was reported by the evaluator for the corresponding reaction. As for the temperature and $A$ factor, the data for $B$ are alined with the short reference and reaction order information.

Column 5, with the heading $E / R$, indicates the value of $E / R$ for the equation $k=A T^{B} \exp (-E / R T)$. Since $E$ is the activation energy in cal mol ${ }^{-1}$ and $R$ the gas constant with a value of $1.987 \mathrm{cal} \mathrm{mol}^{-1} \mathrm{~K}^{-1}$, it follows that the units of $E / R$ are degrees $K$. The values given in column 5 for $E / R$ may vary from 0 to over 100000 degrees $K$. The $E / R$ values may be positive or negative. The negative values are preceded by - , while the positive values are without sign. Some of the $E / R$ values included in the table are followed by an uncertainty with plus or minus sign. As pointed out in the introduction, these uncertainties may be ignored, as being included in the lower and upper $k$ factors indicated in the right column of the table. If in this column a dash appears instead of a figure, it means that no $E / R$ value was reported by the evaluator. As for the data from the previous columns, the values for the E/R factor are alined with the short reference and reaction order information.

Column 6, with the heading "k factors" and two subheadings, "f"l and " $F$ ", indicates the two uncertainty $k$ factors, the lower factor $f$ in the left subcolumn and the upper factor $F$ in the right subcolumn. To find the uncertainty limits of a reaction, its rate constant is to be multiplied by the two factors, as shown above in relation (1): $\mathrm{fk}_{0}<\mathrm{k}<\mathrm{Fk}_{0}$. The values of both factors are always positive. If no uncertainty limits are indicated by the evaluator, both subcolumns of the column 6 are left blank. As for the data from the previous columns, the $k$ factors are alined with the short reference and reaction order information. 
The general rule for ordering the chemical equations of the reactions listed in column 1 of the table is the standard order of arrangement as described in NBS Technical Note 270-3 pp. 5, 6, and 22\%). This rule is applied to the first reactants of the reactions listed in the table, as well as to the reactants following the first. The first reactant of a reaction takes precedence over the following ones. The compounds $l$ isted as reactants may include the atoms $\mathrm{O}, \mathrm{H}, \mathrm{S}, \mathrm{N}$, and $\mathrm{C}$, either each of them separately, or several, in any possible combination. The standard order of arrangement, when applied to these five atomic species, will result in the sequence $0, H, S, N, C$, each atom in it taking precedence over the following ones. When applied to the first reactants listed in the table, the standard order of arrangement will result in a sequence of five chemical systems, whose order of precedence is as follows:

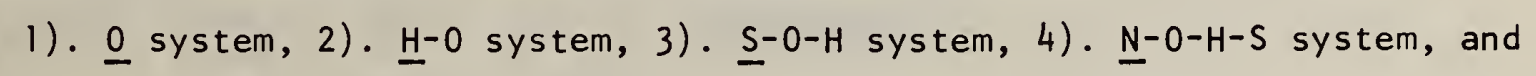
5). $\underline{C}-0-H-S-N$ system.

In each system, the first atom is underlined to show that the compounds containing this atom only, should be listed first. It is to be noted that the atomic species following the underlined atom are in standard order, while the underlined atom itself should be put at the end if the standard order were to be followed. As it will be shown below, this exception to the standard rule, - which is apparent only but not real, - is due to

*). Wagman, D. D., Evans, W. H., Parker, V. B., Halow, I., Bailey, S. M., and Schumm, R. H., "Selected Values of Chemical Thermodynamic Properties," NBS Tech. Note 270-3 pgs. 5, 16, 22 (1968). 
the fact that all the compounds containing the atoms of a system with the exception of the underlined atom, are already listed in the previous

systems. In each of these five chemical systems, the order of the compounds listed in the table as first reactants is as follows:

1). 0 system: $0, \mathrm{O}_{2}, \mathrm{O}_{3}$.

2). $\mathrm{H}-\mathrm{O}$ system: $\mathrm{H}, \mathrm{H}_{2}, \mathrm{OH}, \mathrm{HO}_{2}, \mathrm{H}_{2} \mathrm{O}, \mathrm{H}_{2} \mathrm{O}_{2}$.

3). S-O-H system: $\mathrm{S}, \mathrm{S}_{2}, \mathrm{SO}, \mathrm{SO}_{2}, \mathrm{SO}_{3}, \mathrm{SH}, \mathrm{SH}_{2}, \mathrm{SOH}$.

4). N-O-H-S system: $\mathrm{N}, \mathrm{N}_{2}, \mathrm{NO}, \mathrm{NO}_{2}, \mathrm{NO}_{3}, \mathrm{~N}_{2} \mathrm{O}, \mathrm{N}_{2} \mathrm{O}_{4}, \mathrm{~N}_{2} \mathrm{O}_{5}, \mathrm{NH}, \mathrm{NH}_{2}$, $\mathrm{NH}_{3}, \mathrm{~N}_{2} \mathrm{H}_{4}, \mathrm{HN}_{3}$, HNO, HNO 3 , NS.

5). C-O-H-S-N system: $\mathrm{C}_{1}$ compounds: $\mathrm{C}, \mathrm{CO}, \mathrm{CO}_{2}, \mathrm{CH}, \mathrm{CH}_{2}, \mathrm{CH}_{3}, \mathrm{CH}_{4}, \mathrm{CHO}$, $\mathrm{HCHO}, \mathrm{CH}_{3} \mathrm{O}, \mathrm{CH}_{3} \mathrm{OH}, \mathrm{CH}_{3} \mathrm{OOH}, \mathrm{CS}, \mathrm{CS}_{2}$, $\mathrm{COS}, \mathrm{CH}_{3} \mathrm{~S}, \mathrm{CH}_{3} \mathrm{SH}, \mathrm{CN}, \mathrm{C}\left(\mathrm{NO}_{2}\right)_{4}, \mathrm{CHN}$, $\mathrm{CH}_{3} \mathrm{NH}_{2}, \mathrm{CH}=\mathrm{N} \equiv \mathrm{N}, \mathrm{CH}_{3} \mathrm{NHNH}_{2}, \mathrm{CH}_{3} \mathrm{NO}$, $\mathrm{CH}_{3} \mathrm{NO}_{2}, \mathrm{CH}_{3} \mathrm{NO}_{3}, \mathrm{CH}_{3} \mathrm{ONH}_{2}$.

$C_{2}$ compounds: $\mathrm{C}_{2}, \mathrm{C}_{2} \mathrm{O}, \mathrm{CH} \equiv \mathrm{CH}, \mathrm{CH}_{2}=\mathrm{CH}_{2}, \mathrm{CH}_{3} \mathrm{CH}_{2} \cdot$, $\mathrm{CH}_{3} \mathrm{CH}_{3}, \mathrm{CH}_{2}=\mathrm{C}=0$, etc.

$\mathrm{C}_{3}$ compounds, etc., up to $c_{10}$ compounds follow, being ordered according to the same pattern.

It is clear now that, for instance, the compounds included in the $\underline{S}-0-H$ system contain at least one sulfur atom, while the compounds containing only $H$, or 0 atoms, or both, are already listed in the previous two systems (0-system and $\underline{H}-0$ system). It is to be noted that for the $\underline{C}-0-H-S-N$ system the standard order is applied in a slightly different way: the compounds are first grouped according to the number of $C$ atoms, then the rule for the standard order of arrangement is applied for each group apart. This is necessary as a result of the very large number of organic compounds.

The standard order is applied in the same way for the second, or third reactants of chemical reactions. Since the reactants of a chemical equation 
can be switched around, a number of bimolecular and termolecular reactions are inserted in the table in two - and respectively - three places. E.g.: Reaction $\mathrm{CH}_{4}+\mathrm{O} \rightarrow \mathrm{CH}_{3} \cdot+\mathrm{OH}$, is inserted in the $\underline{\mathrm{C}}-\mathrm{O}-\mathrm{H}-\mathrm{S}-\mathrm{N}$ system. This reaction may also be written as $\mathrm{O}+\mathrm{CH}_{4} \rightarrow \mathrm{OH}+\mathrm{CH}_{3}$ and, as such, is listed in the $\underline{0}$ system. The advantage of such a procedure is obvious: referring to the example just given the reader will find the reaction between methane and oxygen listed with $\mathrm{CH}_{4}$ as first reactant if he is interested in the reactions of methane, or listed with 0 as first reactant, if he is interested in the reactions of oxygen atom. The bimolecular reactions are the largest group of reactions included in the table. There are about 750 reactions 1 isted in the table, having as reactants two distinct chemical compounds. Since each of these reactions is inserted twice, the number of entries for them will amount to about 1500 . Only a small number of termolecular reactions has three distinct reactants. As an example, one of them is $\mathrm{NO}+\mathrm{NO}_{2}+\mathrm{O}_{2} \rightarrow$ $\mathrm{N}_{2} \mathrm{O}_{5}$. This reaction will also be inserted under the forms: $\mathrm{NO}_{2}+\mathrm{NO}+\mathrm{O}_{2} \rightarrow$ $\mathrm{N}_{2} \mathrm{O}_{5}$ and $\mathrm{O}_{2}+\mathrm{NO}+\mathrm{NO}_{2} \rightarrow \mathrm{N}_{2} \mathrm{O}_{5}$. A number of second and third order reactions includes a second and - respectively - third body M. For this group of reactions, $M$ will always be placed after all the other reactants, which means that the second order reactions with $M$ as reactant will be inserted in the table only once, while the third order reactions with $M$ as reactant will be inserted only twice. E.g.: Reaction $\mathrm{O}_{3}+M \rightarrow 0+\mathrm{O}_{2}+M$ is inserted in the table only once, while reaction $\mathrm{NO}+\mathrm{O}+\mathrm{M} \rightarrow \mathrm{NO}_{2}+\mathrm{M}$ is inserted as such, and also under the form $\mathrm{O}+\mathrm{NO}+\mathrm{M} \rightarrow \mathrm{NO}_{2}+\mathrm{M}$.

Most of the chemical reactions included in the table are balanced. A number of reactions are only apparently unbalanced. For instance, reaction

$$
\mathrm{CH}_{3} \mathrm{CH}_{2} \mathrm{CH}_{2} \mathrm{CH}_{3}+\mathrm{H}+\mathrm{CH}_{3} \mathrm{CH}_{2} \mathrm{CH}_{2} \mathrm{CH}_{2} \cdot+\mathrm{CH}_{3} \mathrm{CH}_{2} \mathrm{CH}(\cdot) \mathrm{CH}_{3}+\mathrm{H}_{2}
$$

has a rate constant which in fact the sum of the rate constants for the two reactions 


$$
\text { and } \begin{aligned}
\mathrm{CH}_{3} \mathrm{CH}_{2} \mathrm{CH}_{2} \mathrm{CH}_{3}+\mathrm{H} & \rightarrow \mathrm{CH}_{3} \mathrm{CH}_{2} \mathrm{CH}_{2} \mathrm{CH}_{2} \mathrm{CH}_{3}+\mathrm{H} \rightarrow \mathrm{CH}_{3} \mathrm{CH}_{2} \mathrm{CH}(\cdot) \mathrm{CH}_{3}+\mathrm{H}_{2}
\end{aligned}
$$

Since the Arrhenius parameters listed in the table refer to the total rate constant, the reaction is listed in the table under the form (20) rather than in two separate forms. In some instances, a reaction is balanced, but the alkyl radicals formed as products are not specified. E.g.:

$$
\mathrm{CH}_{3} \cdot+\left(\mathrm{CH}_{3}\right)_{2} \mathrm{CHCH}\left(\mathrm{CH}_{3}\right) \mathrm{CH}\left(\mathrm{CH}_{3}\right)_{2} \rightarrow \mathrm{CH}_{4}+\left[\mathrm{C}_{3} \mathrm{H}_{17} \cdot\right]
$$

The unspecified octyl radical inserted in square brackets as product in equation (23) represents all primary, secondary and tertiary octyl radicals that could be formed by abstraction of a $\mathrm{H}$ atom from the reactant $2,3,4-T r i-$ methyl-pentane. There are a number of reactions with the products totally unspecified. In such a case, the word "products" appears after the arrow:

$$
\left(\mathrm{CH}_{3}\right)_{2} \mathrm{C}=\mathrm{CHCH}_{3}+\mathrm{N} \rightarrow \text { products }
$$

\section{Display of Chemical Reactions and Formulae}

A chemical reaction equation should show as clearly as possible the formation of products from the reactants. For that reason, the reactions listed in the table are written on the basis of semi-structural formulas.

\section{Straight chain hydrocarbons. All saturated normal hydrocarbons up to,} and including n-pentane, are written so as to show separately each methyl and methylene group in the chain: $\mathrm{CH}_{4}, \mathrm{CH}_{3} \mathrm{CH}_{3}, \mathrm{CH}_{3} \mathrm{CH}_{2} \mathrm{CH}_{3}, \mathrm{CH}_{3} \mathrm{CH}_{2} \mathrm{CH}_{2} \mathrm{CH}_{3}$, $\mathrm{CH}_{3} \mathrm{CH}_{2} \mathrm{CH}_{2} \mathrm{CH}_{2} \mathrm{CH}_{3}$.

The higher hydrocarbons, from $n$-hexane to $n$-decane, are written in a more condensed form to facilitate the counting of the number of methylene groups in the chain: $\mathrm{CH}_{3}\left(\mathrm{CH}_{2}\right)_{4} \mathrm{CH}_{3}, \mathrm{CH}_{3}\left(\mathrm{CH}_{2}\right)_{5} \mathrm{CH}_{3}, \mathrm{CH}_{3}\left(\mathrm{CH}_{2}\right)_{6} \mathrm{CH}_{3}, \mathrm{CH}_{3}\left(\mathrm{CH}_{2}\right)_{7} \mathrm{CH}_{3}$, $\mathrm{CH}_{3}\left(\mathrm{CH}_{2}\right)_{8} \mathrm{CH}_{3}$. 
The unsaturated hydrocarbons are written so as to show the position of each double or triple bond in the molecule. E.g.:

Ethyne (Acetylene)

$\mathrm{CH} \equiv \mathrm{CH}$

1,2-Propadiene (Allene)

$\mathrm{CH}_{2}=\mathrm{C}=\mathrm{CH}_{2}$

1,3-Butadiyne

$\mathrm{CH} \equiv \mathrm{CC} \equiv \mathrm{CH}$

cis-2-Pentene

c is $-\mathrm{CH}_{3} \mathrm{CH}_{2} \mathrm{CH}=\mathrm{CHCH}_{3}$

1-Heptene

$\mathrm{CH}_{3}\left(\mathrm{CH}_{2}\right)_{4} \mathrm{CH}=\mathrm{CH}_{2}$

Alkyl radicals. The unpaired electron of each alkyl radical is always indicated. E.g.:

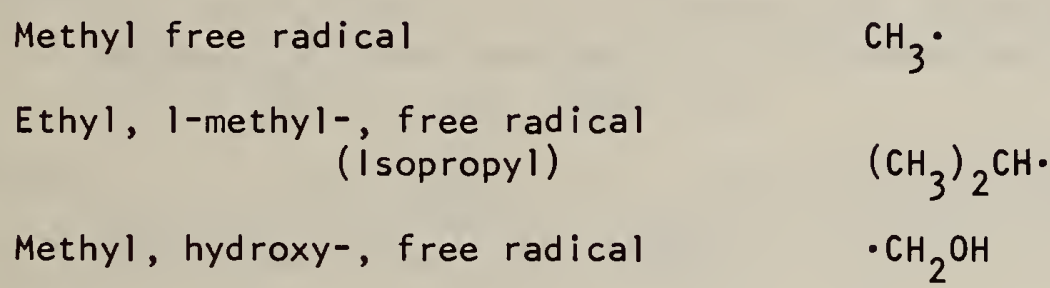

If the unpaired electron of an alkyl radical belongs to a carbon in the middle of the chain, it is indicated inside a parenthesis following the carbon atom. E.g.:

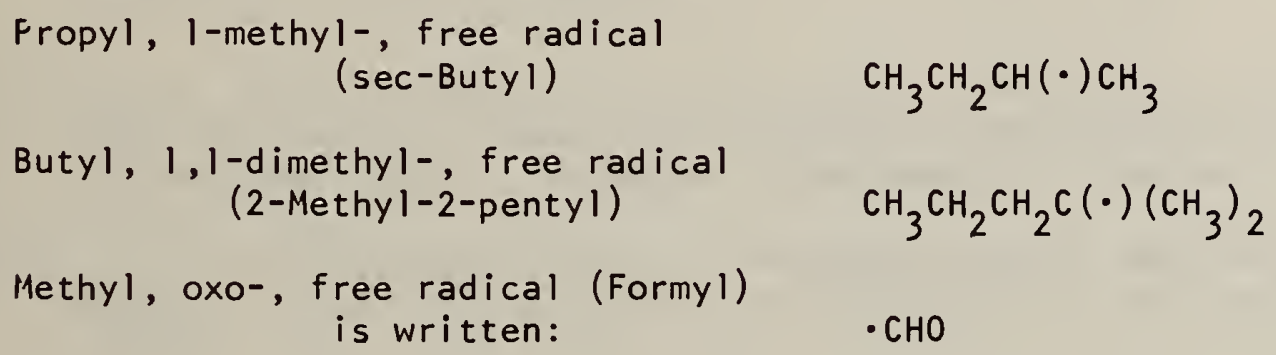

oxy free radicals. If the oxygen atom of an oxy radical is attached to the terminal carbon atom, the radical is written in the usual manner: $\mathrm{CH}_{3} \mathrm{O}$. If the oxygen atom of the oxy radical is attached to a $C$ atom in the middle of the chain, then the oxygen atom, together with the unpaired electron, are inside a parenthesis following the $\mathrm{C}$ atom: $\left(\mathrm{CH}_{3}\right)_{2} \mathrm{C}\left(\mathrm{O}^{\circ}\right) \mathrm{CH}_{2} \mathrm{CH}_{3}$. 
Peroxo, and other free radicals. The rules for writing peroxo, and other free radicals are the same as for the oxy free radicals: $\mathrm{CH}_{3} \mathrm{O}_{2} \cdot \mathrm{CH}_{3} \mathrm{~S}$.. Atoms, like $0, \mathrm{H}, \mathrm{S}, \mathrm{N}$, and simple radicals like $\mathrm{OH}, \mathrm{SH}, \mathrm{NH}, \mathrm{CH}$, $\mathrm{CH}_{2}$, are written without dot. Hydroperoxyl free radical is written $\mathrm{HO}_{2}$. (with dot). 
Baulch, D. L., Drysdale, D. D., Horne, D. G., and Lloyd, A. C., "Evaluated Kinetics Data for High Temperature Reactions, Vol. 1: Homogeneous Gas Phase Reactions of the $\mathrm{H}_{2}-\mathrm{O}_{2}$ System," (Butterworths, London, 1972).

Baulch, D. L., Drysdale, D. D., and Horne, D. G., "Evaluated Kinetic Data for High Temperature Reactions, Vol. 2: Homogeneous Gas Phase Reactions of the $\mathrm{H}_{2}-\mathrm{N}_{2}-\mathrm{O}_{2}$ System," (Butterworths, London, 1973).

Baulch, D. L., Drysdale, D. D., Duxbury, J., and Grant, S. J., "Evaluated Kinetic Data for High Temperature Reactions, Vol. 3: Homogeneous Gas Phase Reactions of the $\mathrm{O}_{2}-\mathrm{O}_{3}$ System, the $\mathrm{CO}-\mathrm{O}_{2}-\mathrm{H}_{2}$ System, and of Sulphur-Containing Species," 13 (Butterworths, London, 1976).

Benson, S. W., Golden, D. M., Lawrence, R. W., Shaw, R., and Woolfolk, R. W., "Estimating the Kinetics of Combustion Including Reactions Involving Oxides of Nitrogen and Sulfur," Environmental Protection Agency Report No. EPA-600/2-75-019, August 1975.

Benson, S. W., and O'Neal, H. E., "Kinetic Data on Gas Phase Unimolecular Reactions," NBS-NSRDS-21 (1970). (Supt. Doc., U.S. Govt. Printing office, Washington, D.C. 20402).

Engleman, V. S., "Survey and Evaluation of Kinetic Data on Reactions in Methane/Air Combustion," Environmental Protection Agency Report No. EPA-600/2-76-003, January 1976.

Herron, J. T., and Huie, R. E., "Rate Constants for the Reactions of Atomic Oxygen $\left(0^{3} \mathrm{P}\right)$ with Organic Compounds in the Gas Phase," J. Phys. Chem. Ref. Data 2 , 467-518 (1973).

Kerr, J. A., and Parsonage, M. J., "Evaluated Kinetic Data on Gas Phase Addition Reactions: Reactions of Atoms and Radicals with Alkenes, Alkynes and Aromatic Compounds," (Butterworths, London, 1972).

Kerr, J. A., and Parsonage, M. J., "Evaluated Kinetic Data on Gas Phase Hydrogen Transfer Reactions of Methyl Radicals," (Butterworths, London 1976).

Kondratiev, V. N., "Konstanty Skorosti Gazofaznykh Reaktsij Spravochnik," (Izdatelstvo "Nauka", Moskva, 1970); also issued as "Rate Constants of Gas Phase Reactions, Reference Book," R. M. Fristrom, Ed., Translated by L. J. Holtschlag COM-72-10014, Office of Standard Reference Data, NBS (1972) (Distributed by National Technical Information Service, Springfield, Virginia 22151).

Lloyd, A. C., "Evaluated and Estimated Kinetic Data for Gas Phase Reactions of the Hydroperoxyl Radical," Int. J. Chem. Kinet. $\underline{6}$, 169-228 (1974). 


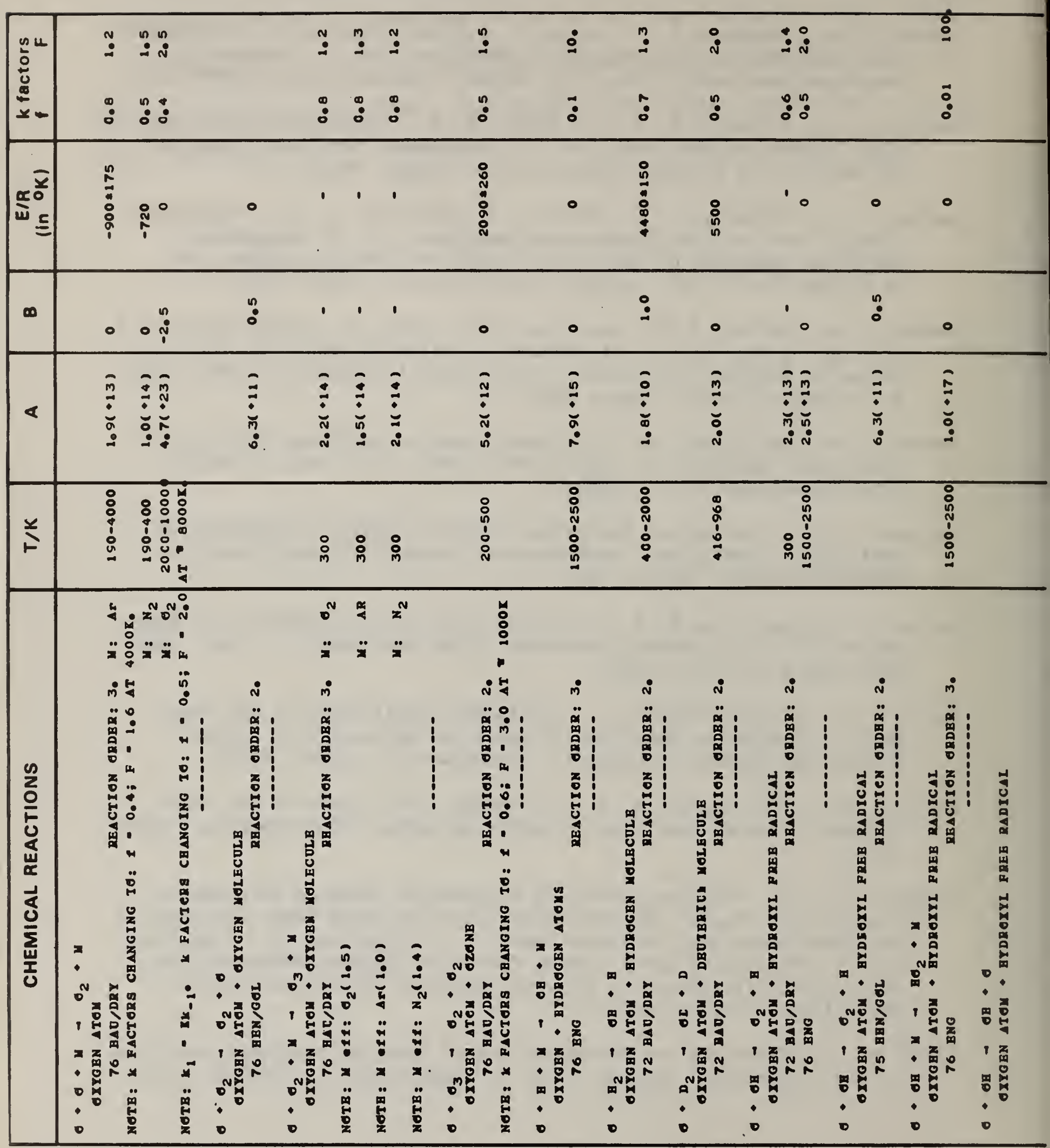




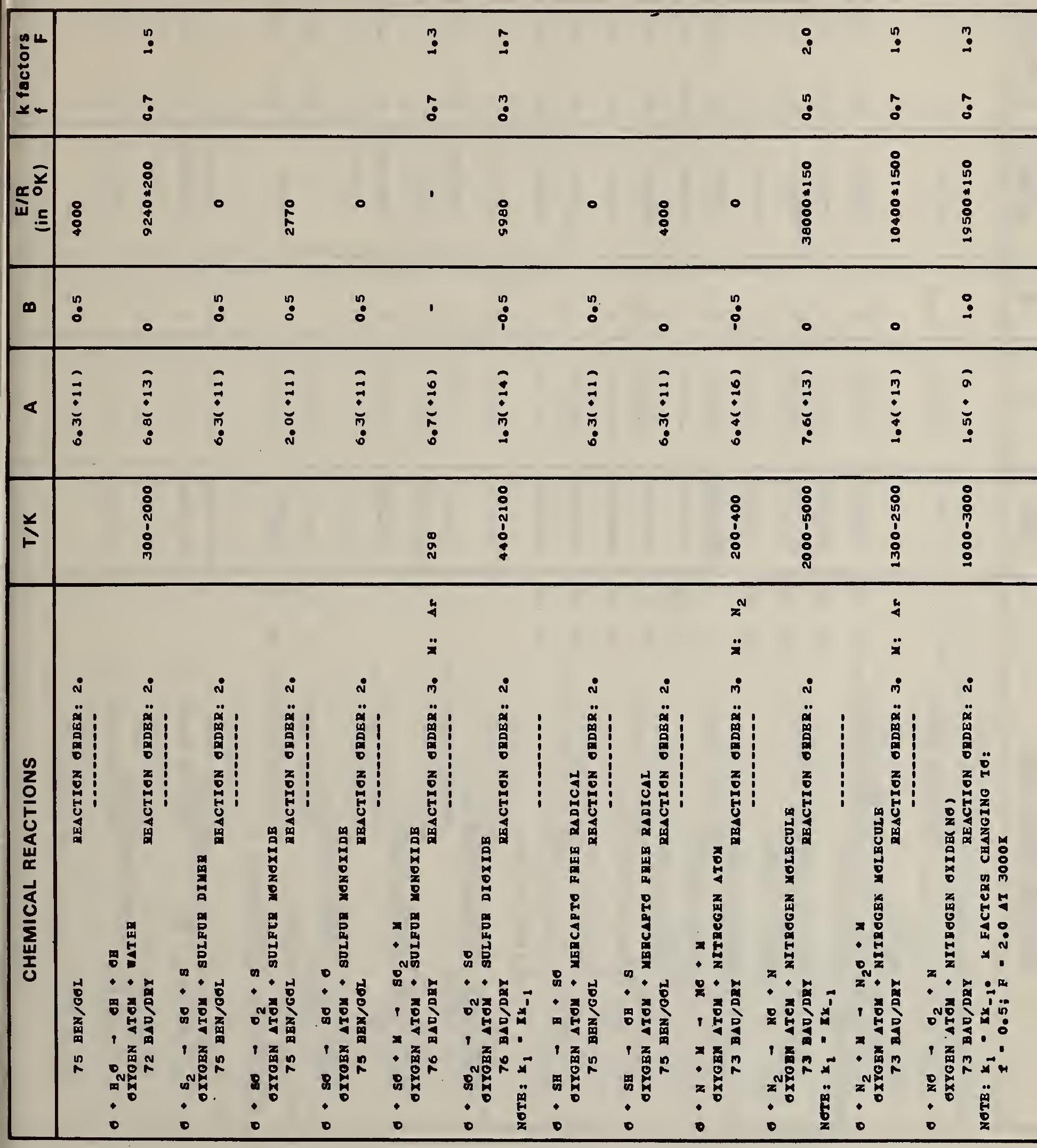




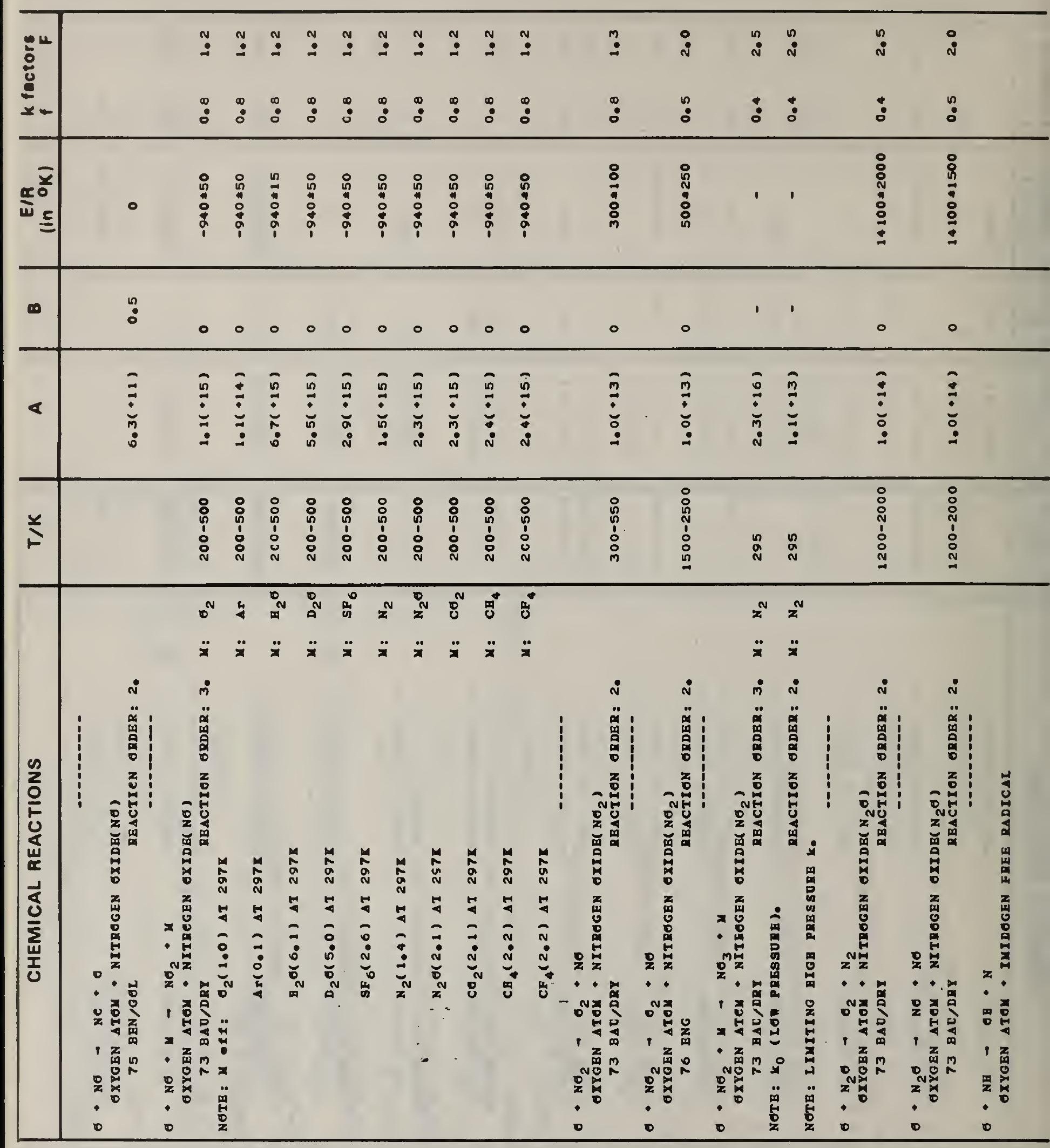




\begin{tabular}{|c|c|c|c|c|c|c|c|c|c|c|c|c|}
\hline 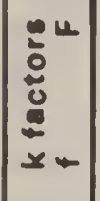 & $\begin{array}{l}\stackrel{0}{\sim} \\
\stackrel{0}{0}\end{array}$ & & & $\begin{array}{l}\stackrel{N}{m} \\
\dot{m} \\
\stackrel{m}{0}\end{array}$ & $\begin{array}{l}\stackrel{N}{m} \\
\stackrel{n}{0} \\
\stackrel{m}{0}\end{array}$ & $\begin{array}{l}\stackrel{N}{\oplus} \\
\stackrel{0}{0} \\
\stackrel{m}{\circ}\end{array}$ & $\begin{array}{l}\stackrel{0}{\text { i }} \\
\stackrel{0}{\circ}\end{array}$ & $\begin{array}{l}\stackrel{m}{:}: 0 \\
: \stackrel{0}{\circ}\end{array}$ & & & $\stackrel{N}{:}$ & $\begin{array}{l}\text { : } \\
:\end{array}$ \\
\hline 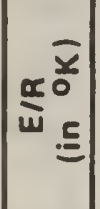 & 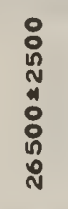 & : & 0 & : & 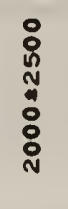 & $\begin{array}{l}\text { : } \\
\stackrel{4}{y} \\
\vdots \\
\stackrel{0}{n} \\
\underline{n}\end{array}$ & 。 & 㽞 & $\begin{array}{l}\stackrel{0}{0} \\
\stackrel{\leftrightarrow}{0} \\
0\end{array}$ & 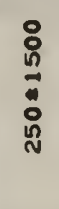 & $\begin{array}{l}: \\
: \\
: \\
: \\
\stackrel{\circ}{n} \\
\end{array}$ & ' \\
\hline$\infty$ & $\circ$ & $\stackrel{?}{0}$ & $\stackrel{?}{0}$ & $\stackrel{n}{0}$ & $\stackrel{n}{0}$ & $\hat{\circ}$ & - & 00 & ○ & $\stackrel{\circ}{9}$ & $\stackrel{8}{\square}$ & . \\
\hline$\varangle$ & $\begin{array}{l}\hat{n} \\
\vdots \\
\vdots\end{array}$ & $\bar{\vdots}$ & $\bar{\Xi}$ & $\begin{array}{l}0 \\
\vdots \\
\vdots\end{array}$ & $\begin{array}{l}\vdots \\
\vdots \\
\vdots\end{array}$ & $\begin{array}{l}\vdots \\
\vdots \\
\vdots\end{array}$ & $\begin{array}{l}\hat{m} \\
\vdots \\
\dot{0} \\
\dot{n}\end{array}$ & 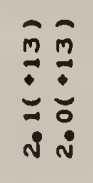 & $\begin{array}{l}\vdots \\
\vdots \\
\dot{N} \\
\dot{m}\end{array}$ & $\bar{\Xi}$ & $\bar{\Xi}$ & : \\
\hline$\stackrel{x}{F}$ & $\begin{array}{l}0 \\
\vdots \\
0 \\
\vdots \\
\vdots \\
0\end{array}$ & & & 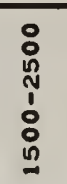 & $\begin{array}{l}0 \\
: \\
\mathscr{n} \\
\vdots \\
\vdots \\
\vdots \\
0\end{array}$ & 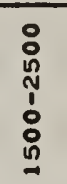 & 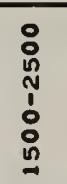 & 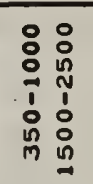 & 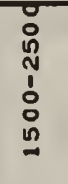 & 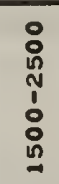 & $\begin{array}{l}\circ \\
\stackrel{0}{0} \\
N \\
1 \\
\vdots \\
: \\
0\end{array}$ & : \\
\hline
\end{tabular}

:

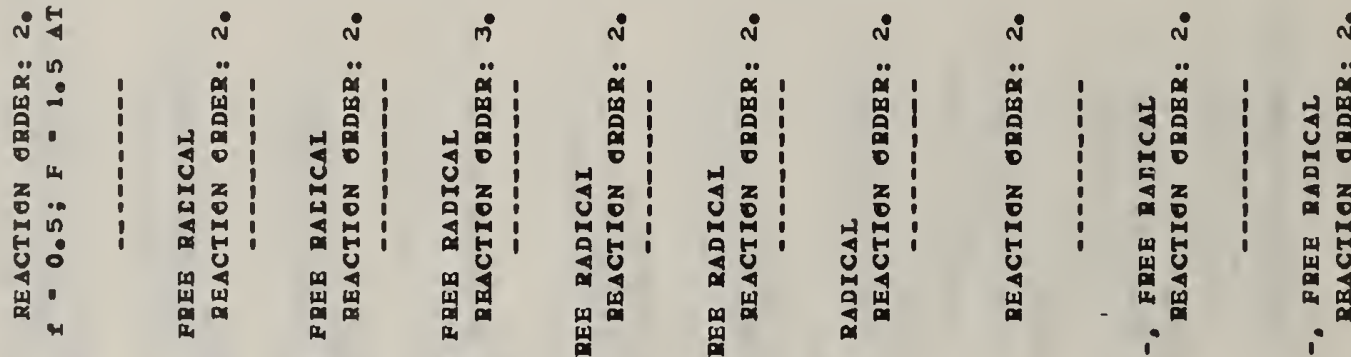

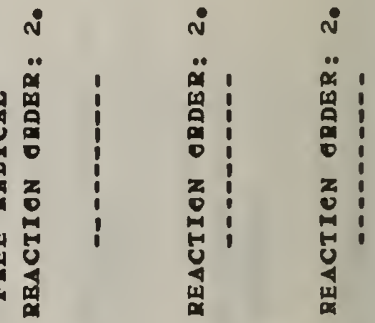

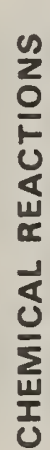

(1)

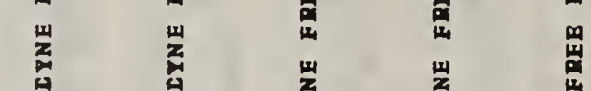

:

要要

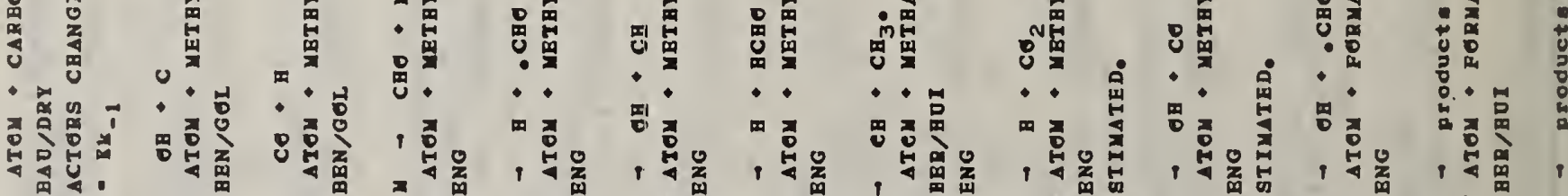

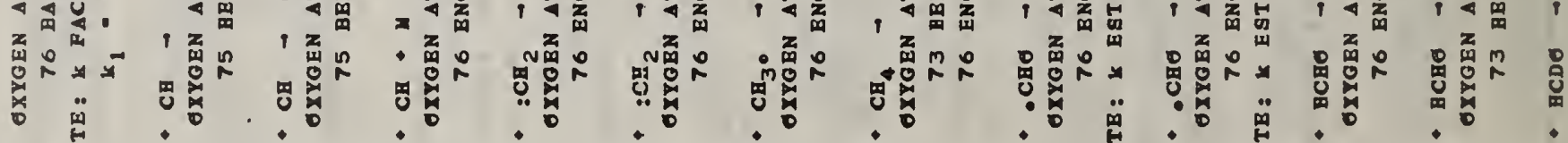




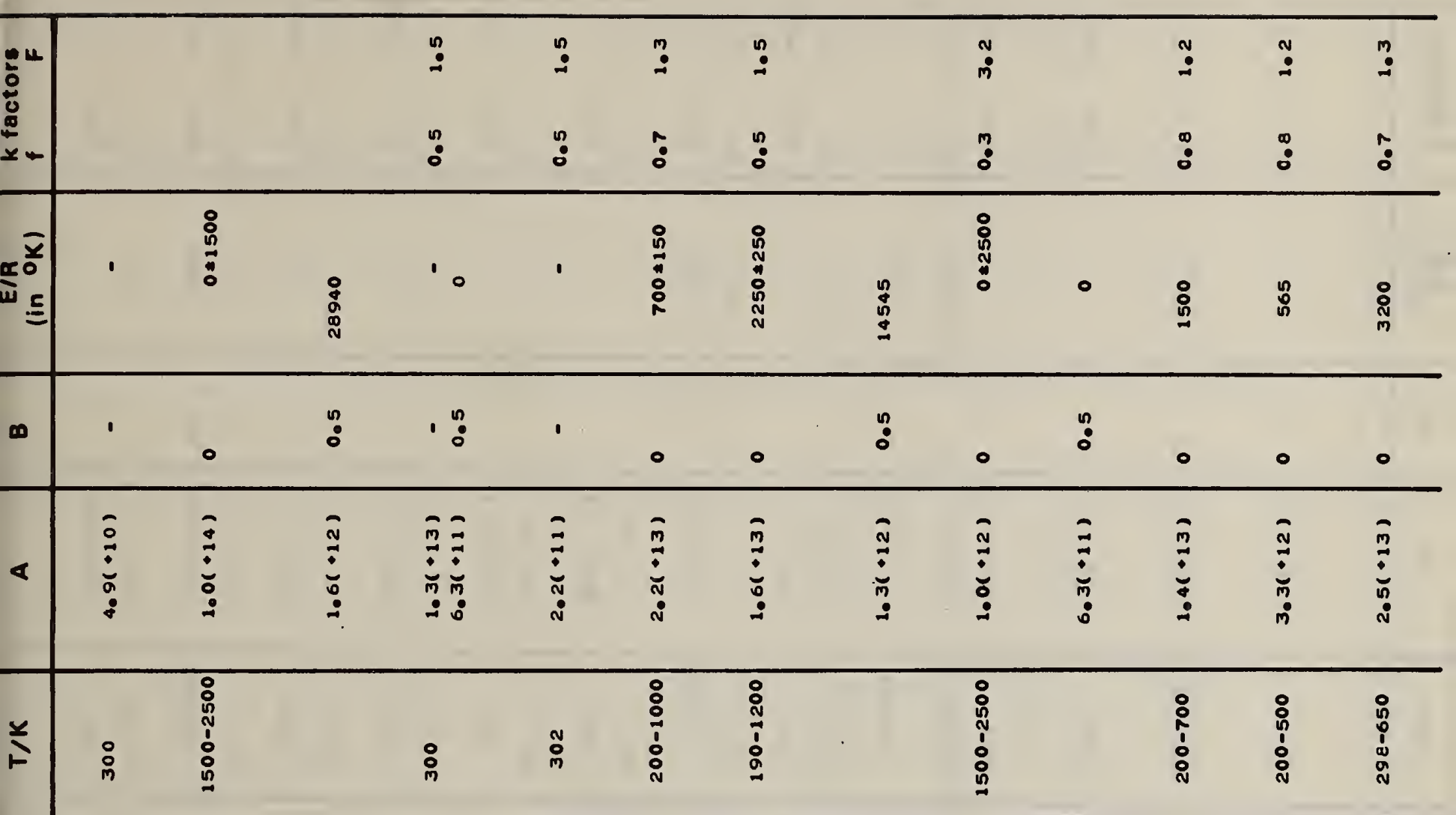




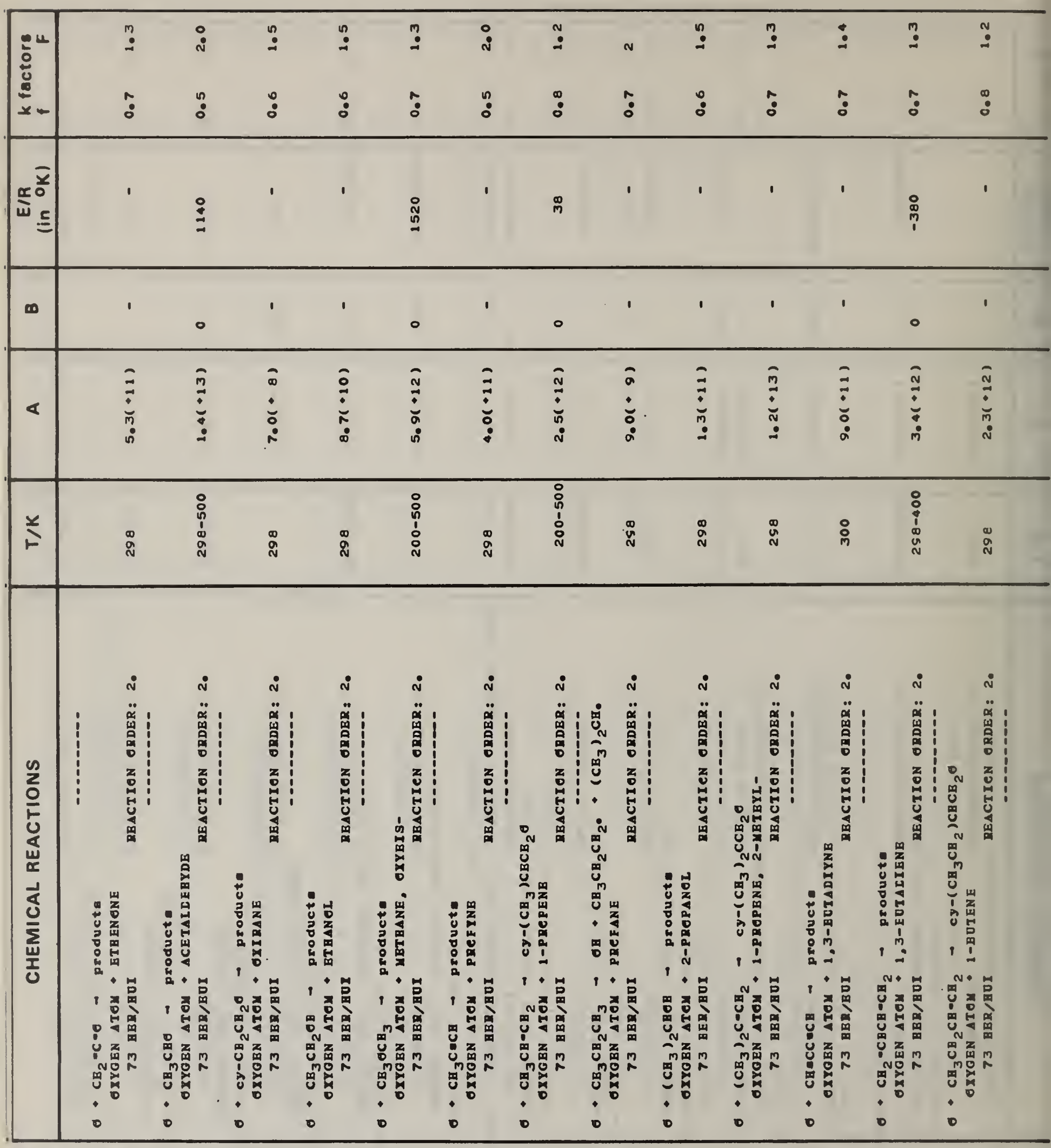




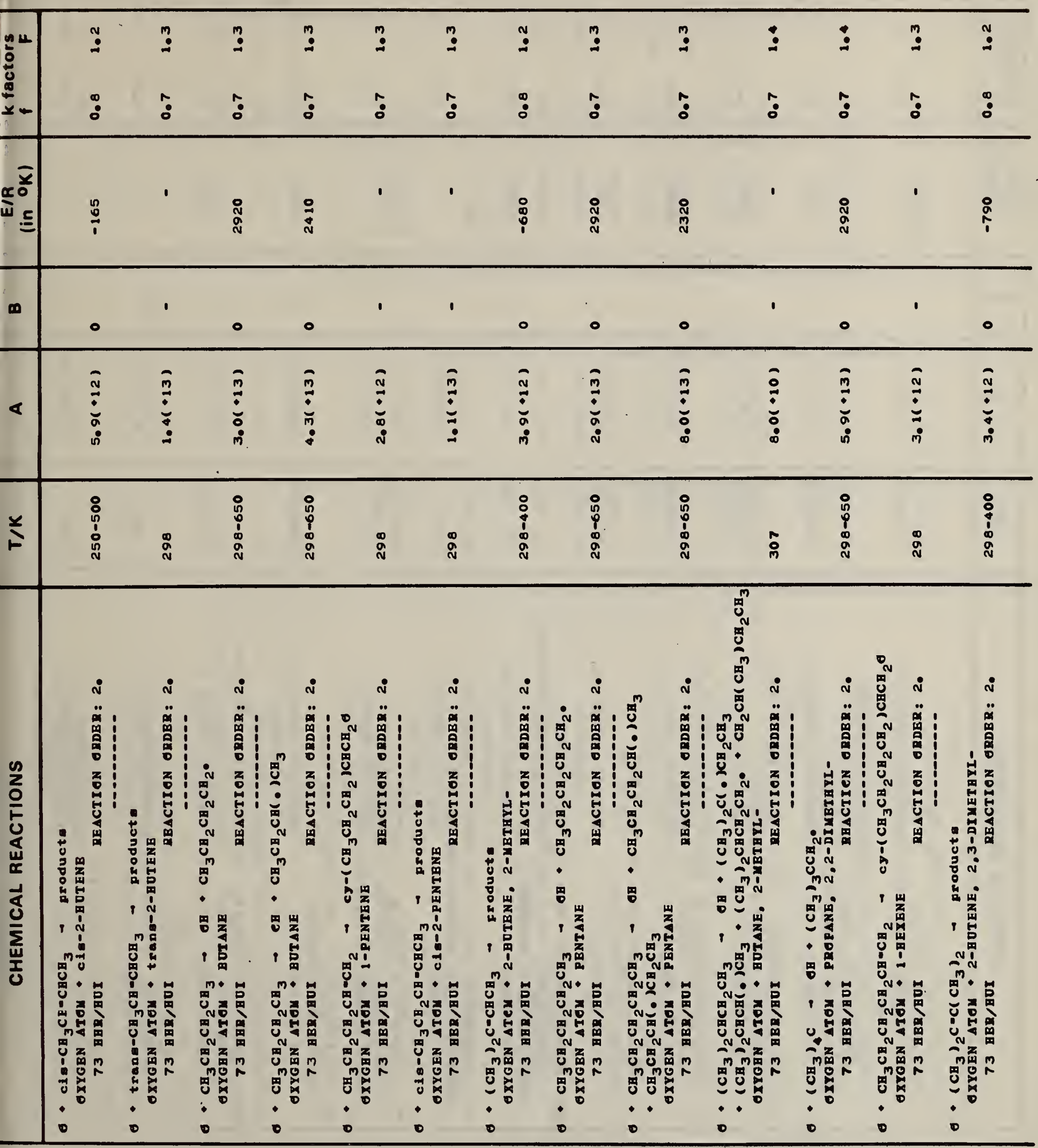




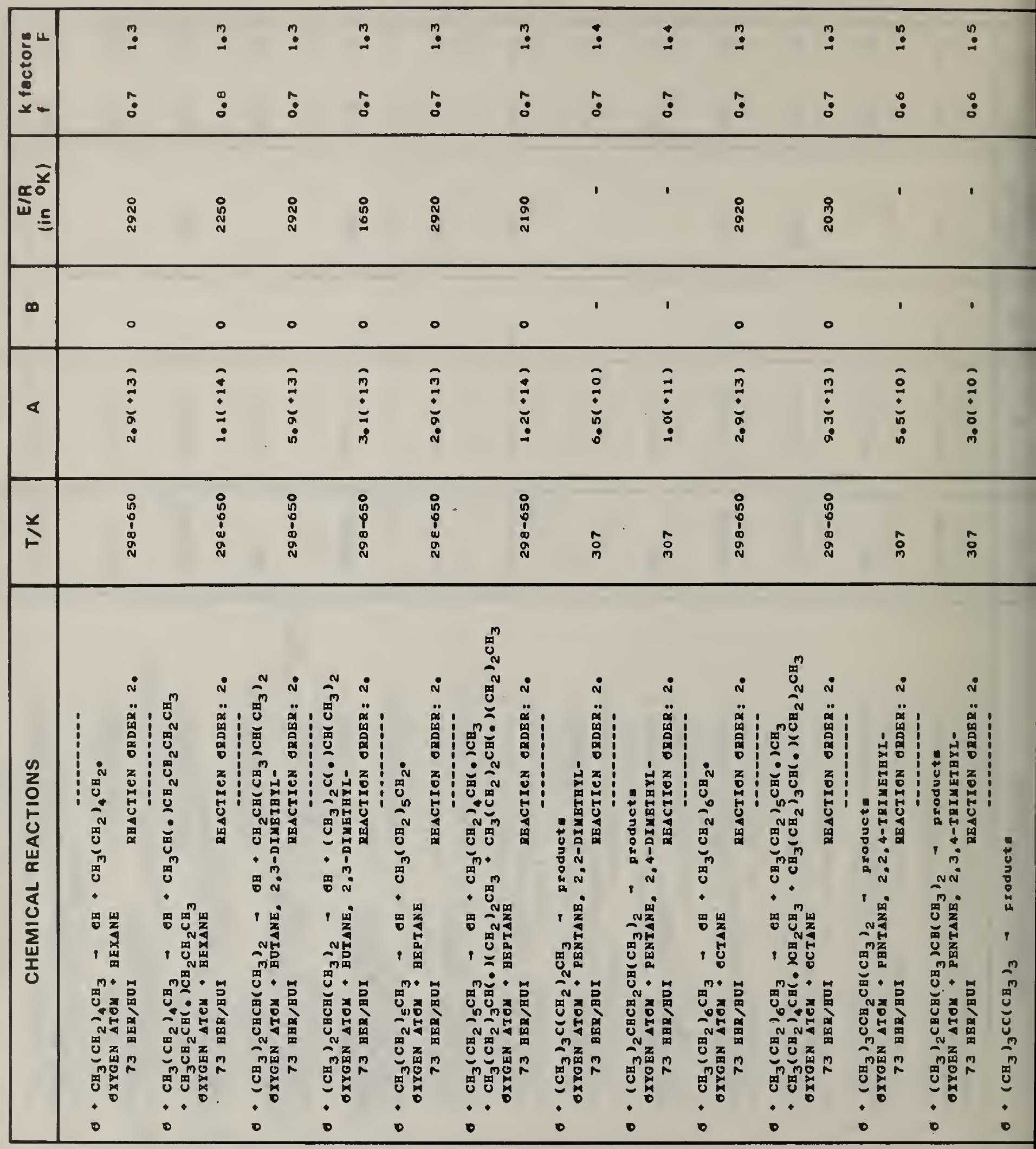




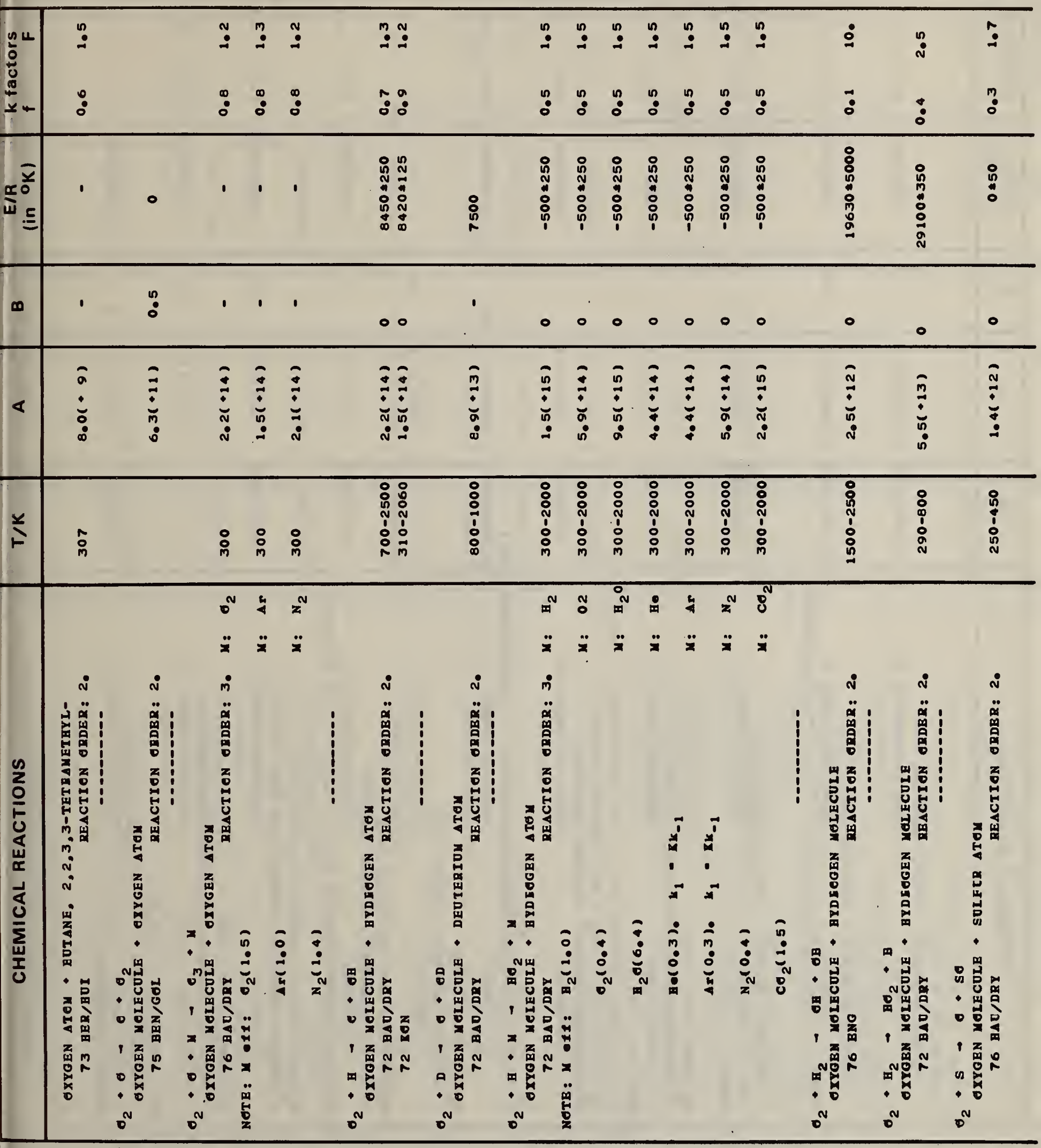




\begin{tabular}{|c|c|c|c|c|c|c|c|c|c|c|c|c|c|c|c|c|}
\hline 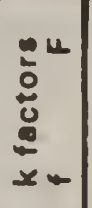 & $\begin{array}{l}: \\
:\end{array}$ & $\begin{array}{l}\text { ? } \\
\text { : }\end{array}$ & & $\begin{array}{l}\stackrel{n}{N} \\
\dot{0}\end{array}$ & $\begin{array}{l}\text { ? } \\
:\end{array}$ & $\begin{array}{l}\stackrel{5}{:} \\
:\end{array}$ & $\stackrel{?}{:}$ & $\begin{array}{l}\stackrel{n}{0} \\
:\end{array}$ & & & $\begin{array}{l}: \\
: \\
:\end{array}$ & $\begin{array}{l}\stackrel{N}{m} \\
\stackrel{m}{:} \\
\stackrel{m}{0}\end{array}$ & $\begin{array}{l}\stackrel{n}{\dot{m}} \\
\stackrel{m}{0}\end{array}$ & & & 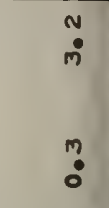 \\
\hline $\begin{array}{l}\underline{x} \overline{0} \\
w \\
w\end{array}$ & $\begin{array}{l}\text { o } \\
0 \\
0 \\
0 \\
0 \\
0 \\
m\end{array}$ & $\begin{array}{l}\stackrel{0}{0} \\
\vdots \\
\dot{0} \\
m\end{array}$ & & $\begin{array}{l}: \\
\vdots \\
\vdots \\
\vdots \\
0 \\
0 \\
0\end{array}$ & & 䯧 & ঃ & $\begin{array}{l}0 \\
: \\
\emptyset \\
\vdots \\
\vdots \\
i\end{array}$ & 0 & & $\begin{array}{l}\text { : } \\
\text { N } \\
\vdots \\
\vdots \\
\vdots \\
\vdots \\
\text { N }\end{array}$ & 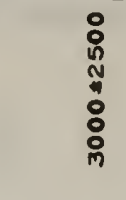 & 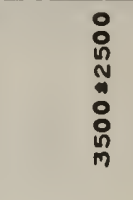 & $\vdots$ & & $:$ \\
\hline$\infty$ & ○ & $\stackrel{9}{9}$ & & ○ & & ० & 0 & 0 & $\therefore$ & & ० & $\stackrel{n}{0}$ & $\stackrel{n}{\circ}$ & 。 & & 0 \\
\hline$\varangle$ & $\begin{array}{l}\vdots \\
\vdots \\
\vdots \\
\vdots\end{array}$ & å & & $\stackrel{n}{\vdots}$ & & $\begin{array}{c}\bar{s} \\
\vdots \\
\vdots \\
\vdots\end{array}$ & $\begin{array}{l}\alpha \\
\dot{\omega} \\
\end{array}$ & $\begin{array}{l}\tilde{a} \\
\dot{a} \\
\dot{n}\end{array}$ & $\begin{array}{l}\vdots \\
\vdots \\
\dot{\vdots} \\
\dot{0}\end{array}$ & & 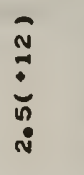 & $\begin{array}{l}\vdots \\
\vdots \\
\vdots \\
\vdots\end{array}$ & $\begin{array}{l}\vdots \\
\vdots \\
\vdots\end{array}$ & & & $\dot{m}$ \\
\hline 兴 & $\begin{array}{l}\stackrel{0}{0} \\
\overline{1} \\
\vdots \\
\vdots\end{array}$ & $\begin{array}{l}\text { : } \\
\vdots \\
\vdots \\
\vdots \\
\vdots\end{array}$ & & $\begin{array}{l}\vdots \\
\vdots \\
\vdots \\
\vdots \\
\vdots \\
\end{array}$ & & 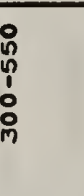 & 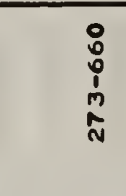 & 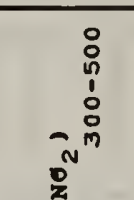 & & & 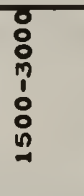 & $\begin{array}{l} \\
\vdots \\
\stackrel{0}{2} \\
\vdots \\
\vdots \\
\vdots\end{array}$ & & & & : \\
\hline 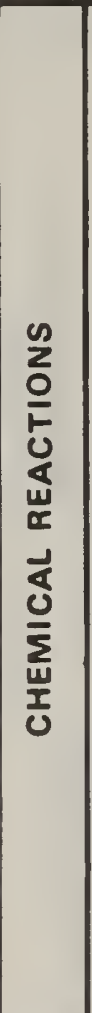 & 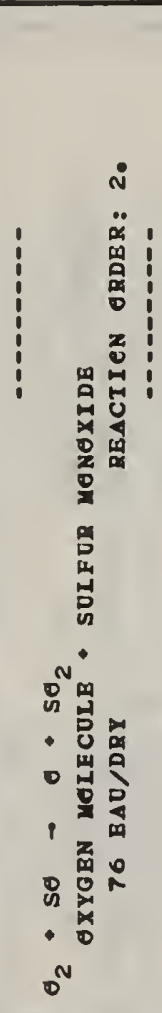 & 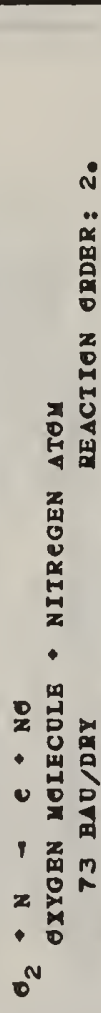 & 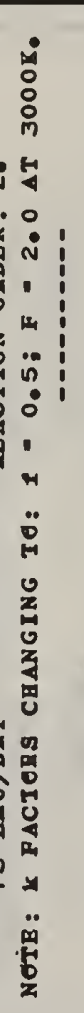 & 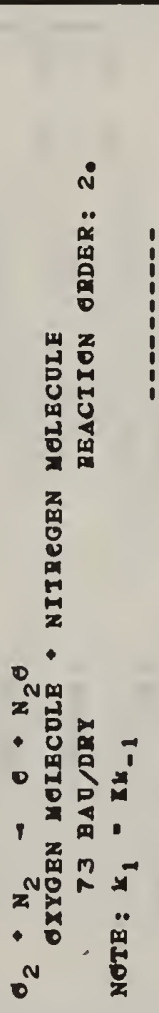 & 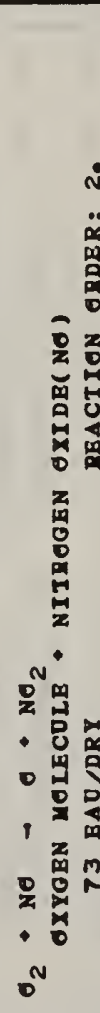 & 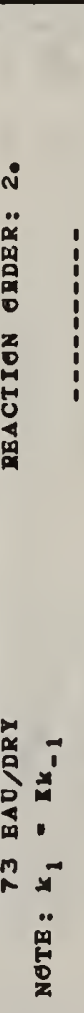 & 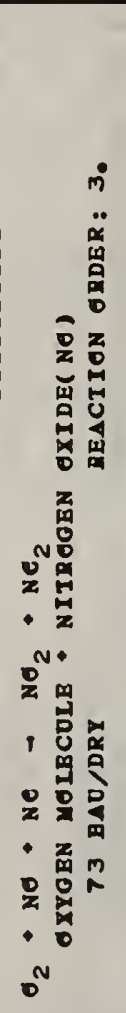 & 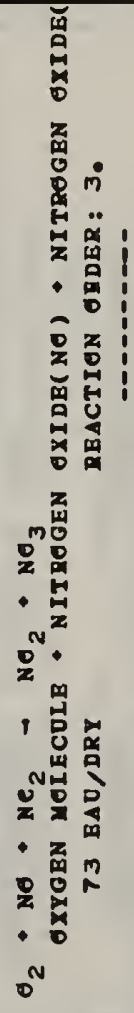 & 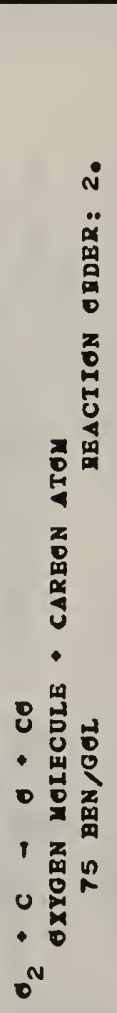 & 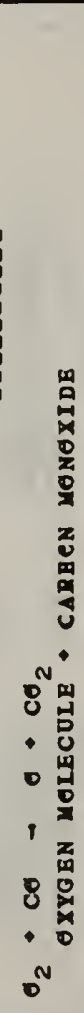 & 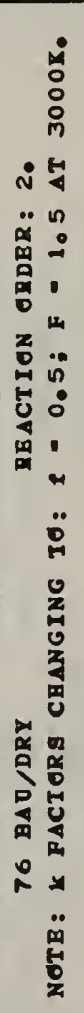 & 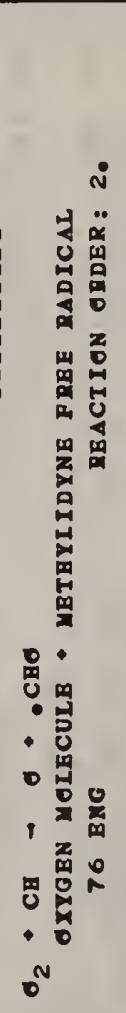 & 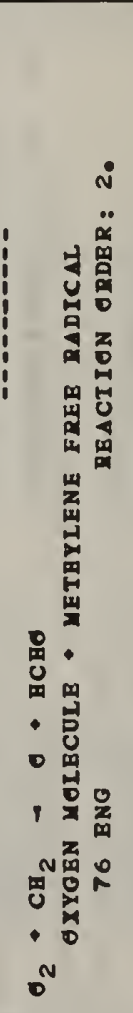 & 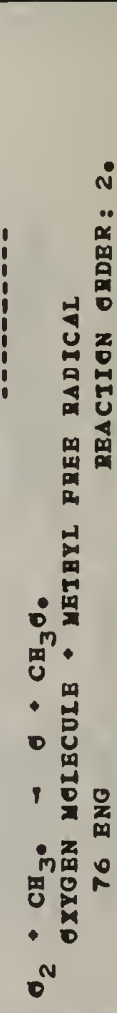 & 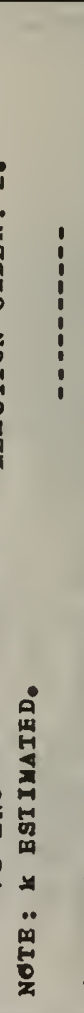 & 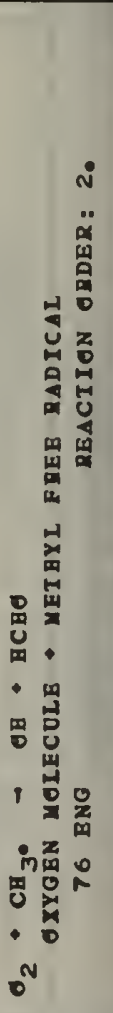 \\
\hline
\end{tabular}




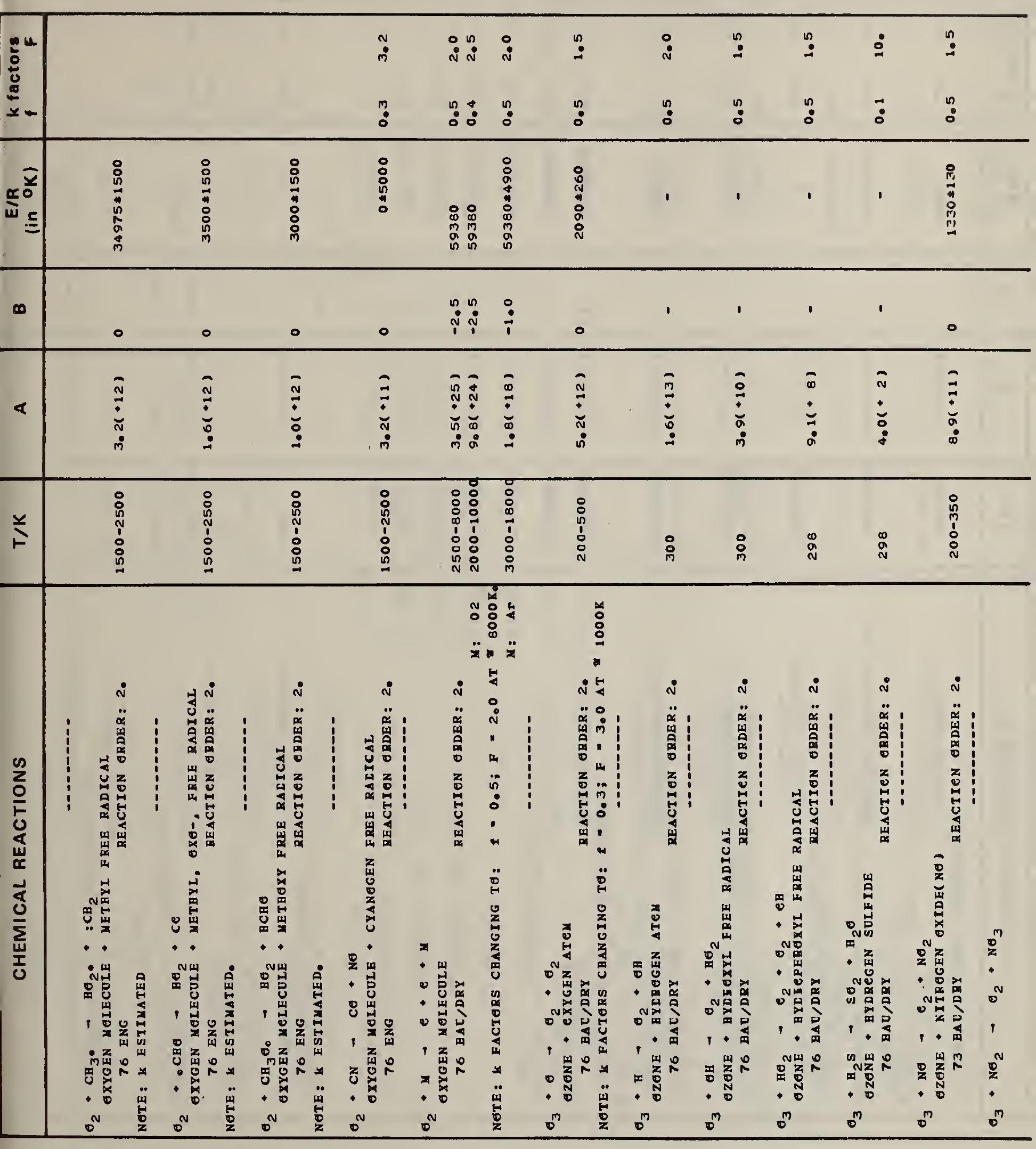




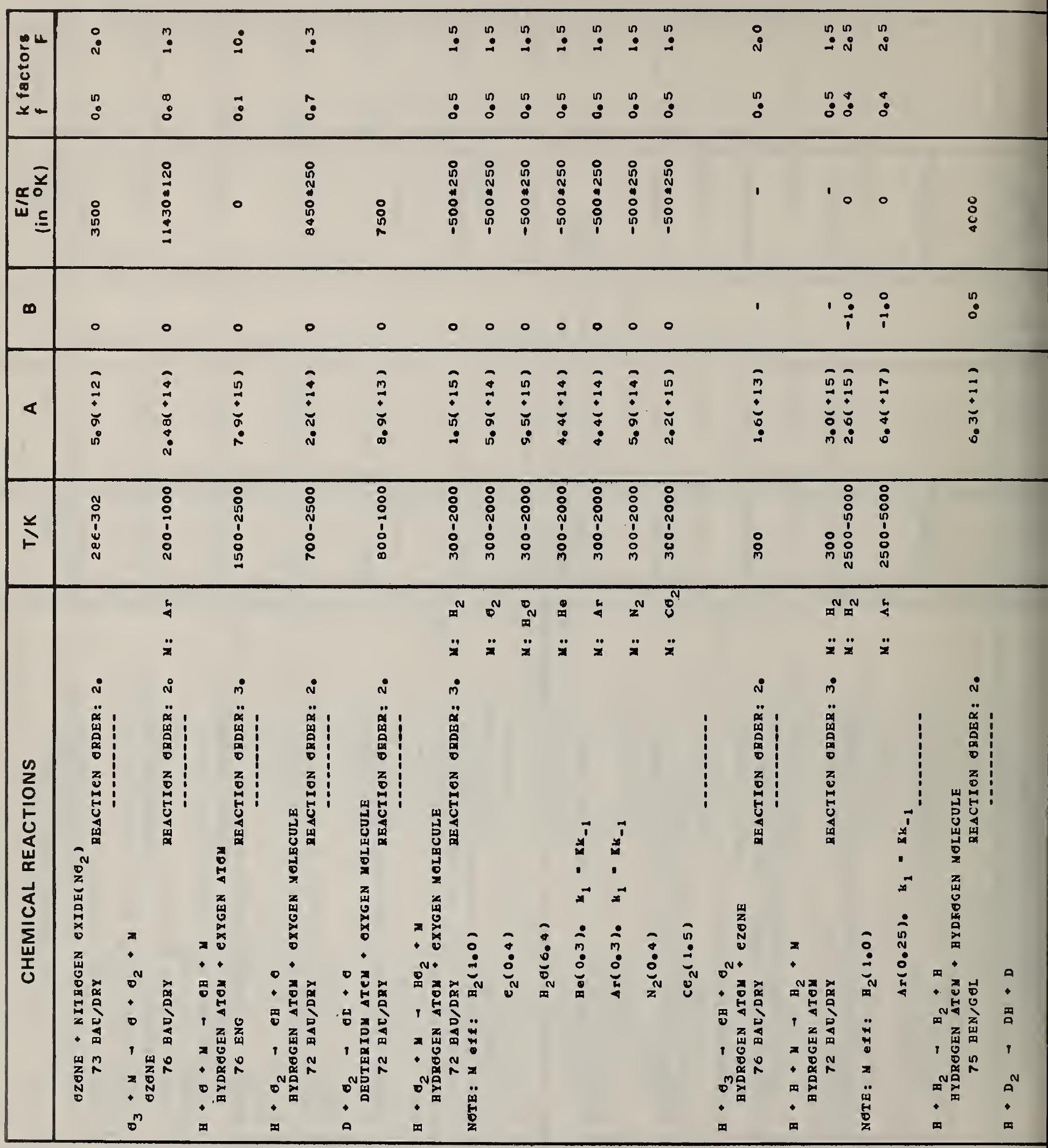




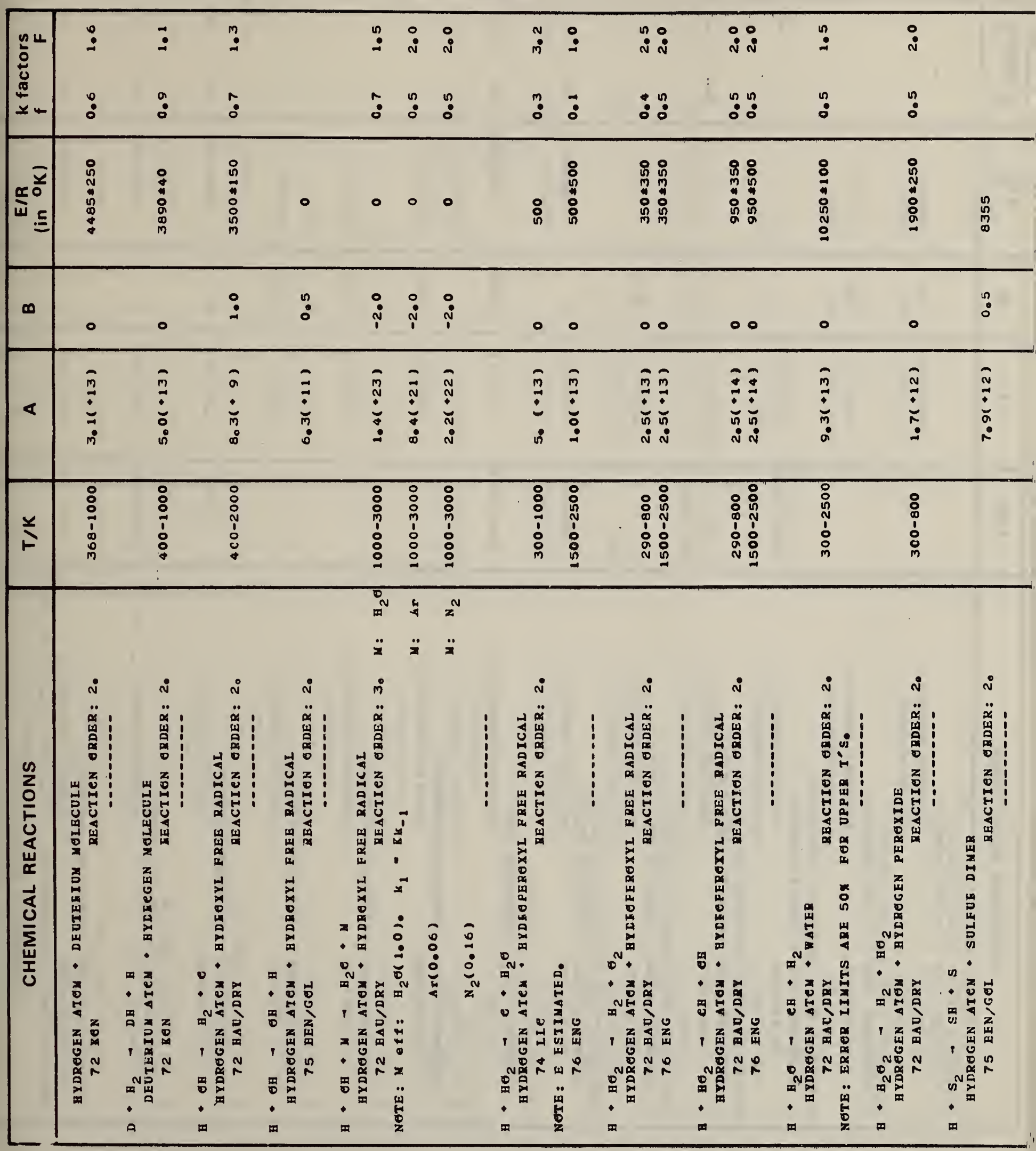




\begin{tabular}{|c|c|c|c|c|c|c|c|c|c|c|c|c|c|c|}
\hline$\frac{0^{4}}{\vdots}$ & & & $\begin{array}{l}\stackrel{?}{:} \\
\stackrel{0}{0}\end{array}$ & $\begin{array}{l}\stackrel{n}{:} \\
\stackrel{n}{0}\end{array}$ & & $\begin{array}{l}? \\
: \\
?\end{array}$ & $\begin{array}{l}\dot{0} \\
\ddot{0}\end{array}$ & & & & $\begin{array}{l}\stackrel{n}{:} \\
:\end{array}$ & $\begin{array}{l}\stackrel{0}{\text { i }} \\
\stackrel{4}{0}\end{array}$ & $\begin{array}{l}\text { ? } \\
\text { : }\end{array}$ & 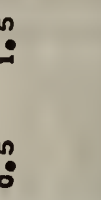 \\
\hline 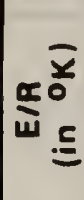 & 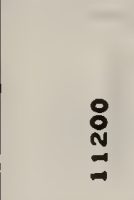 & 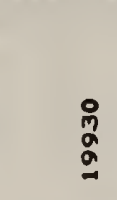 & ' & ' & 0 & $\begin{array}{l}0 \\
\vdots \\
\vdots \\
\vdots \\
\infty\end{array}$ & $\frac{8}{0}$ & 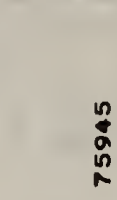 & : & 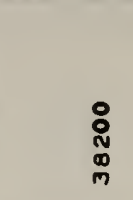 & $\begin{array}{l}\stackrel{8}{1} \\
\vdots \\
\grave{0} \\
1\end{array}$ & 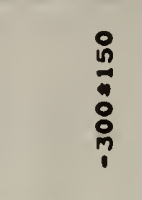 & $\begin{array}{l}: \\
\vdots \\
\vdots \\
\vdots \\
\vdots \\
\vdots\end{array}$ & $\begin{array}{l}: \\
: \\
b_{1}^{\circ} \\
\vdots \\
\vdots\end{array}$ \\
\hline$\infty$ & $\stackrel{n}{0}$ & $\stackrel{n}{0}$ & ' & ' & $\stackrel{n}{0}$ & 0 & $\stackrel{0}{0}$ & $\stackrel{n}{\circ}$ & $\stackrel{n}{:}$ & $\stackrel{?}{:}$ & ○ & ० & & \\
\hline$<$ & $\stackrel{\bar{N}}{\vdots}$ & $\begin{array}{l}\bar{\vdots} \\
\dot{\vdots} \\
\vdots\end{array}$ & $\stackrel{n}{\ddot{n}}$ & 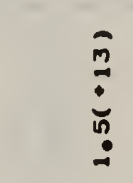 & $\stackrel{\vdots}{\grave{j}}$ & $\begin{array}{l}\hat{N} \\
\dot{\varpi} \\
\stackrel{0}{0}\end{array}$ & $\begin{array}{l}5 \\
\vdots \\
\vdots \\
\end{array}$ & $\begin{array}{l}\hat{m} \\
\vdots \\
\dot{0}\end{array}$ & $\begin{array}{l}\hat{N} \\
\vdots \\
\grave{n} \\
\stackrel{0}{0}\end{array}$ & $\begin{array}{l}\hat{N} \\
\vdots \\
\dot{0}\end{array}$ & : & $\begin{array}{l}\tilde{n} \\
\vdots \\
\vdots\end{array}$ & $\begin{array}{l}\vdots \\
\vdots \\
\end{array}$ & 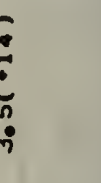 \\
\hline$\stackrel{Y}{F}$ & & & 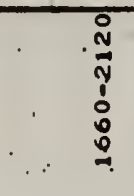 & $\stackrel{\infty}{\infty} \underset{N}{\infty}$ & & $\begin{array}{l}0 \\
\vdots \\
\vdots \\
\vdots \\
\vdots\end{array}$ & 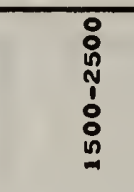 & & & & 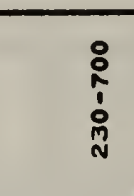 & 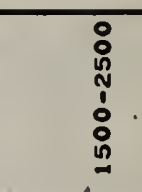 & 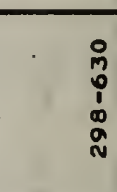 & רo \\
\hline 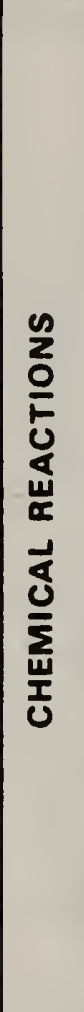 & 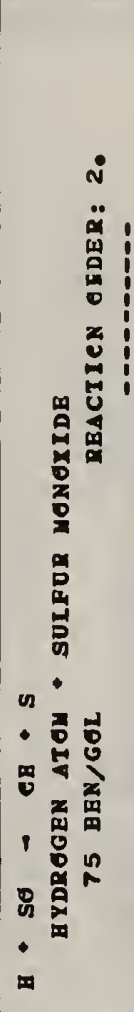 & 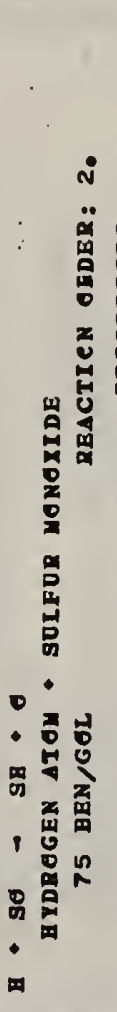 & 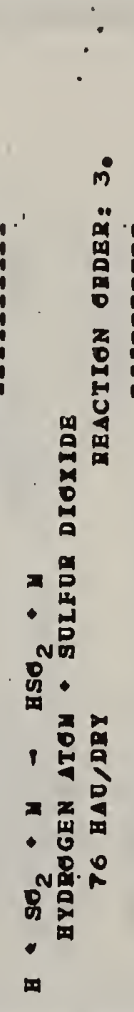 & 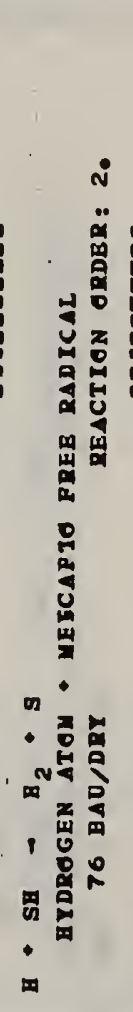 & 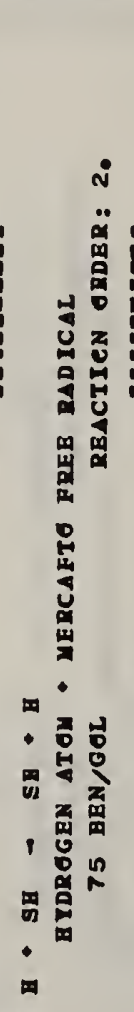 & 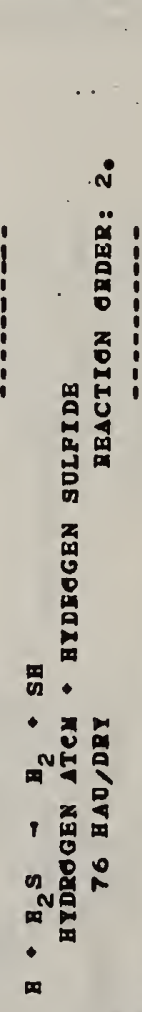 & $\begin{array}{l}r \\
\\
\end{array}$ & 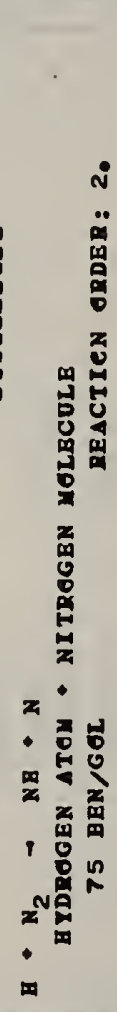 & 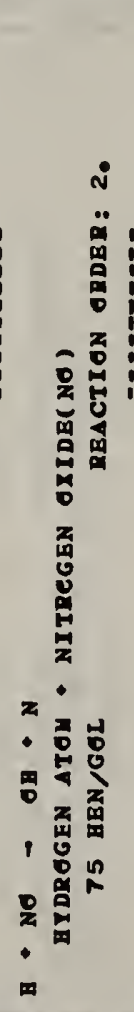 & 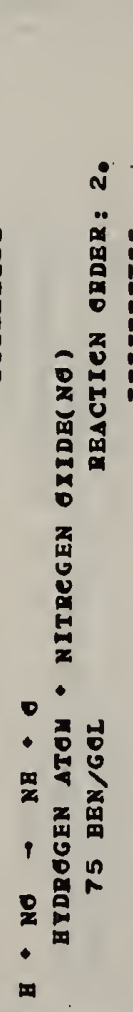 & 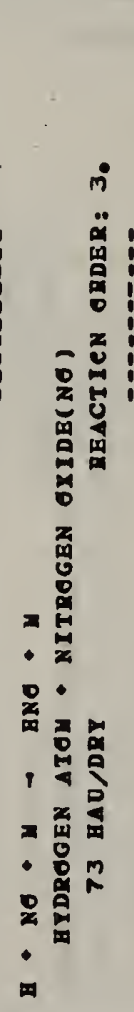 & 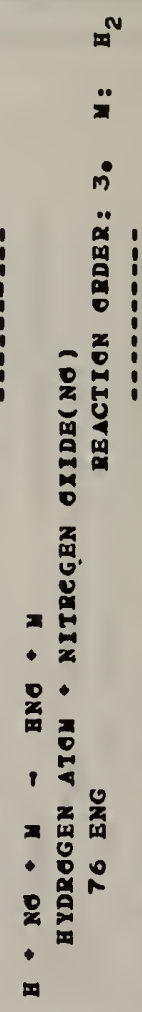 & 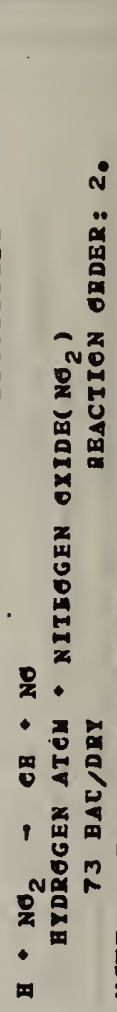 & 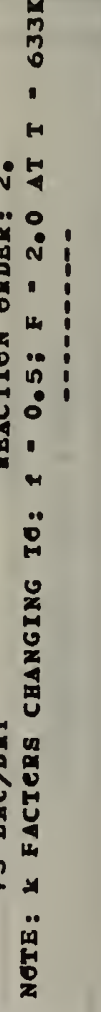 \\
\hline
\end{tabular}




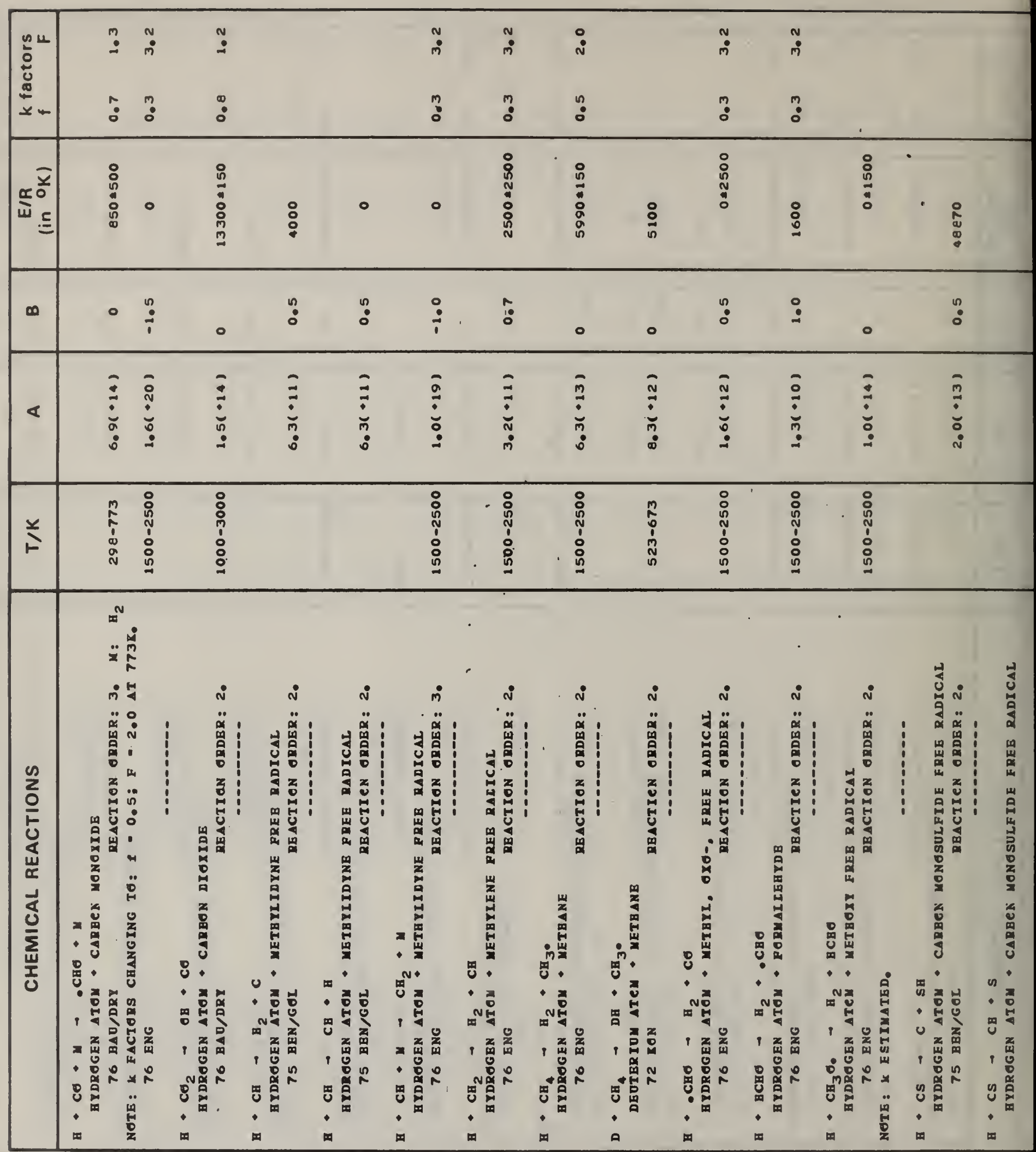




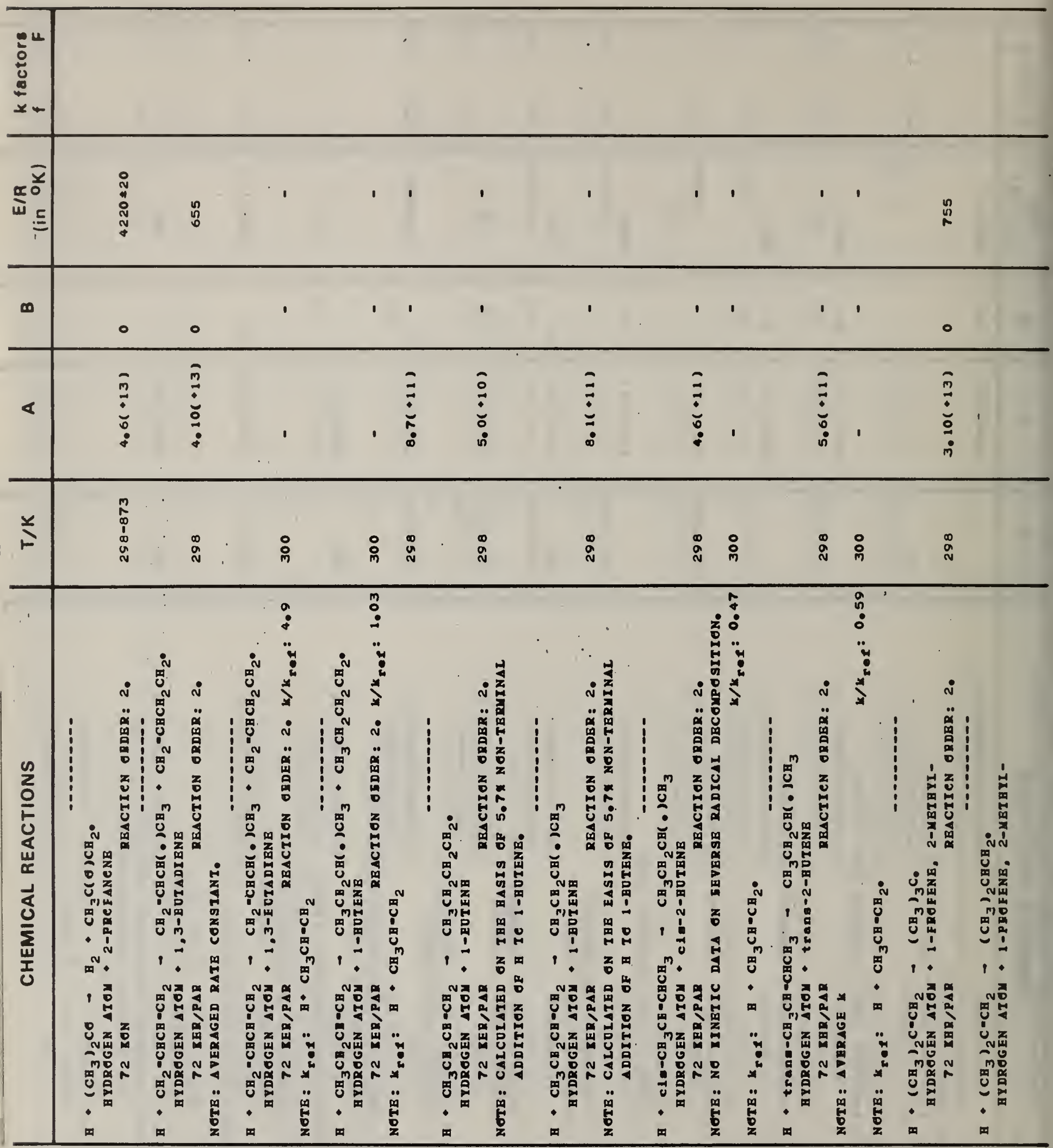




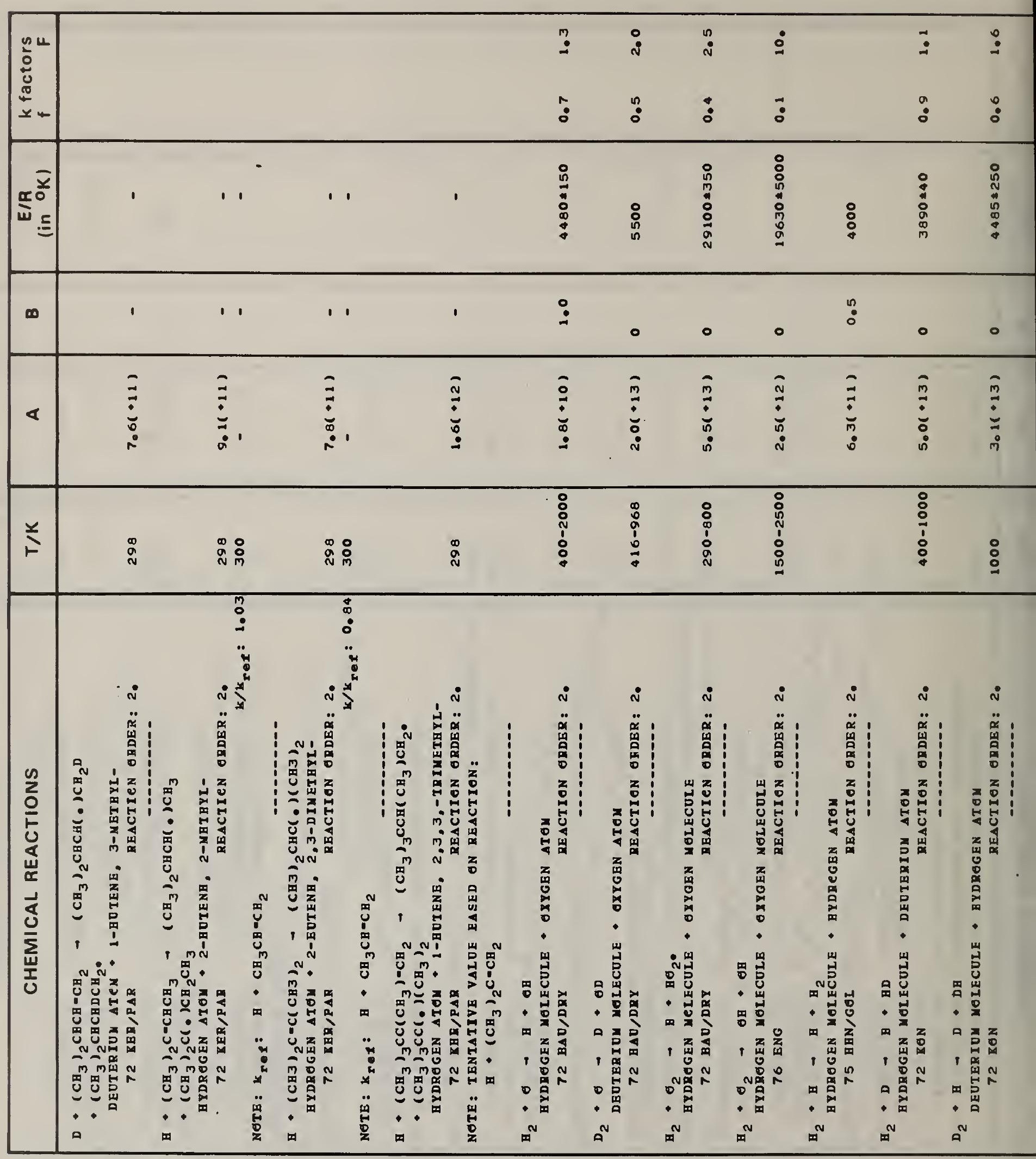




\begin{tabular}{|c|c|c|c|c|c|c|c|c|c|c|c|c|c|}
\hline 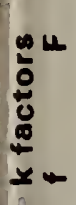 & $\begin{array}{l}N \\
\vdots \\
\infty \\
0\end{array}$ & $\begin{array}{l}: \\
\text { i } \\
\text { ! }\end{array}$ & $\begin{array}{l}: \\
\text { N } \\
:\end{array}$ & $\begin{array}{l}\text { ? } \\
?:\end{array}$ & & 1 & & $\begin{array}{l}N \\
m \\
m \\
m\end{array}$ & & $\begin{array}{l}? \\
:\end{array}$ & $\because$ & $\begin{array}{l}? \\
: \\
?\end{array}$ & $\begin{array}{l}? \\
: \\
:\end{array}$ \\
\hline$\stackrel{0}{\omega}^{0}$ & 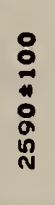 & 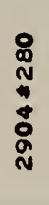 & 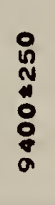 & & $\begin{array}{l}0 \\
0 \\
0 \\
0\end{array}$ & $\underset{\infty}{8}$ & $\begin{array}{l}8 \\
\text { in } \\
-1\end{array}$ & $\begin{array}{l}8 \\
8 \\
\qquad \\
N \\
\qquad \\
0 \\
\qquad \\
n\end{array}$ & 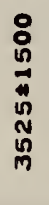 & $\begin{array}{l}8 \\
8 \\
0 \\
0 \\
8 \\
\text { in } \\
0\end{array}$ & $\begin{array}{c}\text { n } \\
\text { N } \\
\text { N } \\
\text { 员 } \\
\text { ñ }\end{array}$ & $\begin{array}{l}8 \\
0 \\
0 \\
0 \\
0 \\
0 \\
0 \\
0\end{array}$ & 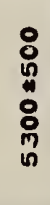 \\
\hline$m$ & 0 & 0 & 0 & 1 & ? & $\stackrel{\bullet}{0}$ & ? & $?$ & 0 & 0 & 0 & 0 & 0 \\
\hline$<$ & $\frac{\vec{n}}{\grave{n}}$ & $\stackrel{m}{\vdots}$ & $\begin{array}{l}a \\
\dot{m} \\
\dot{n}\end{array}$ & $\begin{array}{l}= \\
\vdots \\
\vdots\end{array}$ & 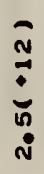 & 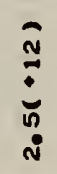 & $\begin{array}{l}a \\
\vdots \\
\vdots \\
0\end{array}$ & $\begin{array}{l}a \\
\vdots\end{array}$ & 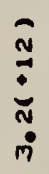 & $\begin{array}{l}= \\
\div \\
0 \\
0\end{array}$ & $\begin{array}{r} \pm \\
\vdots \\
\vdots\end{array}$ & $\vec{\vdots}$ & $\bar{\vdots}$ \\
\hline & $\begin{array}{l}: \\
8 \\
\stackrel{2}{N} \\
\vdots \\
:\end{array}$ & 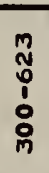 & $\begin{array}{l}\circ \\
8 \\
0 \\
1 \\
0 \\
0 \\
0\end{array}$ & $\stackrel{\omega}{a}$ & & & & $\begin{array}{l}0 \\
8 \\
0 \\
N \\
\vdots \\
0 \\
0 \\
0\end{array}$ & $\begin{array}{l}0 \\
: \\
\stackrel{8}{1} \\
\vdots \\
: \\
:\end{array}$ & 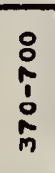 & $\begin{array}{l}0 \\
0 \\
0 \\
0 \\
0 \\
0\end{array}$ & $\begin{array}{l}8 \\
8 \\
\vdots \\
0\end{array}$ & $\begin{array}{l}: \\
\vdots \\
\vdots \\
:\end{array}$ \\
\hline
\end{tabular}

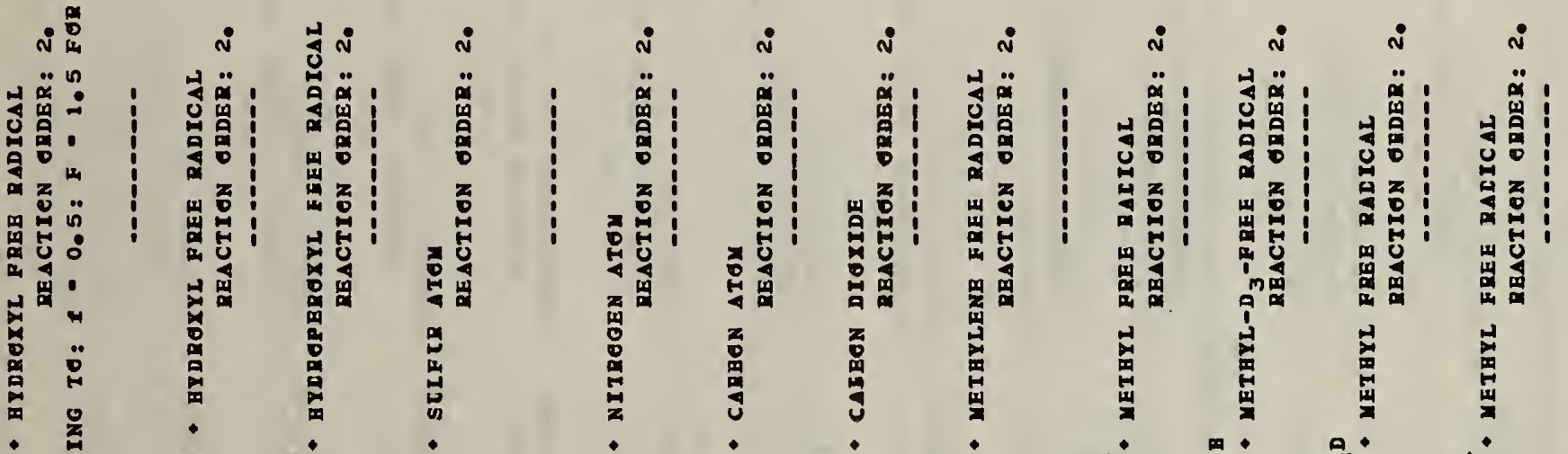

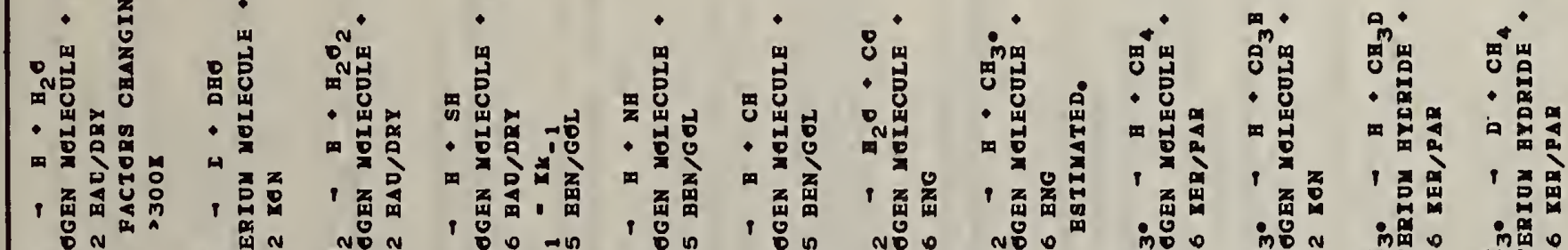

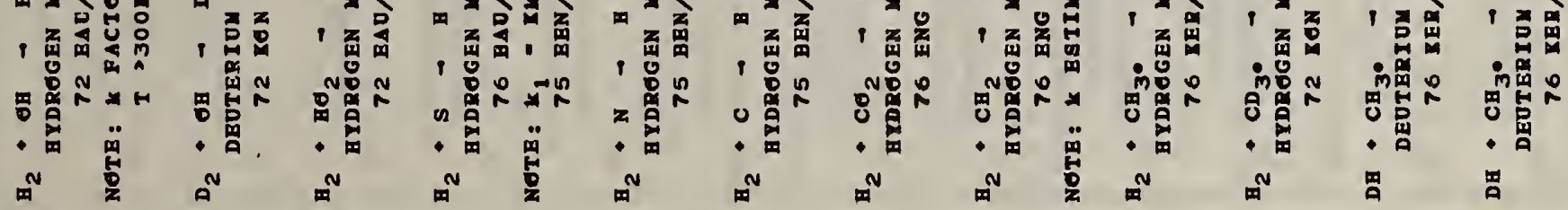




\begin{tabular}{|c|c|c|c|c|c|c|c|c|c|c|c|c|c|c|c|}
\hline 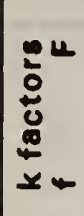 & $\begin{array}{l}\text { : } \\
\vdots\end{array}$ & & & & $\begin{array}{l}: \\
\stackrel{0}{*} \\
:\end{array}$ & & & $\stackrel{0}{0}$ & $\begin{array}{l}: \\
i \\
:\end{array}$ & $\begin{array}{l}: \\
\vdots \\
\vdots\end{array}$ & $\begin{array}{l}\stackrel{0}{:} \\
\stackrel{0}{0}\end{array}$ & $\begin{array}{l}m \\
: \\
\vdots\end{array}$ & & $\begin{array}{ll}\stackrel{0}{0} & : \\
: & : \\
: & 0\end{array}$ & \\
\hline 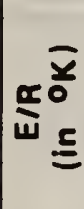 & 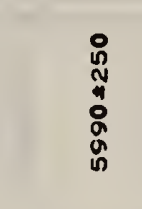 & $\begin{array}{l}: \\
: \\
: \\
: \\
:\end{array}$ & & 号 & $\begin{array}{l}: \\
: \\
0 \\
\vdots \\
: \\
0 \\
\infty \\
0 \\
0\end{array}$ & : & & ' & ० & ० & ' & 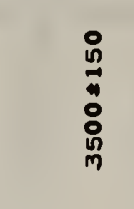 & ० & .0 & 。 \\
\hline$\infty$ & 0 & ० & & 0 & 0 & $\stackrel{0}{0}$ & & , & 0 & 0 & ' & 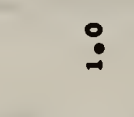 & $\stackrel{n}{0}$ & $\begin{array}{l}\dot{i} \\
\dot{T}\end{array}$ & $:$ \\
\hline$<$ & $\underset{\vdots}{\vdots}$ & ָั & & $\begin{array}{l}\dot{\vdots} \\
\vdots \\
\dot{m}\end{array}$ & 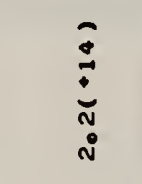 & $\bar{\vdots}$ & & 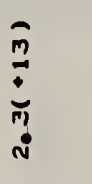 & 矛 & $\begin{array}{c}\tilde{1} \\
\vdots \\
\vdots \\
\vdots\end{array}$ & $\begin{array}{l}\hat{0} \\
\vdots \\
\vdots \\
\dot{m}\end{array}$ & $\begin{array}{l}a \\
\dot{m} \\
\dot{m} \\
\dot{\infty}\end{array}$ & $\begin{array}{l}\bar{\vdots} \\
\vdots \\
\vdots \\
\vdots \\
0\end{array}$ & 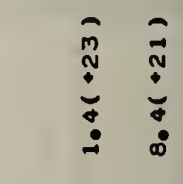 & \\
\hline$\stackrel{Y}{F}$ & $\begin{array}{l}0 \\
\vdots \\
\vdots \\
\vdots \\
m\end{array}$ & $\begin{array}{l}: \\
: \\
\stackrel{0}{0} \\
\vdots \\
\vdots \\
\vdots\end{array}$ & & 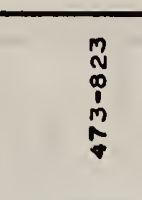 & 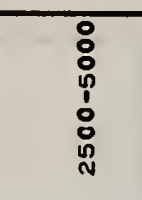 & & & $\stackrel{\circ}{\circ}$ & 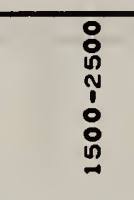 & $\begin{array}{l}0 \\
: \\
\stackrel{0}{N} \\
\vdots \\
\vdots \\
\vdots \\
\end{array}$ & : & $\begin{array}{l} \\
\vdots \\
i \\
\vdots \\
\vdots \\
\vdots\end{array}$ & & $\begin{array}{ll} & \vdots \\
\vdots & \vdots \\
0 & 0 \\
1 & \vdots \\
\vdots & \vdots \\
\vdots & \vdots\end{array}$ & 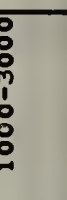 \\
\hline 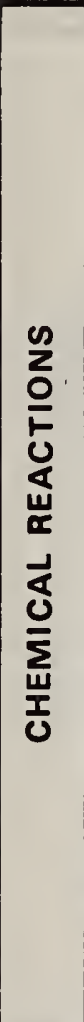 & 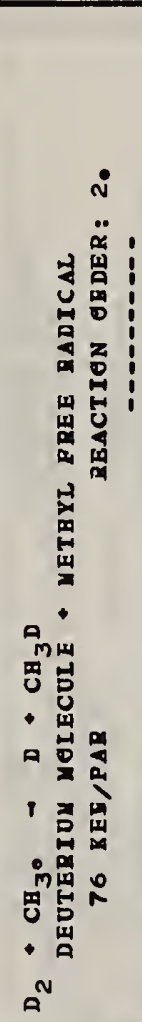 & 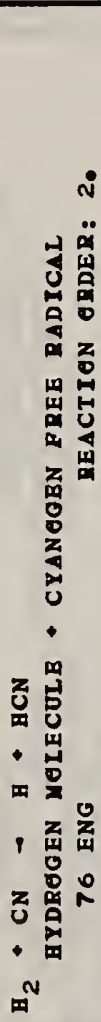 & 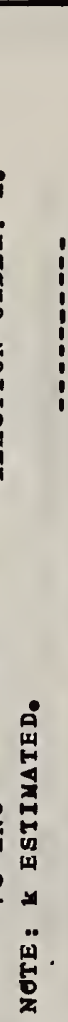 & 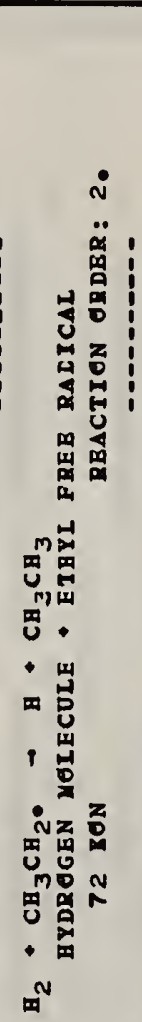 & 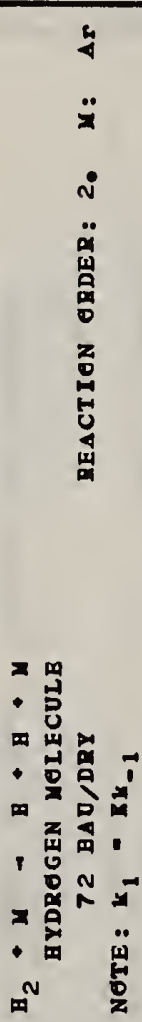 & 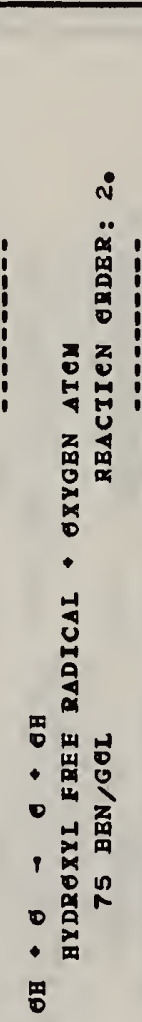 & 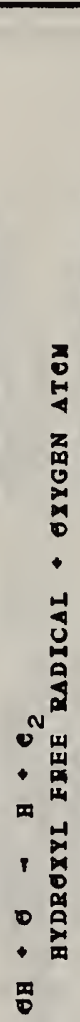 & 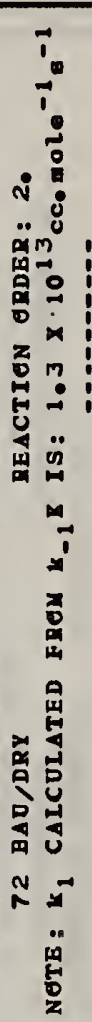 & 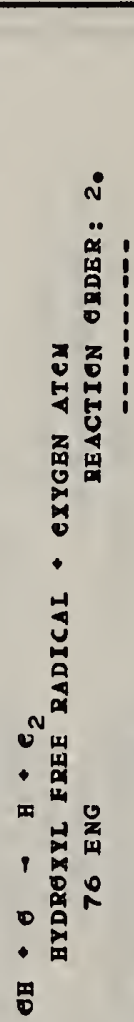 & 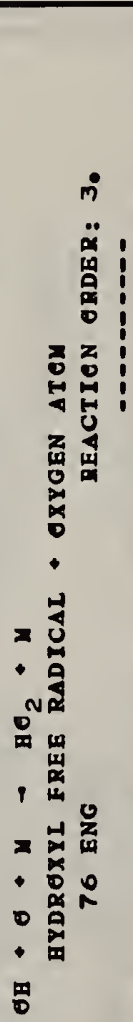 & 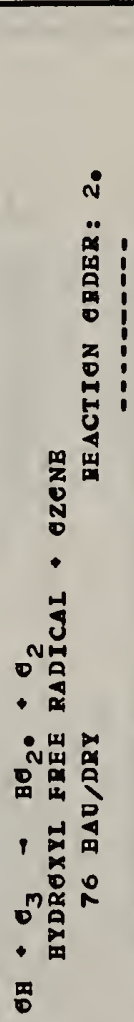 & 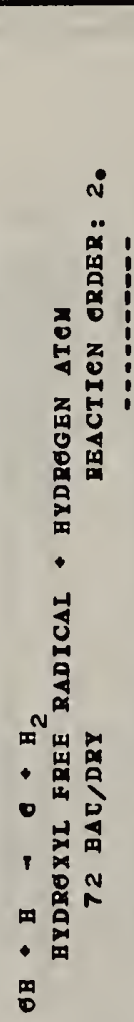 & 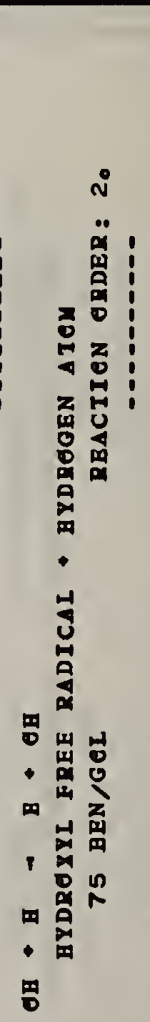 & 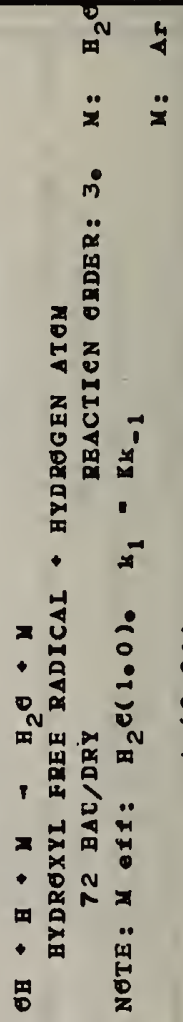 & $\begin{array}{l}\vdots \\
\vdots \\
\vdots \\
\vdots\end{array}$ \\
\hline
\end{tabular}




\begin{tabular}{|c|c|c|c|c|c|c|c|c|c|c|c|c|c|c|c|}
\hline 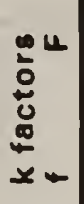 & & $\begin{array}{l}\stackrel{0}{:} \\
\stackrel{\vdots}{ }\end{array}$ & & & $\begin{array}{l}n \\
\text { m. } \\
\stackrel{m}{0}\end{array}$ & $\begin{array}{l}\stackrel{n}{:} \\
\stackrel{0}{0}\end{array}$ & $\begin{array}{l}\stackrel{0}{i} \\
\stackrel{0}{0}\end{array}$ & & & $\begin{array}{l}\stackrel{2}{:} \\
\stackrel{\infty}{:}\end{array}$ & $\begin{array}{l}\tilde{m} \\
\dot{m} \\
\stackrel{m}{0}\end{array}$ & $\begin{array}{l}\stackrel{N}{0} \\
\dot{m} \\
\stackrel{m}{0}\end{array}$ & & & \\
\hline 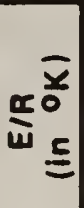 & 0 & ' & ڤ̊ & & $\begin{array}{l}\stackrel{0}{0} \\
\stackrel{\leftrightarrow}{0} \\
\vdots \\
\vdots\end{array}$ & ' & ' & $\begin{array}{l}: \\
\vdots \\
\vdots \\
\vdots\end{array}$ & 0 & 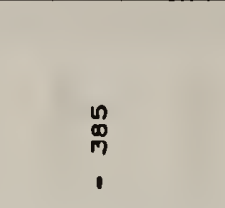 & $\begin{array}{l}: \\
\stackrel{0}{0} \\
0 \\
\vdots \\
: \\
:\end{array}$ & $\begin{array}{l}\text { : } \\
\stackrel{0}{0} \\
0 \\
\vdots \\
\vdots\end{array}$ & $\overrightarrow{\vec{N}}$ & & \\
\hline$\infty$ & $\stackrel{n}{0}$ & ' & 0 & & $\stackrel{n}{\circ}$ & ' & ' & $\stackrel{n}{:}$ & $\stackrel{n}{0}$ & $\stackrel{m}{=}$ & $\stackrel{n}{0}$ & $\stackrel{n}{:}$ & ○ & & \\
\hline$<$ & $\begin{array}{l}\tilde{n} \\
\vdots \\
\vdots\end{array}$ & $\begin{array}{l}\tilde{T} \\
\vdots \\
\dot{b}\end{array}$ & $\begin{array}{l}\tilde{m} \\
\dot{\vec{N}} \\
\dot{m}\end{array}$ & & $\begin{array}{l}\vdots \\
\vdots \\
\vdots \\
\dot{0}\end{array}$ & 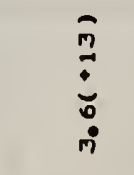 & $\begin{array}{l}\dot{0} \\
\dot{\vdots} \\
\dot{\infty}\end{array}$ & $\bar{\vdots}$ & $\begin{array}{l}\bar{\vdots} \\
\vdots \\
\dot{m} \\
\dot{0}\end{array}$ & $\dot{a}$ & $\begin{array}{l}\vdots \\
\vdots \\
\vdots\end{array}$ & $\begin{array}{l}\bar{\vdots} \\
\vdots \\
\dot{0}\end{array}$ & $\begin{array}{l}\hat{m} \\
\dot{\Xi} \\
\dot{0}\end{array}$ & & \\
\hline$\stackrel{Y}{F}$ & 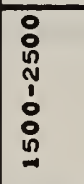 & 品 & 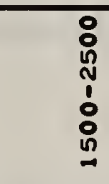 & & 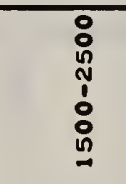 & $:$ & $\stackrel{\circ}{\circ}$ & & & 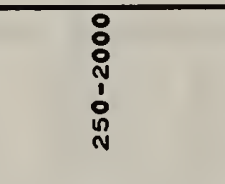 & & 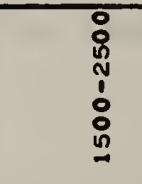 & $\begin{array}{l}\mathbf{0} \\
\stackrel{\circ}{n} \\
\vdots \\
\vdots \\
\vdots \\
\vdots\end{array}$ & & \\
\hline 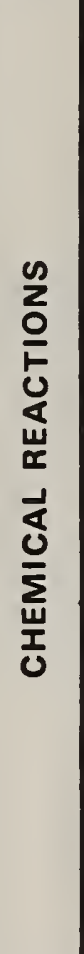 & 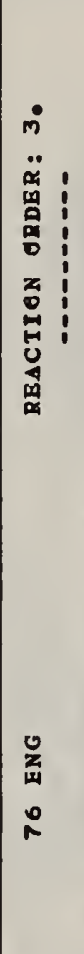 & 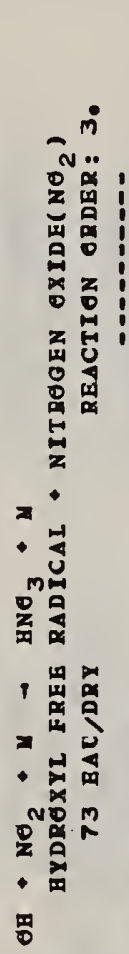 & 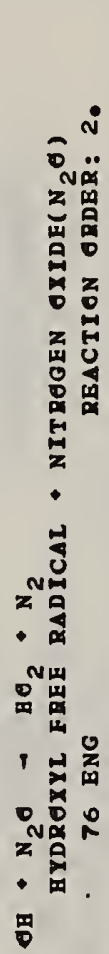 & 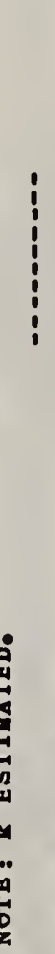 & 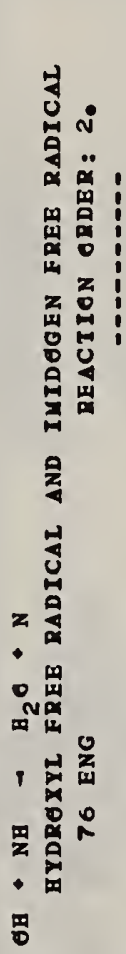 & 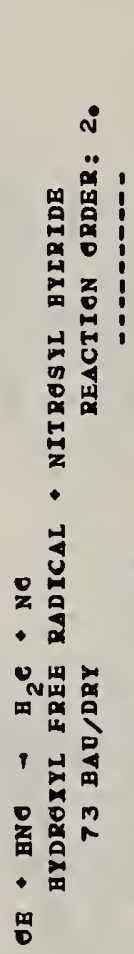 & 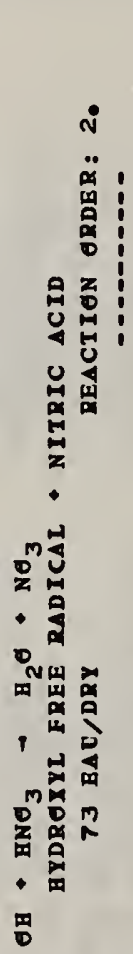 & 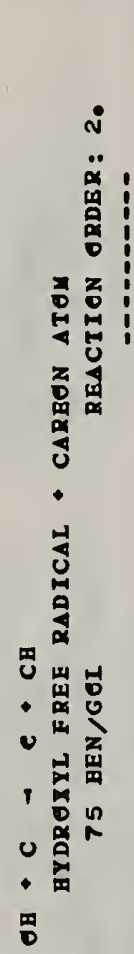 & 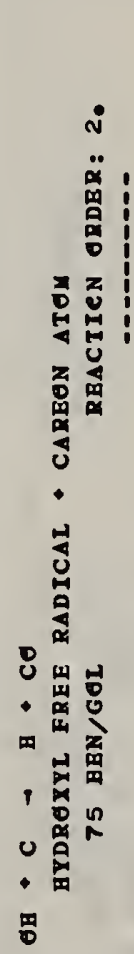 & 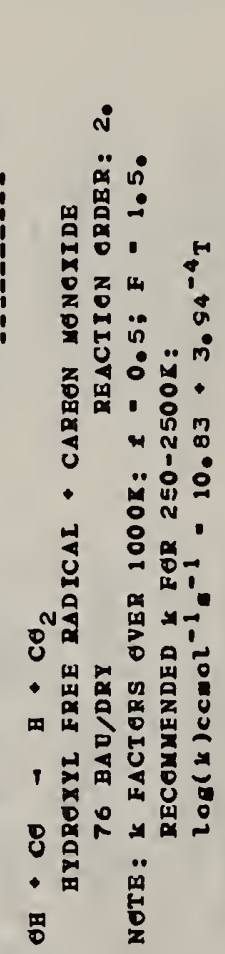 & 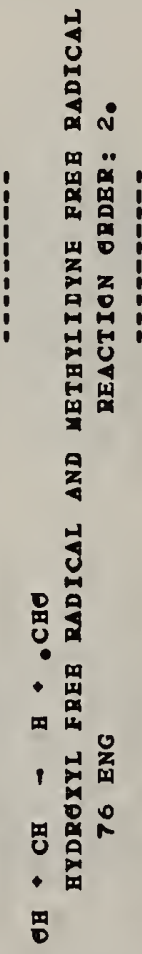 & 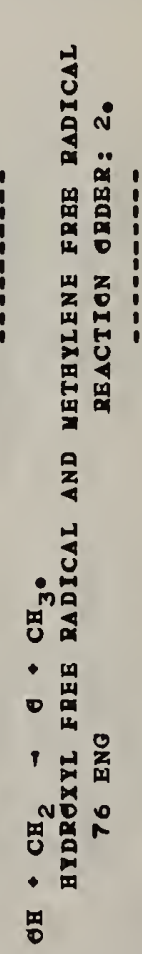 & 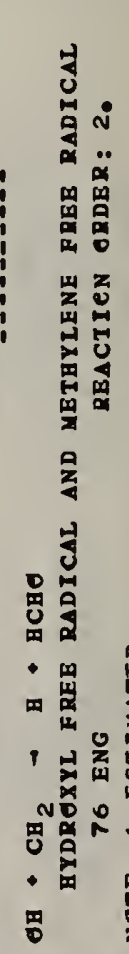 & 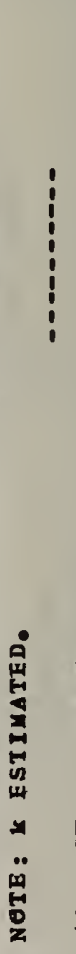 & 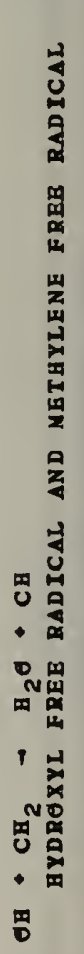 \\
\hline
\end{tabular}




\begin{tabular}{|c|c|c|c|c|c|c|c|c|c|c|c|c|c|}
\hline 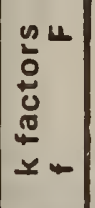 & $\begin{array}{l}\stackrel{N}{m} \\
m \\
\stackrel{0}{\circ}\end{array}$ & & $\begin{array}{l}\text { m } \\
\dot{m} \\
m \\
\dot{0}\end{array}$ & $\begin{array}{l}: \\
\text { i } \\
:\end{array}$ & & & $\begin{array}{l}: \\
\text { a } \\
:\end{array}$ & & $\begin{array}{l}\text { m } \\
\dot{\circ} \\
\stackrel{m}{\circ}\end{array}$ & $\begin{array}{l}\vec{N} \\
\ddot{0}\end{array}$ & $\begin{array}{l}+ \\
:\end{array}$ & $\begin{array}{l}m \\
= \\
\infty \\
\bullet\end{array}$ & \\
\hline 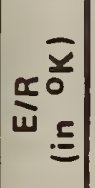 & $\begin{array}{l}: \\
\stackrel{\leftrightarrow}{N} \\
\stackrel{N}{*} \\
: \\
: \\
\dot{0}\end{array}$ & $\circ$ & $\begin{array}{l}: \\
: \\
\stackrel{0}{N} \\
: \\
: \\
:\end{array}$ & 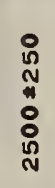 & 号 & \begin{tabular}{l}
$:$ \\
$\stackrel{\circ}{n}$ \\
\hdashline \\
0
\end{tabular} & \begin{tabular}{l}
$:$ \\
$:$ \\
\hdashline \\
\hdashline
\end{tabular} & 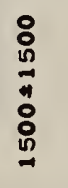 & 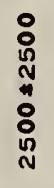 & 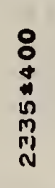 & 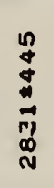 & 1 & 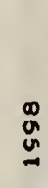 \\
\hline$\infty$ & $\stackrel{n}{:}$ & $\circ$ & 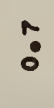 & 0 & $:$ & 0 & : & 0 & $\because$ & 0 & 0 & ' & 0 \\
\hline$<$ & $\begin{array}{l}\overline{7} \\
\vdots \\
0\end{array}$ & 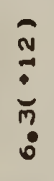 & $\frac{0}{\vdots}$ & $\begin{array}{l}\hat{m} \\
\dot{m} \\
\dot{N} \\
\dot{m}\end{array}$ & $\begin{array}{l}\hat{0} \\
\vdots \\
\dot{n}\end{array}$ & 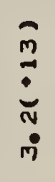 & 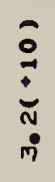 & $\begin{array}{l}\tilde{N} \\
\stackrel{N}{N} \\
\stackrel{0}{*}\end{array}$ & $\begin{array}{l}\Xi \\
\vdots \\
\vdots\end{array}$ & $\stackrel{\tilde{y}}{\grave{0}}$ & 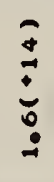 & $\begin{array}{l}\mathfrak{N} \\
\pm \\
\Xi\end{array}$ & 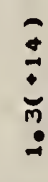 \\
\hline$\stackrel{Y}{F}$ & $\begin{array}{l}: \\
: \\
\stackrel{0}{0} \\
\vdots \\
\vdots \\
0 \\
\vdots\end{array}$ & 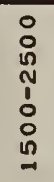 & 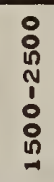 & $\begin{array}{l}: \\
: \\
\stackrel{0}{N} \\
\vdots \\
\vdots \\
:\end{array}$ & $\begin{array}{l}\text { : } \\
\stackrel{n}{N} \\
\vdots \\
\vdots \\
0 \\
0\end{array}$ & $\begin{array}{l}0 \\
: \\
\stackrel{D}{n} \\
\vdots \\
\vdots \\
0 \\
0\end{array}$ & 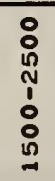 & $\begin{array}{l}0 \\
: \\
\stackrel{\circ}{N} \\
\vdots \\
\vdots \\
0 \\
0\end{array}$ & $\begin{array}{l}0 \\
: \\
\stackrel{0}{0} \\
1 \\
\vdots \\
\vdots \\
0 \\
0\end{array}$ & $\begin{array}{l}: \\
: \\
\stackrel{N}{1} \\
\vdots \\
: \\
\dot{m}\end{array}$ & $\begin{array}{l}0 \\
\vdots \\
\vdots \\
\vdots \\
\vdots \\
0 \\
0 \\
m\end{array}$ & $\stackrel{\circ}{\circ}$ & 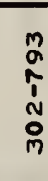 \\
\hline
\end{tabular}

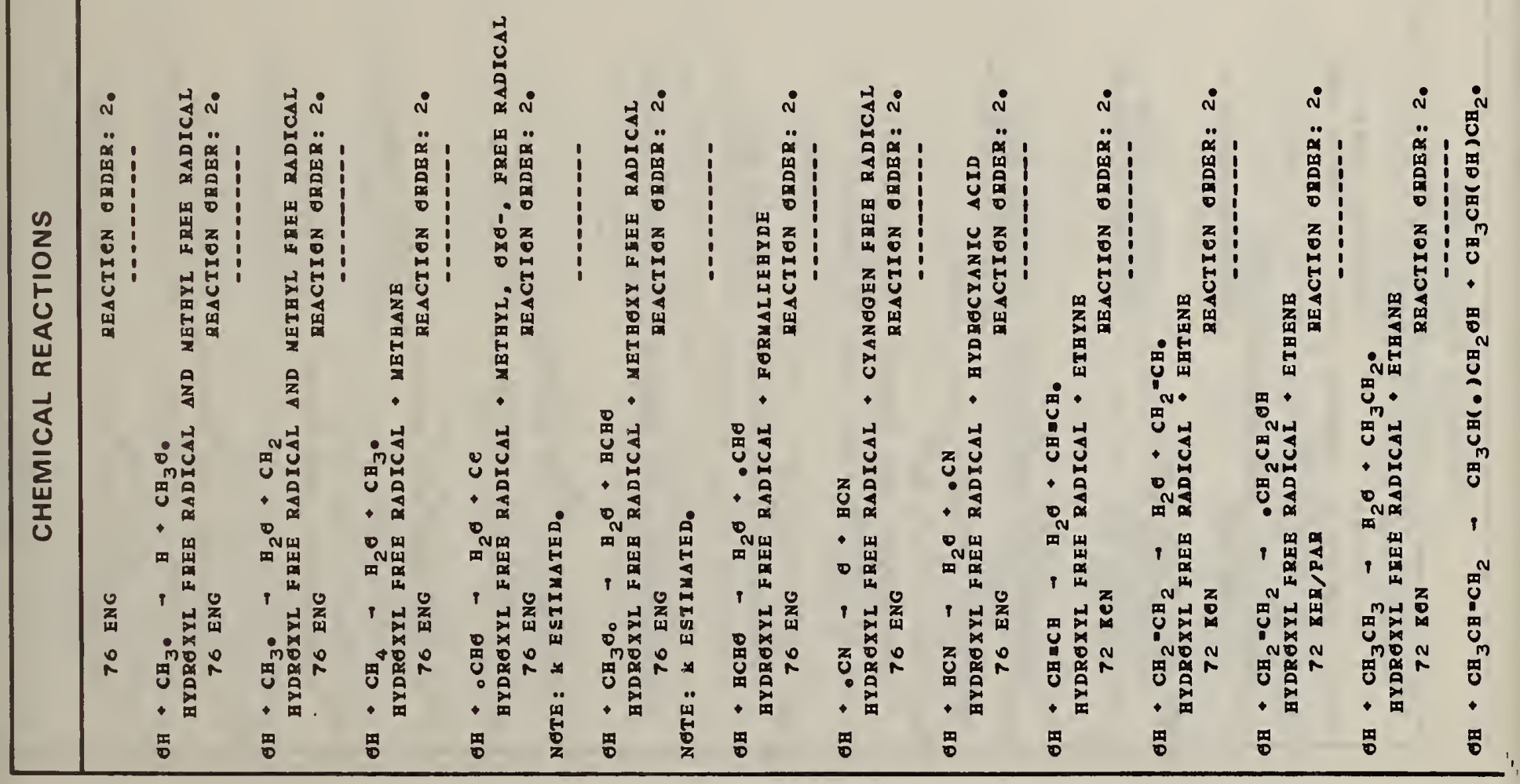




\begin{tabular}{|c|c|c|c|c|c|c|c|c|c|c|c|c|c|c|c|}
\hline 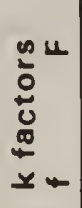 & & $\begin{array}{l}? \\
\because \\
:\end{array}$ & $\begin{array}{l}\text { ก: } \\
\dot{\text { N }} \\
\dot{0}:\end{array}$ & ": & & 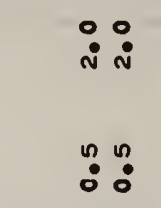 & $\begin{array}{l}: \\
:\end{array}$ & $\begin{array}{l}: \\
\text { N } \\
:\end{array}$ & \% & $\begin{array}{l}: \\
: \\
:\end{array}$ & $\begin{array}{l}? \\
: \\
:\end{array}$ & $\begin{array}{l}\stackrel{N}{:} \\
\because \\
:\end{array}$ & $\begin{array}{l}\stackrel{N}{\circ} \\
m \\
m\end{array}$ & $\begin{array}{l}\dot{m} \\
\dot{m} \\
m \\
:\end{array}$ & \\
\hline $\begin{array}{l}\bar{x} \\
\tilde{w}^{\underline{x}} \\
.\end{array}$ & ' & ' & 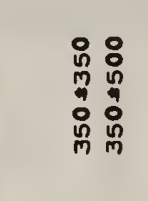 & : & & 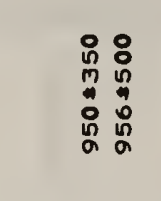 & $\begin{array}{l}: \\
: \\
: \\
: \\
:\end{array}$ & 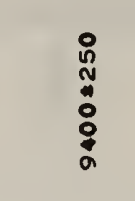 & & : & $\begin{array}{l}: \\
: \\
0 \\
0 \\
0 \\
0 \\
0 \\
0\end{array}$ & ' & $\begin{array}{l}: \\
\stackrel{D}{N} \\
\stackrel{0}{0}\end{array}$ & $\begin{array}{l}: \\
: \\
\vdots \\
: \\
\vdots \\
=\end{array}$ & \\
\hline $\boldsymbol{\infty}$ & ' & ' & $\circ 0$ & 0 & & 00 & 0 & - & & - & - & ' & 0 & 0 & \\
\hline$\alpha$ & $\begin{array}{l}\tilde{N} \\
\vdots \\
\vdots \\
\vdots\end{array}$ & $\begin{array}{l}\infty \\
\vdots \\
\vdots\end{array}$ & 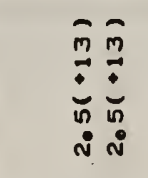 & $\stackrel{5}{\underline{m}}$ & & 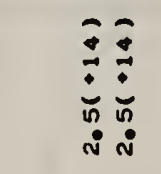 & $\begin{array}{l}\tilde{n} \\
\vdots \\
\vdots\end{array}$ & 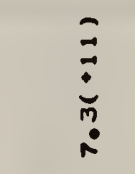 & & in & $\begin{array}{l}\tilde{m} \\
\vdots \\
\dot{0} \\
\text { N }\end{array}$ & $\begin{array}{l}\hat{\infty} \\
\dot{N} \\
\text { ம் }\end{array}$ & 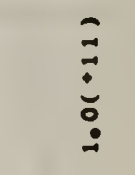 & $\vdots$ & \\
\hline$\stackrel{Y}{\leftarrow}$ & 品 & $\stackrel{\infty}{\stackrel{\infty}{\alpha}}$ & 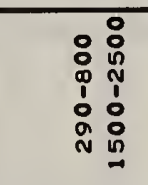 & $\begin{array}{l}0 \\
\vdots \\
\vdots \\
\vdots \\
m\end{array}$ & & 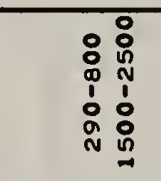 & 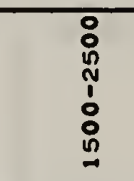 & $\begin{array}{l}: \\
: \\
1 \\
\vdots \\
\circ \\
m\end{array}$ & & $\begin{array}{l}: \\
\vdots \\
\vdots \\
\vdots \\
m\end{array}$ & $\begin{array}{l}: \\
: \\
\$ \\
\vdots \\
: \\
⿱ 亠 凶\end{array}$ & $\stackrel{\circ}{\circ}$ & 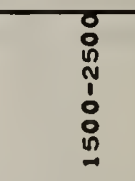 & $\begin{array}{l}: \\
: \\
\vdots \\
\vdots \\
0\end{array}$ & \\
\hline 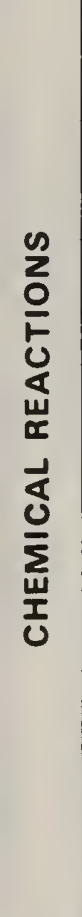 & 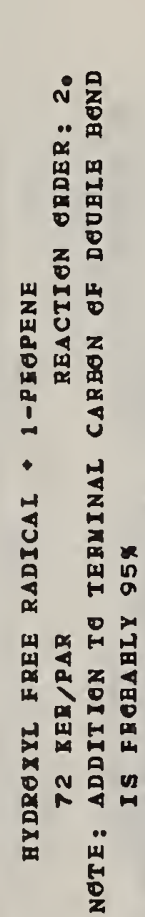 & 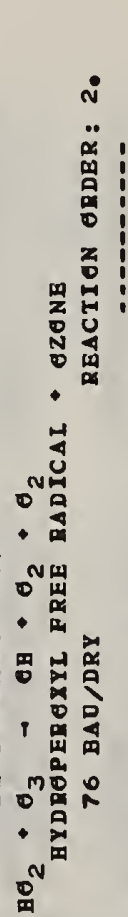 & 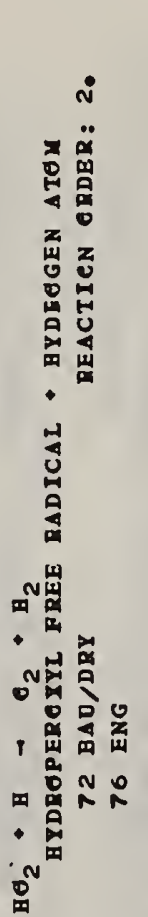 & 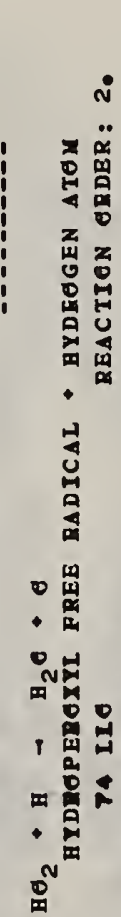 & 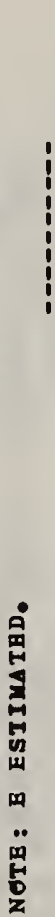 & 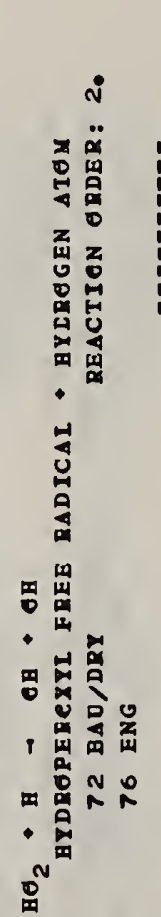 & 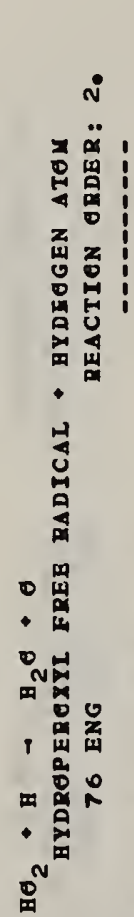 & 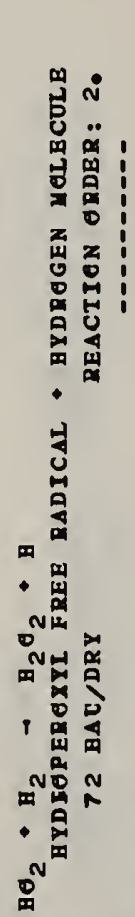 & 然 & 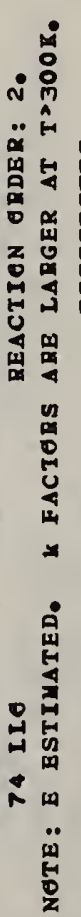 & 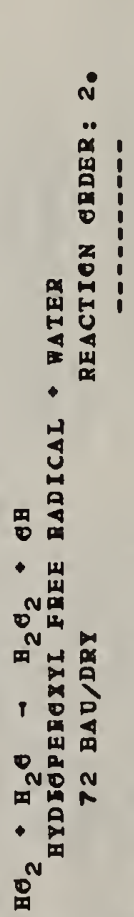 & 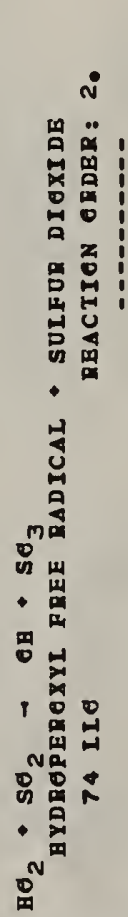 & 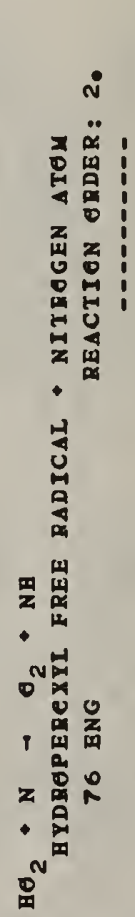 & 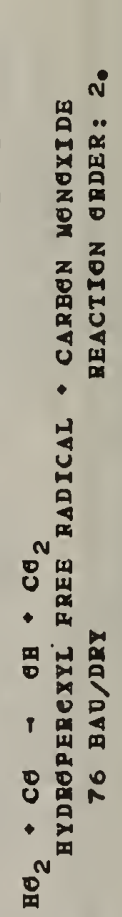 & 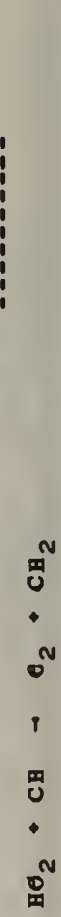 \\
\hline
\end{tabular}




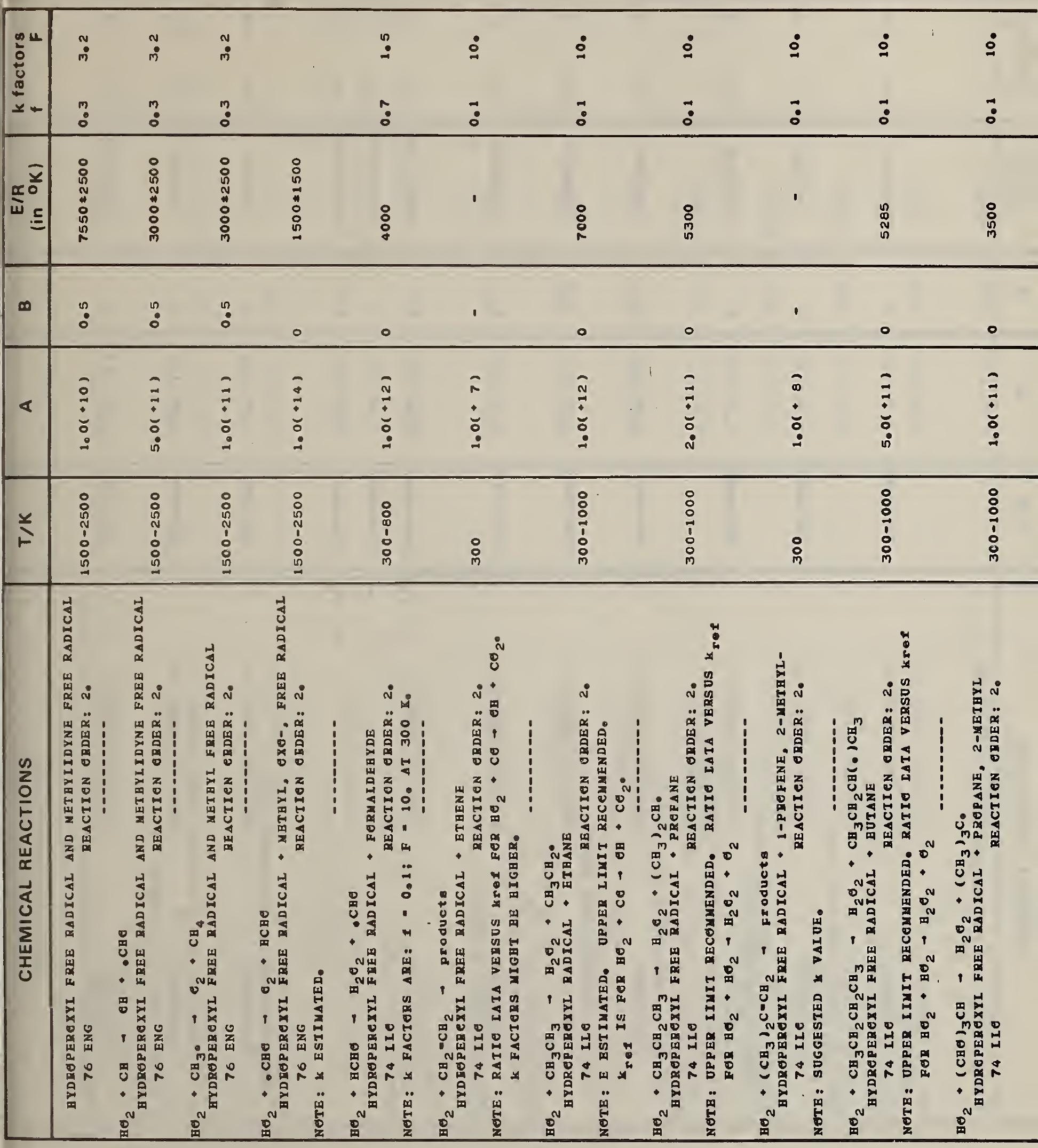




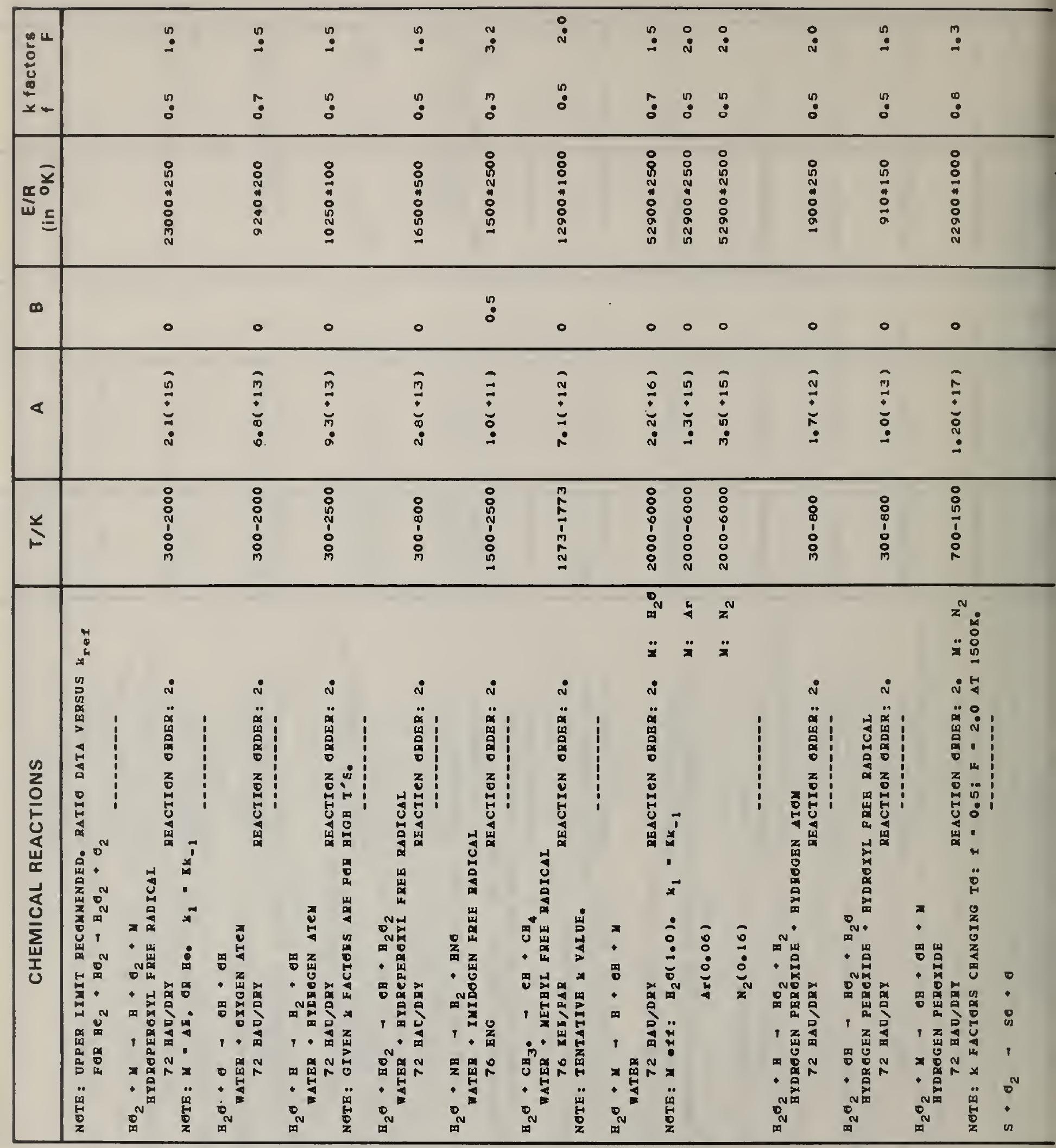




\begin{tabular}{|c|c|c|c|c|c|c|c|c|c|c|c|c|c|}
\hline 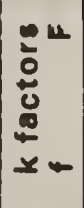 & & & & & & & & & & $\begin{array}{l}\stackrel{n}{:} \\
\stackrel{n}{0}\end{array}$ & $\begin{array}{l}: \\
:\end{array}$ & $\begin{array}{l}\stackrel{0}{\dot{m}} \\
\stackrel{m}{\circ}\end{array}$ & \\
\hline 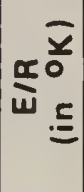 & $\circ$ & $\begin{array}{l}\stackrel{0}{\mathbf{Q}} \\
\stackrel{0}{0}\end{array}$ & 0 & $\begin{array}{l}\stackrel{\circ}{0} \\
\stackrel{0}{0} \\
\stackrel{0}{0}\end{array}$ & $\begin{array}{l}\stackrel{\vdots}{0} \\
\vdots \\
0\end{array}$ & $:$ & ० & $\begin{array}{l}n \\
\vdots \\
\vdots \\
\vdots\end{array}$ & ० & 号 & ' & 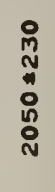 & 号 \\
\hline$\infty$ & $\stackrel{n}{0}$ & $\stackrel{?}{:}$ & $\stackrel{n}{0}$ & $\stackrel{\leftrightarrow}{\circ}$ & $\stackrel{0}{0}$ & $\stackrel{n}{0}$ & $\stackrel{n}{0}$ & $\stackrel{n}{0}$ & $\stackrel{\leftrightarrow}{0}$ & - & ' & ○ & $\stackrel{n}{0}$ \\
\hline$\varangle$ & $\begin{array}{l}\bar{\vdots} \\
\dot{\vec{m}} \\
\dot{0}\end{array}$ & $\begin{array}{l}\vdots \\
\vdots \\
\vdots \\
\dot{0}\end{array}$ & $\begin{array}{l}\bar{\Xi} \\
\vdots \\
\dot{m} \\
:\end{array}$ & $\begin{array}{l}\tilde{y} \\
\vdots \\
\vdots\end{array}$ & $\stackrel{\bar{n}}{\grave{n}}$ & $\underset{\vdots}{\bar{\Xi}}$ & $\underset{\vdots}{\bar{\vdots}}$ & $\stackrel{5}{\vdots}$ & $\underset{\vdots}{\overline{0}}$ & $\stackrel{\tilde{m}}{\grave{n}}$ & $\begin{array}{l}\bar{\vdots} \\
\dot{a} \\
\dot{m}\end{array}$ & $\stackrel{\Xi}{\vdots}$ & $\begin{array}{l}\bar{N} \\
\vdots \\
\vdots \\
\vdots\end{array}$ \\
\hline$\stackrel{y}{F}$ & & & & & & & & & & $\begin{array}{l}0 \\
: \\
0 \\
\vdots \\
\vdots \\
\vdots \\
\vdots \\
0\end{array}$ & $\stackrel{\infty}{\infty}$ & 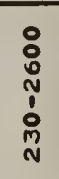 & \\
\hline
\end{tabular}

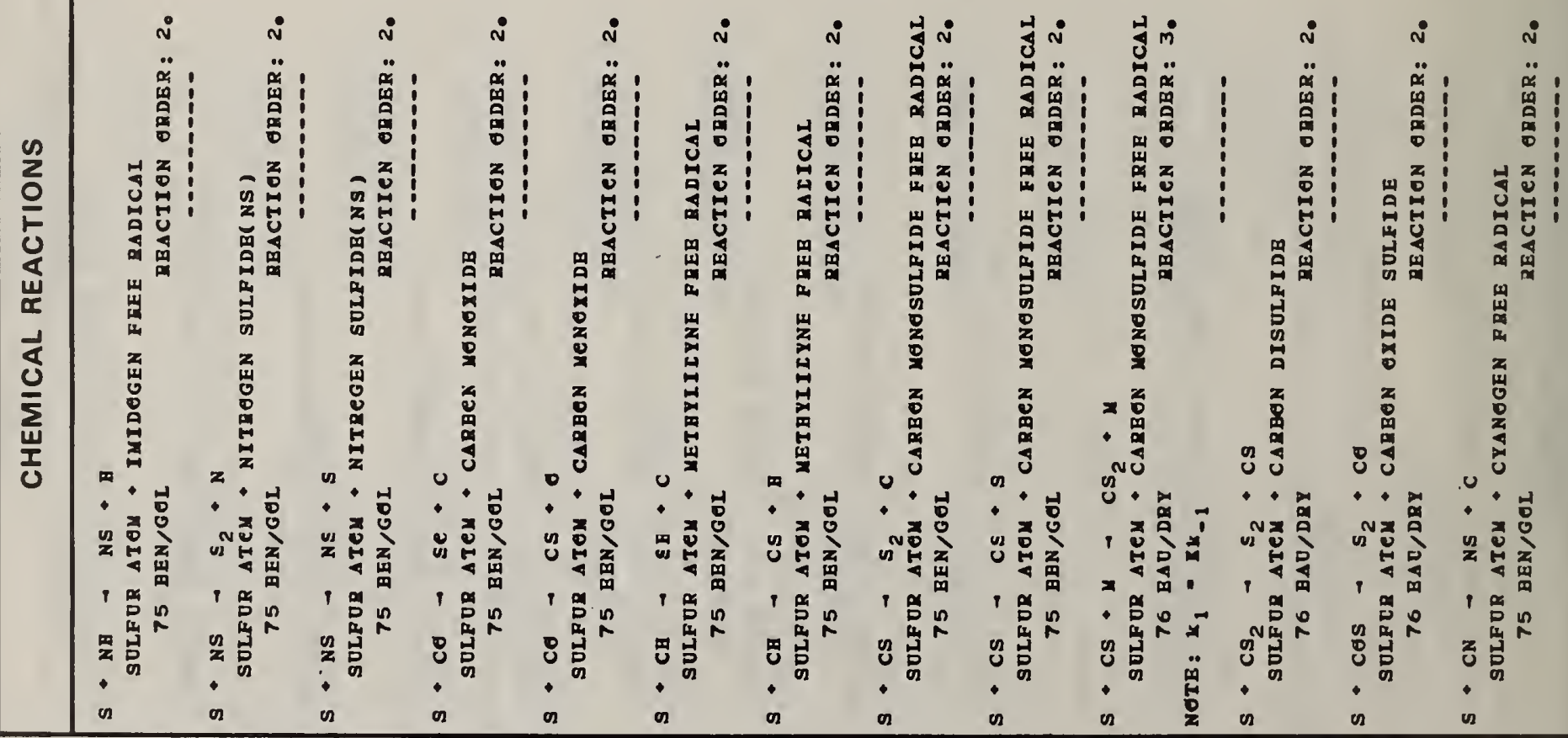




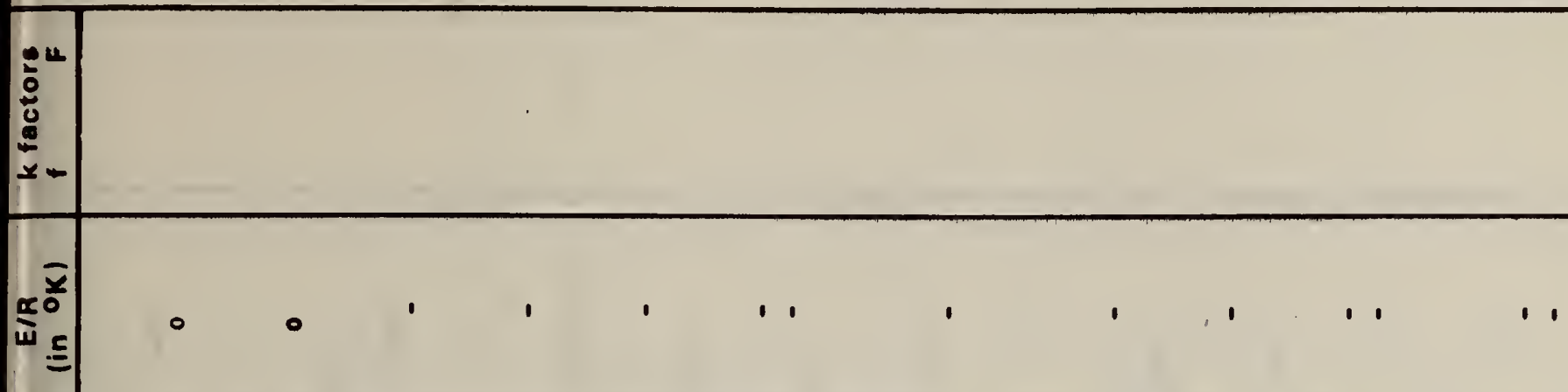

$\infty$

$\because 0 \quad$ : $: 0,1$

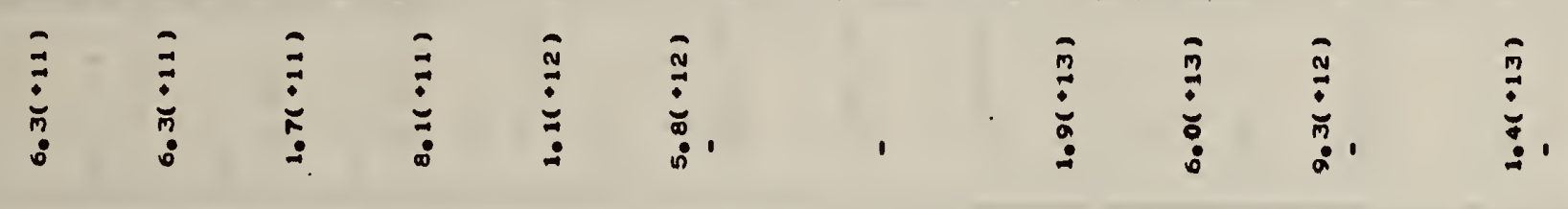

Y

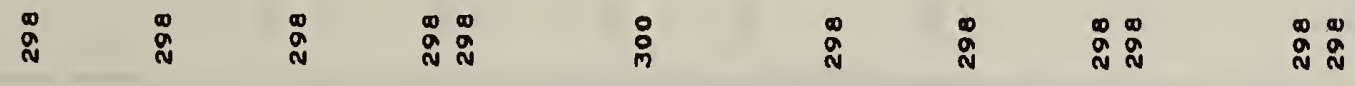

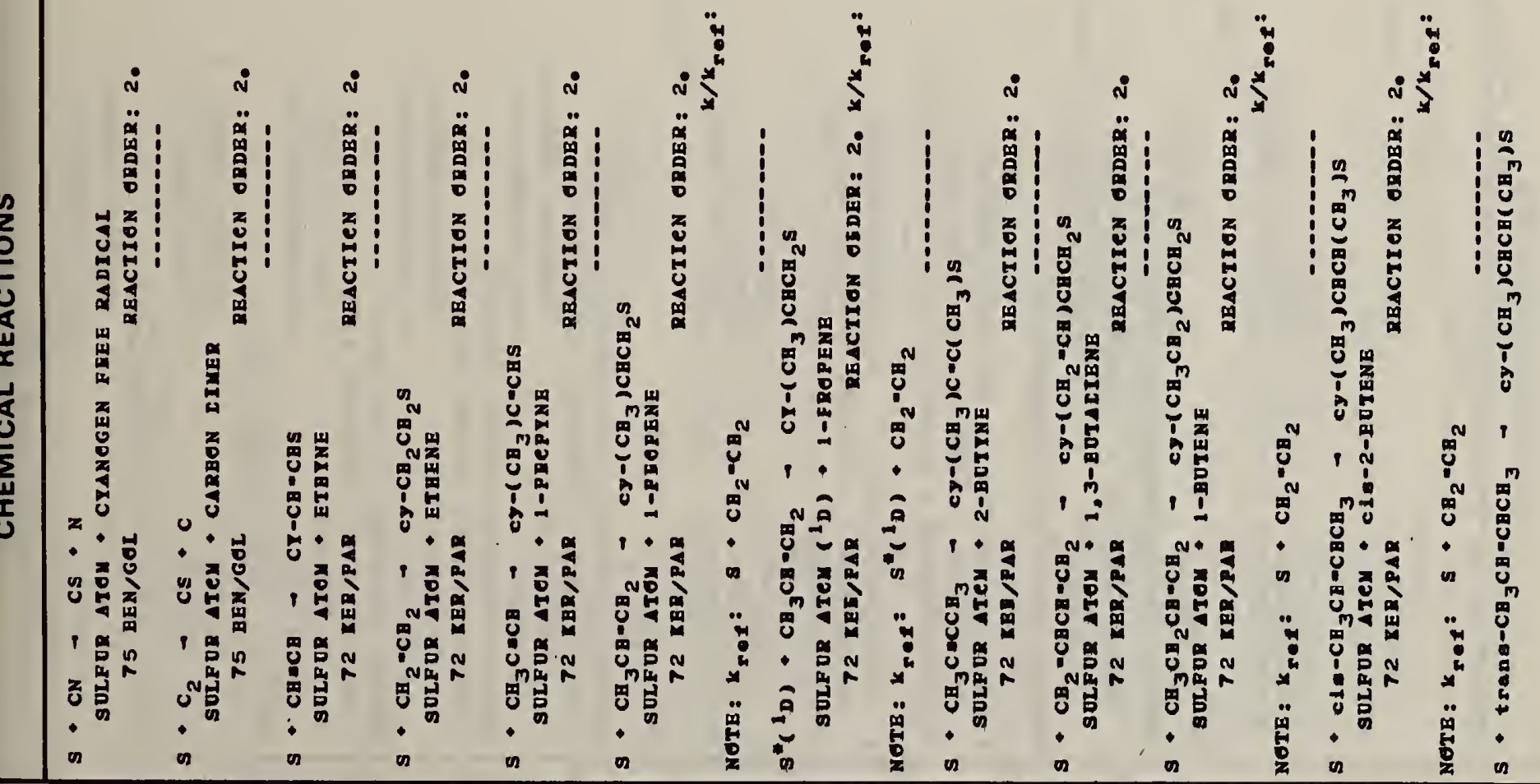




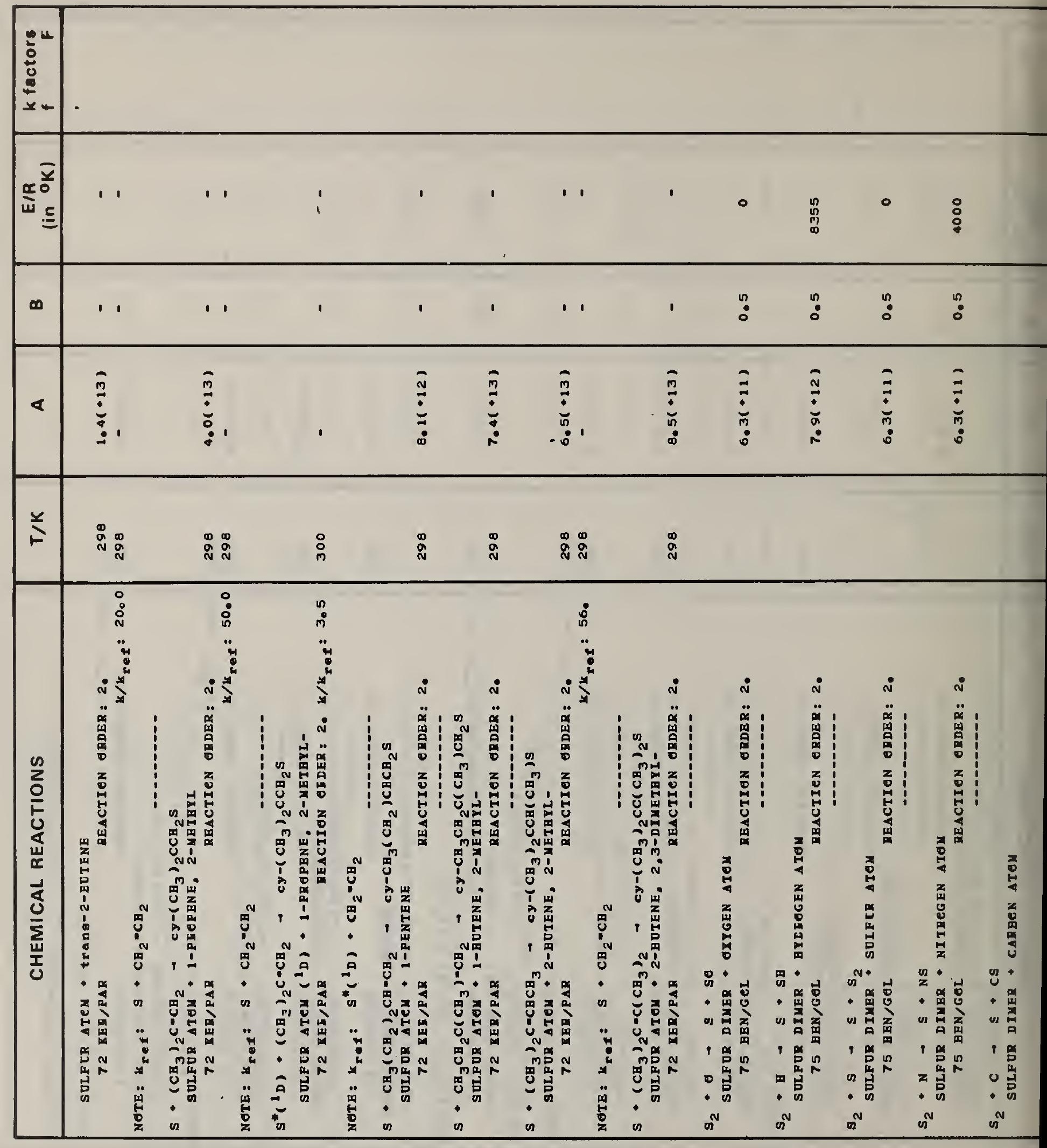




\begin{tabular}{|c|c|c|c|c|c|c|c|c|c|c|c|c|c|c|}
\hline 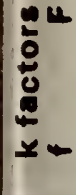 & 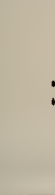 & & & $\begin{array}{l}m \\
:\end{array}$ & $\begin{array}{l}\because \\
:\end{array}$ & & & & & & & & & $\Rightarrow$ \\
\hline$\stackrel{x}{\bar{c}}^{\bar{c}}$ & 0 & - & $\underset{\text { N }}{R}$ & ' & 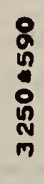 & $\begin{array}{l}\text { 昌 } \\
\text { a } \\
\text { a }\end{array}$ & $\stackrel{8}{\stackrel{8}{8}}$ & $\begin{array}{l}8 \\
2 \\
2\end{array}$ & - & $\underset{\substack{\text { D } \\
\text { d }}}{ }$ & 8 & 0 & 0 & $\begin{array}{l}8 \\
8 \\
0 \\
0\end{array}$ \\
\hline$\infty$ & $:$ & ? & $\stackrel{0}{0}$ & 1 & 0 & ! & ? & ? & $\stackrel{\text { n }}{0}$ & ? & $:$ & $\stackrel{0}{0}$ & : & ? \\
\hline$\alpha$ & $\begin{array}{l}\vec{\vdots} \\
\dot{m} \\
\dot{0}\end{array}$ & $\begin{array}{l}\vdots \\
\vdots \\
\dot{m}\end{array}$ & $\begin{array}{l}\dot{0} \\
\dot{8}\end{array}$ & $\underset{0}{\stackrel{m}{0}}$ & $\vec{\square}$ & $\begin{array}{l}\overrightarrow{6} \\
\dot{m}\end{array}$ & $\stackrel{0}{\vdots}$ & $\begin{array}{l}= \\
\vdots \\
\dot{n}\end{array}$ & $\overrightarrow{0}$ & $\begin{array}{l}\hat{y} \\
\overrightarrow{0}\end{array}$ & $\vec{a}$ & $\vec{b}$ & $\begin{array}{l}\overrightarrow{0} \\
\bar{m} \\
\dot{0}\end{array}$ & 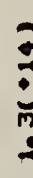 \\
\hline & & & & $\stackrel{\Xi}{\stackrel{\infty}{N}}$ & $\begin{array}{l}0 \\
\dot{0} \\
\dot{1} \\
\dot{0}\end{array}$ & & & & & & & & & : \\
\hline
\end{tabular}

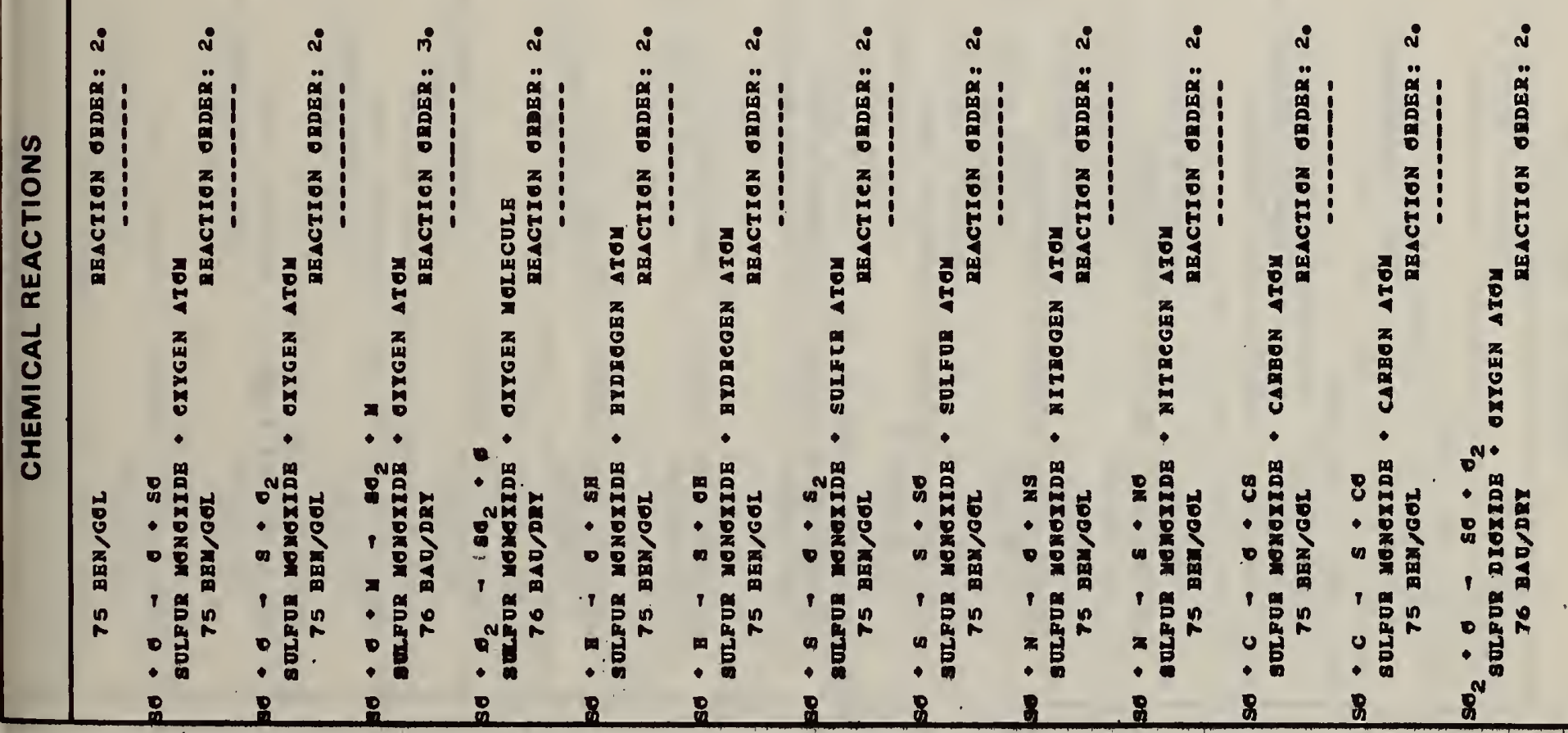




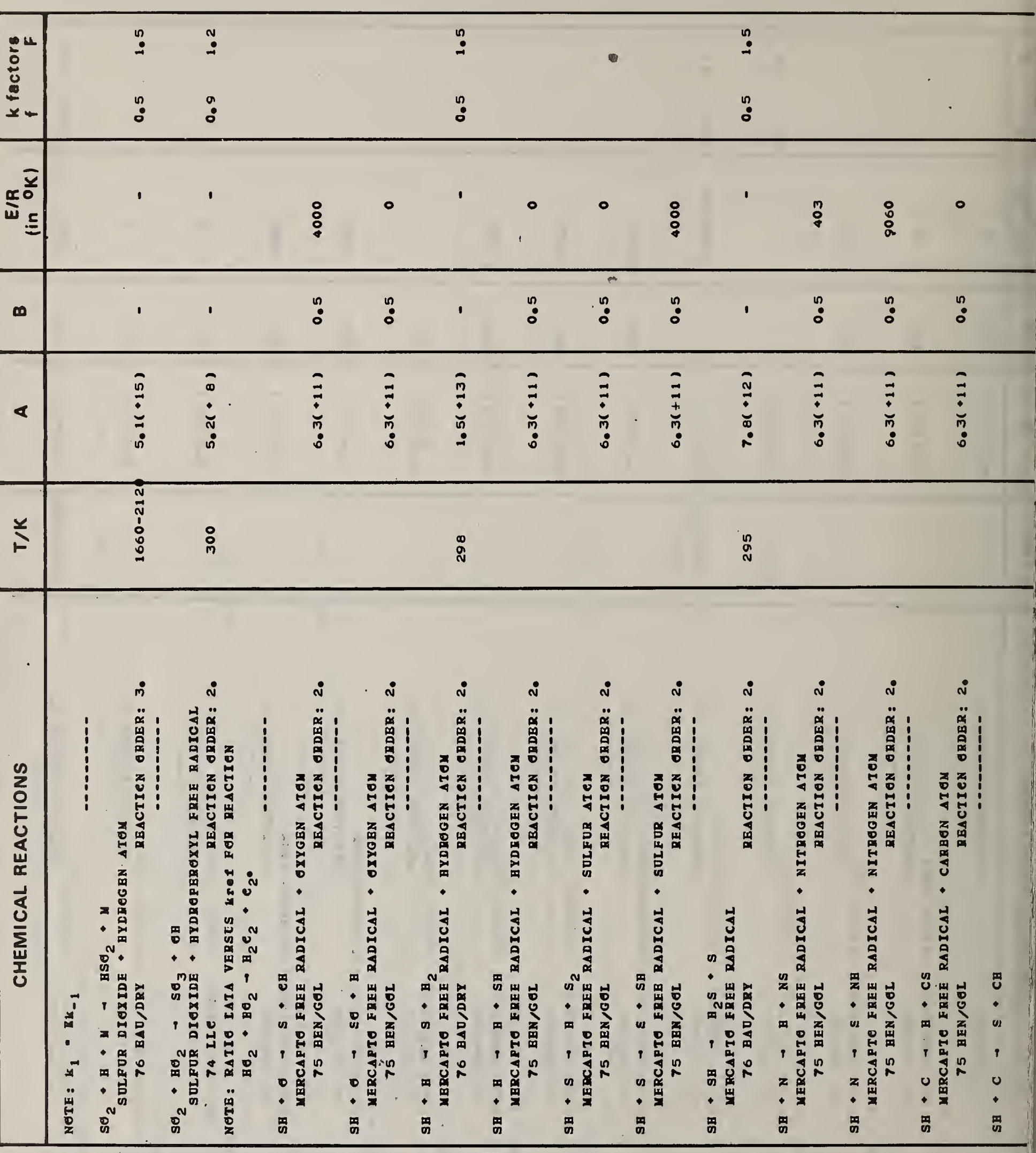




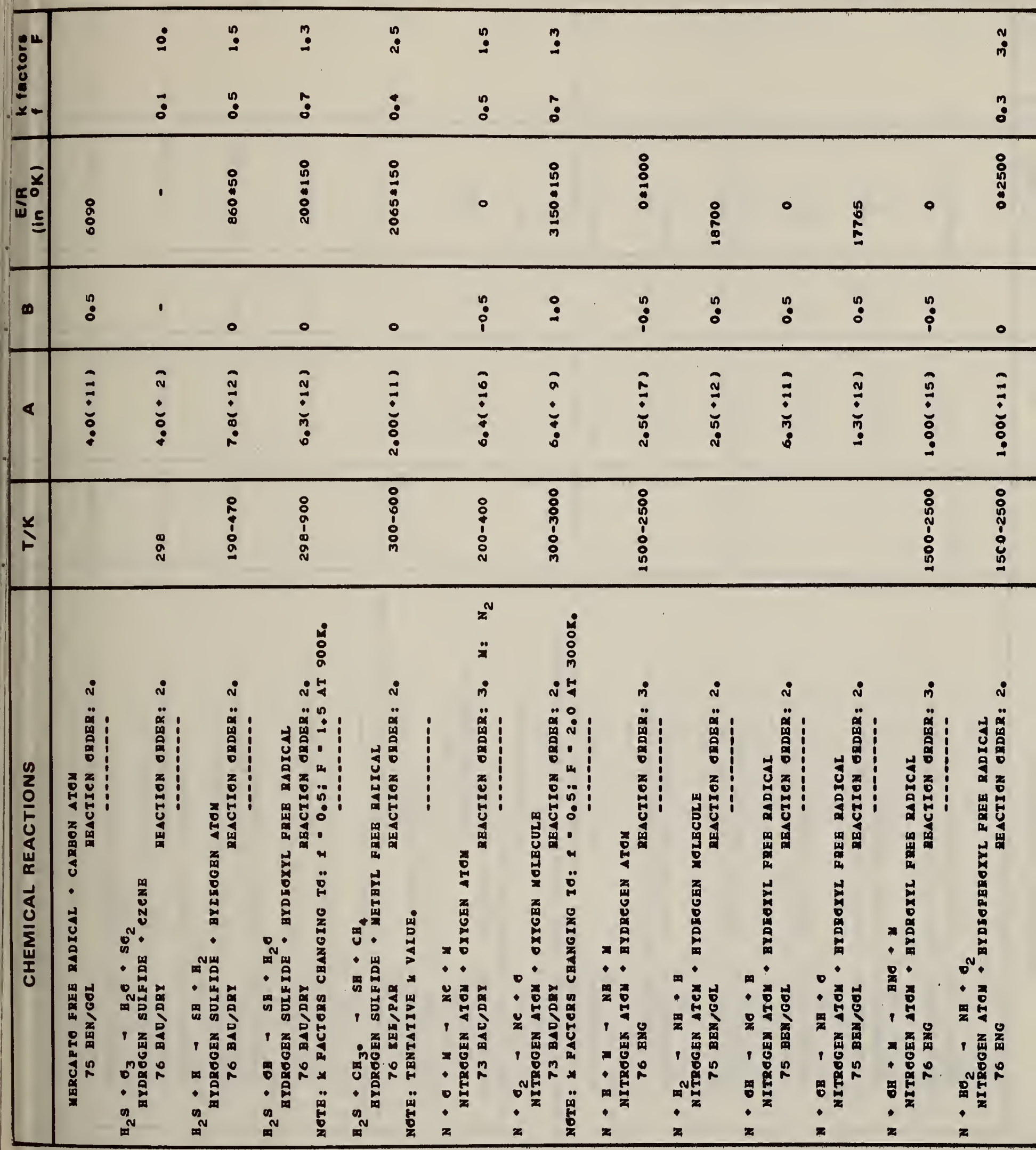




\begin{tabular}{|c|c|c|c|c|c|c|c|c|c|c|c|c|c|c|}
\hline 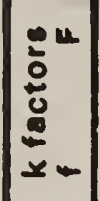 & & & & & & $\begin{array}{l}\stackrel{!}{=} \\
\stackrel{n}{0}\end{array}$ & & & 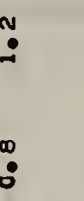 & & & $\begin{array}{l}\stackrel{\circ}{\circ} \\
\stackrel{\circ}{\circ}\end{array}$ & $\begin{array}{l}0 \\
\text { ก } \\
\text { : }\end{array}$ & \\
\hline 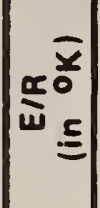 & $\stackrel{\circ}{\vdots}$ & $\stackrel{\circ}{\circ}$ & 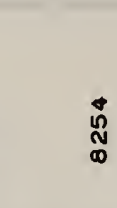 & : & 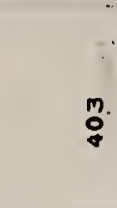 & $\begin{array}{l}0 \\
\vdots \\
\vdots \\
: \\
0 \\
0 \\
1\end{array}$ & $\stackrel{\circ}{\circ}$ & & $\circ$ & $\stackrel{\circ}{:}$ & 离 & 品 & 号 & \\
\hline$\infty$ & $\stackrel{?}{0}$ & $\stackrel{n}{0}$ & $\stackrel{?}{0}$ & $\stackrel{n}{0}$ & in & 0 & $\stackrel{0}{0}$ & ○ & 。 & $\stackrel{?}{0}$ & 0 & $\circ$ & 0 & \\
\hline$<$ & $\begin{array}{l}\vdots \\
\dot{\vdots} \\
\dot{0} \\
\dot{0}\end{array}$ & $\sum_{\bar{\vdots}}$ & $\begin{array}{l}\bar{y} \\
\vdots \\
\vdots\end{array}$ & $\begin{array}{l}\vdots \\
\vdots \\
\dot{n} \\
:\end{array}$ & $\begin{array}{l}\vdots \\
\vdots \\
\dot{m} \\
\dot{0}\end{array}$ & $\begin{array}{l}\vdots \\
\vdots \\
\dot{0} \\
\dot{m}\end{array}$ & $\begin{array}{l}\vdots \\
\vdots \\
\dot{m} \\
:\end{array}$ & $\begin{array}{l}\tilde{m} \\
\vdots \\
\vdots\end{array}$ & 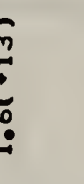 & $\vdots$ & $\begin{array}{l}\hat{N} \\
\stackrel{1}{0} \\
\vdots \\
\vdots\end{array}$ & $\begin{array}{l}\frac{N}{3} \\
\vdots \\
\vdots\end{array}$ & $\begin{array}{l}\widehat{a} \\
\vdots \\
\vdots \\
\vdots\end{array}$ & \\
\hline 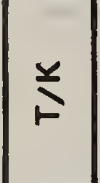 & & & & & & $\begin{array}{l}0 \\
\vdots \\
\vdots \\
\vdots \\
\vdots\end{array}$ & & \begin{tabular}{l}
$:$ \\
$\vdots$ \\
0 \\
0 \\
$\vdots$ \\
$\vdots$ \\
\hdashline
\end{tabular} & 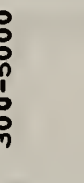 & & 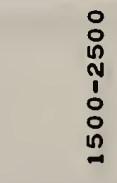 & 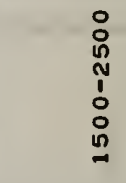 & 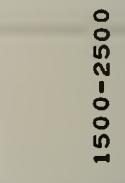 & \\
\hline 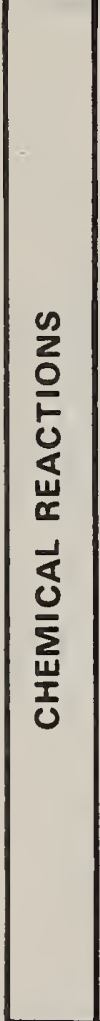 & 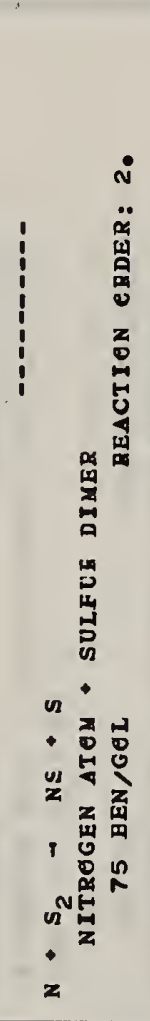 & 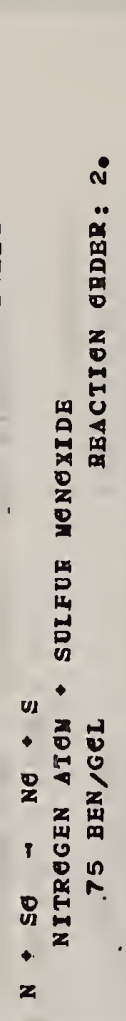 & 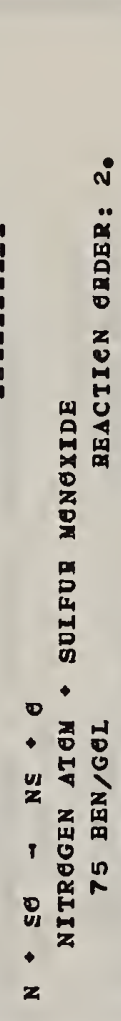 & 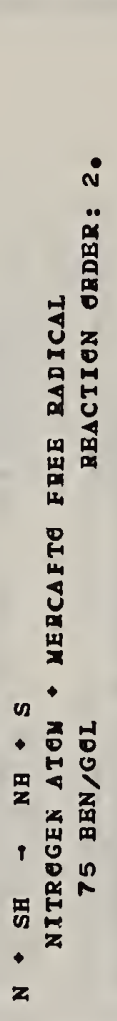 & 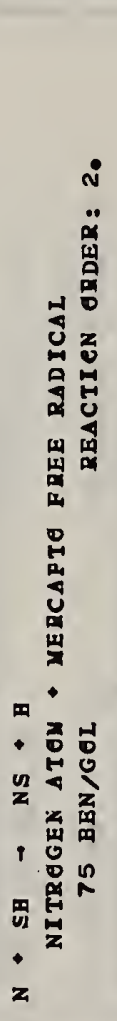 & 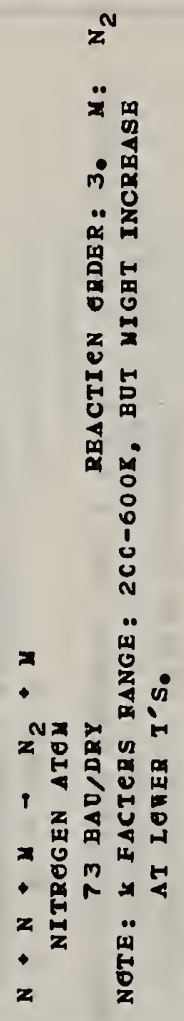 & 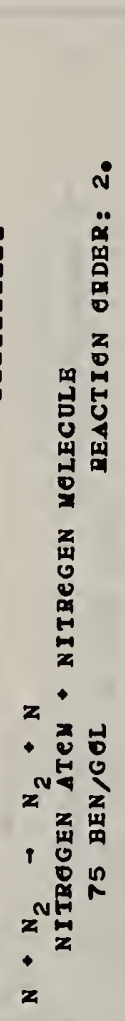 & 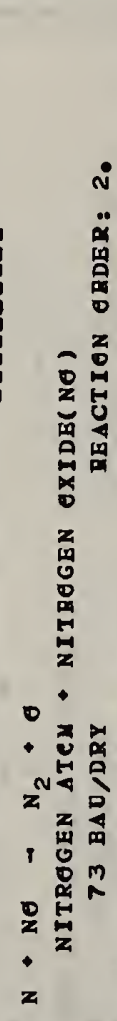 & 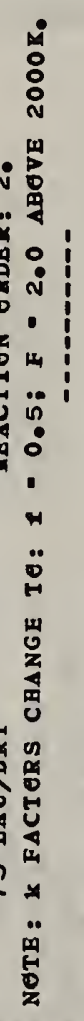 & 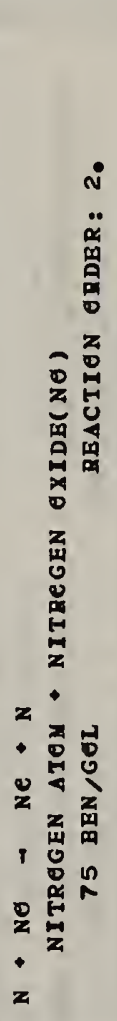 & 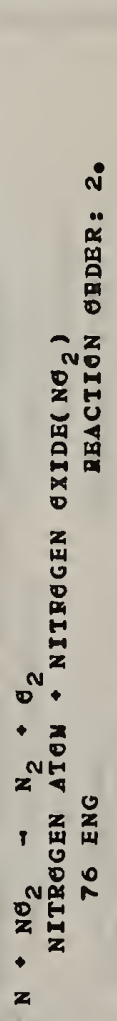 & 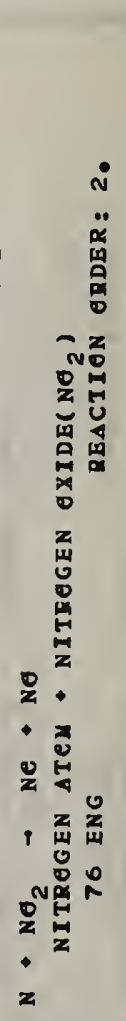 & 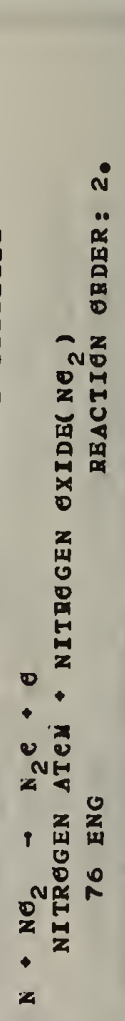 & $\begin{array}{l}z^{N} \\
\dot{z} \\
z \\
i \\
i^{N} \\
z^{N} \\
z^{n}\end{array}$ \\
\hline
\end{tabular}




\begin{tabular}{|c|c|c|c|c|c|c|c|c|c|c|c|c|c|}
\hline $\begin{array}{l}04 \\
\vdots \\
0 \\
0 \\
\vdots \\
\vdots \\
y \\
y\end{array}$ & $\begin{array}{l}\text { m } \\
m \\
m \\
\dot{m}\end{array}$ & & & & & & & & & $\begin{array}{l}\therefore \\
\therefore\end{array}$ & & & $\begin{array}{l}\text { v } \\
\dot{m} \\
m \\
:\end{array}$ \\
\hline 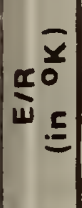 & $\begin{array}{l}: \\
: \\
\text { N } \\
\vdots \\
: \\
: \\
:\end{array}$ & ○ & : & 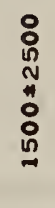 & $\begin{array}{l}\stackrel{8}{0} \\
\stackrel{n}{N} \\
\ddot{0} \\
: \\
\vdots\end{array}$ & 0 & $:$ & $\begin{array}{l}: \\
\stackrel{p}{n} \\
\text { in }\end{array}$ & $\begin{array}{l}: \\
8 \\
\infty \\
\infty \\
0 \\
m\end{array}$ & $\begin{array}{l}: \\
: \\
: \\
: \\
: \\
:\end{array}$ & $\begin{array}{l}0 \\
\alpha \\
0 \\
0\end{array}$ & 0 & 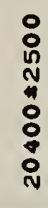 \\
\hline$\infty$ & 0 & $\stackrel{0}{\circ}$ & $\stackrel{n}{0}$ & $\stackrel{n}{:}$ & $\stackrel{\text { ! }}{0}$ & $\stackrel{n}{0}$ & $\stackrel{0}{0}$ & $\stackrel{n}{0}$ & $:$ & $\stackrel{n}{0}$ & ?: & $\stackrel{n}{0}$ & ¿̊ \\
\hline $1<$ & $\begin{array}{l}\tilde{\infty} \\
\dot{0} \\
\dot{0}\end{array}$ & $\begin{array}{l}\overline{7} \\
\dot{m} \\
\dot{0}\end{array}$ & $\bar{\Xi}$ & $\begin{array}{l}0 \\
\vdots \\
\check{0}\end{array}$ & $\begin{array}{l}\bar{\vdots} \\
\vdots \\
\vdots\end{array}$ & $\begin{array}{l}\overline{7} \\
\dot{m} \\
\dot{0}\end{array}$ & $\bar{\Xi}$ & $\begin{array}{l}\tilde{N} \\
\stackrel{0}{0} \\
\text { in }\end{array}$ & $\begin{array}{l}\bar{N} \\
\overrightarrow{\tilde{N}} \\
\dot{m}\end{array}$ & $\begin{array}{l}\bar{\Xi} \\
\vdots \\
\vdots\end{array}$ & $\begin{array}{l}\tilde{N} \\
\stackrel{4}{0} \\
\vdots \\
:\end{array}$ & $\begin{array}{l}\bar{\vdots} \\
\dot{m} \\
\dot{0}\end{array}$ & $\bar{\vdots}$ \\
\hline ㄱ & $\begin{array}{l}\text { : } \\
\stackrel{\leftrightarrow}{n} \\
\vdots \\
\vdots \\
\stackrel{0}{0}\end{array}$ & & & $\begin{array}{l}\circ \\
\stackrel{\circ}{n} \\
\stackrel{1}{n} \\
\vdots \\
\vdots \\
0 \\
0\end{array}$ & 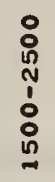 & & & & & $\begin{array}{l}: \\
\stackrel{8}{0} \\
1 \\
\vdots \\
: \\
:\end{array}$ & & & 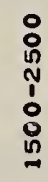 \\
\hline
\end{tabular}

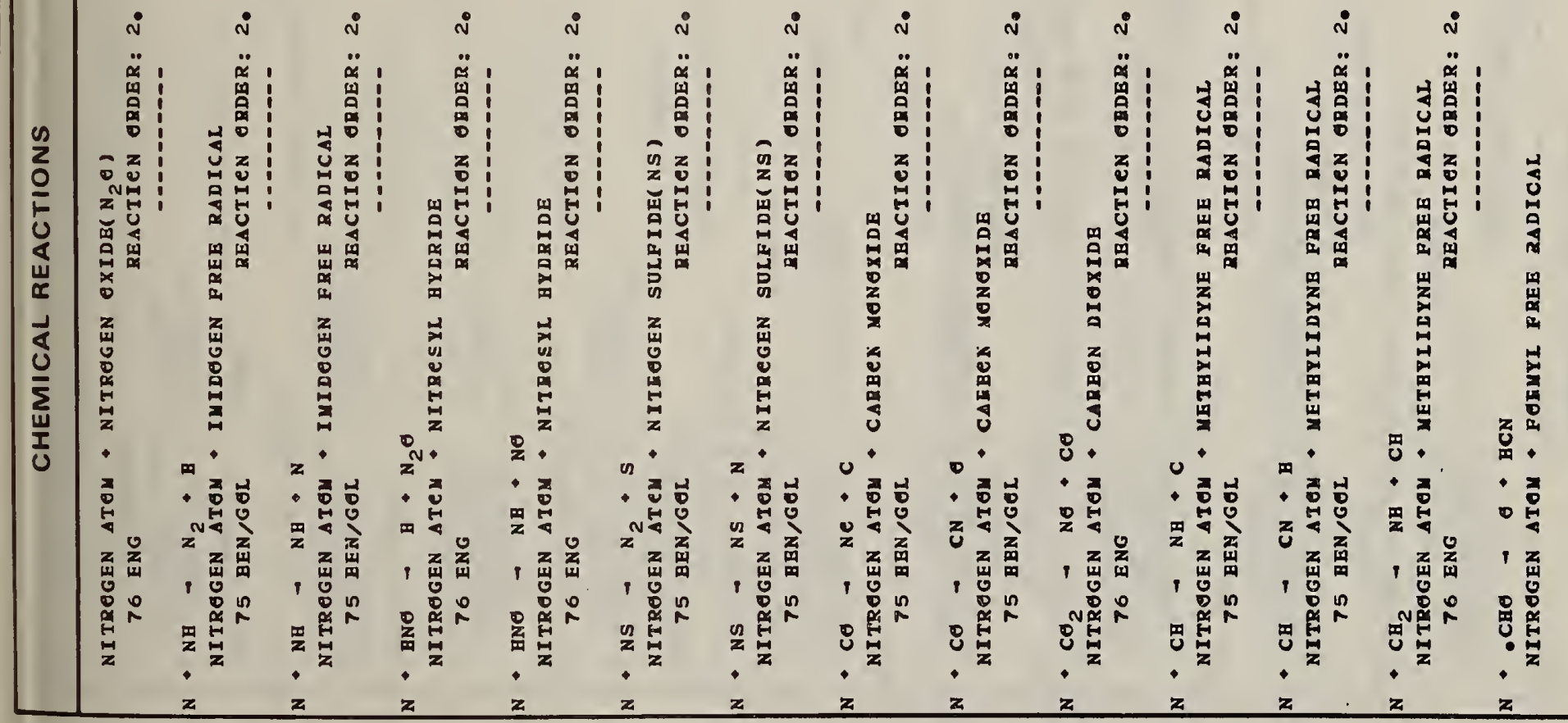




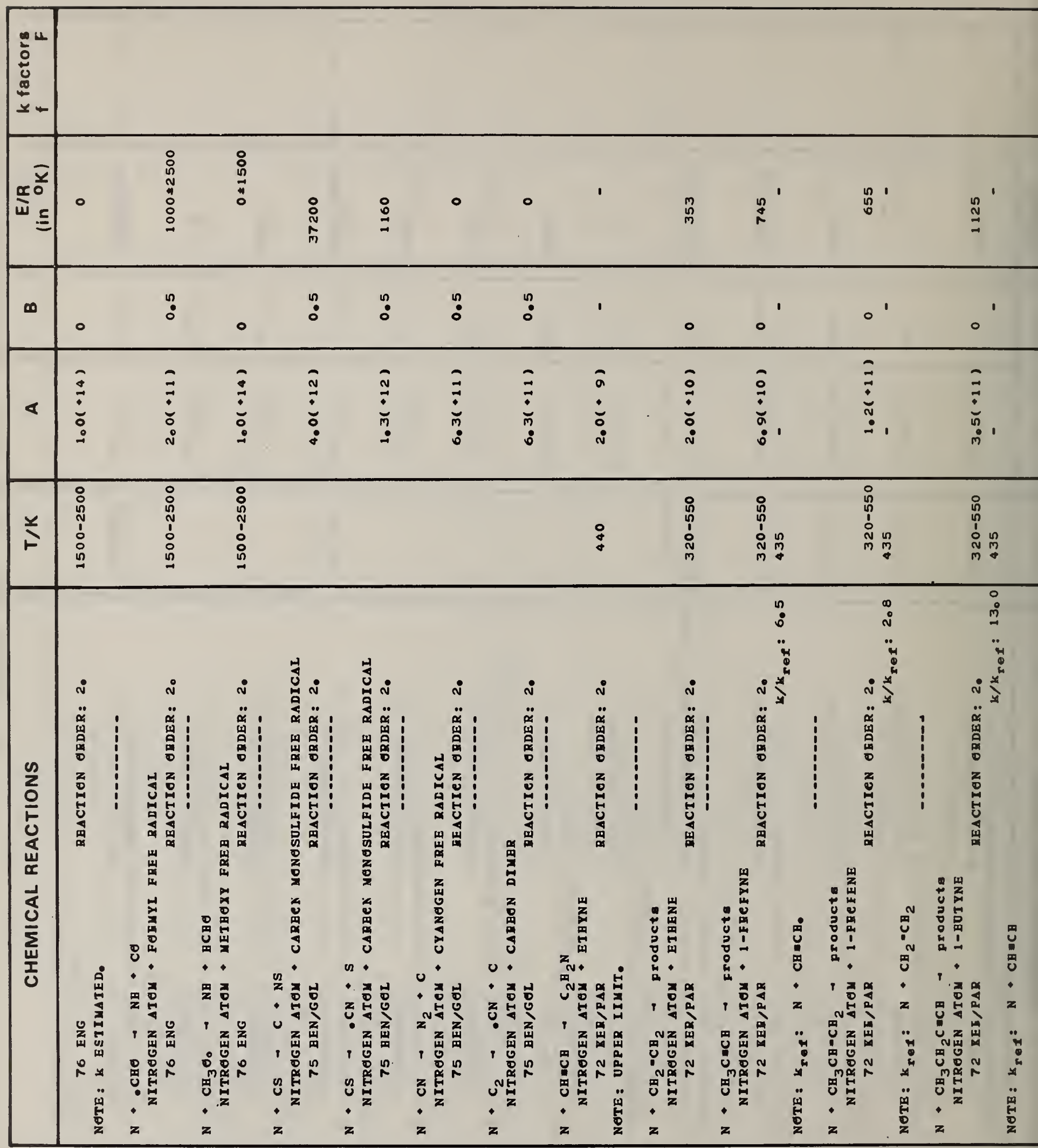




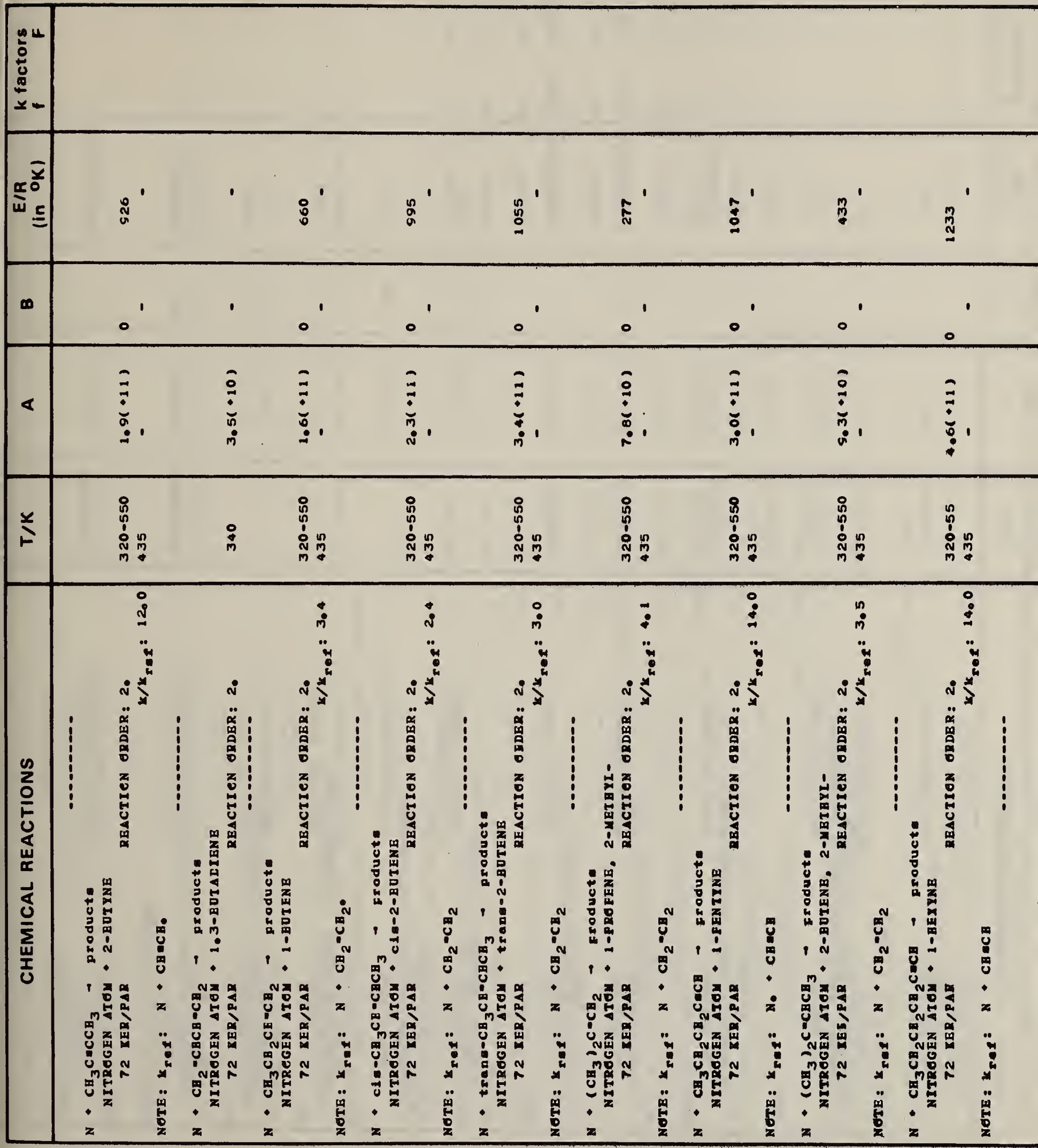




\begin{tabular}{|c|c|c|c|c|c|c|c|c|c|c|c|c|c|c|c|c|c|c|c|}
\hline 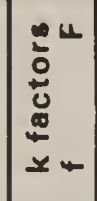 & & & & & & $\begin{array}{l}\stackrel{0}{\mathbf{i}} \\
\stackrel{n}{0} \\
\dot{0}\end{array}$ & & $\begin{array}{l}\stackrel{n}{:} \\
\stackrel{0}{0}\end{array}$ & 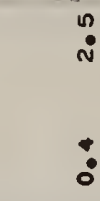 & & & & & & & & & & \\
\hline 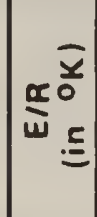 & $\stackrel{N}{ٍ}$ & & : & & & 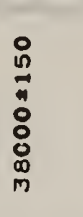 & & 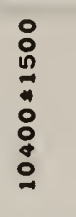 & 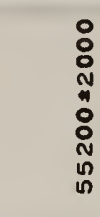 & & 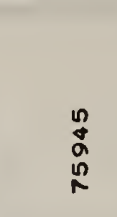 & 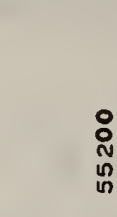 & : & 勇 & ๖̊ & 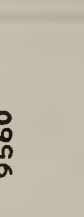 & $\begin{array}{l}: \\
\vdots \\
\vdots \\
\vdots \\
0 \\
0 \\
:\end{array}$ & & \\
\hline$\infty$ & ० & & ○ & & & 0 & & 0 & ० & & $\stackrel{n}{0}$ & $\stackrel{0}{0}$ & $\stackrel{n}{0}$ & $\stackrel{n}{0}$ & 0 & o & ० & & \\
\hline$<$ & $\begin{array}{l}\dot{\vdots} \\
\dot{m}\end{array}$ & & $\begin{array}{l}\vdots \\
\vdots \\
\vdots\end{array}$ & & & $\begin{array}{l}\frac{\hat{m}}{\grave{b}} \\
\vdots \\
\dot{0}\end{array}$ & & $\stackrel{\bar{m}}{\grave{\vdots}}$ & $\stackrel{\tilde{m}}{:}$ & & $\begin{array}{l}\tilde{m} \\
\dot{\vdots} \\
\dot{0}\end{array}$ & $\begin{array}{l}\bar{y} \\
\vdots \\
\vdots\end{array}$ & $\stackrel{\Xi}{\vdots}$ & 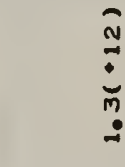 & $\begin{array}{l}\bar{\vdots} \\
\vdots \\
\vdots\end{array}$ & 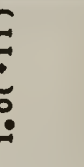 & $\stackrel{-}{\stackrel{N}{*}}$ & & 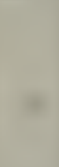 \\
\hline$\stackrel{y}{F}$ & 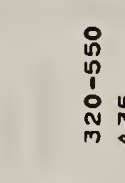 & & & & & 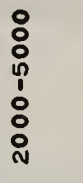 & & 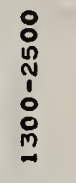 & 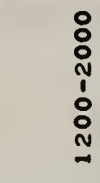 & & & & & & 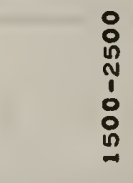 & : & $\begin{array}{l}\text { : } \\
\stackrel{\leftrightarrow}{N} \\
1 \\
\vdots \\
0\end{array}$ & & \\
\hline 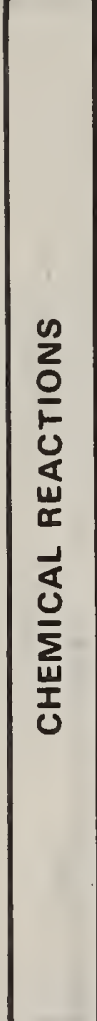 & 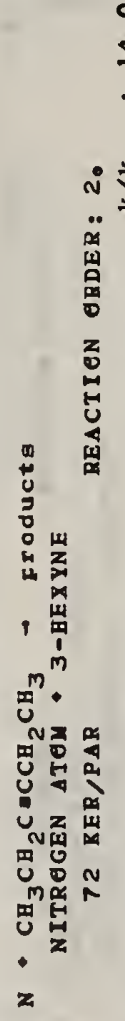 & 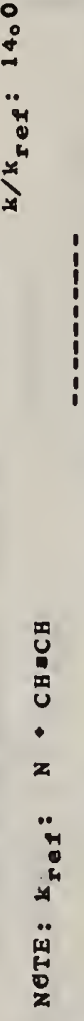 & 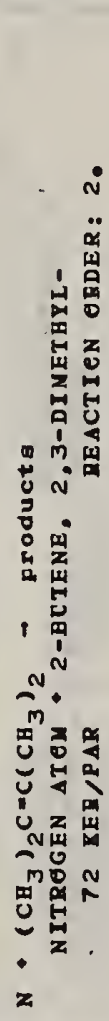 & 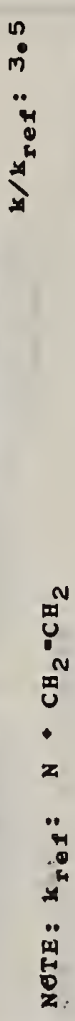 & 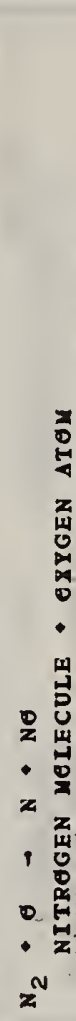 & 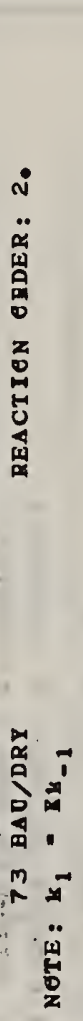 & 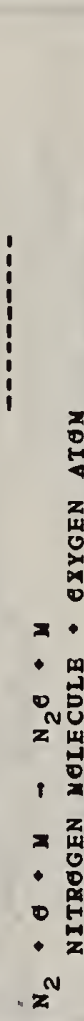 & 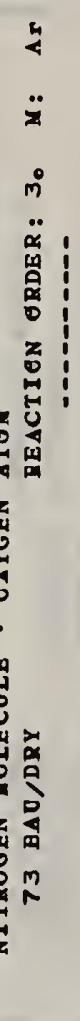 & 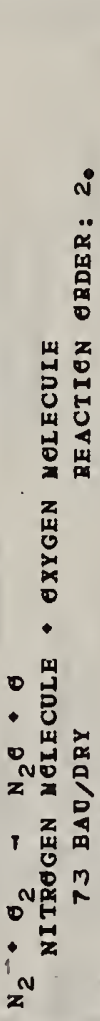 & & 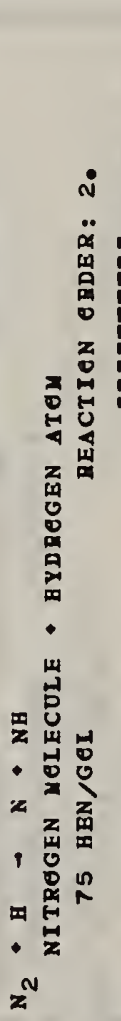 & 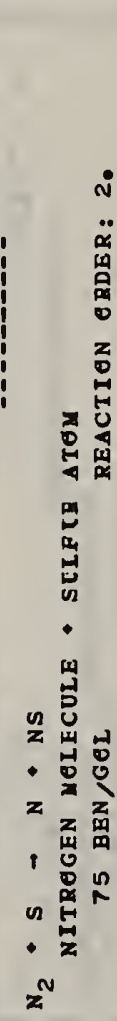 & 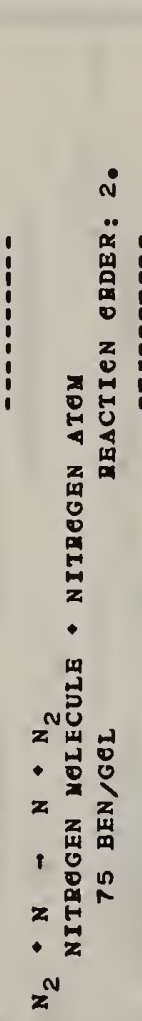 & 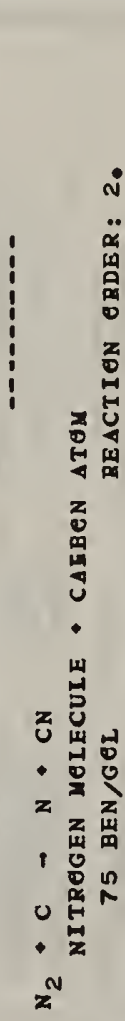 & 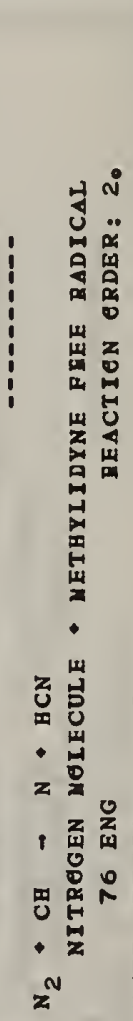 & 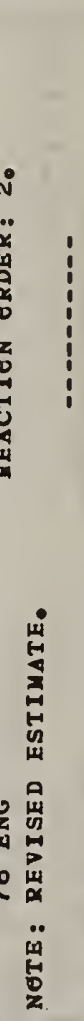 & 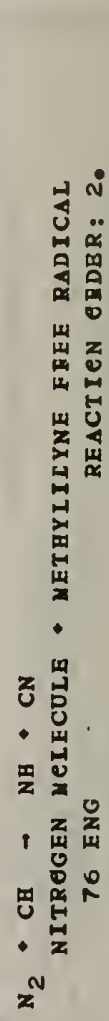 & 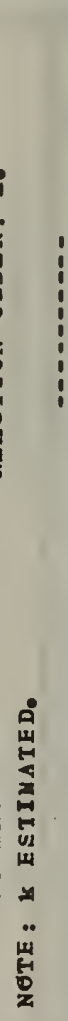 & 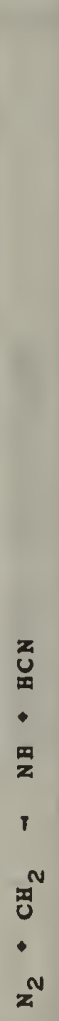 \\
\hline
\end{tabular}




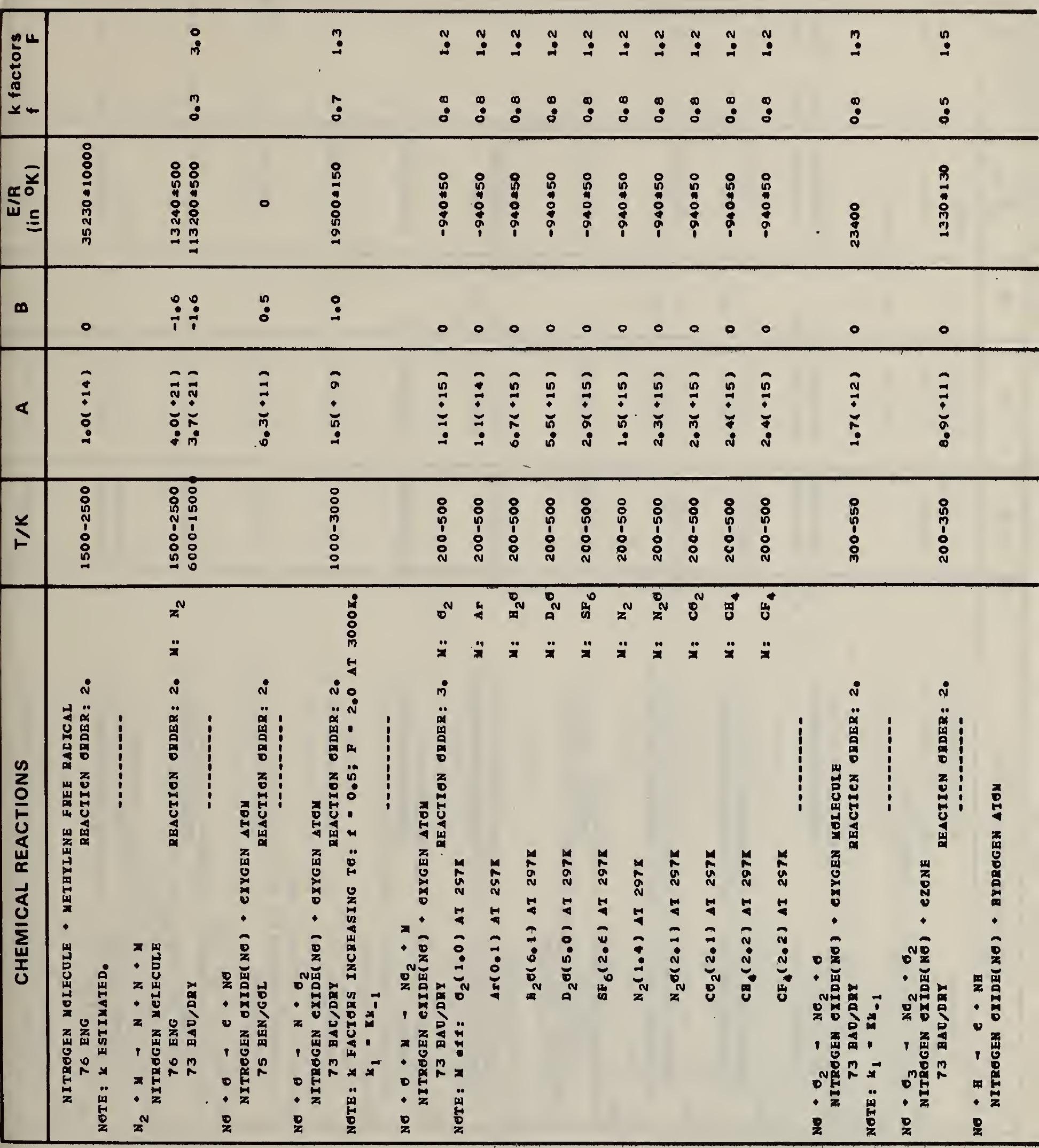




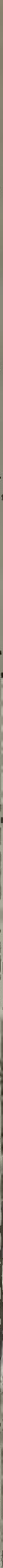




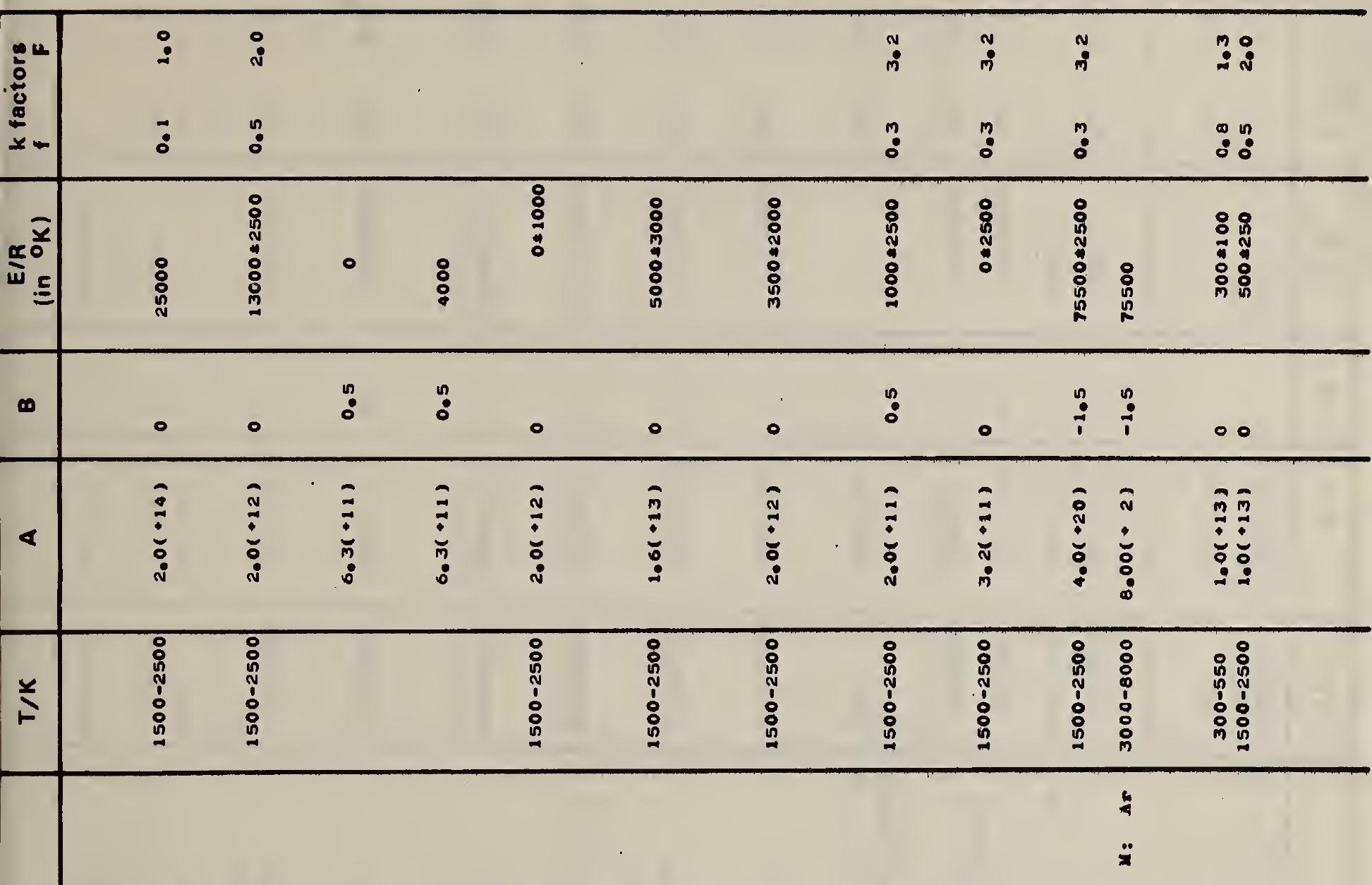

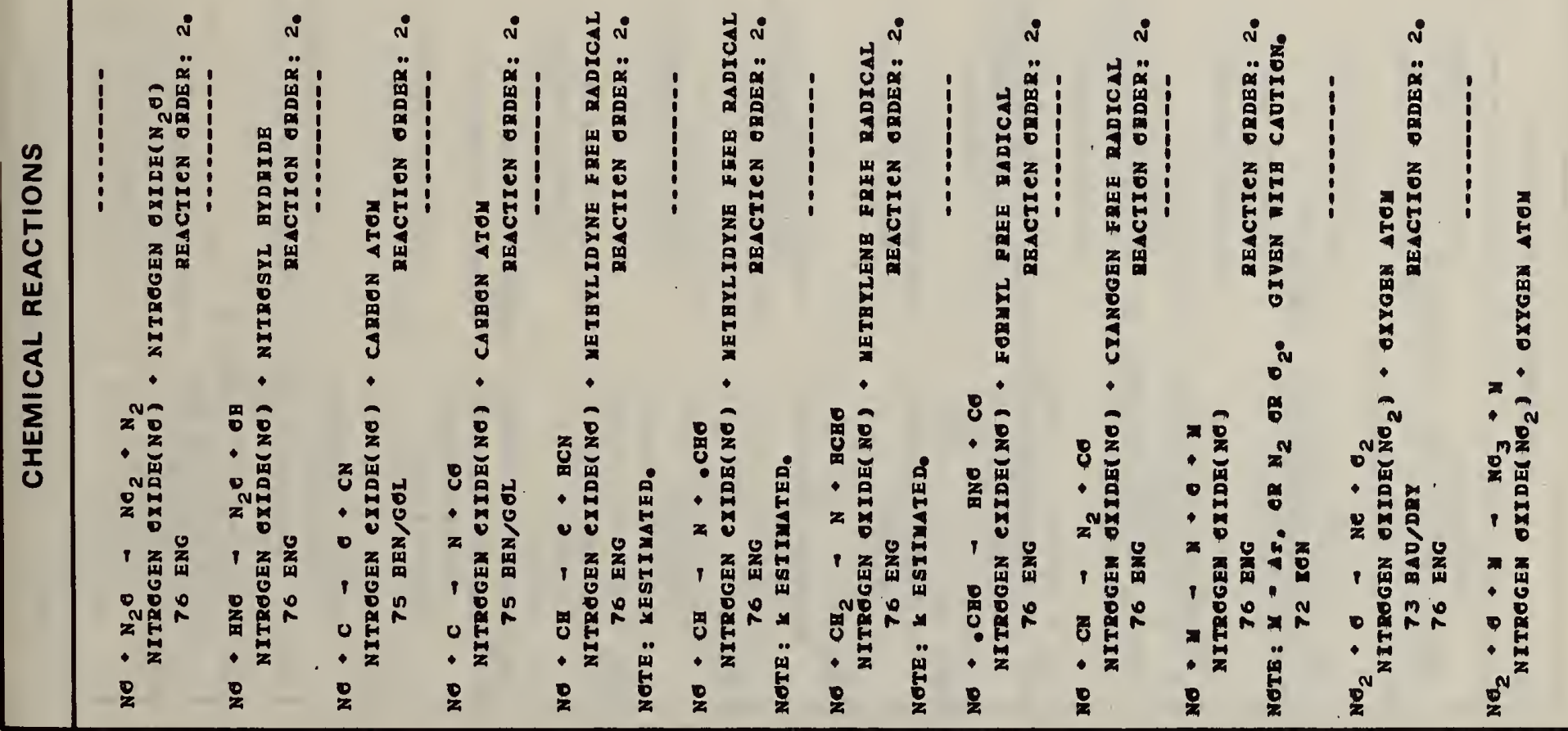




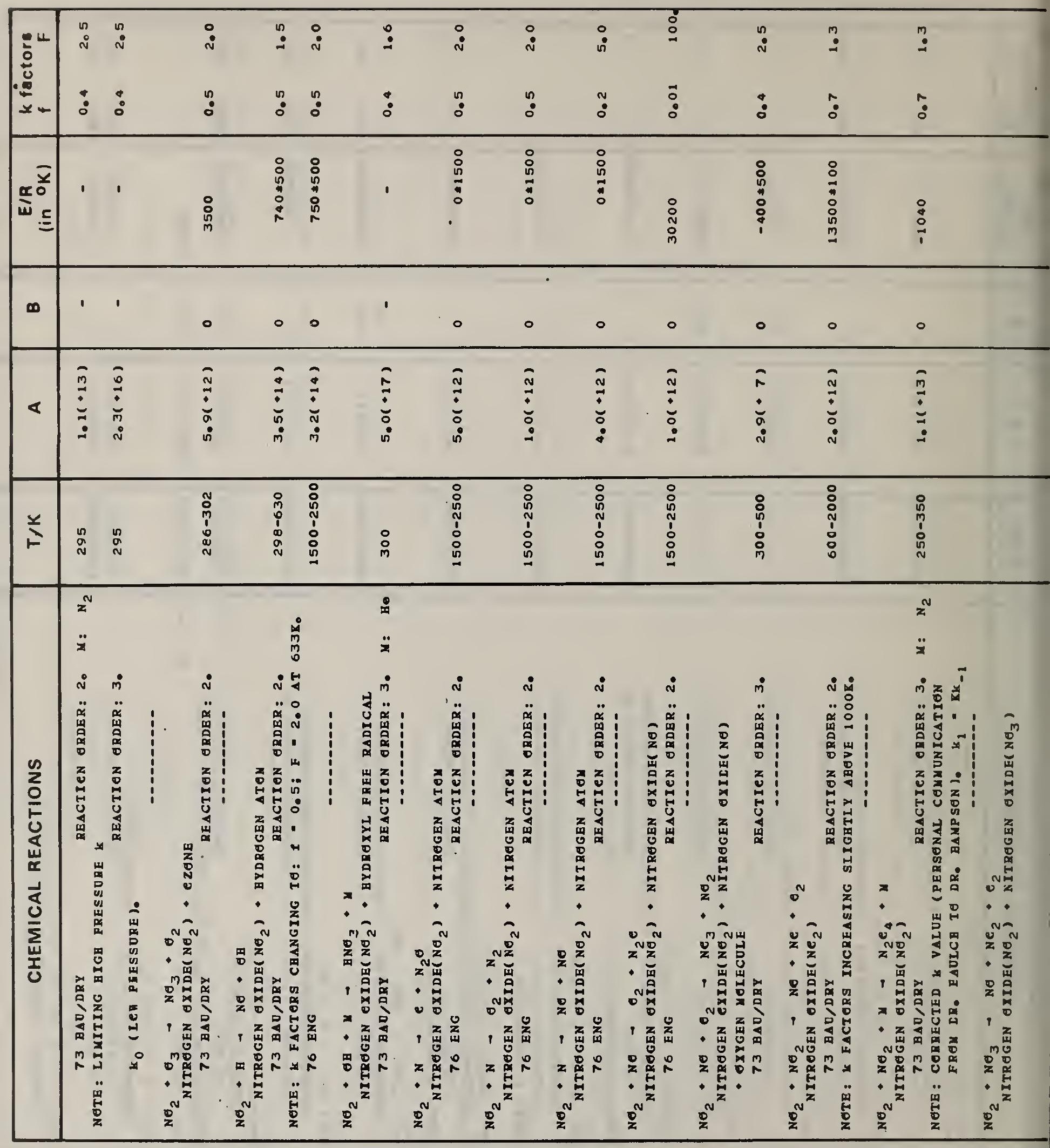




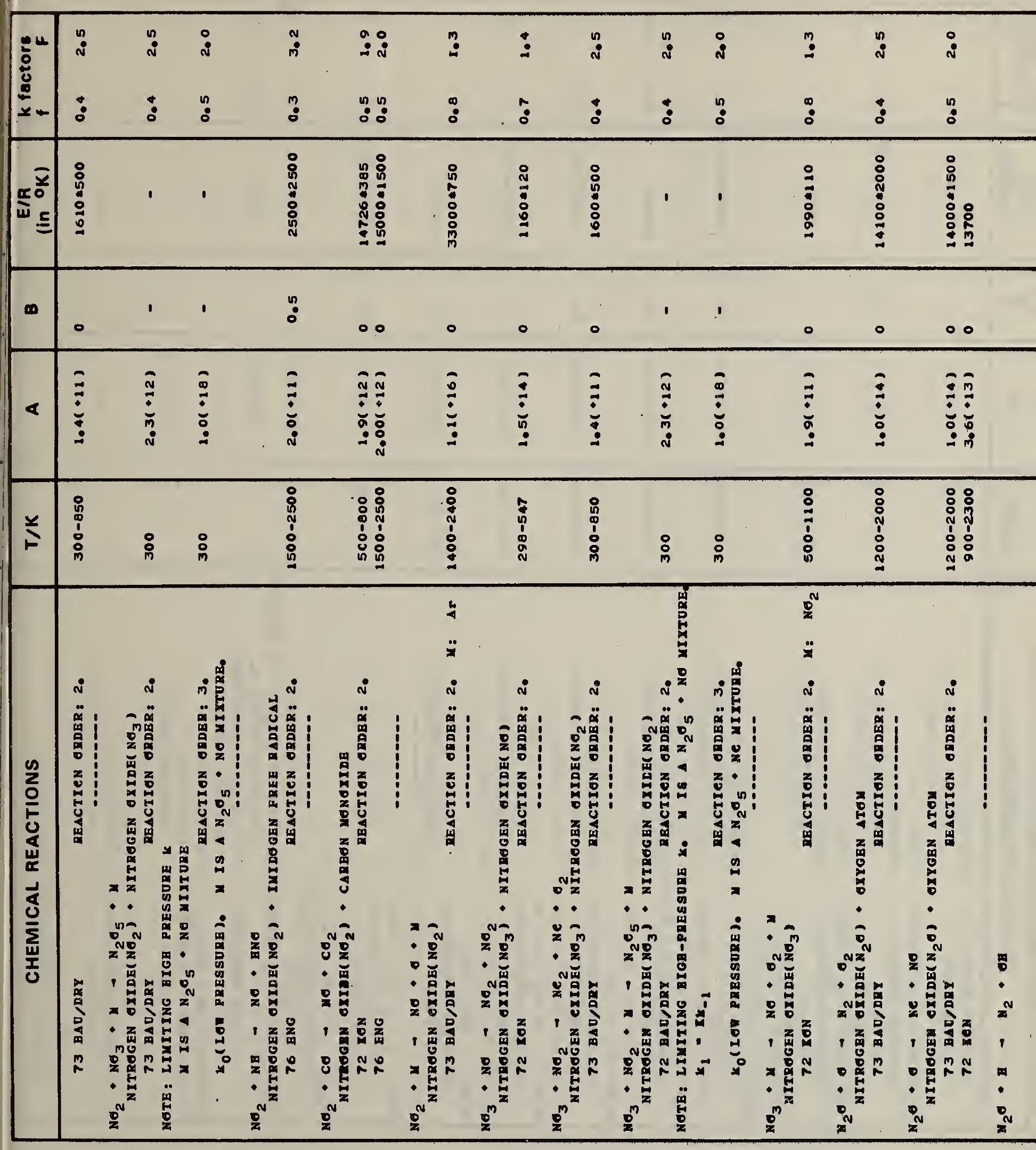




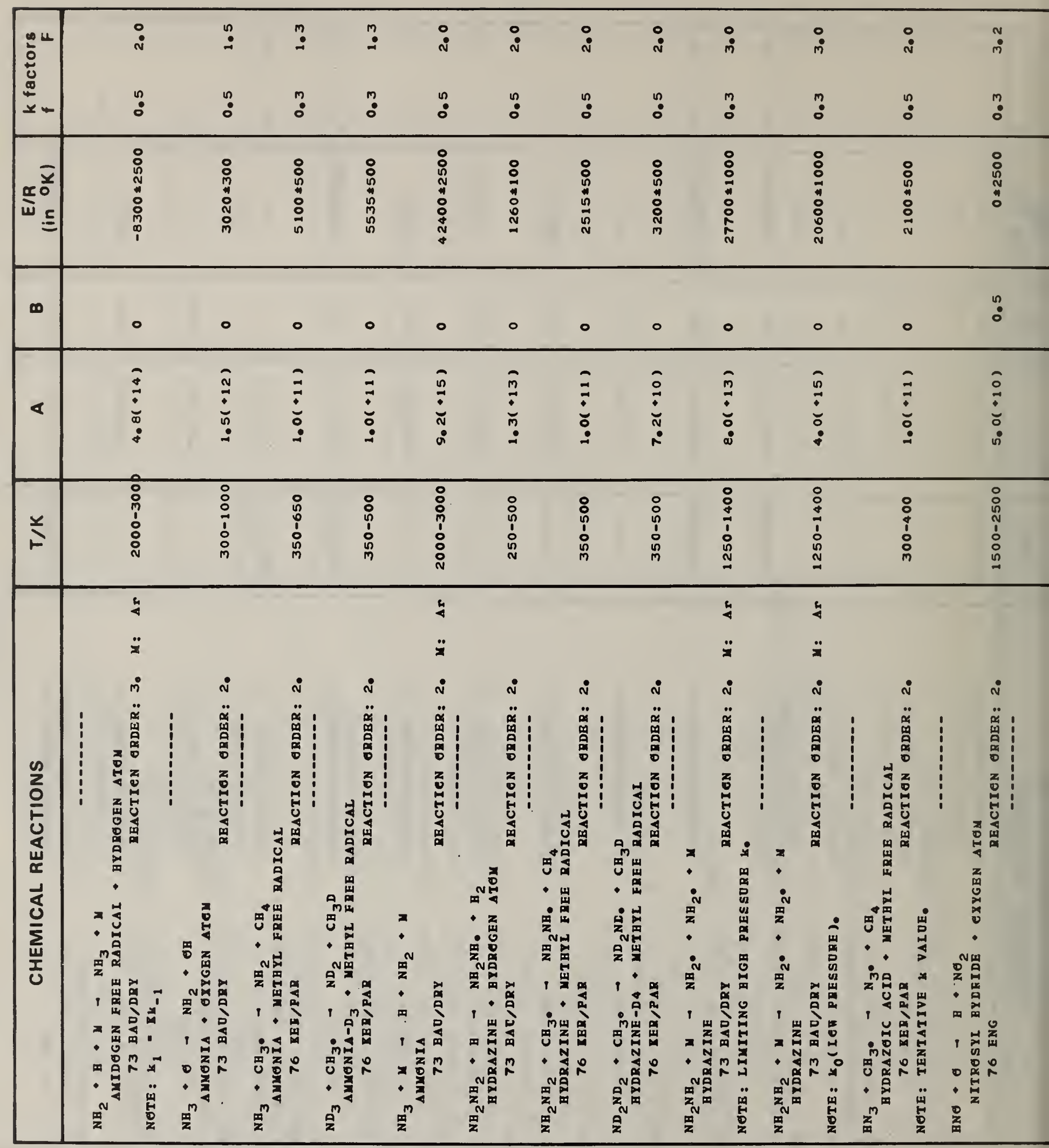




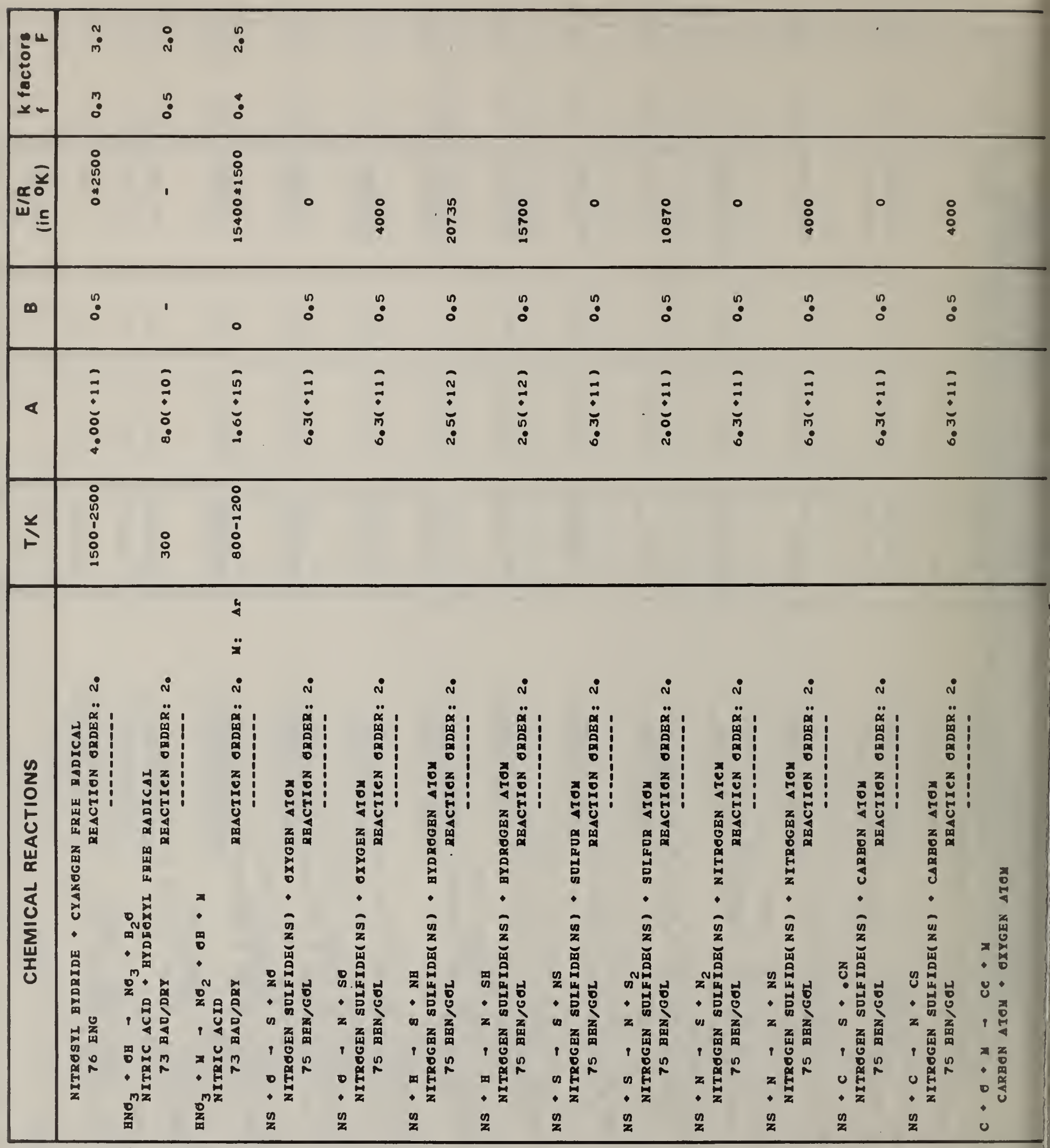




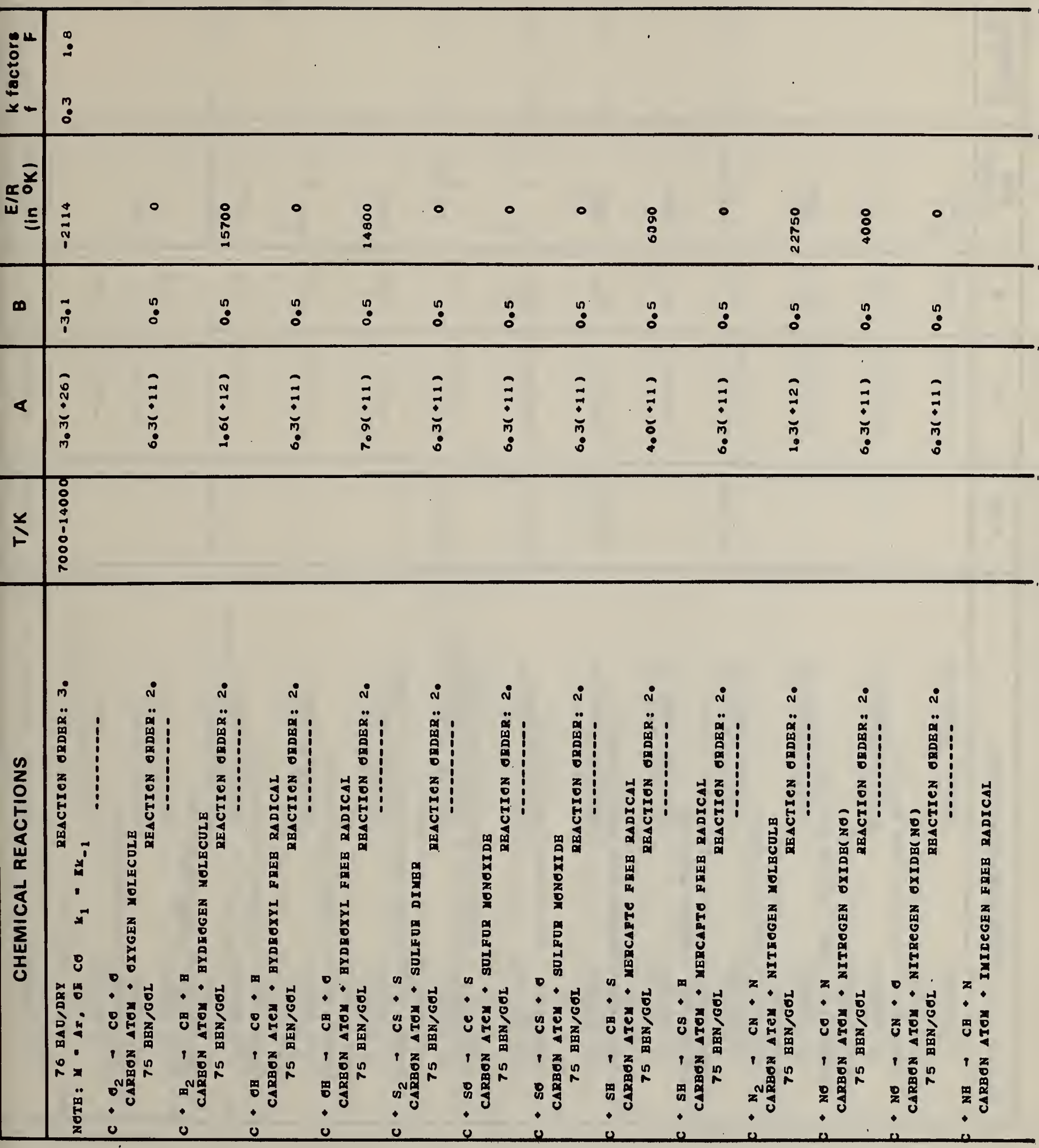




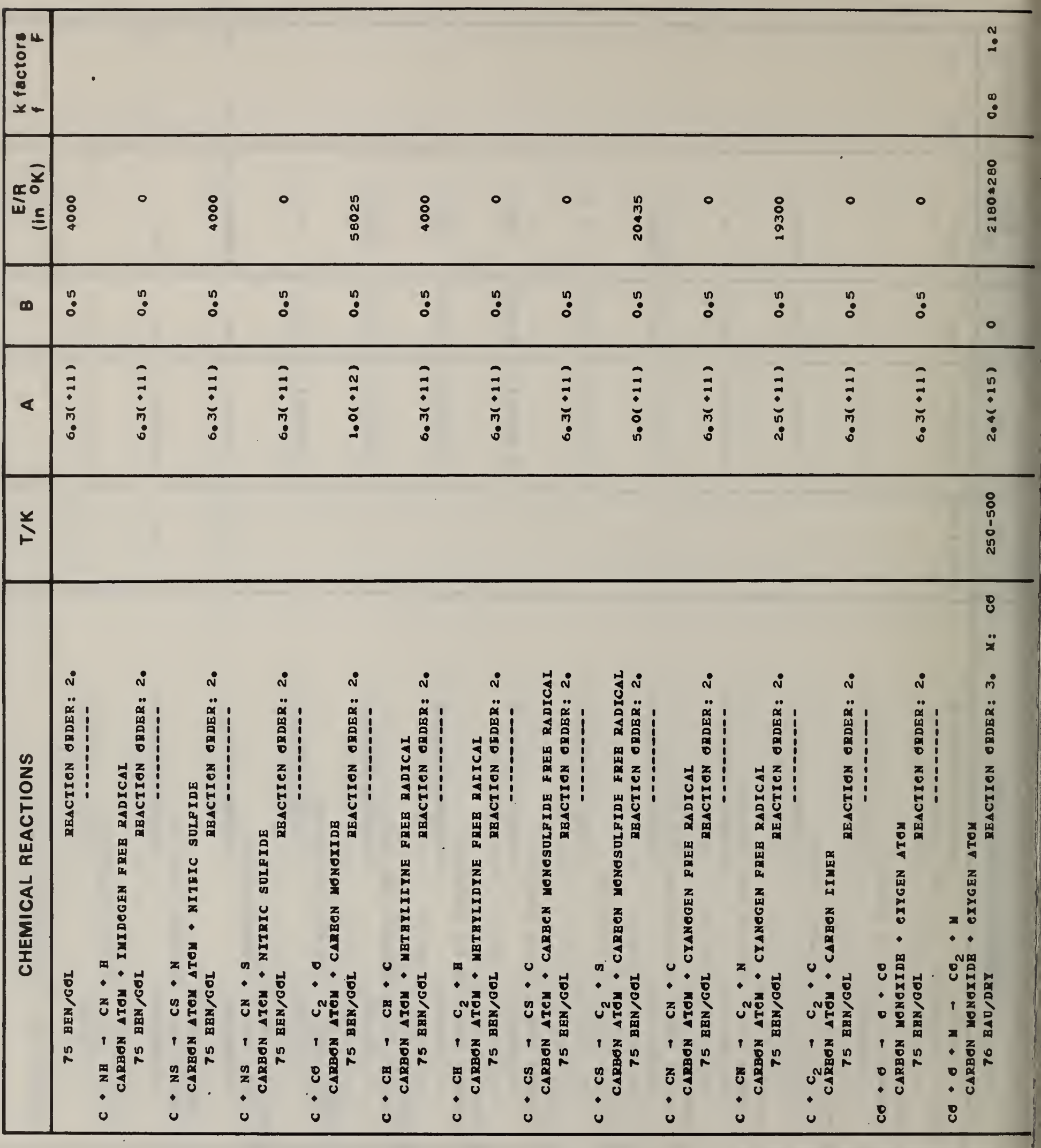




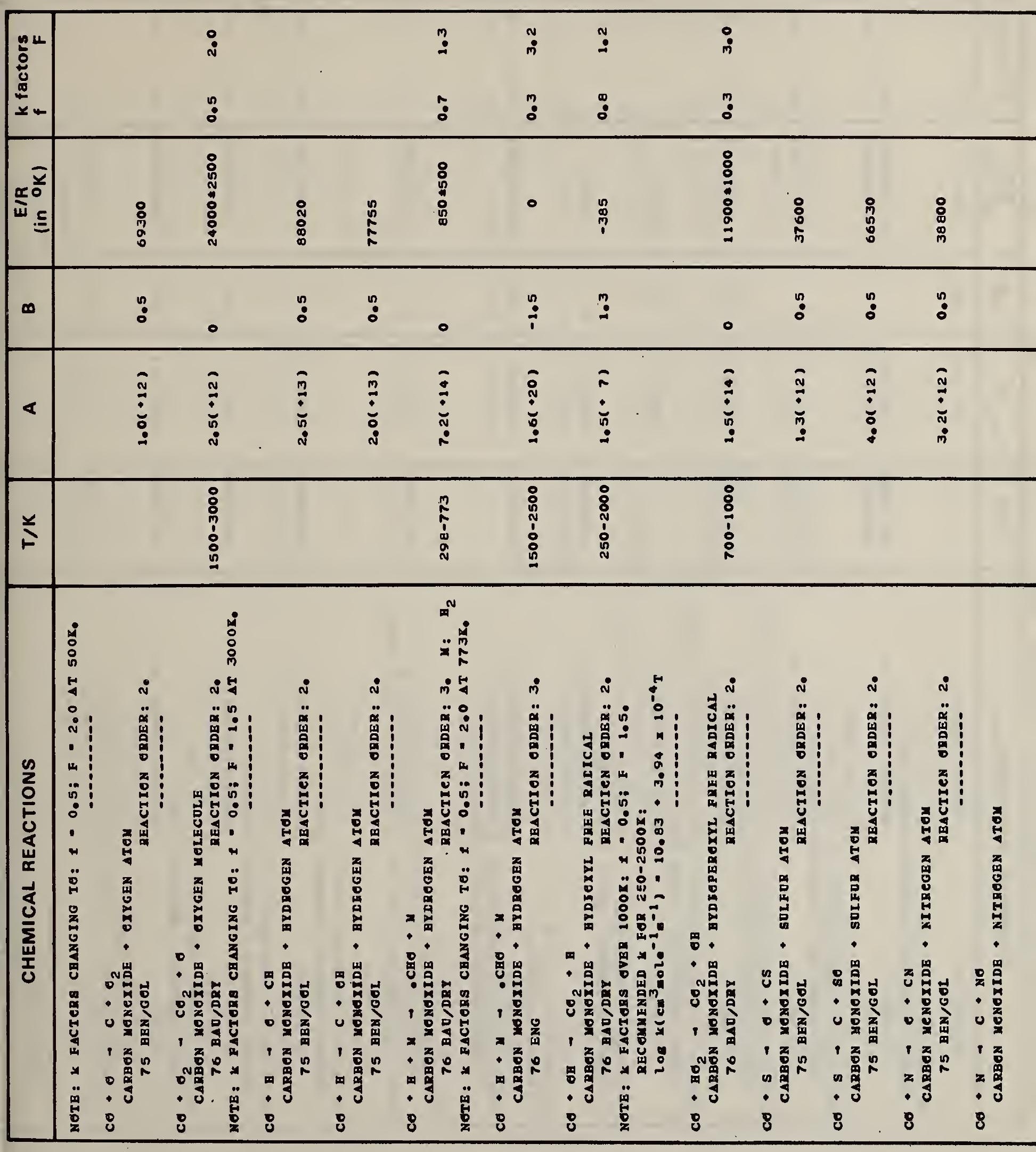




\begin{tabular}{|c|c|c|c|c|c|c|c|c|c|c|c|c|c|c|c|}
\hline 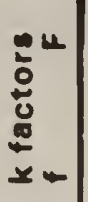 & & $\begin{array}{l}\because: \\
\vdots \text { : } \\
\because: 0\end{array}$ & $\begin{array}{l}: \\
\text { i } \\
?\end{array}$ & $\begin{array}{l}\stackrel{n}{m} \\
\dot{m}\end{array}$ & & & & $\begin{array}{l}\infty \\
: \\
\stackrel{\square}{:} \\
:\end{array}$ & $\begin{array}{l}: \\
\text { : } \\
:\end{array}$ & & $\begin{array}{l}: \\
: \\
:\end{array}$ & $\begin{array}{l}\text { m } \\
m \\
m\end{array}$ & $\begin{array}{l}: \\
:\end{array}$ & $\begin{array}{l}\stackrel{n}{m} \\
\stackrel{m}{\circ}\end{array}$ & \\
\hline 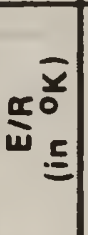 & $\stackrel{\circ}{:}$ & 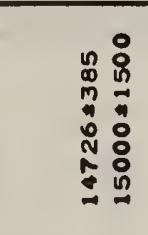 & $\begin{array}{l}: \\
: \\
: \\
\vdots \\
\vdots \\
\vdots\end{array}$ & 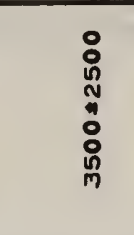 & 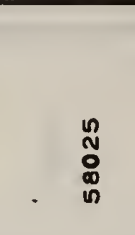 & $\stackrel{n}{\circ}$ & & 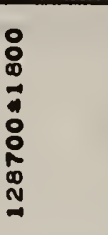 & 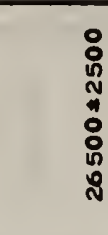 & 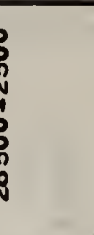 & 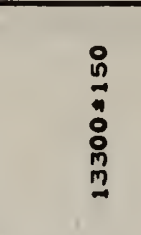 & 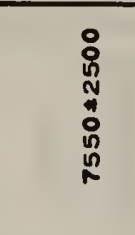 & $\begin{array}{l}: \\
: \\
\vdots \\
: \\
: \\
\vdots\end{array}$ & $\begin{array}{l}: \\
\stackrel{్}{N} \\
\text { : } \\
: \\
:\end{array}$ & \\
\hline$\infty$ & $\because$ & $\circ \circ$ & - & $\stackrel{n}{:}$ & : & 0 & & $\stackrel{?}{\dddot{m}}$ & ○ & P & - & $\stackrel{n}{0}$ & : & : & \\
\hline$<$ & $\begin{array}{l}\tilde{N} \\
\dot{\square} \\
\dot{0}\end{array}$ & 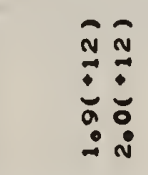 & $\begin{array}{l}\vdots \\
\vdots \\
\vdots\end{array}$ & $\stackrel{\vdots}{\vdots}$ & $\begin{array}{l}\tilde{N} \\
\vdots \\
\vdots\end{array}$ & $\begin{array}{l}\dot{\infty} \\
\dot{\Phi} \\
\dot{m}\end{array}$ & & 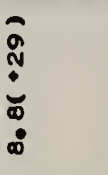 & $\begin{array}{l}n \\
\vdots \\
\vdots\end{array}$ & ? & $\begin{array}{l}\vdots \\
\vdots \\
\vdots\end{array}$ & $\begin{array}{l}a \\
\dot{0} \\
\dot{0}\end{array}$ & $\begin{array}{l}\vdots \\
\vdots \\
\dot{0}\end{array}$ & $\begin{array}{l}\hat{0} \\
\dot{:} \\
\dot{0}\end{array}$ & \\
\hline$\stackrel{Y}{上}$ & & $\begin{array}{l}\circ: \\
: \\
: \\
0 \\
1 \\
1 \\
\vdots \\
\vdots \\
: \\
0\end{array}$ & $\begin{array}{l}: \\
: \\
\stackrel{0}{n} \\
\vdots \\
\vdots \\
0\end{array}$ & $\begin{array}{l}\text { : } \\
\stackrel{\leftrightarrow}{n} \\
\vdots \\
\vdots \\
:\end{array}$ & & $\begin{array}{l}: \\
\vdots \\
\vdots \\
\stackrel{m}{n} \\
\text { N }\end{array}$ & & $\begin{array}{l}: \\
: \\
\vdots \\
\vdots \\
\vdots \\
\vdots\end{array}$ & $\begin{array}{l}: \\
: \\
\stackrel{0}{0} \\
\vdots \\
\vdots \\
\vdots\end{array}$ & 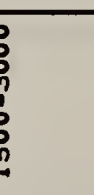 & $\begin{array}{l}: \\
: \\
i \\
\vdots \\
\vdots \\
\vdots\end{array}$ & $\begin{array}{l}\stackrel{\circ}{0} \\
\stackrel{0}{1} \\
\vdots \\
\vdots \\
0 \\
0\end{array}$ & $\begin{array}{l}: \\
: \\
\stackrel{D}{0} \\
\vdots \\
\vdots \\
\vdots \\
0\end{array}$ & $\begin{array}{l}: \\
: \\
\stackrel{0}{0} \\
\vdots \\
\vdots \\
0\end{array}$ & \\
\hline 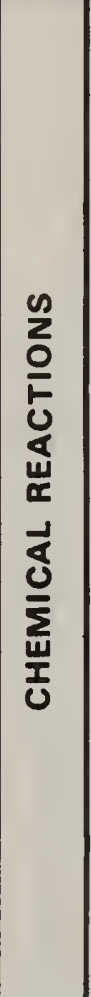 & 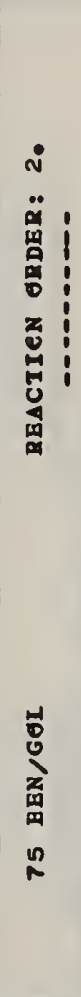 & 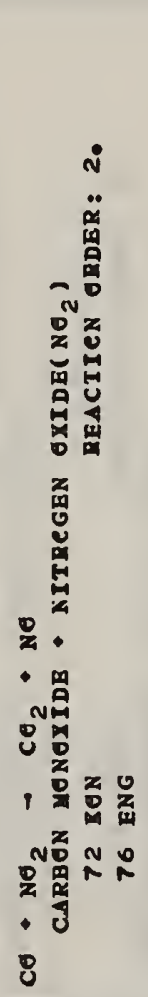 & 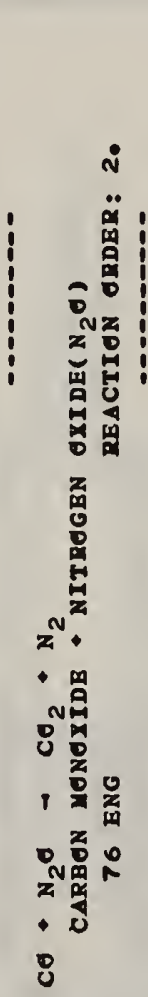 & 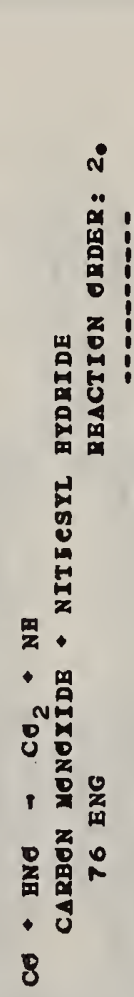 & 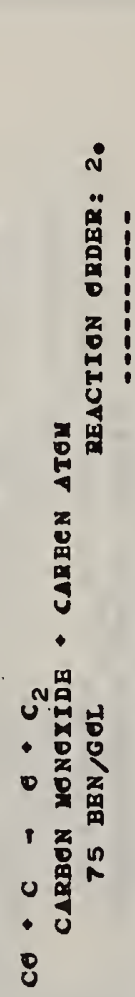 & 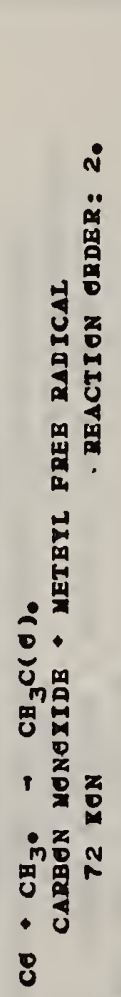 & $\begin{array}{l}\text { D } \\
\end{array}$ & 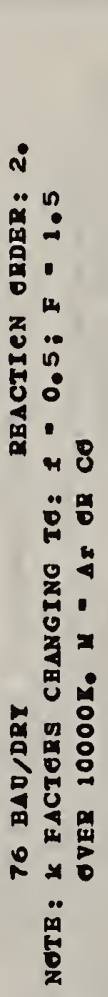 & 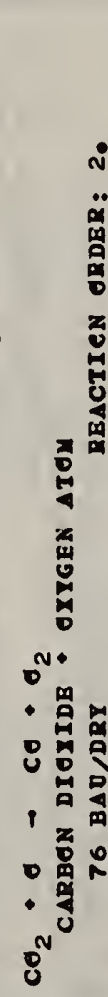 & 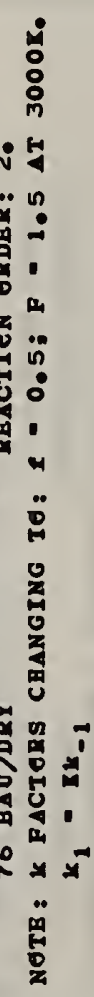 & 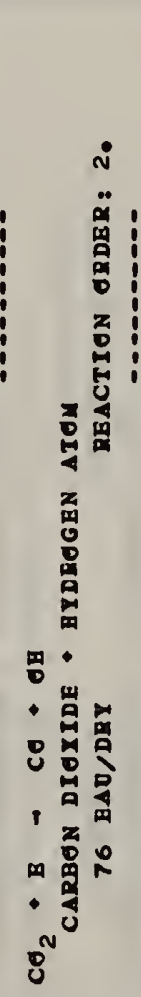 & 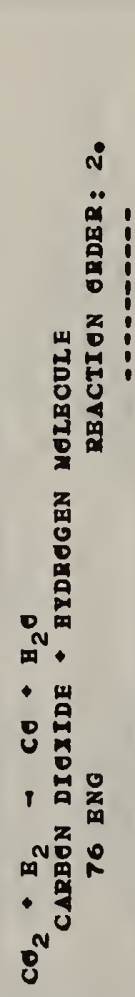 & 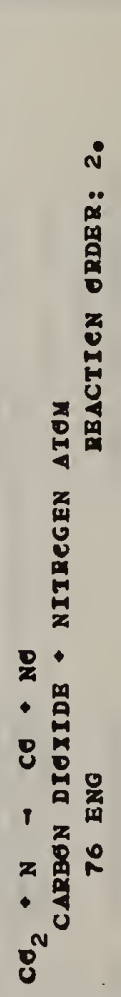 & 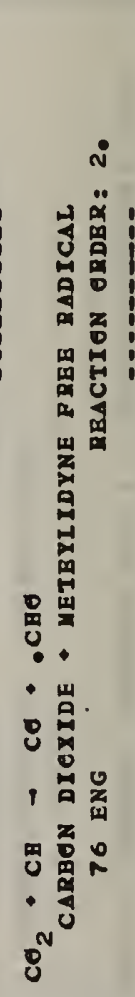 & 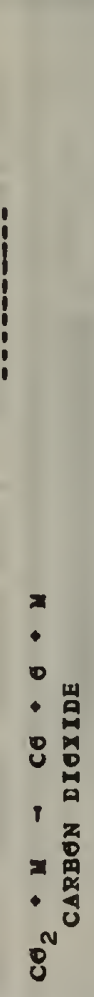 \\
\hline
\end{tabular}




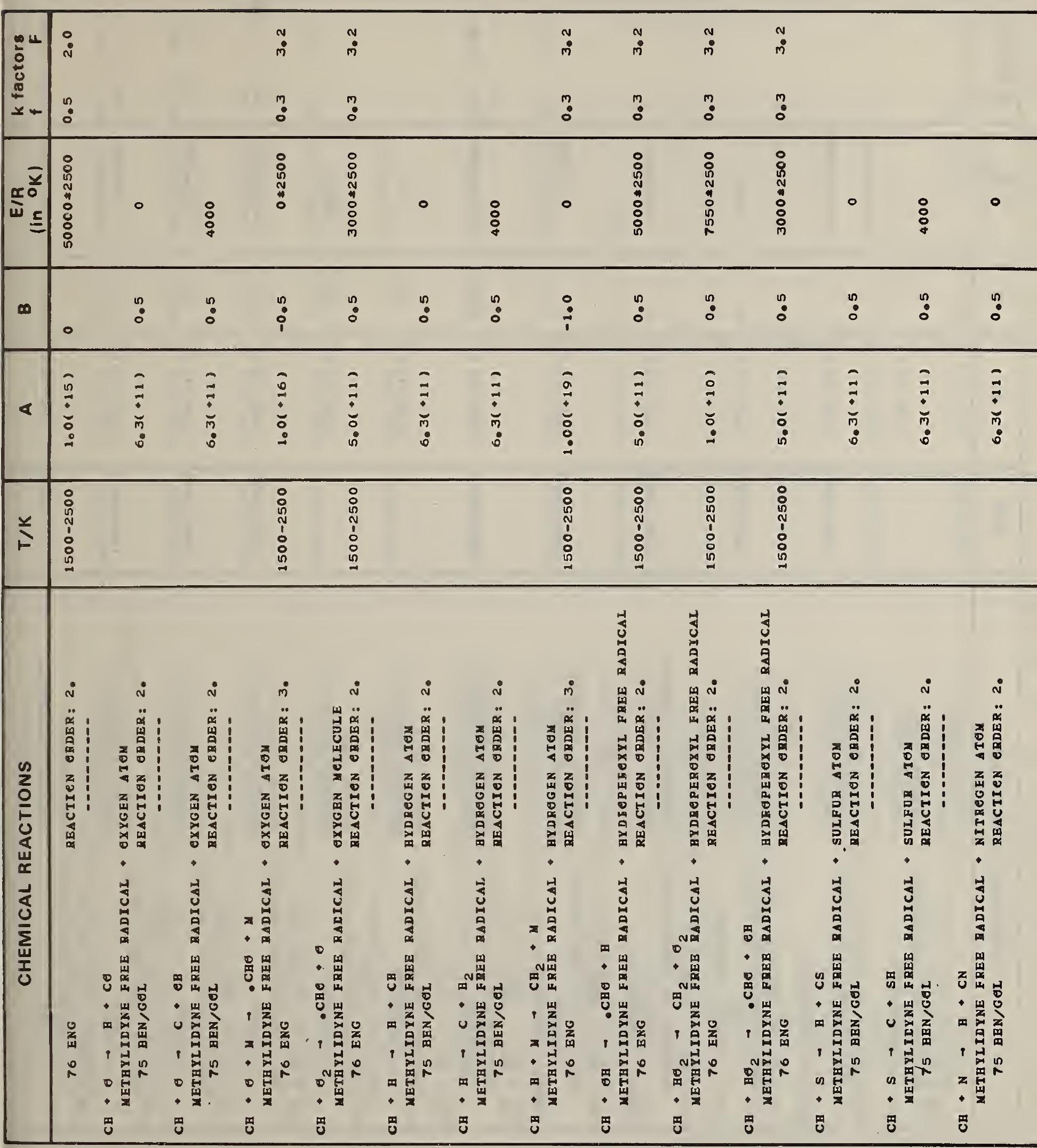




\begin{tabular}{|c|c|c|c|c|c|c|c|c|c|c|c|c|}
\hline 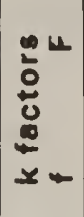 & & & & & & $\begin{array}{l}\stackrel{N}{\circ} \\
\dot{m} \\
\stackrel{m}{\circ}\end{array}$ & & & $\begin{array}{l}\stackrel{N}{\dot{m}} \\
\stackrel{m}{\circ}\end{array}$ & $\begin{array}{l}\stackrel{n}{\dot{m}} \\
\stackrel{m}{\circ}\end{array}$ & $\begin{array}{l}\stackrel{n}{\circ} \\
\stackrel{m}{\circ}\end{array}$ & 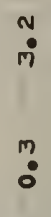 \\
\hline $\begin{array}{l}\frac{y}{0} \\
\bar{\omega}\end{array}$ & $\begin{array}{l}n \\
0 \\
0 \\
0 \\
0\end{array}$ & $\begin{array}{l}: \\
\vdots \\
\vdots \\
\vdots \\
\vdots \\
0 \\
0 \\
0\end{array}$ & $\begin{array}{l}: \\
: \\
\vdots \\
0\end{array}$ & $\begin{array}{l}: \\
: \\
: \\
⿱ 艹 \\
: \\
:\end{array}$ & 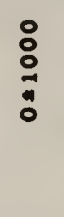 & 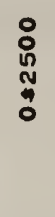 & 0 & $:$ & 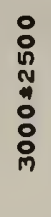 & 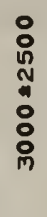 & 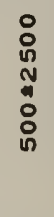 & $\begin{array}{l}\text { : } \\
\stackrel{d}{0} \\
\vdots \\
\vdots \\
0\end{array}$ \\
\hline$\infty$ & $\stackrel{n}{0}$ & 0 & ० & 0 & 0 & $\stackrel{n}{0}$ & $\stackrel{n}{0}$ & $\stackrel{!}{0}$ & $\stackrel{n}{0}$ & : & : & $\grave{0}$ \\
\hline$<$ & $\begin{array}{l}\tilde{N} \\
\vdots \\
\end{array}$ & 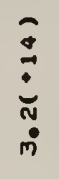 & $\begin{array}{l}\vdots \\
\vdots \\
\vdots\end{array}$ & $\stackrel{n}{m}$ & $\begin{array}{l}\mathfrak{y} \\
\vdots \\
\dot{\vdots}\end{array}$ & $\bar{\Xi}$ & $\bar{\Xi}$ & $\bar{\Xi}$ & & $\begin{array}{l}\vdots \\
\vdots \\
\dot{n} \\
\dot{n}\end{array}$ & $\begin{array}{l}\hat{0} \\
\dot{5} \\
\dot{0} \\
\dot{m}\end{array}$ & $\vdots$ \\
\hline 兰 & & 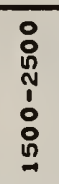 & 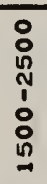 & $\begin{array}{l} \\
\\
: \\
1 \\
\vdots \\
\vdots \\
0 \\
0\end{array}$ & 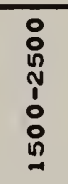 & 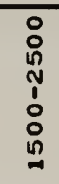 & & & 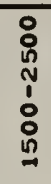 & 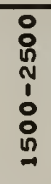 & 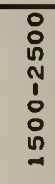 & 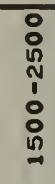 \\
\hline
\end{tabular}

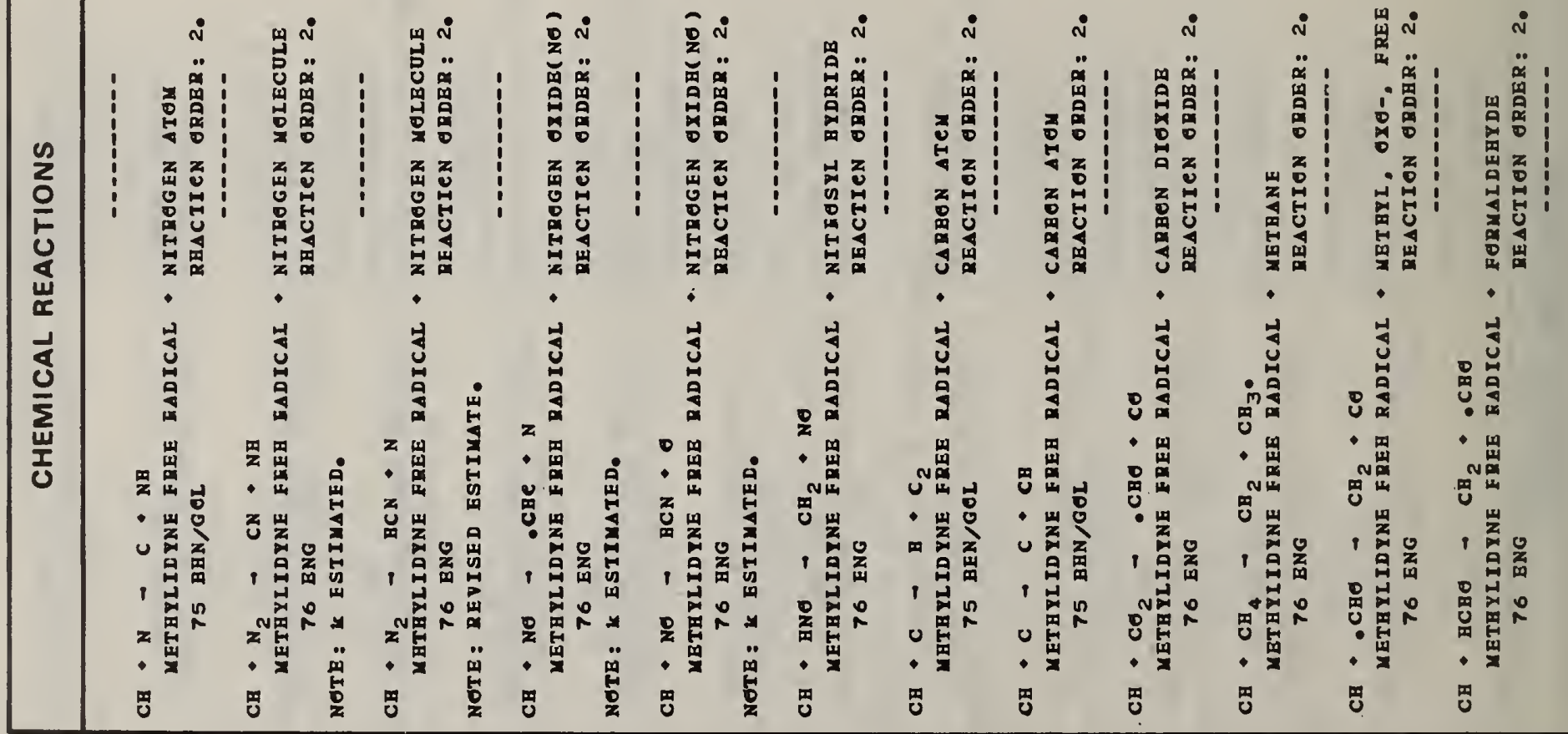




\begin{tabular}{|c|c|c|c|c|c|c|c|c|c|c|c|c|}
\hline 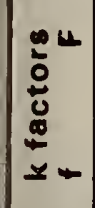 & $\begin{array}{l}\stackrel{N}{\dot{m}} \\
\stackrel{m}{0}\end{array}$ & $\begin{array}{l}\stackrel{N}{m} \\
:\end{array}$ & $\begin{array}{l}\stackrel{N}{0} \\
\ddot{m} \\
\stackrel{m}{0}\end{array}$ & $\begin{array}{l}\stackrel{N}{m} \\
\dot{m} \\
\dot{0} \\
\ddot{0}\end{array}$ & & $\begin{array}{l}\text { No } \\
\dot{m} \\
\dot{m} \\
\stackrel{0}{0}\end{array}$ & $\begin{array}{l}\text { No } \\
\dot{m} \\
\stackrel{m}{0}\end{array}$ & & $\begin{array}{l}\stackrel{N}{m} \\
\dot{m} \\
\ddot{m}\end{array}$ & & & : \\
\hline 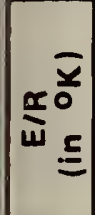 & 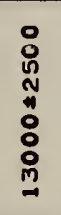 & $\begin{array}{l}: \\
: \\
\stackrel{H}{N} \\
: \\
: \\
: \\
\stackrel{D}{0}\end{array}$ & 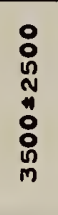 & 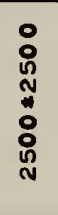 & $\begin{array}{l}\text { : } \\
\stackrel{n}{0} \\
\stackrel{n}{N} \\
\stackrel{n}{n}\end{array}$ & 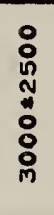 & 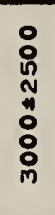 & : & 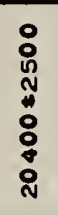 & \begin{tabular}{l}
$:$ \\
$:$ \\
$:$ \\
\hdashline \\
$\circ$ \\
$\stackrel{n}{n}$ \\
n \\
$m$
\end{tabular} & $\begin{array}{l}: \\
: \\
: \\
: \\
: \\
: \\
\text { in }\end{array}$ & $\begin{array}{l}: \\
\stackrel{\leftrightarrow}{n} \\
\mathbb{N} \\
0\end{array}$ \\
\hline$\infty$ & $\grave{:}$ & ?: & $\stackrel{?}{:}$ & 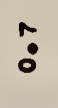 & 0 & $\stackrel{\text { ? }}{:}$ & : & - & : & ० & 0 & ! \\
\hline$<$ & 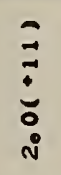 & $\begin{array}{l}\bar{\vdots} \\
\vdots \\
\end{array}$ & $\begin{array}{l}\bar{\vdots} \\
\dot{0} \\
\dot{0}\end{array}$ & $\begin{array}{l}\vdots \\
\vdots \\
\dot{n} \\
\dot{m}\end{array}$ & $\underset{\stackrel{N}{N}}{\tilde{m}}$ & $\begin{array}{l}\vdots \\
\vdots \\
\vdots\end{array}$ & $\begin{array}{l}\overline{1} \\
\vdots \\
0\end{array}$ & $\stackrel{n}{n}$ & $\bar{\Xi}$ & $\stackrel{\vdots}{\vdots}$ & 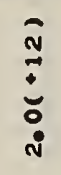 & $=$ \\
\hline$\stackrel{Y}{-}$ & $\begin{array}{l}: \\
: \\
\stackrel{n}{N} \\
\vdots \\
: \\
0\end{array}$ & $\begin{array}{l}0 \\
: \\
\stackrel{0}{N} \\
\vdots \\
\vdots \\
:\end{array}$ & $\begin{array}{l}0 \\
: \\
\stackrel{0}{N} \\
\vdots \\
\vdots \\
0 \\
0\end{array}$ & 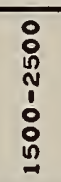 & 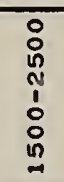 & $\begin{array}{l}: \\
: \\
0 \\
\vdots \\
\vdots \\
0\end{array}$ & $\begin{array}{l}0 \\
\vdots \\
0 \\
0 \\
\vdots \\
\vdots \\
\vdots\end{array}$ & $\begin{array}{l}: \\
: \\
\stackrel{\circ}{1} \\
\vdots \\
\vdots \\
0\end{array}$ & 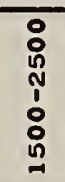 & 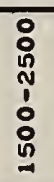 & $\begin{array}{l}: \\
: \\
0 \\
\vdots \\
\vdots \\
\vdots \\
0\end{array}$ & $\begin{array}{l}\text { : } \\
\stackrel{0}{N} \\
\vdots \\
: \\
:\end{array}$ \\
\hline
\end{tabular}

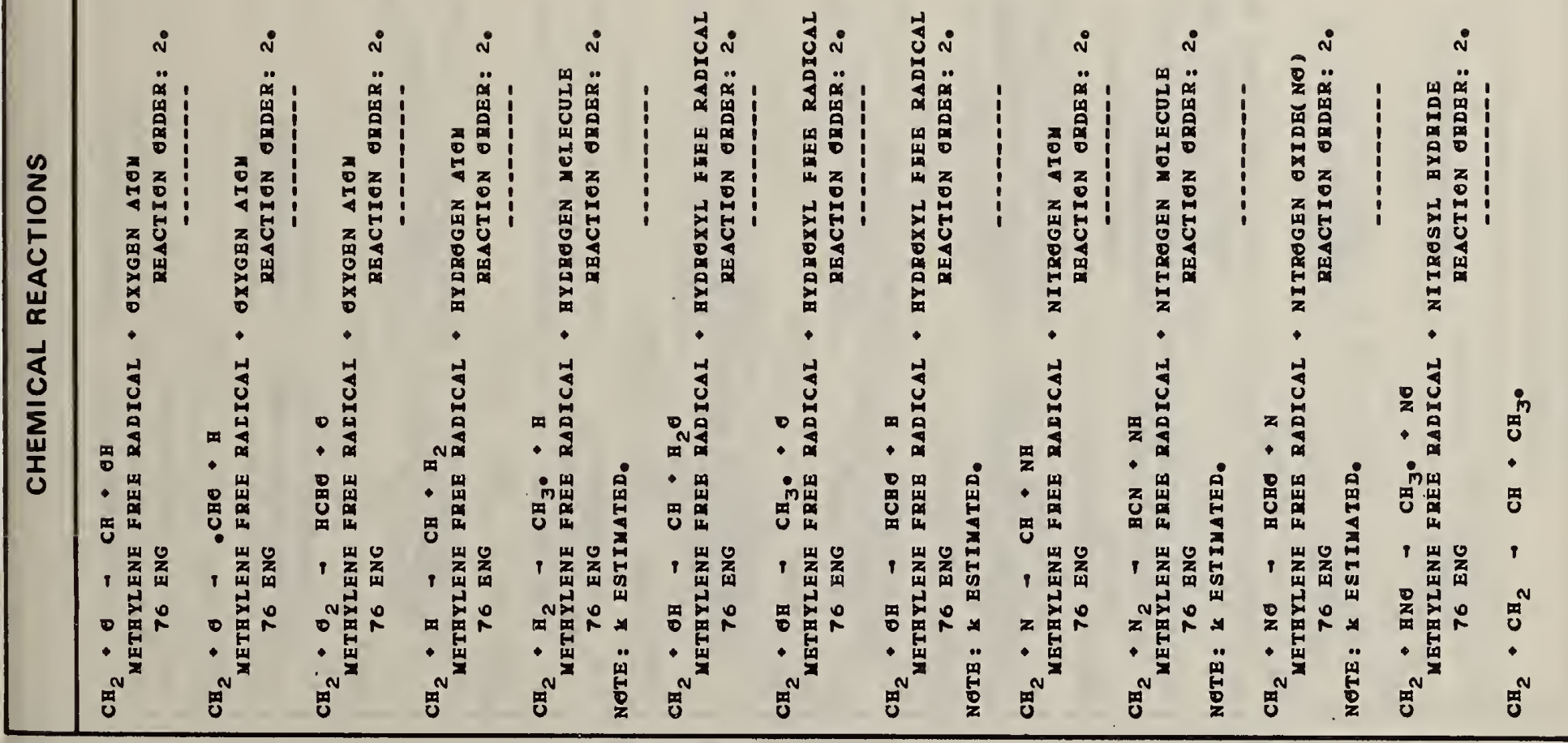




\begin{tabular}{|c|c|c|c|c|c|c|c|c|c|c|c|c|c|c|c|c|c|c|c|c|c|c|c|}
\hline 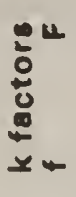 & $\begin{array}{l}\text { N } \\
m \\
m \\
m\end{array}$ & $\begin{array}{l}N \\
m \\
m\end{array}$ & $\begin{array}{l}\text { N } \\
? \\
0\end{array}$ & $\begin{array}{l}\mathfrak{N} \\
\dot{m} \\
m \\
\dot{m}\end{array}$ & & & & & & & & & & & & & & & & & " & & \\
\hline$\underbrace{\underline{x}}_{\underline{\omega}}$ & 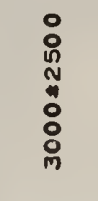 & $\begin{array}{l}\circ \\
\stackrel{8}{0} \\
\text { N } \\
: \\
: \\
:\end{array}$ & 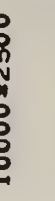 & 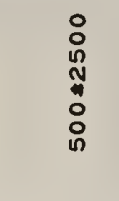 & 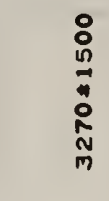 & & 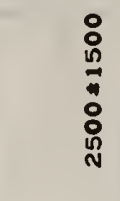 & & 1 & & & 1 & & I & & & ' & & 1 & & 1 & & \\
\hline$\infty$ & ? & $\because$ & $:$ & $\because$ & 0 & & 0 & & 1 & & & 1 & & 1 & & & 1 & & 1 & & ${ }^{\prime}$ & & \\
\hline$\alpha$ & $\begin{array}{l}\vec{E} \\
\dot{0} \\
\dot{0}\end{array}$ & $\vec{v}$ & $\vec{n}$ & $\begin{array}{l}0 \\
\stackrel{0}{*} \\
\stackrel{0}{m}\end{array}$ & $\begin{array}{l}\Xi \\
\vdots \\
\vdots\end{array}$ & & $\begin{array}{l}\vec{N} \\
\stackrel{N}{*} \\
\dot{m}\end{array}$ & & 1 & 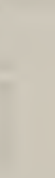 & & 1 & & 1 & & & 1 & & I & & 1 & & \\
\hline$\stackrel{Y}{F}$ & $\begin{array}{l}\circ \\
: \\
\stackrel{0}{n} \\
\vdots \\
\vdots \\
:\end{array}$ & 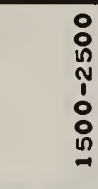 & 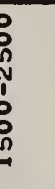 & 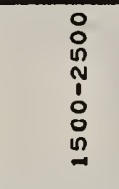 & $\begin{array}{l}\circ \\
\stackrel{0}{n} \\
N \\
\vdots \\
: \\
\stackrel{0}{n}\end{array}$ & & 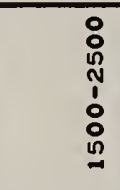 & & $\stackrel{\sim}{\alpha}$ & 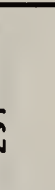 & & $\hat{\sigma}$ & & No & & & $\hat{\alpha}$ & & $\stackrel{i}{\sim}$ & & 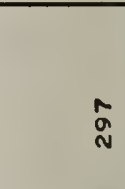 & & \\
\hline 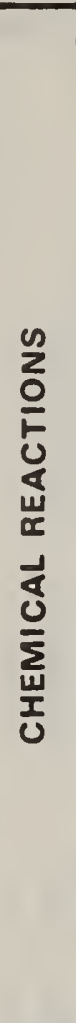 & 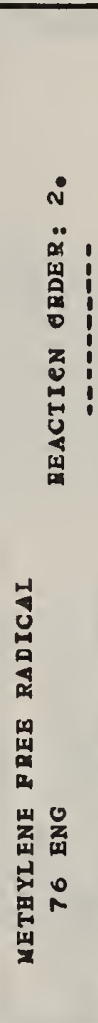 & 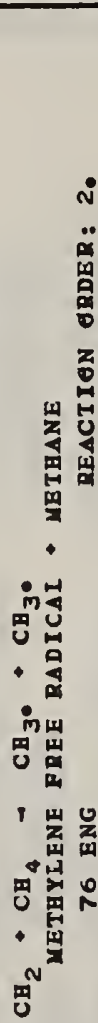 & 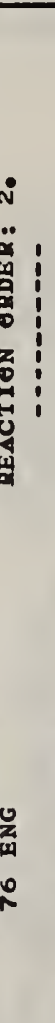 & 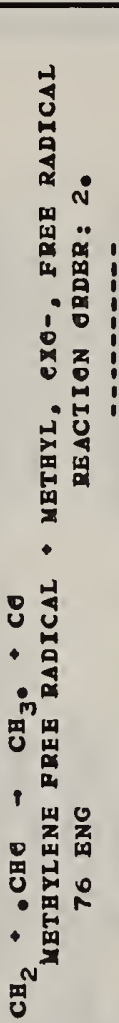 & 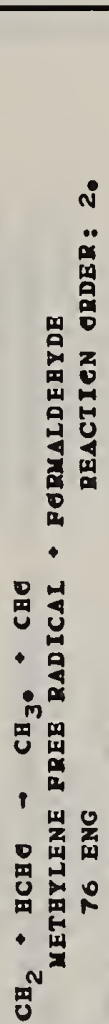 & 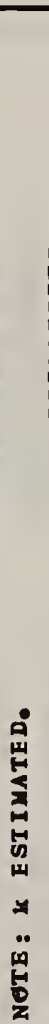 & 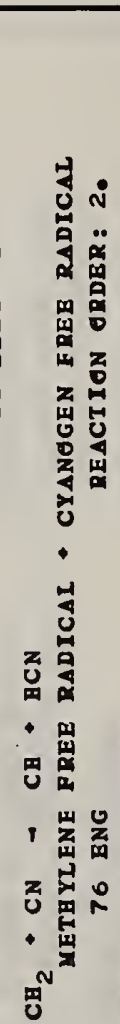 & 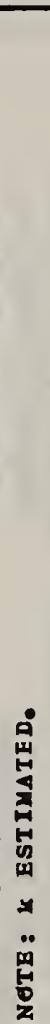 & 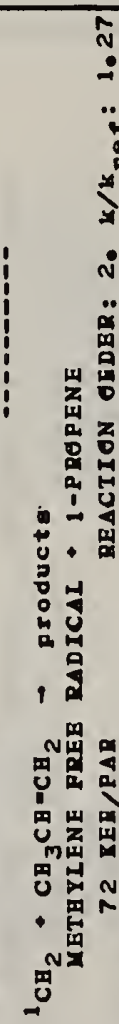 & 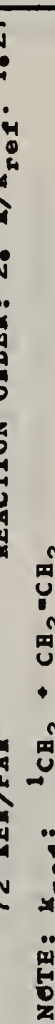 & 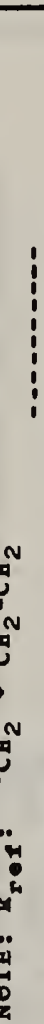 & 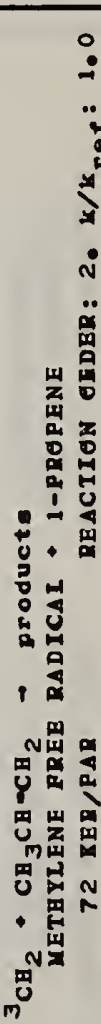 & 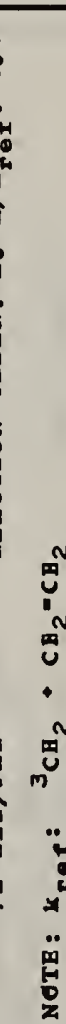 & | & 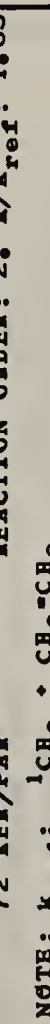 & 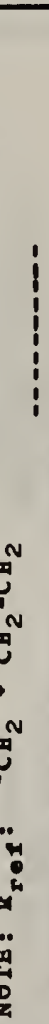 & 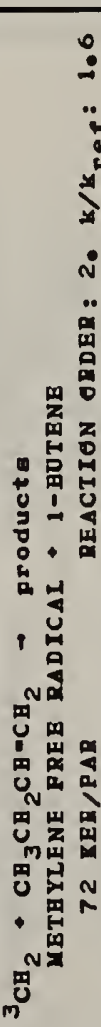 & 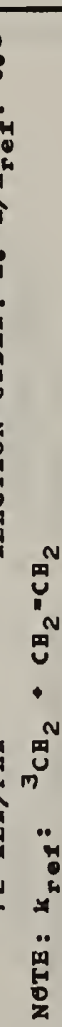 & $\begin{array}{l} \\
\end{array}$ & 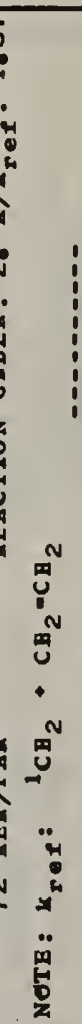 & $\begin{array}{l} \\
\end{array}$ & 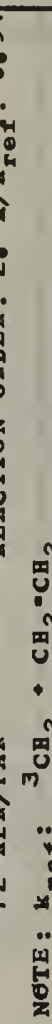 & 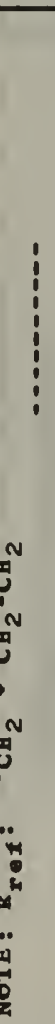 \\
\hline
\end{tabular}




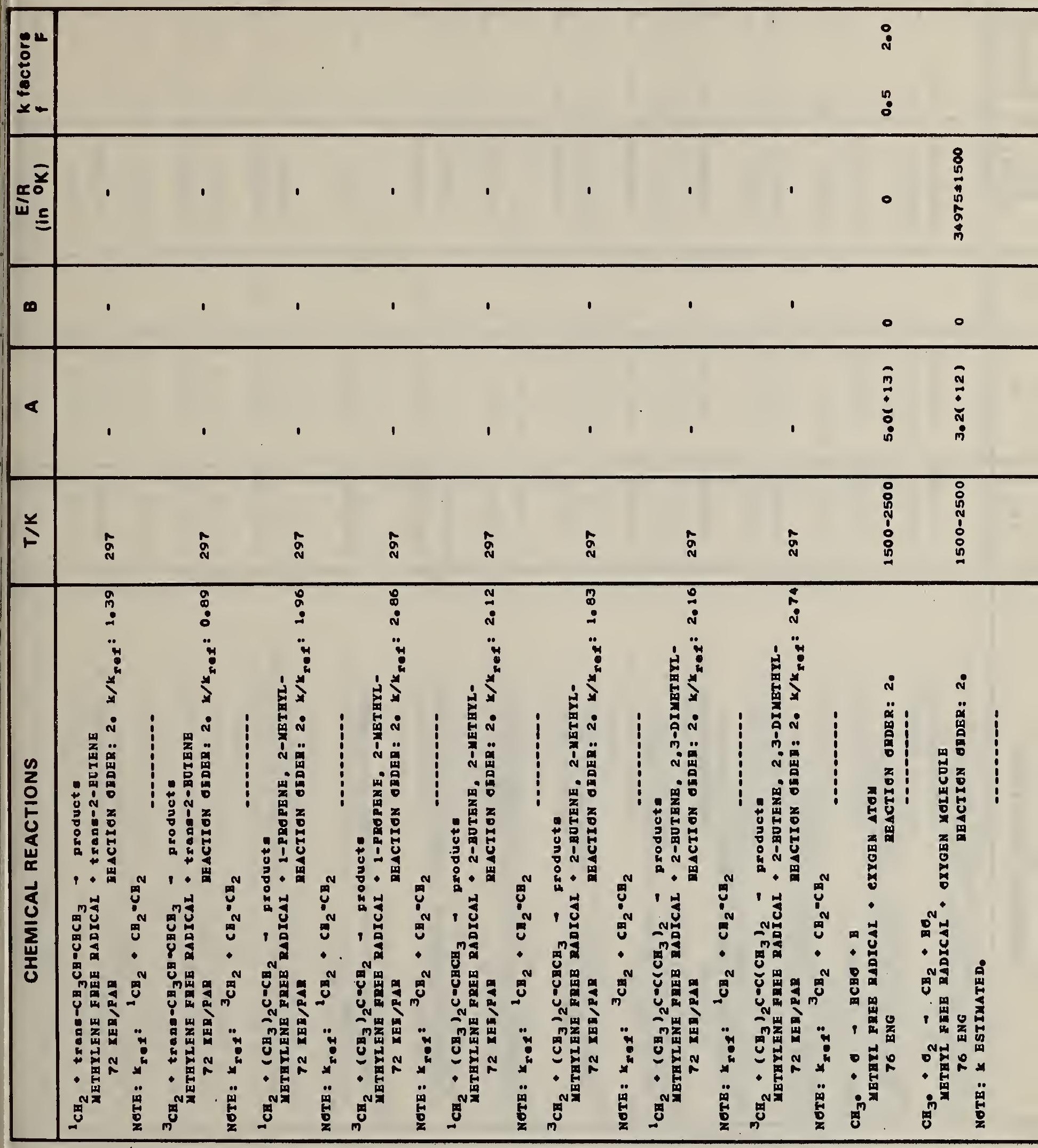




\begin{tabular}{|c|c|c|c|c|c|c|c|c|c|c|c|c|}
\hline 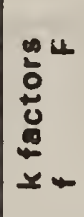 & $\stackrel{m}{:}$ & & : & $\stackrel{\text { : }}{:}$ & $\stackrel{\text { : }}{\circ}$ & $\begin{array}{l}\stackrel{m}{-} \\
\stackrel{0}{0}\end{array}$ & $\begin{array}{l}\text { : } \\
:\end{array}$ & $\stackrel{m}{0}$ & & $\begin{array}{l}\stackrel{n}{m} \\
\ddot{m} \\
\stackrel{m}{0}\end{array}$ & $\begin{array}{l}\stackrel{0}{\text { i }} \\
\stackrel{0}{0}\end{array}$ & $\begin{array}{l}\text { ก } \\
\stackrel{0}{\circ}\end{array}$ \\
\hline 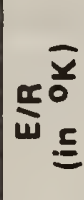 & $\begin{array}{l}: \\
: \\
0 \\
: \\
: \\
: \\
\vdots\end{array}$ & 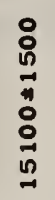 & 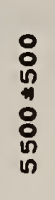 & 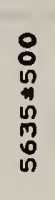 & $\begin{array}{l}: \\
\vdots \\
0 \\
0 \\
0 \\
0 \\
0\end{array}$ & 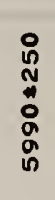 & 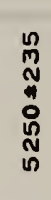 & $\begin{array}{l}\text { : } \\
\stackrel{h}{N} \\
\vdots \\
:\end{array}$ & ○ & 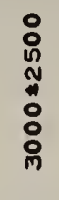 & 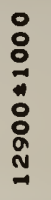 & 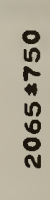 \\
\hline$\infty$ & 0 & 0 & 0 & 0 & 0 & 0 & ○ & $\stackrel{+}{:}$ & ○ & ! & - & ○ \\
\hline$<$ & 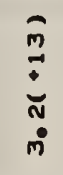 & 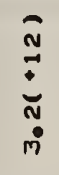 & 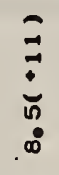 & $\bar{\vdots}$ & $\bar{\Xi}$ & 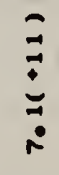 & $\begin{array}{l}\vdots \\
\vdots \\
\vdots\end{array}$ & $\begin{array}{l}0 \\
\vdots \\
\vdots \\
0\end{array}$ & $\begin{array}{c}\tilde{N} \\
\stackrel{n}{0} \\
\dot{n} \\
\dot{0}\end{array}$ & $\begin{array}{l}\Xi \\
\vdots \\
\vdots\end{array}$ & 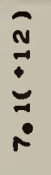 & $\begin{array}{l}\vdots \\
\vdots \\
\vdots\end{array}$ \\
\hline$\stackrel{Y}{F}$ & 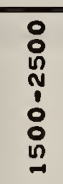 & $\begin{array}{l}0 \\
\stackrel{0}{0} \\
\stackrel{N}{1} \\
\vdots \\
0 \\
0\end{array}$ & $\begin{array}{l}\stackrel{\vdots}{0} \\
\vdots \\
\vdots \\
\vdots \\
n\end{array}$ & $\begin{array}{l}\stackrel{\vdots}{1} \\
\vdots \\
\vdots \\
\vdots\end{array}$ & $\begin{array}{l}\stackrel{\vdots}{1} \\
\vdots \\
\vdots\end{array}$ & $\begin{array}{l}: \\
\vdots \\
\vdots \\
\vdots \\
\text { in }\end{array}$ & $\begin{array}{l}0 \\
\hat{1} \\
\vdots \\
\vdots \\
\vdots\end{array}$ & 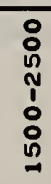 & $\begin{array}{l}: \\
: \\
\stackrel{\circ}{1} \\
\vdots \\
\vdots \\
0\end{array}$ & 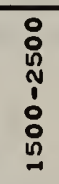 & 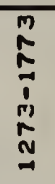 & $\begin{array}{l}: \\
\vdots \\
\vdots \\
\vdots\end{array}$ \\
\hline
\end{tabular}

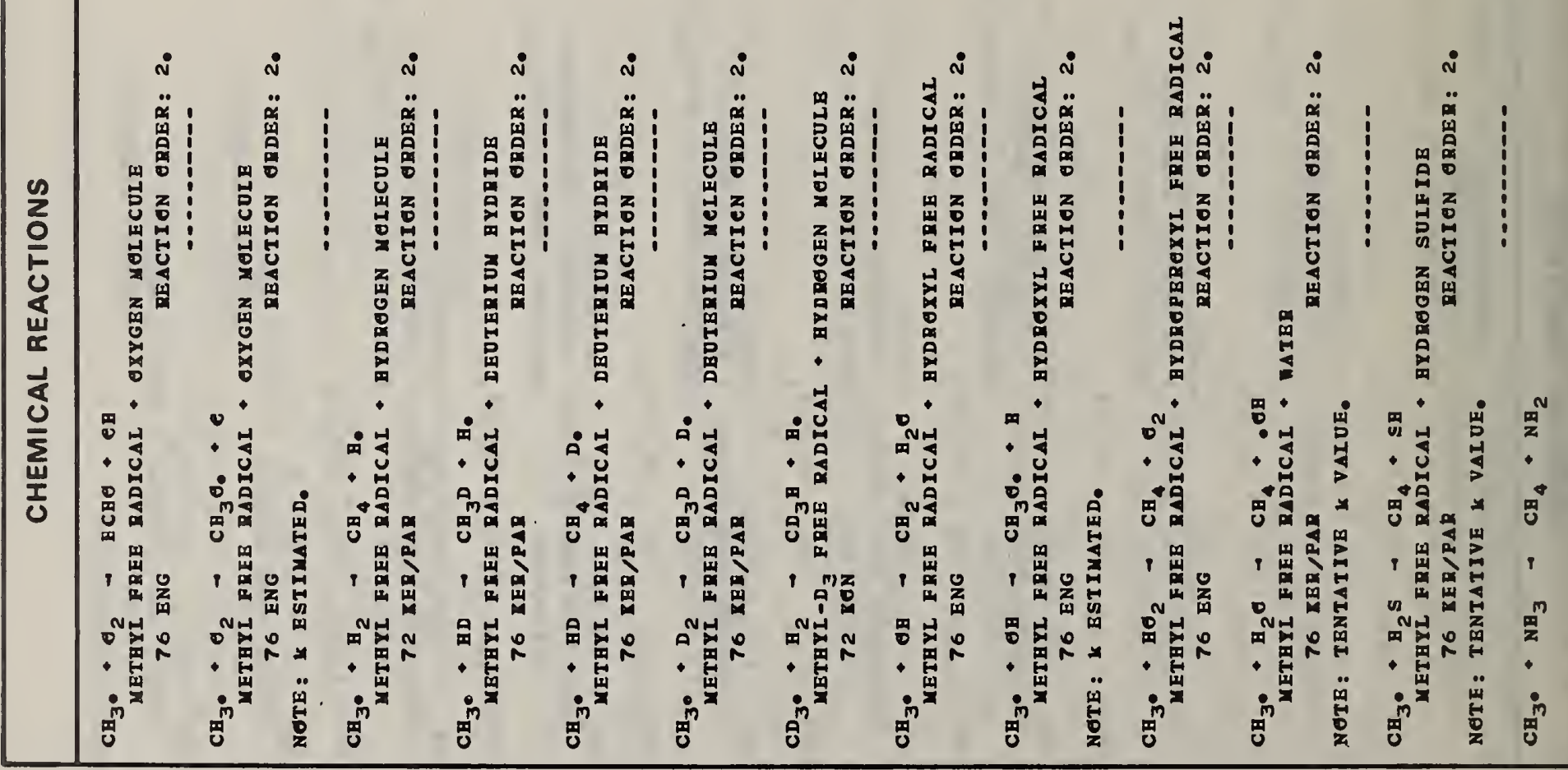




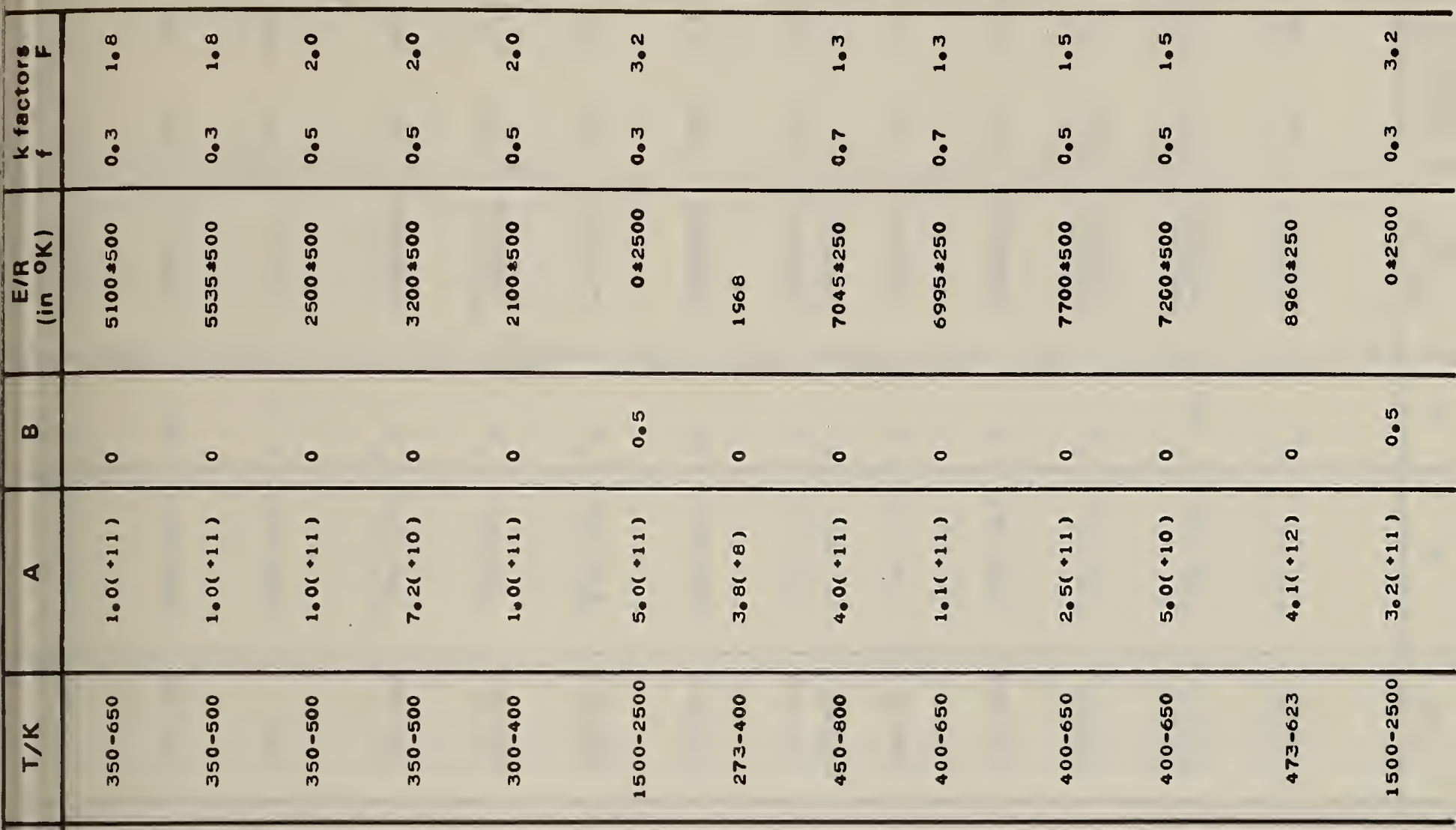

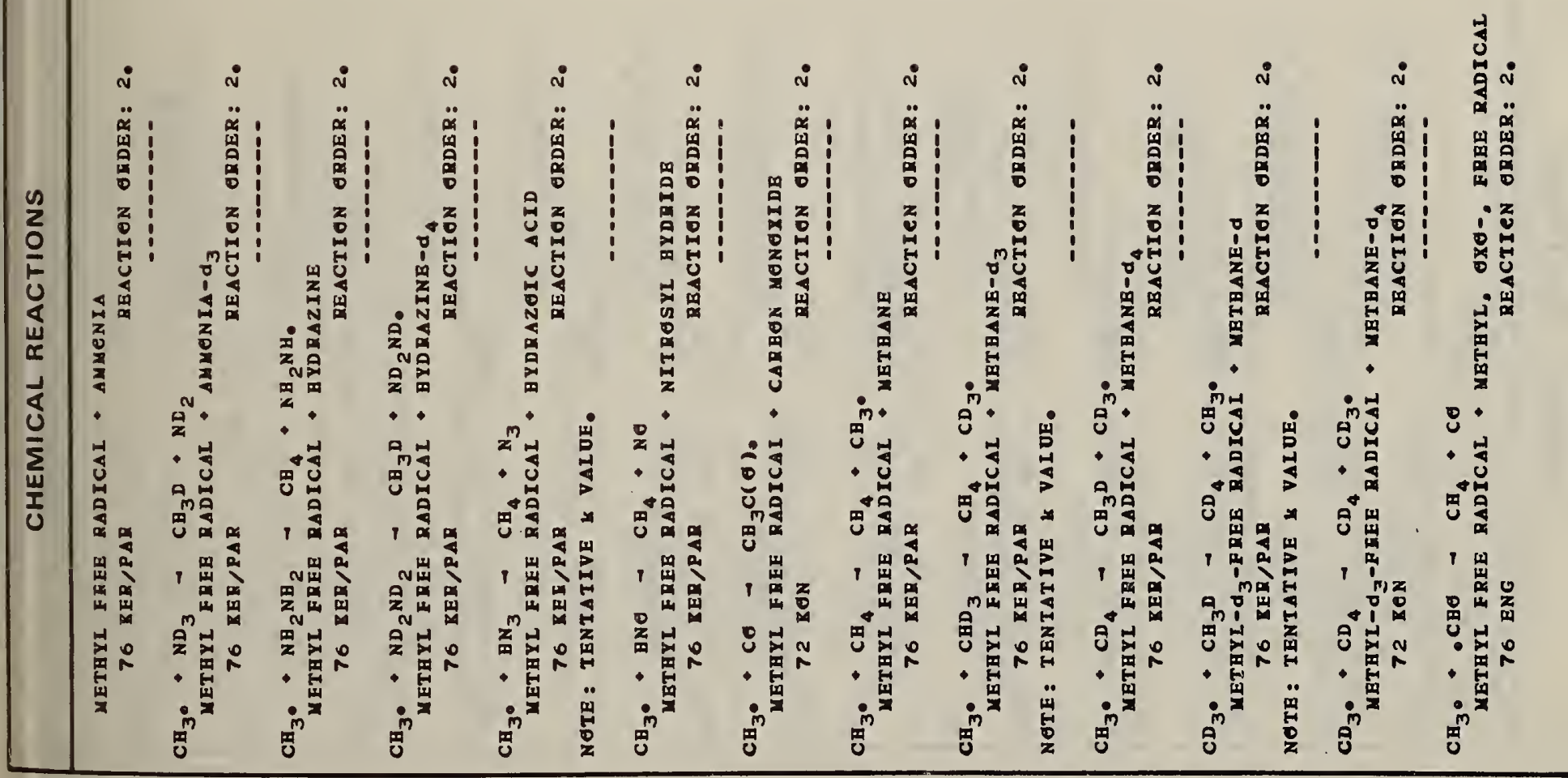




\begin{tabular}{|c|c|c|c|c|c|c|c|c|c|c|c|c|c|}
\hline $\begin{array}{l}0 \\
0 \\
0 \\
0 \\
0 \\
x\end{array}$ & $\begin{array}{l}? \\
\because \\
?\end{array}$ & $\begin{array}{l}n \\
m \\
m \\
m\end{array}$ & $\begin{array}{l}\text { n? } \\
\text { n }\end{array}$ & $\begin{array}{l}: \\
:\end{array}$ & $\begin{array}{l}: \\
:\end{array}$ & $:$ & $:$ & $\begin{array}{l}: \\
:\end{array}$ & $\begin{array}{l}: \\
\text { ก } \\
\text { : }\end{array}$ & $\begin{array}{l}: \\
\text { i } \\
\text { : }\end{array}$ & $\begin{array}{l}: \\
\text { ก } \\
\text { ! }\end{array}$ & $\begin{array}{l}: \\
\text { N } \\
\text { ! }\end{array}$ & $\begin{array}{l}0 \\
\text { i } \\
\text { ! }\end{array}$ \\
\hline $\begin{array}{r}\bar{x} \\
\text { wo } \\
=\end{array}$ & $\begin{array}{l}8 \\
0 \\
0 \\
0 \\
0 \\
0 \\
0 \\
m\end{array}$ & $\begin{array}{l}: \\
\stackrel{\circ}{0} \\
\stackrel{N}{0} \\
: \\
: \\
:\end{array}$ & $\begin{array}{l}\circ \\
\circ \\
n \\
\text { în } \\
n \\
n \\
n \\
m\end{array}$ & $\begin{array}{l}8 \\
: \\
: \\
8 \\
8 \\
0\end{array}$ & 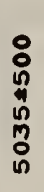 & 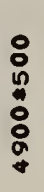 & $\begin{array}{l}0 \\
0 \\
n \\
0 \\
0 \\
0 \\
0 \\
0\end{array}$ & $\begin{array}{l}: \\
: \\
0 \\
: \\
: \\
\vdots \\
0\end{array}$ & $\begin{array}{l}: \\
: \\
01 \\
: \\
: \\
:\end{array}$ & $\begin{array}{l}: \\
: \\
\vdots \\
\vdots \\
\vdots \\
0 \\
\text { is }\end{array}$ & I & $\begin{array}{l}: \\
\text { in } \\
\text { : } \\
\text { in } \\
\text { o } \\
\text { N }\end{array}$ & 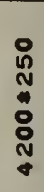 \\
\hline$\infty$ & 0 & $\because$ & 0 & 0 & 0 & 0 & 0 & 0 & 0 & 0 & I & 0 & 0 \\
\hline$<$ & $\begin{array}{l} \pm \\
\Xi\end{array}$ & $\begin{array}{l}0 \\
\vdots \\
0\end{array}$ & $\begin{array}{l}\square \\
\vdots\end{array}$ & $\begin{array}{l}\hat{0} \\
\dot{N} \\
\dot{0}\end{array}$ & $\begin{array}{l}= \\
\bar{a} \\
\vdots\end{array}$ & $\begin{array}{l}\vec{\vdots} \\
\bar{m} \\
\dot{m}\end{array}$ & $\begin{array}{l}\vdots \\
\vdots \\
\dot{0}\end{array}$ & $\frac{0}{\vdots}$ & $\begin{array}{l}\square \\
\vdots \\
\vdots\end{array}$ & 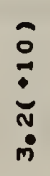 & 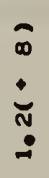 & $=$ & $\begin{array}{l}0 \\
\vdots \\
0 \\
0\end{array}$ \\
\hline & $\begin{array}{l}\text { : } \\
\text { ? } \\
\text { ! } \\
\text { : } \\
m\end{array}$ & 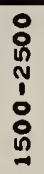 & $\begin{array}{l}: \\
: \\
0 \\
\vdots \\
: \\
\text { in }\end{array}$ & 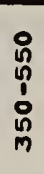 & $\begin{array}{l}\circ \\
\text { ஜn } \\
\vdots \\
\vdots \\
\text { in }\end{array}$ & $\begin{array}{l}\text { 유 } \\
\text { ! } \\
\text { 음 } \\
\text { m }\end{array}$ & $\begin{array}{l}0 \\
0 \\
0 \\
! \\
0 \\
\text { p }\end{array}$ & 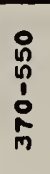 & $\begin{array}{l}\text { : } \\
\text { ! } \\
\text { : } \\
0\end{array}$ & $\begin{array}{l}: \\
0 \\
! \\
\vdots \\
\vdots\end{array}$ & $\begin{array}{l}m \\
\text { mo } \\
\text { m }\end{array}$ & $\begin{array}{l}: \\
\text { ! } \\
\vdots \\
:\end{array}$ & $\begin{array}{l}8 \\
\text { in } \\
1 \\
\vdots \\
0\end{array}$ \\
\hline
\end{tabular}

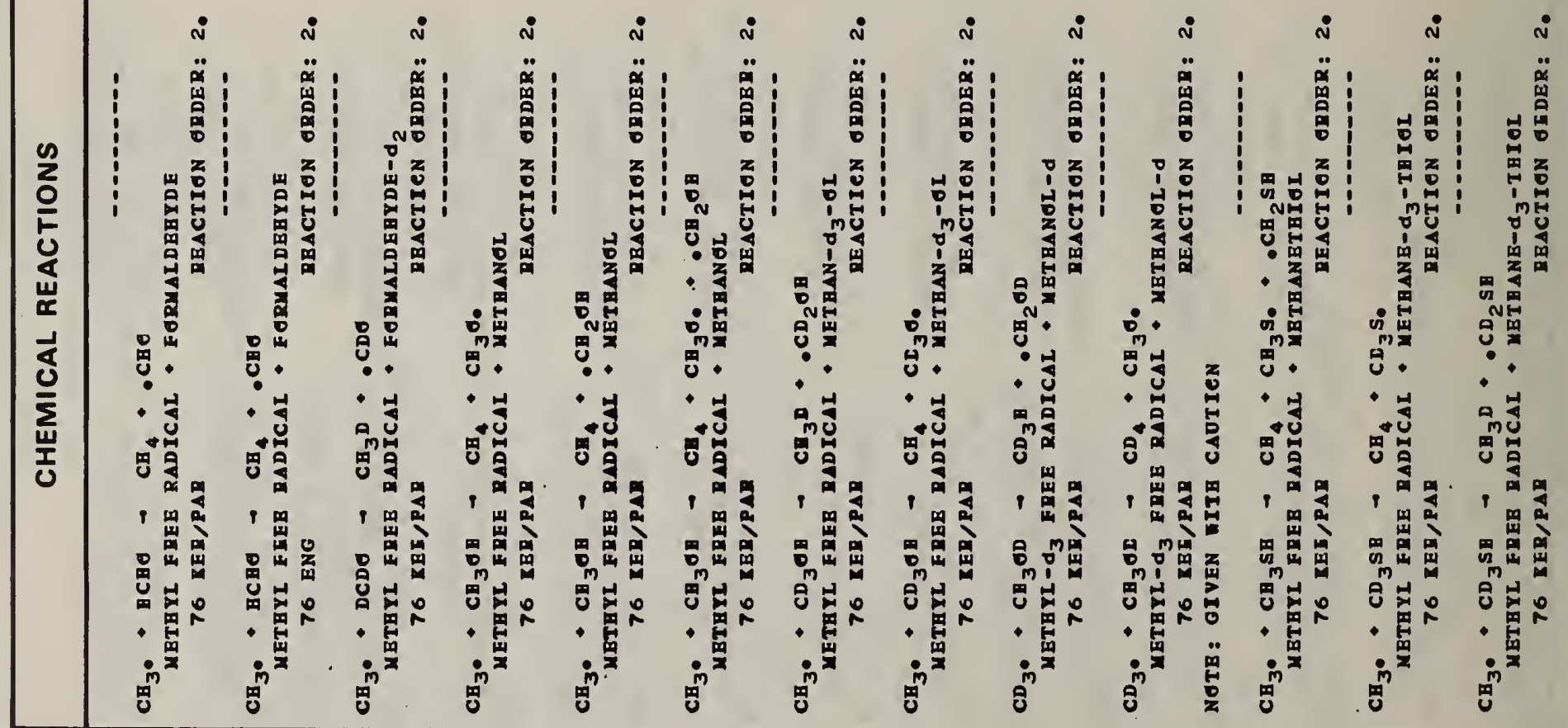




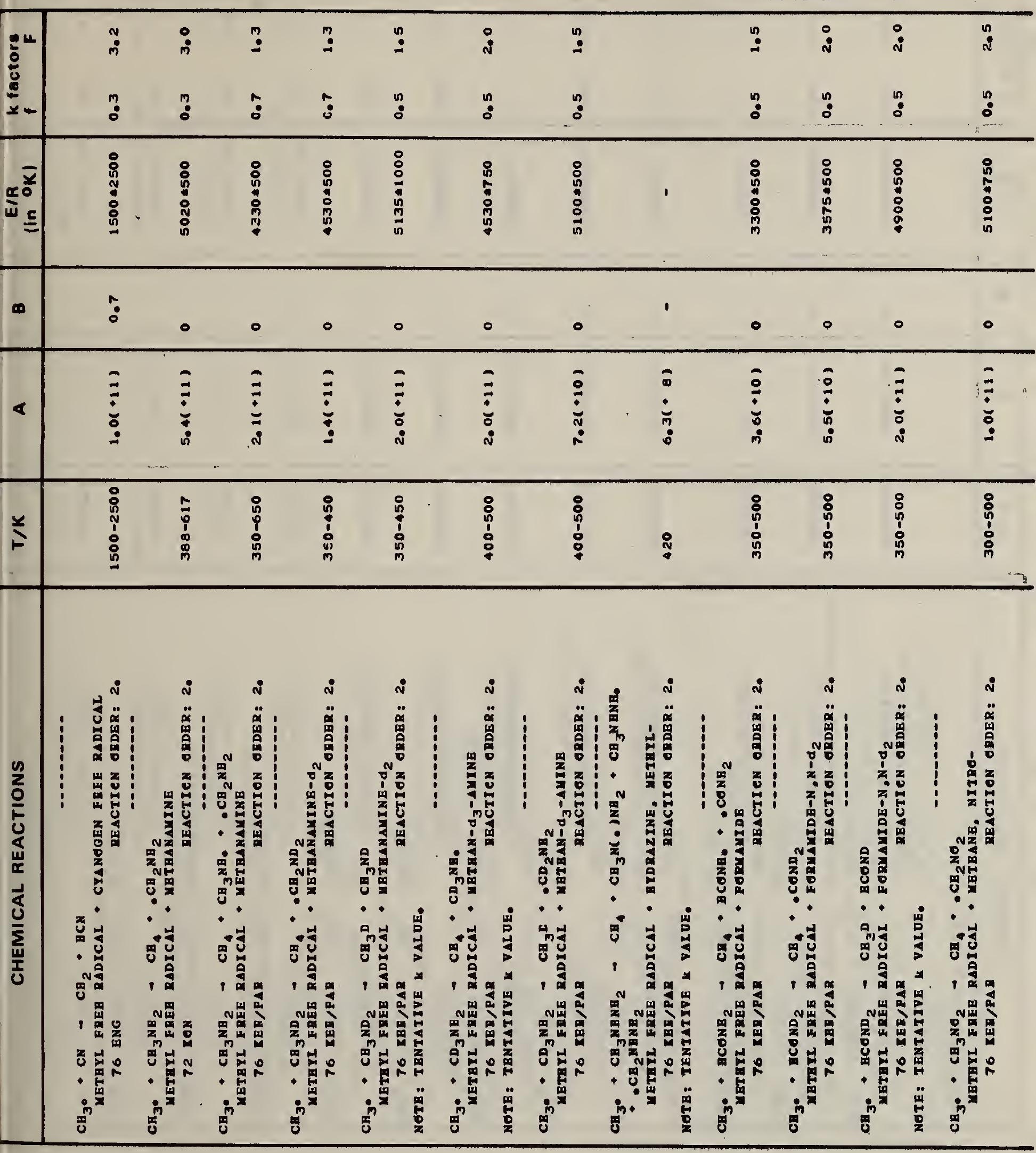




\begin{tabular}{|c|c|c|c|c|c|c|c|c|c|c|c|c|c|c|c|}
\hline 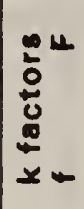 & $\begin{array}{l}\stackrel{n}{:} \\
\stackrel{0}{0}\end{array}$ & $\begin{array}{l}\stackrel{0}{:} \\
\stackrel{0}{0}\end{array}$ & & & & $\begin{array}{l}\stackrel{?}{:} \\
\stackrel{n}{0}\end{array}$ & & & $\begin{array}{l}\stackrel{m}{:} \\
\stackrel{0}{\circ} \\
\stackrel{0}{0}\end{array}$ & $\begin{array}{l}\text { : } \\
:\end{array}$ & $\begin{array}{l}\text { : } \\
\stackrel{0}{0}\end{array}$ & $\begin{array}{l}: \\
:\end{array}$ & $\begin{array}{l}: \\
:\end{array}$ & $\begin{array}{l}\text { m: } \\
\text { : }\end{array}$ & \\
\hline 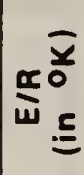 & 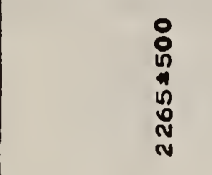 & 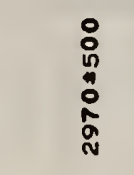 & $\stackrel{8}{\pi}$ & & 吕 & $\begin{array}{l}: \\
: \\
1 \\
: \\
: \\
:\end{array}$ & 吕 & & 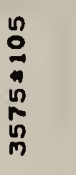 & 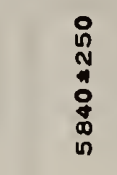 & $\begin{array}{l}\stackrel{0}{N} \\
\stackrel{N}{0} \\
: \\
: \\
:\end{array}$ & $\begin{array}{l}0 \\
\stackrel{0}{0} \\
\stackrel{1}{0} \\
0 \\
0 \\
0 \\
0\end{array}$ & $\begin{array}{l}\stackrel{\circ}{n} \\
\vdots \\
\vdots \\
\vdots \\
\vdots\end{array}$ & $\begin{array}{l}0 \\
\stackrel{5}{N} \\
\vdots \\
\vdots \\
\dot{0} \\
\infty \\
0\end{array}$ & \\
\hline$\infty$ & $\circ$ & ० & 1 & & ० & ० & ○ & & 0 & $\circ$ & - & $\circ$ & $\circ$ & $\circ$ & \\
\hline$<$ & $\begin{array}{l}0 \\
\vdots \\
\vdots \\
\vdots \\
\vdots\end{array}$ & $\begin{array}{l}0 \\
\vdots \\
\vdots \\
\dot{m} \\
\dot{m}\end{array}$ & ' & & $\begin{array}{l}\vdots \\
\vdots \\
\vdots \\
\vdots \\
\vdots\end{array}$ & $\begin{array}{l}\vdots \\
\vdots \\
\vdots \\
\vdots\end{array}$ & $\vec{m}$ & & $\begin{array}{l}\bar{\vdots} \\
\vdots \\
\vdots \\
0\end{array}$ & $\underset{\vdots}{\vdots}$ & $\begin{array}{l}\overline{7} \\
\vdots \\
0\end{array}$ & $\stackrel{\grave{N}}{\vdots}$ & $\begin{array}{l}\bar{\vdots} \\
\vdots \\
\dot{0}\end{array}$ & $\begin{array}{l}\bar{Z} \\
\vdots \\
\vdots \\
\vdots \\
\vdots\end{array}$ & \\
\hline$\stackrel{ }{F}$ & $\begin{array}{l}\text { : } \\
0 \\
\vdots \\
\vdots \\
0\end{array}$ & $\begin{array}{l}: \\
\dot{1} \\
1 \\
\vdots \\
\dot{n}\end{array}$ & 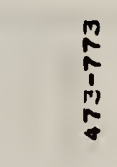 & & 交 & 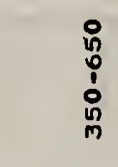 & 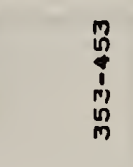 & & 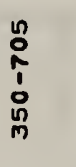 & $\begin{array}{l}: \\
\vdots \\
\vdots \\
\vdots \\
\vdots \\
\vdots\end{array}$ & $\begin{array}{l}: \\
\vdots \\
\vdots \\
\vdots \\
: \\
i\end{array}$ & $\begin{array}{l}0 \\
\vdots \\
0 \\
1 \\
0 \\
0 \\
n \\
n\end{array}$ & 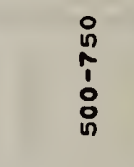 & 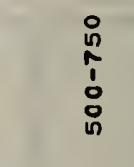 & \\
\hline 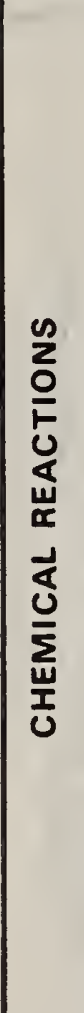 & 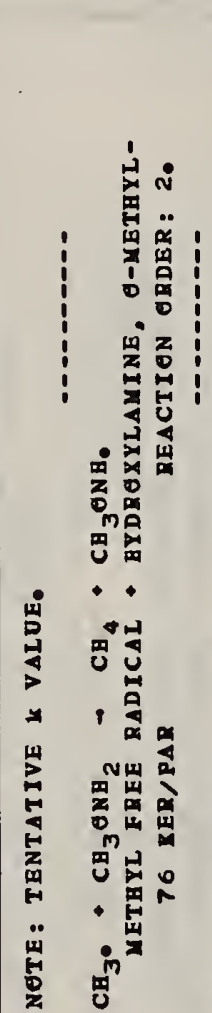 & 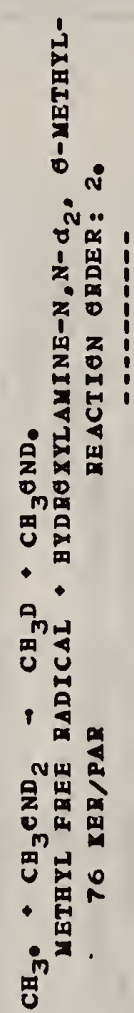 & 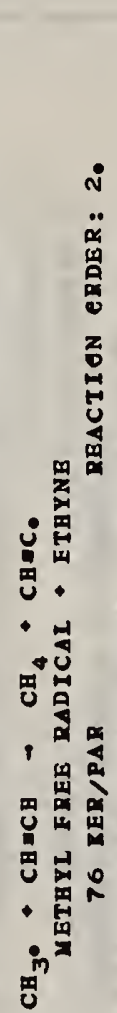 & 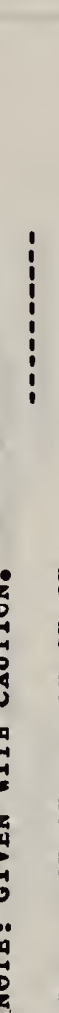 & 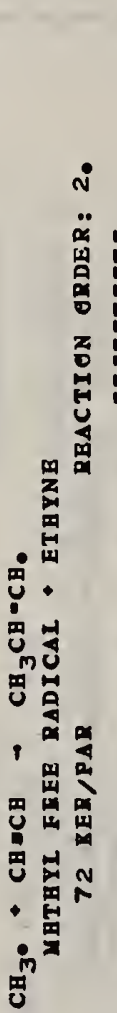 & 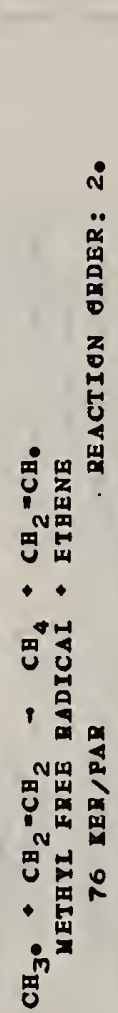 & 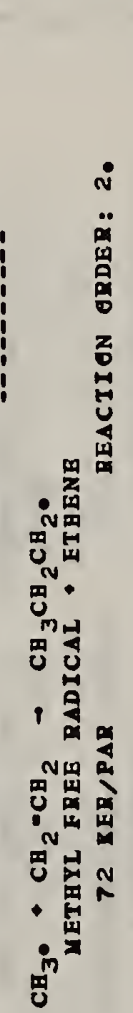 & 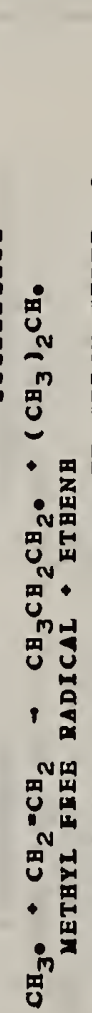 & 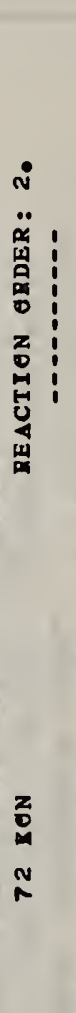 & 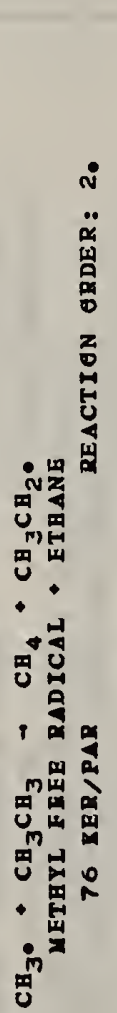 & 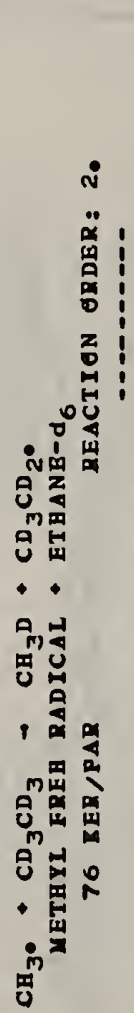 & $\begin{array}{r}r \\
\\
\end{array}$ & 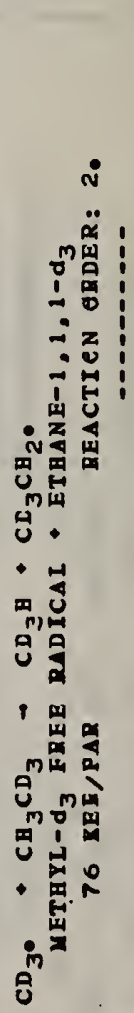 & 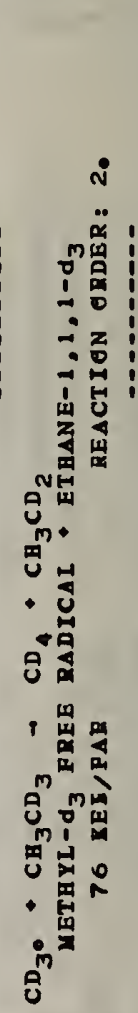 & $\begin{array}{l}\vdots \\
\vdots \\
0 \\
0 \\
0\end{array}$ \\
\hline
\end{tabular}




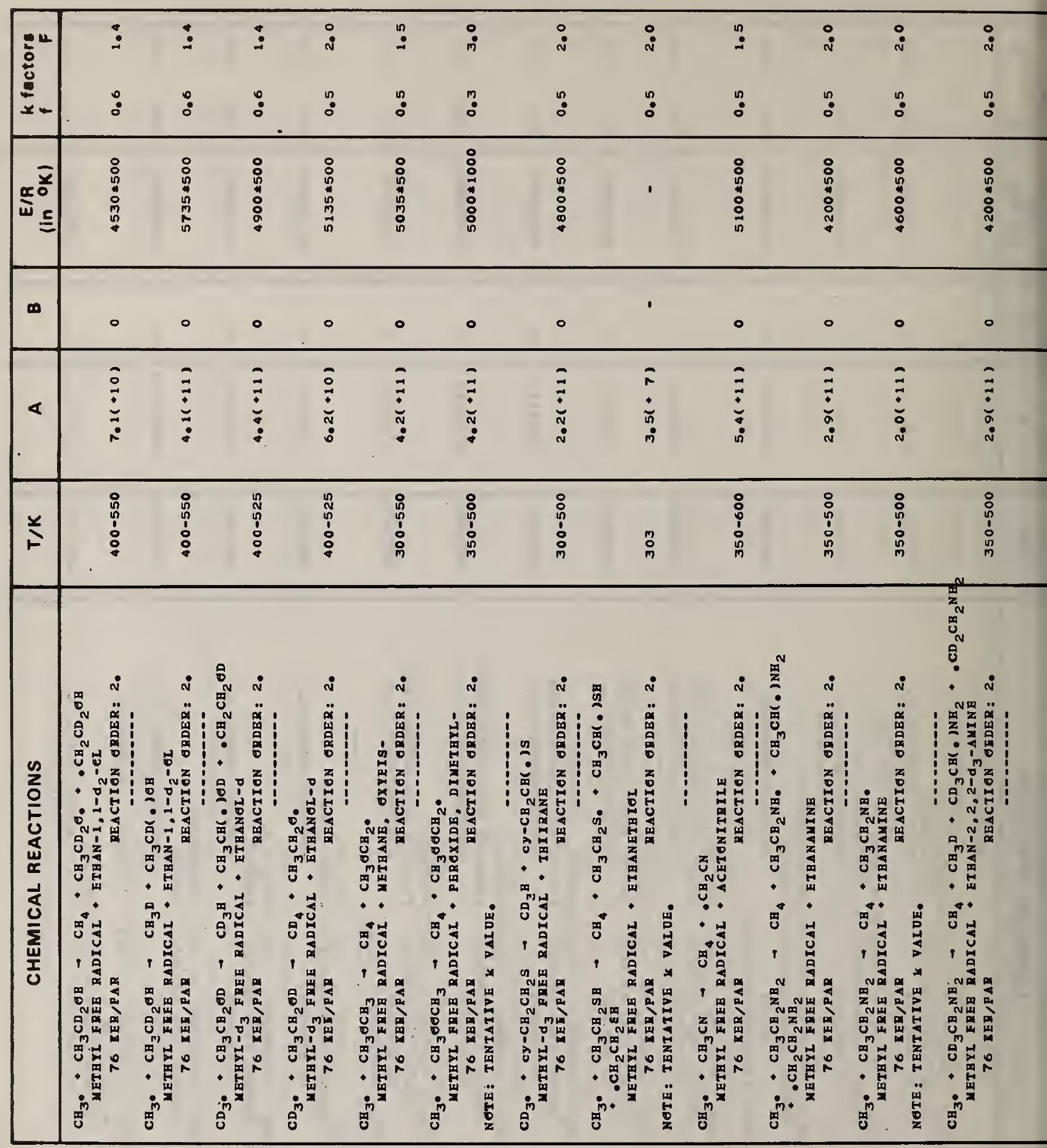




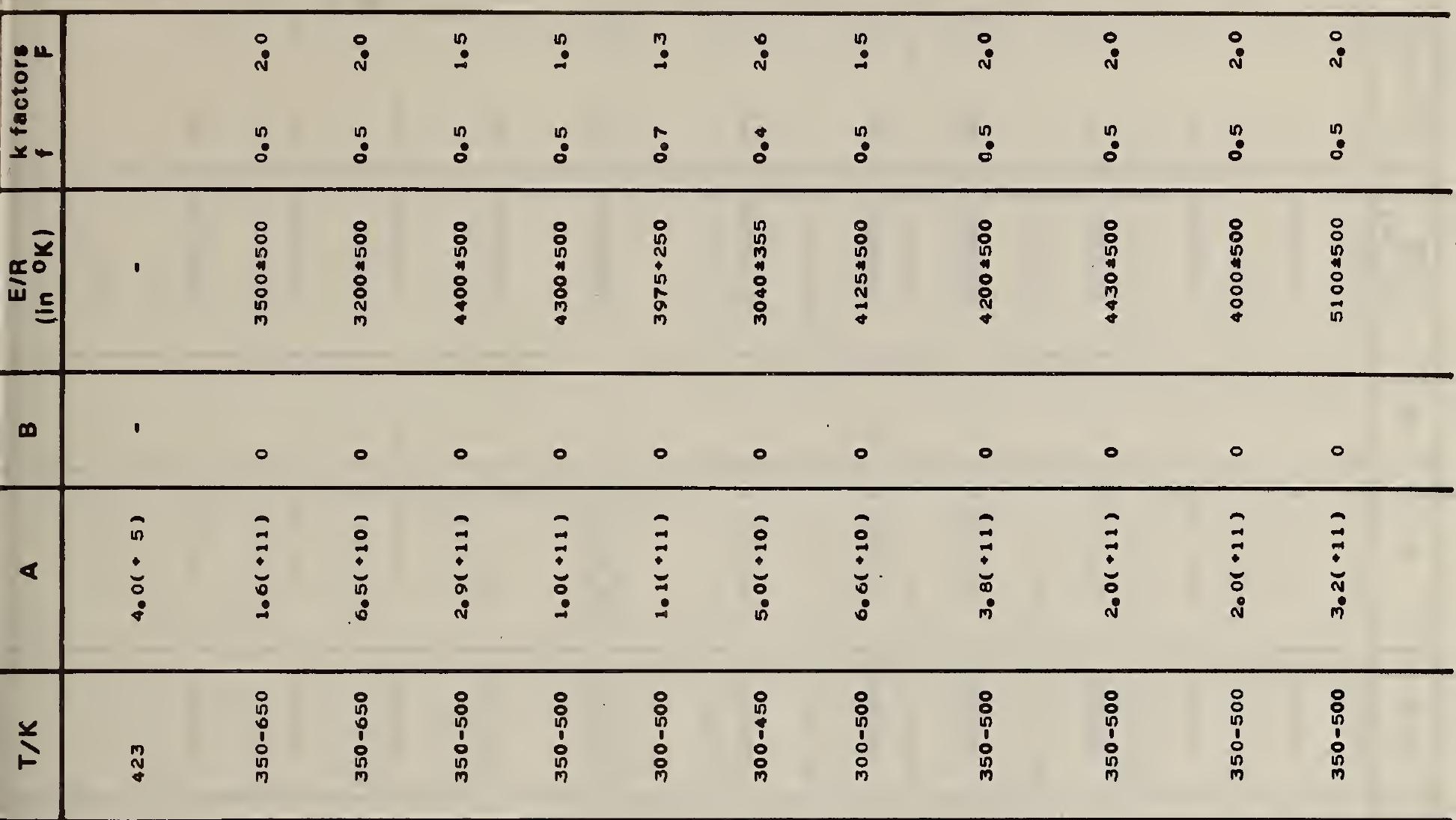

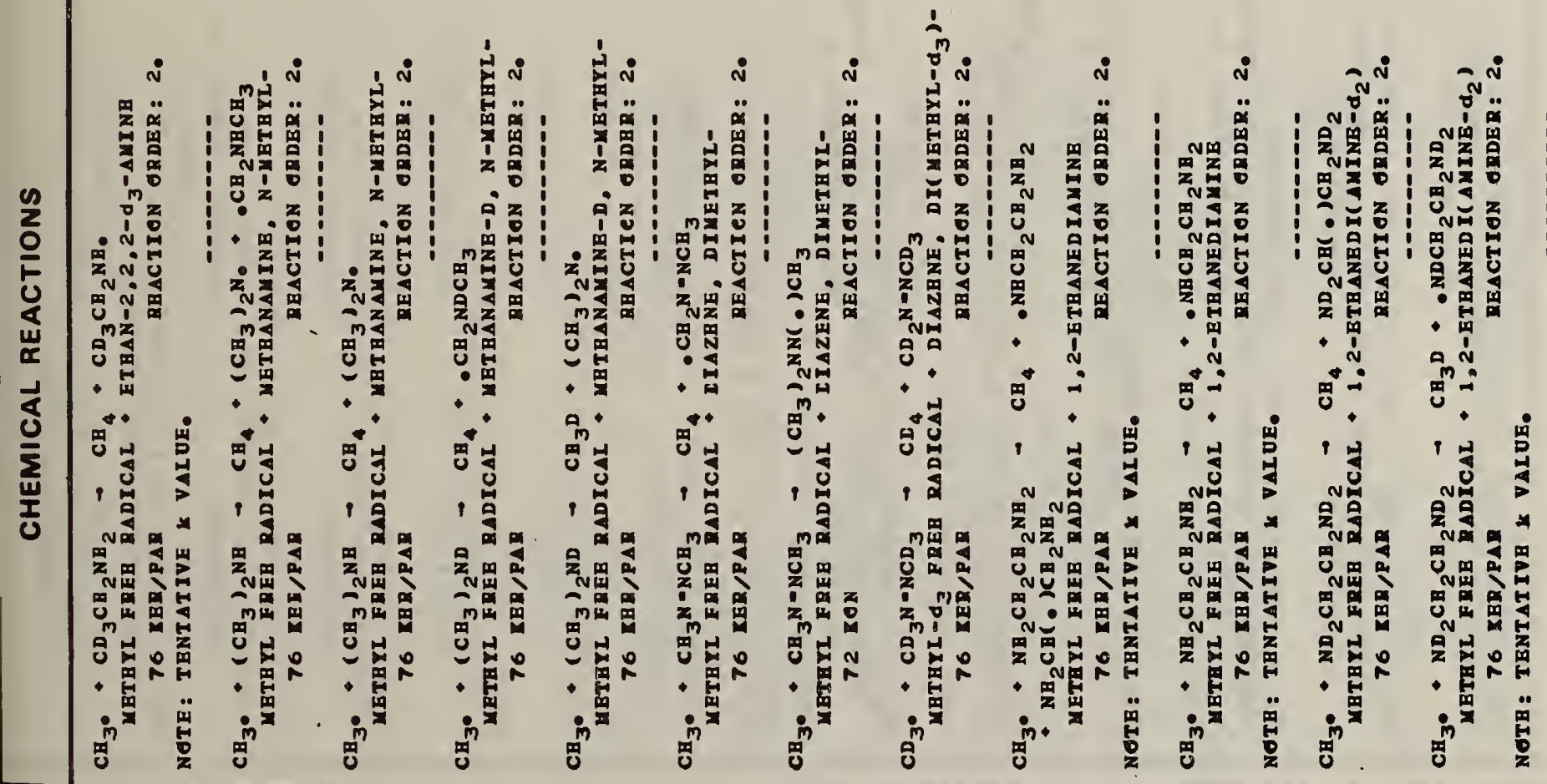




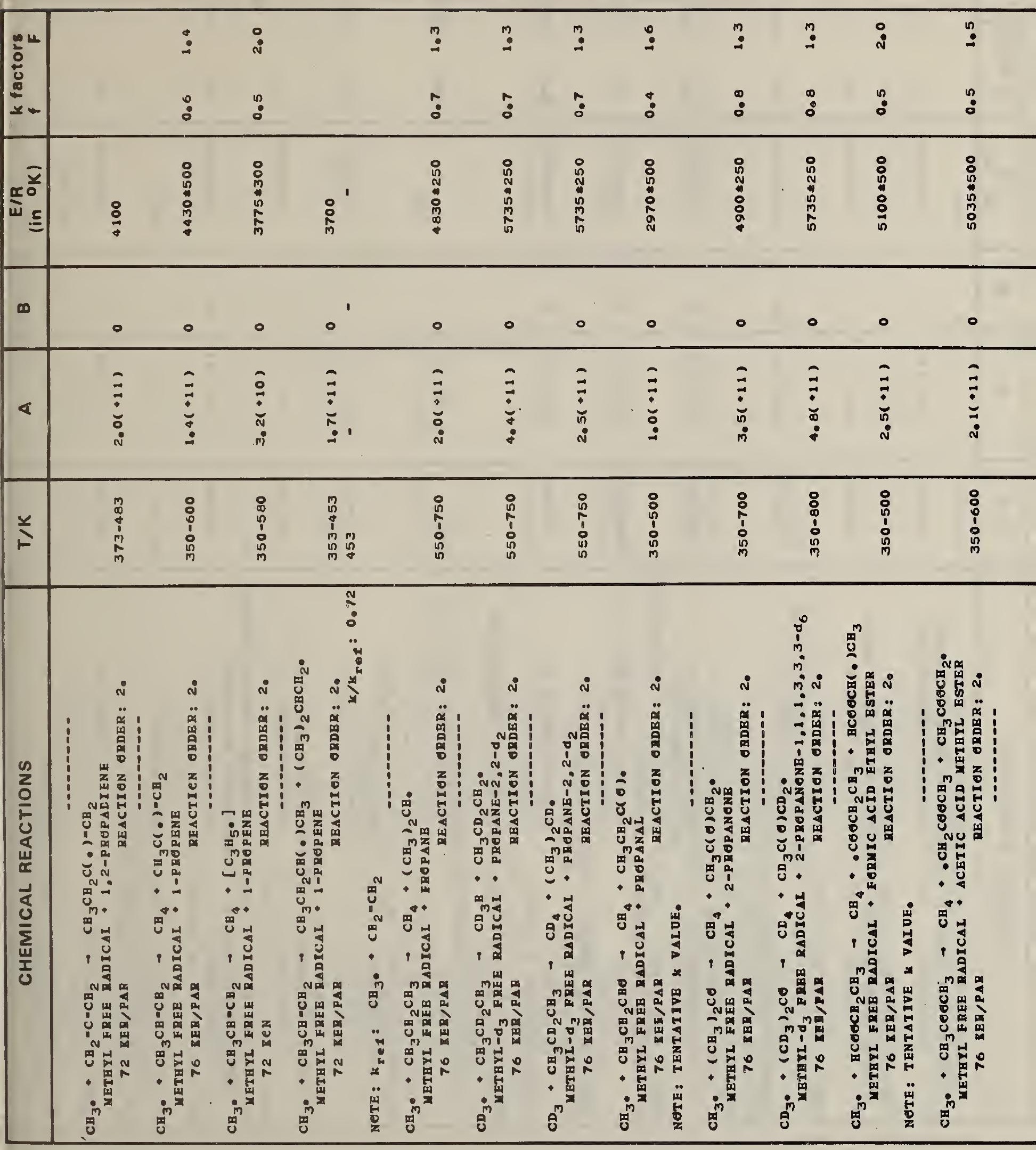




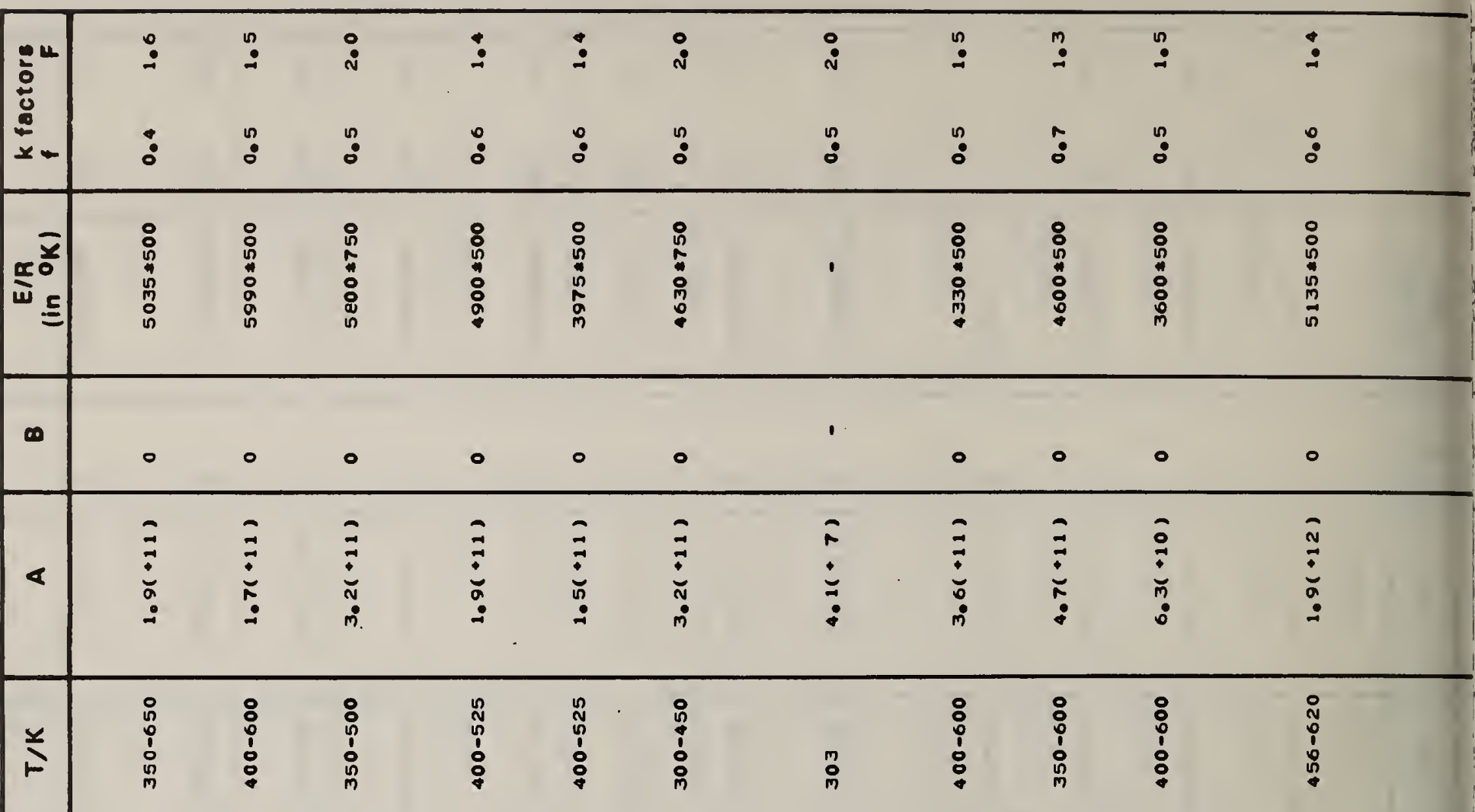

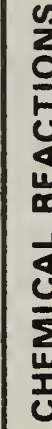

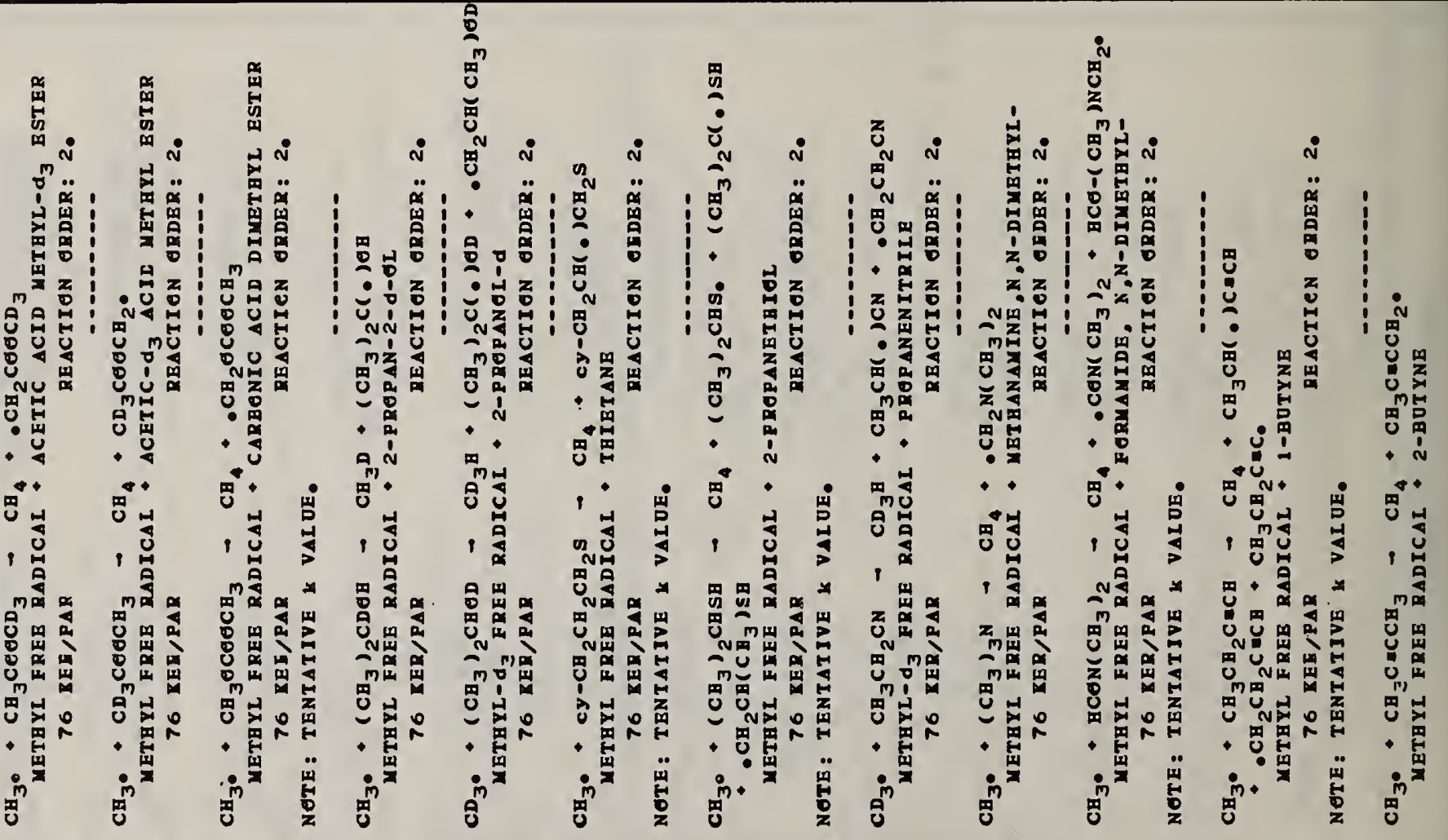




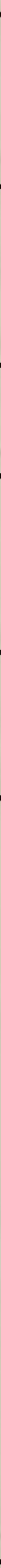




\begin{tabular}{|c|c|c|c|c|c|c|c|c|c|c|c|c|}
\hline 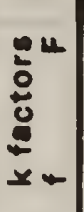 & $\begin{array}{l}\stackrel{m}{-} \\
\stackrel{0}{0}\end{array}$ & $\begin{array}{l}\stackrel{\vdots}{:} \\
:\end{array}$ & $\stackrel{m}{\circ}$ & $\stackrel{m}{:}$ & $\begin{array}{l}\stackrel{\circ}{*} \\
\stackrel{0}{:}\end{array}$ & $\stackrel{m}{:}$ & $\begin{array}{l}\stackrel{m}{-} \\
\text { : }\end{array}$ & $\begin{array}{l}\stackrel{m}{:} \\
\stackrel{0}{0}\end{array}$ & $\stackrel{n}{\text { in }}$ & $\begin{array}{l}? \\
: \\
:\end{array}$ & $\begin{array}{l}\vdots \\
\vdots\end{array}$ & $\begin{array}{l}\stackrel{0}{0} \\
\vdots\end{array}$ \\
\hline 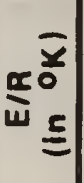 & 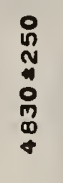 & $\begin{array}{l}0 \\
\vdots \\
\vdots \\
0 \\
\vdots \\
\vdots \\
\vdots\end{array}$ & 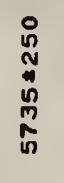 & 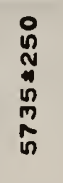 & $\begin{array}{l}: \\
: \\
10 \\
: \\
\vdots \\
\vdots\end{array}$ & $\begin{array}{l}\stackrel{\circ}{n} \\
\stackrel{n}{n} \\
\hat{n} \\
\vdots \\
n\end{array}$ & 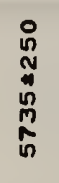 & 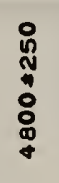 & 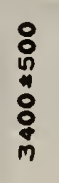 & 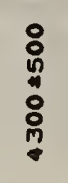 & 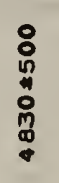 & $\begin{array}{l}\circ \\
\stackrel{0}{0} \\
\vdots \\
0 \\
\vdots \\
\text { N }\end{array}$ \\
\hline$\infty$ & 0 & 0 & & 0 & 0 & 0 & 0 & 0 & 0 & ० & 0 & - \\
\hline$\ll$ & $\begin{array}{l}\vdots \\
\vdots \\
\vdots\end{array}$ & 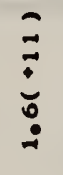 & $\begin{array}{l}\bar{\vdots} \\
\vdots \\
\vdots \\
\vdots\end{array}$ & $\underset{\vdots}{\vdots}$ & $\begin{array}{l}0 \\
\vdots \\
\dot{n} \\
\dot{\infty}\end{array}$ & $\begin{array}{l}0 \\
\vdots \\
0 \\
0\end{array}$ & $\begin{array}{l}\beth \\
\vdots \\
\vdots\end{array}$ & $\bar{\Xi}$ & $\bar{\Xi}$ & $\underset{\vdots}{\vdots}$ & $\bar{\Xi}$ & $\vdots$ \\
\hline$\stackrel{\text { }}{\vDash}$ & 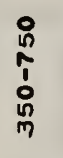 & 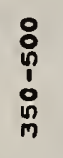 & $\begin{array}{l}0 \\
\stackrel{n}{n} \\
\grave{\vdots} \\
\vdots\end{array}$ & $\begin{array}{l}0 \\
\stackrel{n}{n} \\
\hat{\vdots} \\
\vdots \\
0\end{array}$ & $\begin{array}{l}\text { : } \\
\stackrel{\varphi}{0} \\
\vdots \\
\vdots \\
n\end{array}$ & 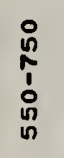 & 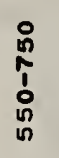 & 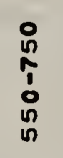 & 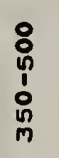 & $\begin{array}{l}\stackrel{0}{0} \\
\vdots \\
\vdots \\
\vdots \\
\vdots\end{array}$ & $\begin{array}{l}: \\
\vdots \\
\vdots \\
\vdots \\
\vdots\end{array}$ & $\begin{array}{l}: \\
: \\
\vdots \\
\vdots \\
0 \\
n\end{array}$ \\
\hline
\end{tabular}

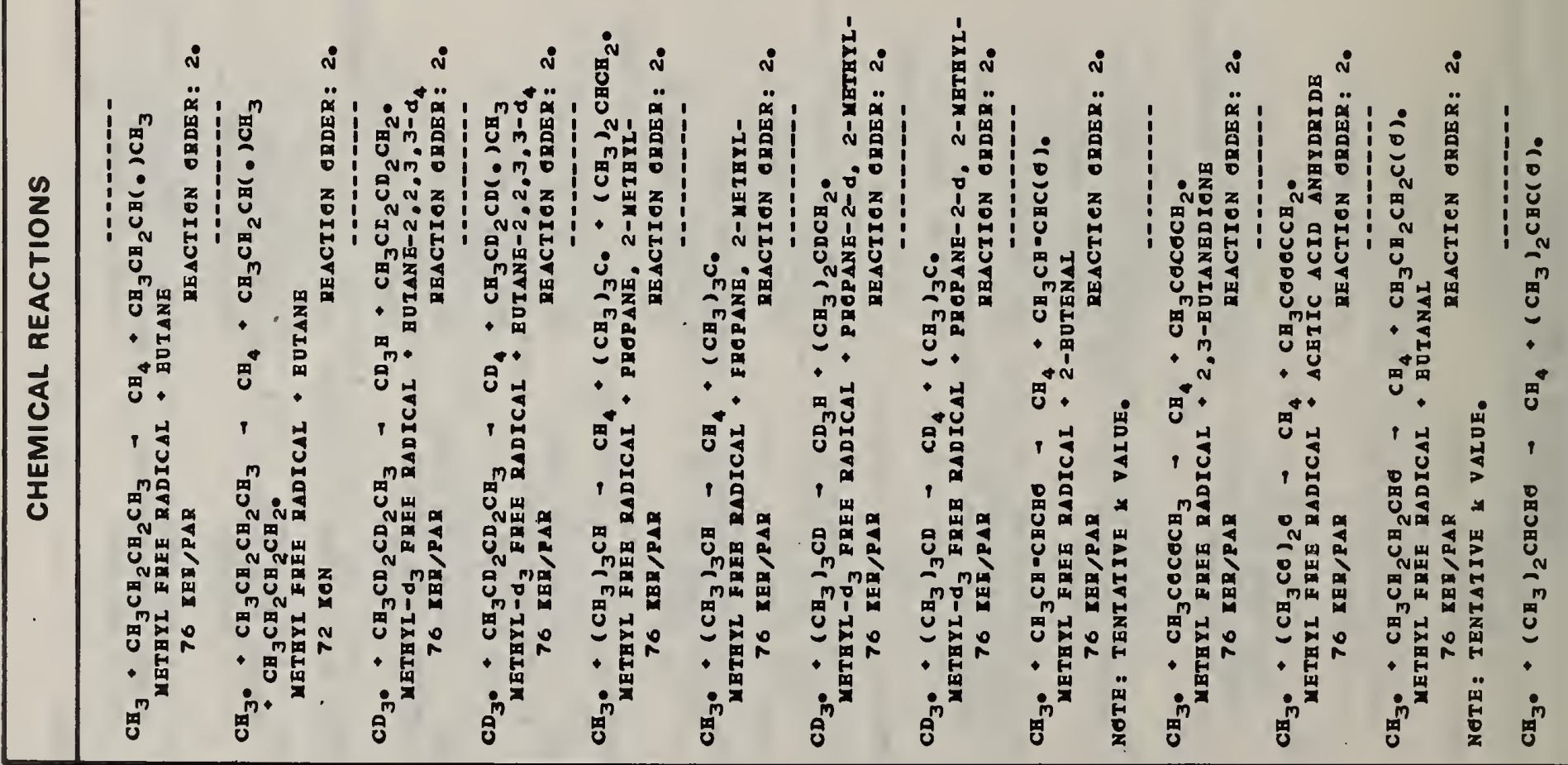




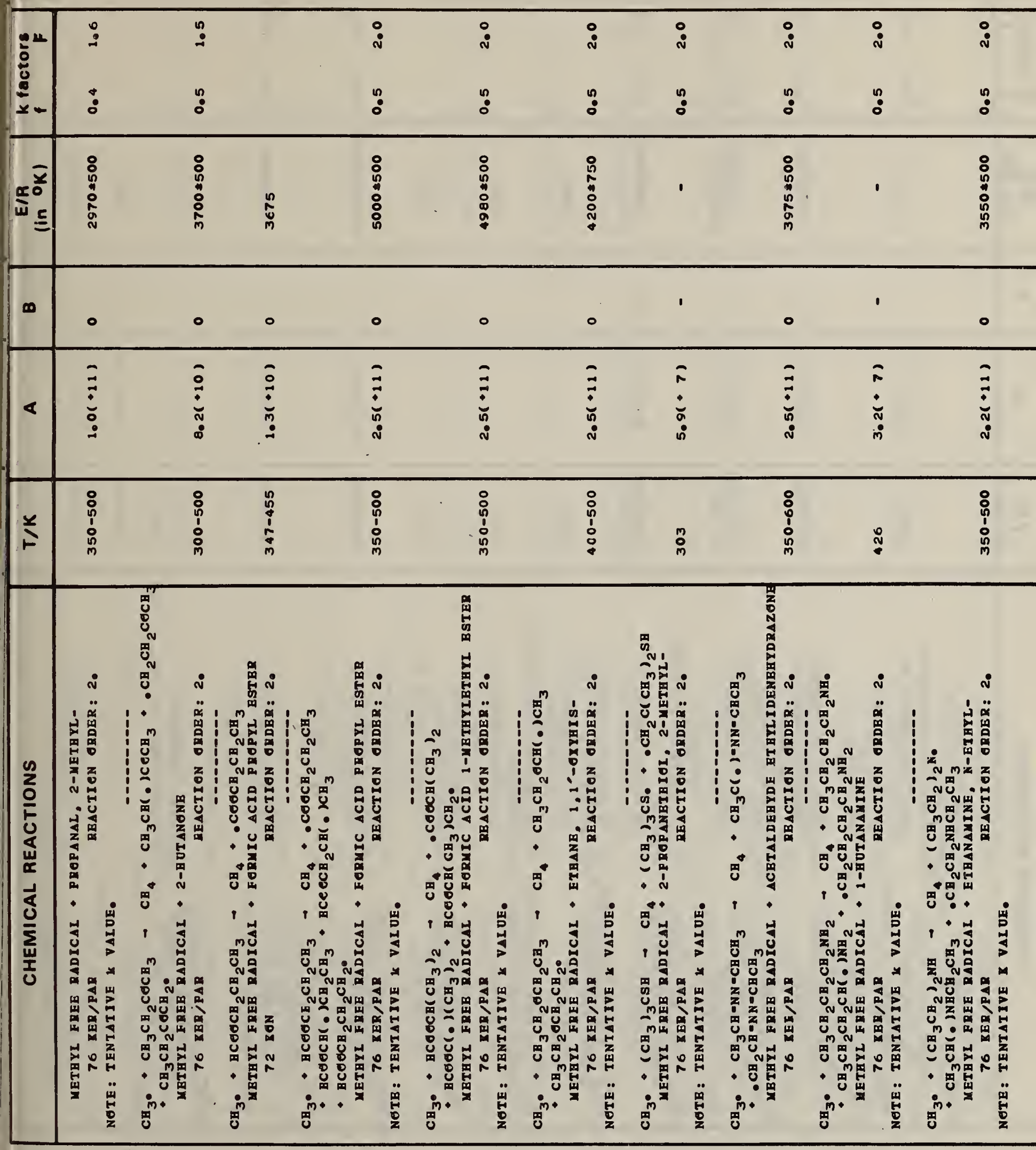




\begin{tabular}{|c|c|c|c|c|c|c|c|c|c|c|c|}
\hline $\begin{array}{l}04 \\
\frac{6}{0} \\
0 \\
0 \\
0 \\
x \\
x-\end{array}$ & $\begin{array}{l}? \\
\because \\
\because\end{array}$ & $\begin{array}{l}? \\
\because \\
:\end{array}$ & $\begin{array}{l}? \\
\therefore \\
\therefore\end{array}$ & $\begin{array}{l}? \\
\because \\
\because\end{array}$ & $\begin{array}{l}? \\
\because \\
\therefore\end{array}$ & $\begin{array}{l}: \\
:\end{array}$ & $\begin{array}{l}: \\
: \\
:\end{array}$ & $\begin{array}{l}: \\
\text { i } \\
\text { ? }\end{array}$ & $\begin{array}{l}: \\
:\end{array}$ & $\begin{array}{l}0 \\
\text { s } \\
? \\
:\end{array}$ & $\begin{array}{l}? \\
? \\
?\end{array}$ \\
\hline$\sigma^{\underline{\underline{y}}}$ & 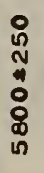 & 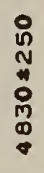 & 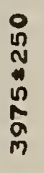 & 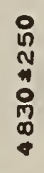 & 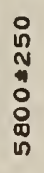 & 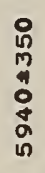 & 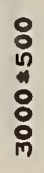 & $\begin{array}{l}: \\
: \\
0 \\
: \\
: \\
\stackrel{N}{0} \\
m\end{array}$ & $\begin{array}{l}8 \\
0 \\
01 \\
0 \\
0 \\
0 \\
0\end{array}$ & $\begin{array}{l}8 \\
0 \\
0 \\
0 \\
0 \\
\vec{n}\end{array}$ & $\begin{array}{l}8 \\
8 \\
0 \\
0 \\
0 \\
0 \\
0 \\
n\end{array}$ \\
\hline$\infty$ & 0 & 0 & 0 & 0 & 0 & 0 & o & o & 0 & 0 & 0 \\
\hline$<$ & $\begin{array}{l}\Xi \\
\vdots \\
\square\end{array}$ & $\begin{array}{l}\vec{\Xi} \\
\dot{0} \\
\dot{0}\end{array}$ & $\begin{array}{l}\hat{0} \\
\vdots \\
0\end{array}$ & $\begin{array}{l}\vec{E} \\
\dot{0} \\
\dot{0}\end{array}$ & $\begin{array}{l}\vec{I} \\
\dot{E}\end{array}$ & $\vec{\Xi}$ & $\begin{array}{l}= \\
\vdots \\
\vdots\end{array}$ & $\begin{array}{l}\square \\
\vdots \\
\vdots\end{array}$ & $\begin{array}{l} \pm \\
\vdots \\
\vdots\end{array}$ & $\begin{array}{l}\Xi \\
\Xi \\
\vdots\end{array}$ & $\begin{array}{l}= \\
\square \\
0\end{array}$ \\
\hline$\underset{\vdash}{Y}$ & $\begin{array}{l}8 \\
8 \\
0 \\
1 \\
0 \\
\text { in } \\
m\end{array}$ & $\begin{array}{l}\circ \\
8 \\
\infty \\
1 \\
1 \\
0 \\
\text { in } \\
m\end{array}$ & $\begin{array}{l}\stackrel{0}{n} \\
\stackrel{n}{n} \\
\vdots \\
0 \\
\text { nn }\end{array}$ & $\begin{array}{l}\text { 은 } \\
\text { î } \\
\text { o } \\
\text { n }\end{array}$ & 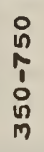 & $\begin{array}{l}8 \\
: \\
0 \\
0 \\
0 \\
0\end{array}$ & $\begin{array}{l}\text { : } \\
\text { h } \\
\text { 1 } \\
\text { o } \\
\text { ñ }\end{array}$ & $\begin{array}{l}8 \\
\text { : } \\
0 \\
\text { o } \\
\text { in }\end{array}$ & $\begin{array}{l}8 \\
\text { in } \\
1 \\
\text { o } \\
\text { ñ }\end{array}$ & $\begin{array}{l}8 \\
0 \\
1 \\
1 \\
0 \\
m\end{array}$ & $\begin{array}{l}8 \\
1 \\
8 \\
8 \\
8 \\
0\end{array}$ \\
\hline
\end{tabular}

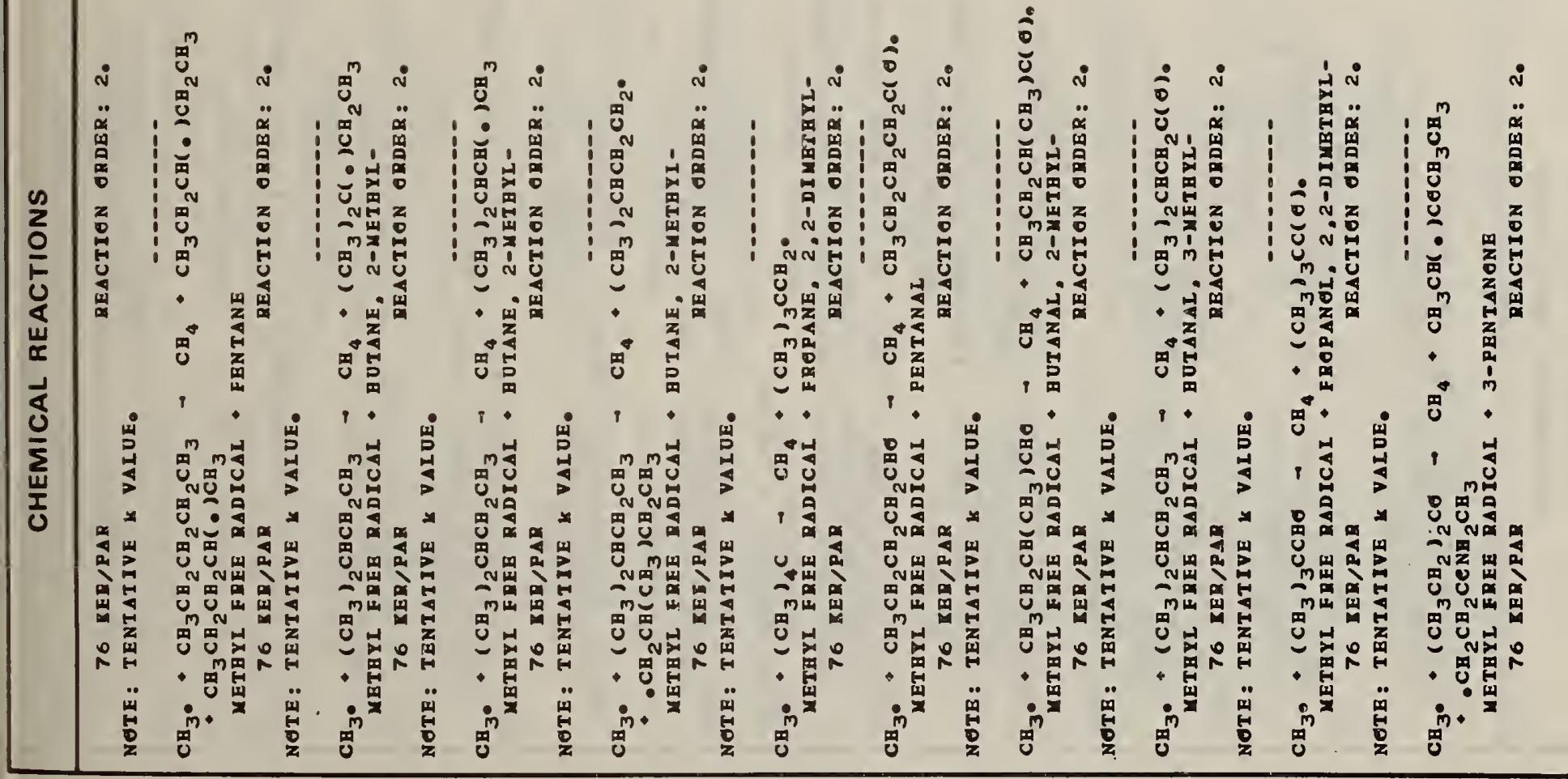




\begin{tabular}{|c|c|c|c|c|c|c|c|c|c|c|c|}
\hline $\begin{array}{l}0^{4} \\
0 \\
0 \\
0 \\
\vdots \\
x-\end{array}$ & $\begin{array}{l}: \\
\text { i } \\
:\end{array}$ & $\begin{array}{l}: \\
\therefore \\
?\end{array}$ & $\begin{array}{l}: \\
\text { N } \\
?\end{array}$ & $\begin{array}{l}? \\
: \\
?\end{array}$ & $\begin{array}{l}: \\
\text { i } \\
\text { : }\end{array}$ & $\begin{array}{l}? \\
: \\
:\end{array}$ & & & & & $\begin{array}{l}: \\
\therefore\end{array}$ \\
\hline 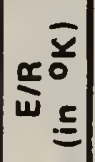 & 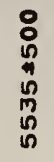 & \begin{tabular}{l}
$:$ \\
$:$ \\
$:$ \\
$:$ \\
$:$ \\
$\stackrel{0}{0}$ \\
\hdashline
\end{tabular} & 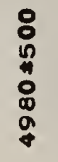 & $\begin{array}{l}: \\
: \\
0 \\
\stackrel{n}{N} \\
\stackrel{0}{+}\end{array}$ & ' & $\begin{array}{l}: \\
0 \\
0 \\
0 \\
0 \\
\vdots \\
n \\
n\end{array}$ & $:$ & 昌 & $\begin{array}{l}\stackrel{\circ}{0} \\
\stackrel{n}{m}\end{array}$ & 吕 & $\begin{array}{l}: \\
: \\
: \\
: \\
\vdots \\
\end{array}$ \\
\hline$\infty$ & 0 & 0 & 0 & 0 & I & 。 & ' & I & ' & ' & 。 \\
\hline$\alpha$ & $\begin{array}{l}\bar{\Xi} \\
\dot{\vdots} \\
\dot{0}\end{array}$ & $\bar{\vdots}$ & $\underset{\vdots}{\vdots}$ & $\begin{array}{l}\bar{\vdots} \\
\vdots \\
\vdots\end{array}$ & $\grave{n}$ & $\begin{array}{l}\bar{\vdots} \\
\vdots \\
\dot{0}\end{array}$ & ' & ' & ' & ' & $\begin{array}{l}\bar{\Xi} \\
\dot{0} \\
\stackrel{0}{0}\end{array}$ \\
\hline & $\begin{array}{l}: \\
0 \\
\vdots \\
\vdots \\
\vdots\end{array}$ & $\begin{array}{l}: \\
: \\
\vdots \\
: \\
:\end{array}$ & $\begin{array}{l}: \\
0 \\
0 \\
0 \\
0 \\
n\end{array}$ & $\begin{array}{l}\text { : } \\
0 \\
\vdots \\
\vdots \\
\circ\end{array}$ & 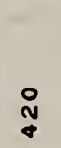 & $\begin{array}{l}: \\
\qquad \\
0 \\
1 \\
0 \\
n \\
m\end{array}$ & $\underset{\sim}{\infty}$ & $\begin{array}{l}\infty \\
\stackrel{\infty}{\alpha}\end{array}$ & $\begin{array}{c}\infty \\
\alpha \\
\alpha\end{array}$ & $\stackrel{\infty}{\stackrel{\infty}{N}}$ & 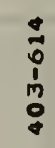 \\
\hline
\end{tabular}

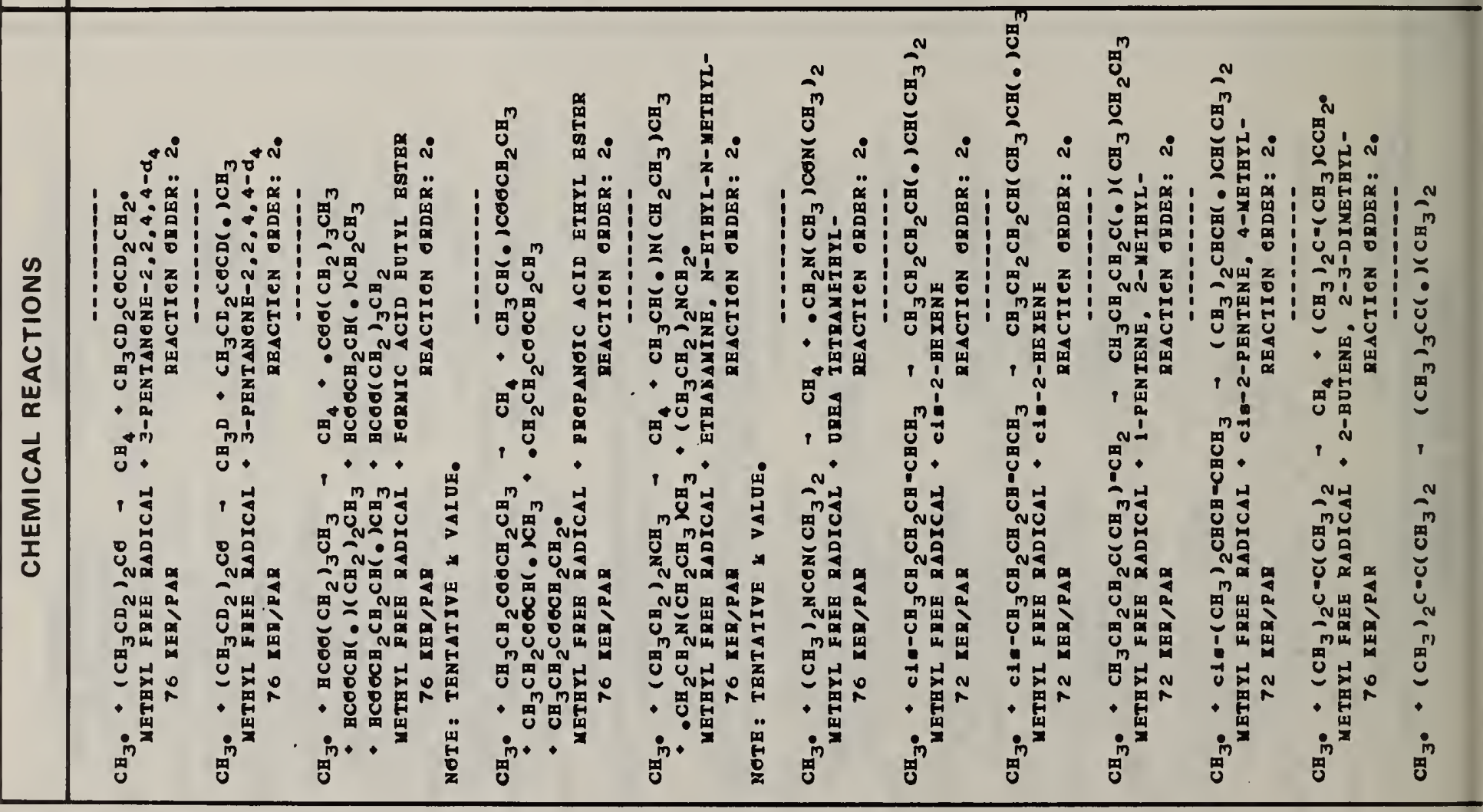




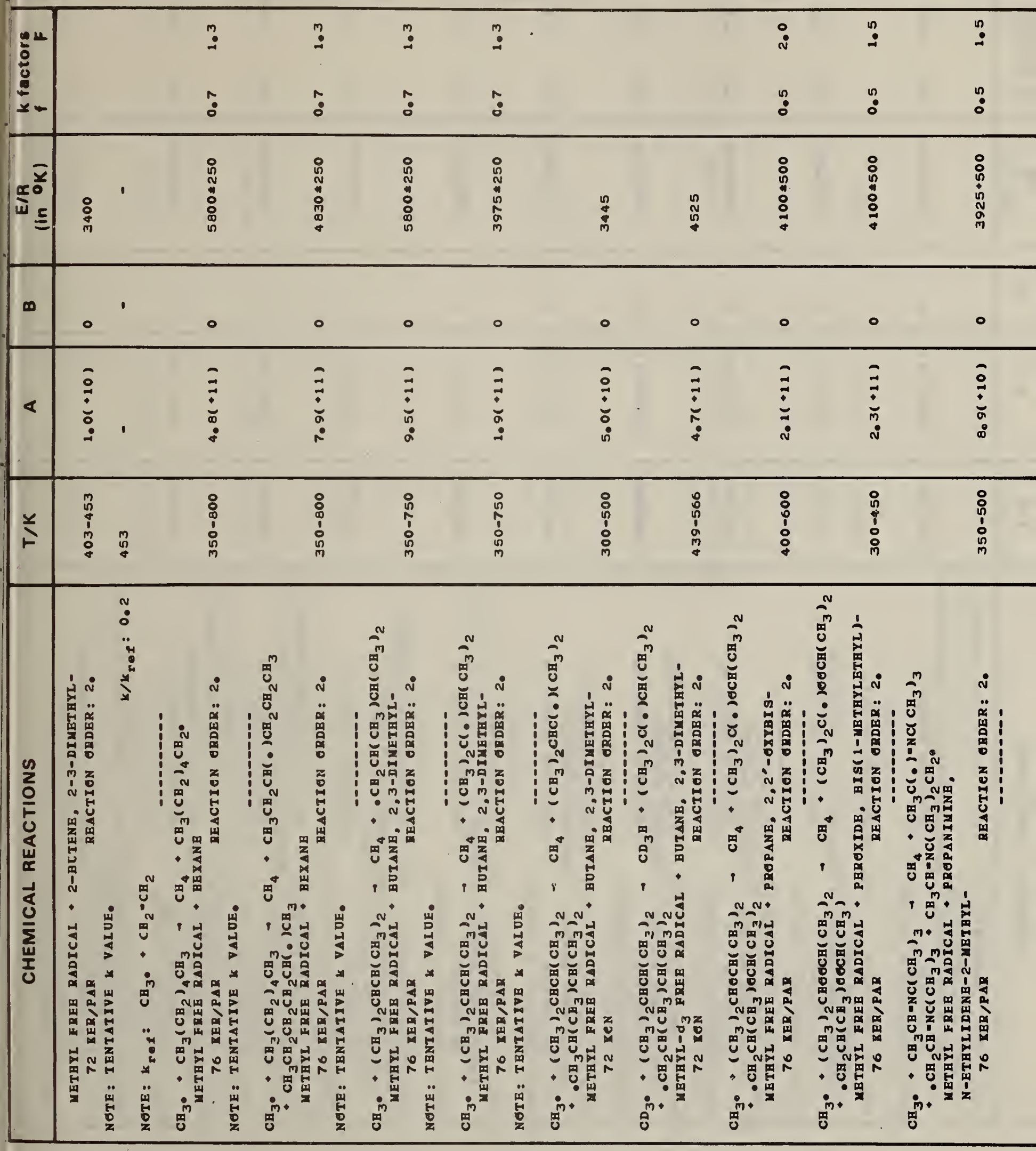




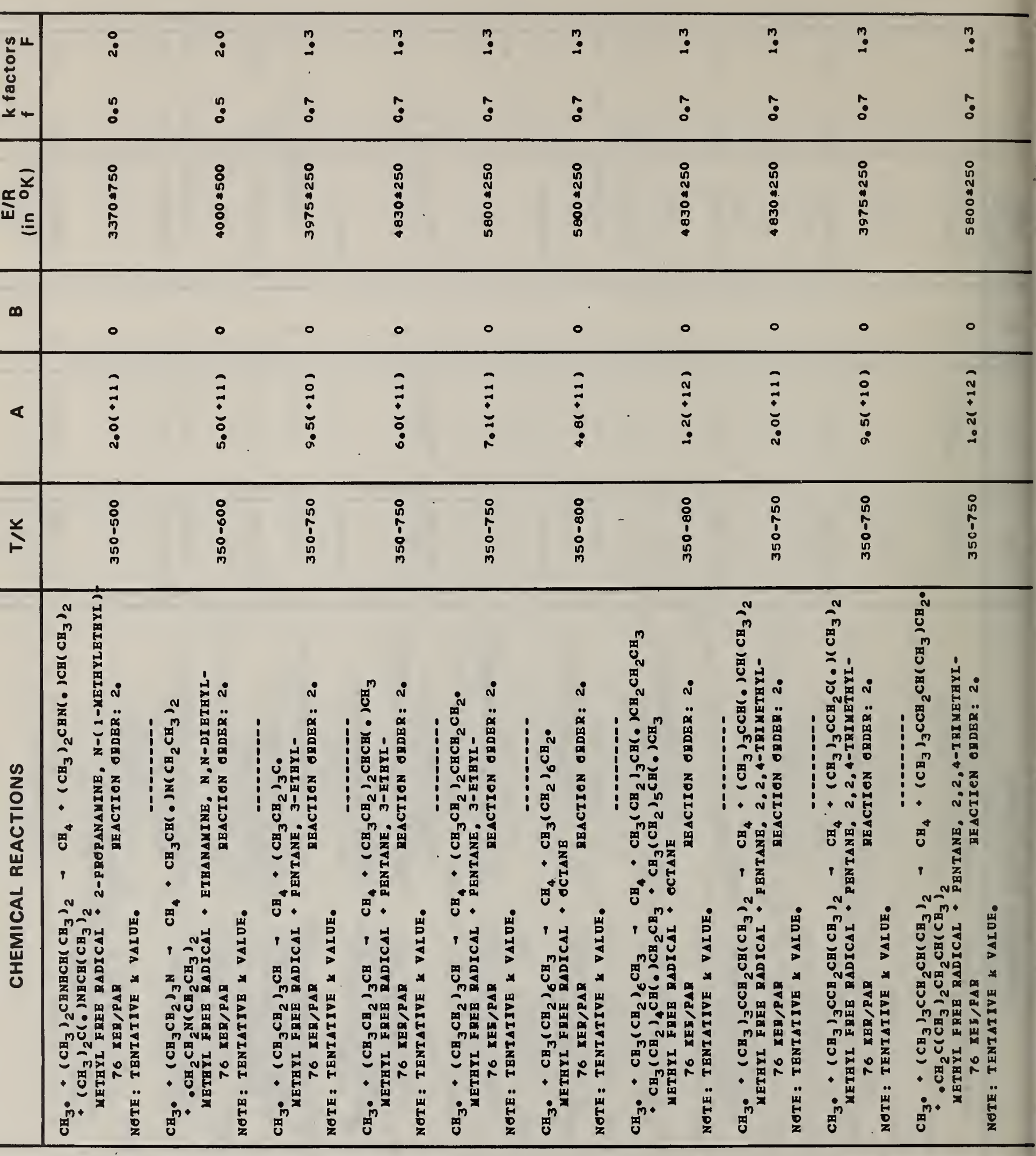




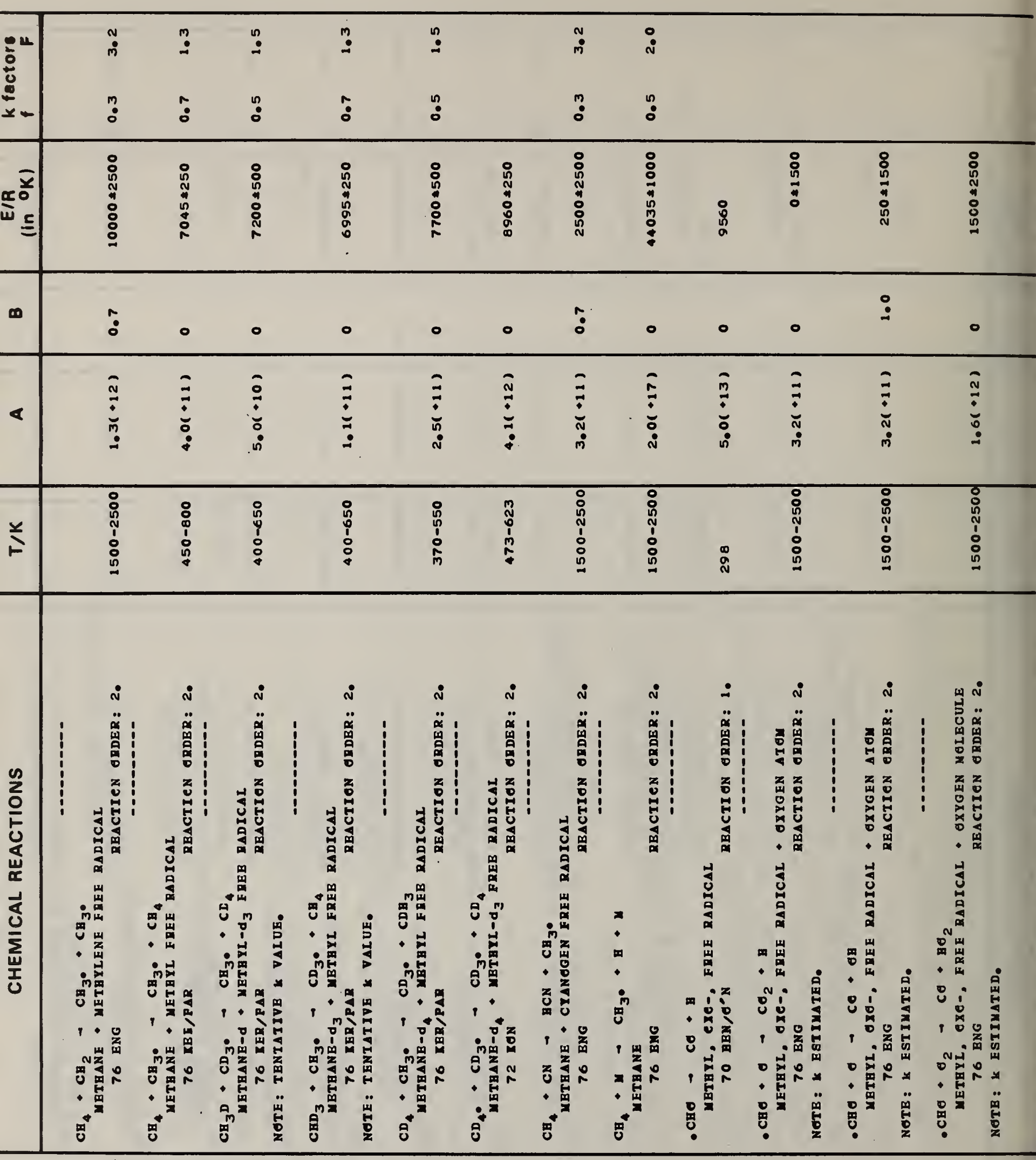




\begin{tabular}{|c|c|c|c|c|c|c|c|c|c|c|c|c|c|c|c|c|}
\hline $\begin{array}{l}2^{2} \\
0 \\
0 \\
0 \\
x \\
x\end{array}$ & $\begin{array}{l}N \\
\dot{m} \\
\dot{0}\end{array}$ & & & & & & & $\begin{array}{l}\stackrel{v}{m} \\
\dot{m}\end{array}$ & $\begin{array}{l}N \\
m \\
m \\
:\end{array}$ & $\begin{array}{l}N \\
\dot{m} \\
\dot{m}\end{array}$ & $\begin{array}{l}\text { N } \\
m \\
m\end{array}$ & $\begin{array}{l}\text { v } \\
\text { m } \\
?\end{array}$ & $\begin{array}{l}\text { m } \\
m \\
m\end{array}$ & $\begin{array}{l}N \\
\dot{m} \\
m \\
\dot{0}\end{array}$ & $\begin{array}{l}\text { s } \\
m \\
m\end{array}$ & \\
\hline$\alpha^{\frac{z}{0}}=$ & $\begin{array}{l}\text { : } \\
\text { 吕 } \\
\text { N } \\
\text { ڤै }\end{array}$ & 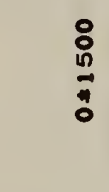 & & $\begin{array}{l}8 \\
: \\
10 \\
0 \\
: \\
:\end{array}$ & & $\begin{array}{l}8 \\
\stackrel{8}{0} \\
0\end{array}$ & & $\begin{array}{l}8 \\
0 \\
0 \\
1 \\
0 \\
0 \\
8 \\
0\end{array}$ & $\begin{array}{l}8 \\
0 \\
N \\
1 \\
0 \\
0 \\
0\end{array}$ & 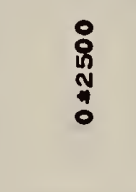 & 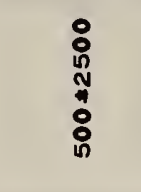 & $\begin{array}{l}: \\
: \\
\stackrel{n}{N} \\
: \\
: \\
:\end{array}$ & $\begin{array}{l}8 \\
\text { ํㅜ } \\
\text { N } \\
\text { pै }\end{array}$ & $\begin{array}{l}: \\
\stackrel{0}{N} \\
\text { : } \\
\text { :ै }\end{array}$ & $\begin{array}{l}\stackrel{0}{0} \\
\text { h } \\
\text { N } \\
0 \\
0\end{array}$ & \\
\hline$\infty$ & ? & : & & 0 & & 0 & & ? & $\because$ & ?: & $\because$ & $\because$ & $\stackrel{10}{:}$ & ? & : & \\
\hline$<$ & $\begin{array}{l}\hat{\Xi} \\
\vdots \\
0\end{array}$ & $\begin{array}{l}\hat{O} \\
\vdots \\
\text { N } \\
\dot{m}\end{array}$ & & $\begin{array}{l} \pm \\
\vdots \\
0\end{array}$ & & $\stackrel{\square}{\square}$ & & $\begin{array}{l}\overrightarrow{0} \\
\dot{0} \\
\dot{0}\end{array}$ & $\begin{array}{l}\vec{\vdots} \\
\dot{0} \\
\dot{0}\end{array}$ & $\begin{array}{l}\vec{a} \\
\dot{v} \\
\dot{m}\end{array}$ & $\begin{array}{l}0 \\
\stackrel{0}{0} \\
\dot{n} \\
\dot{m}\end{array}$ & $\begin{array}{l}\hat{0} \\
\dot{n} \\
\dot{m}\end{array}$ & $\begin{array}{l}= \\
\bar{N} \\
\dot{0}\end{array}$ & $\begin{array}{l}\vdots \\
\vdots \\
\vdots\end{array}$ & $\begin{array}{l}= \\
\vdots \\
\vdots\end{array}$ & \\
\hline$\stackrel{Y}{F}$ & $\begin{array}{l}\circ \\
: \\
0 \\
N \\
1 \\
0 \\
0 \\
\vdots\end{array}$ & 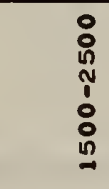 & & 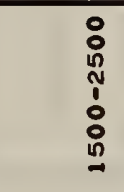 & & 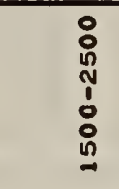 & & $\begin{array}{l}: \\
8 \\
\stackrel{8}{1} \\
\vdots \\
: \\
:\end{array}$ & $\begin{array}{l}: \\
8 \\
10 \\
1 \\
0 \\
: \\
0 \\
0\end{array}$ & \begin{tabular}{l}
$:$ \\
$:$ \\
$\stackrel{8}{N}$ \\
$\vdots$ \\
$:$ \\
$:$ \\
\hdashline
\end{tabular} & $\begin{array}{l}0 \\
: \\
\stackrel{0}{n} \\
\vdots \\
\vdots \\
:\end{array}$ & 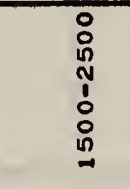 & \begin{tabular}{l}
$:$ \\
$:$ \\
$\stackrel{8}{n}$ \\
1 \\
$:$ \\
$:$ \\
\hdashline
\end{tabular} & $\begin{array}{l}\text { : } \\
\text { in } \\
\vdots \\
\vdots \\
\vdots\end{array}$ & 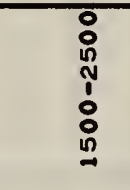 & \\
\hline 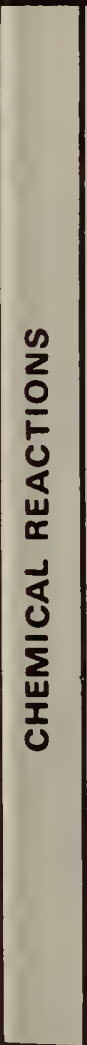 & 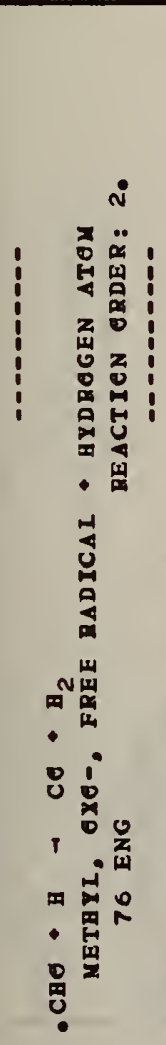 & 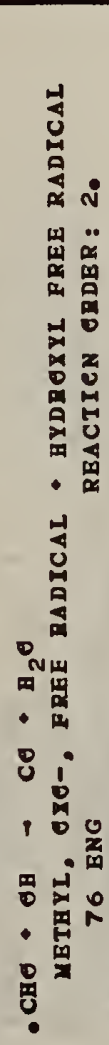 & 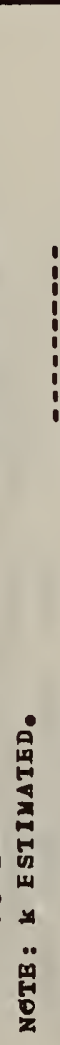 & 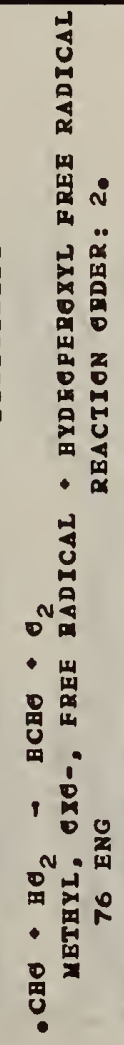 & 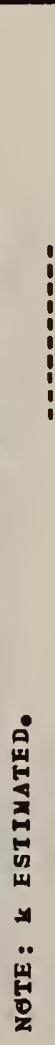 & 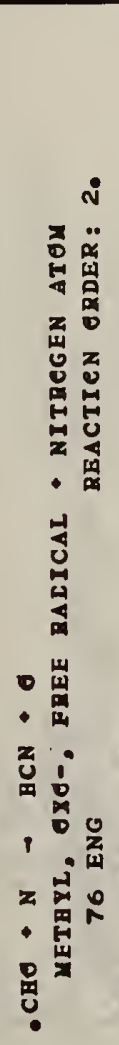 & 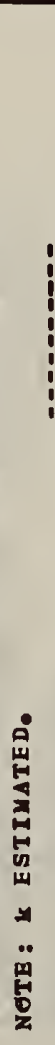 & 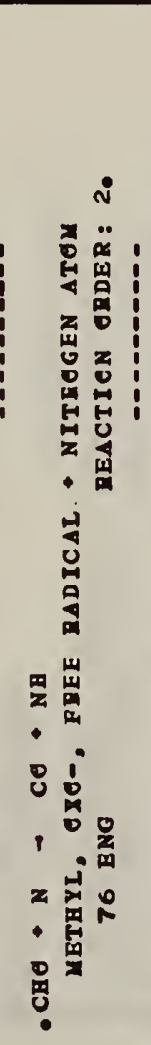 & 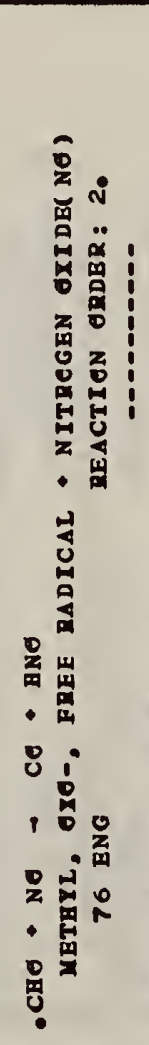 & 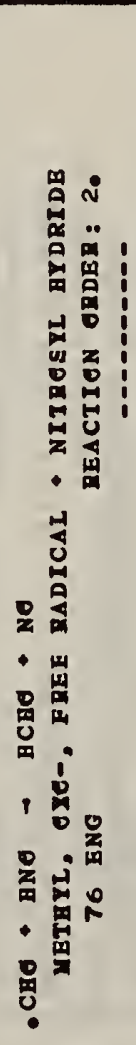 & 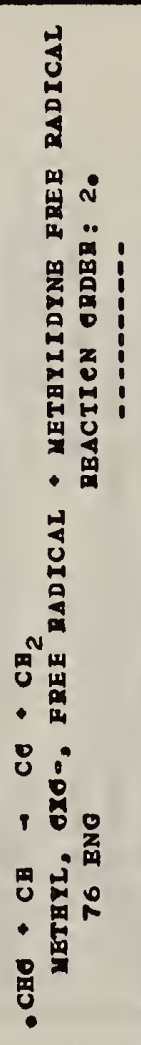 & 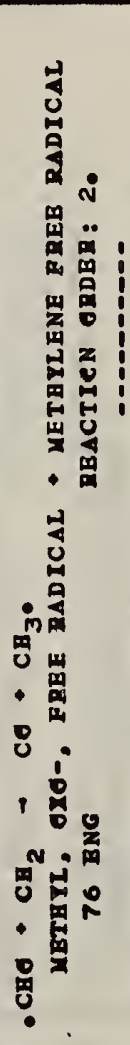 & 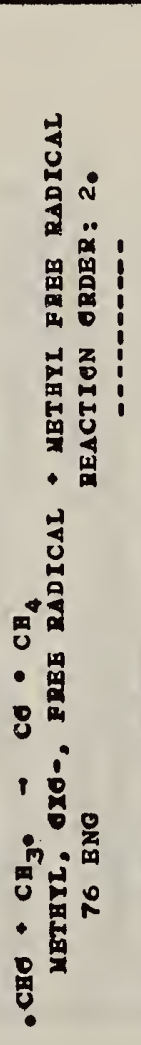 & 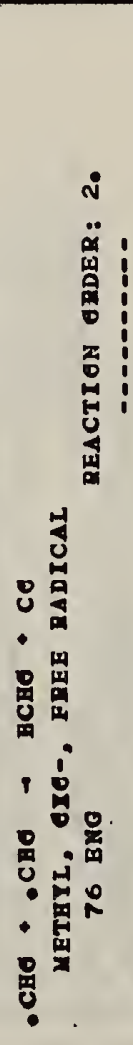 & 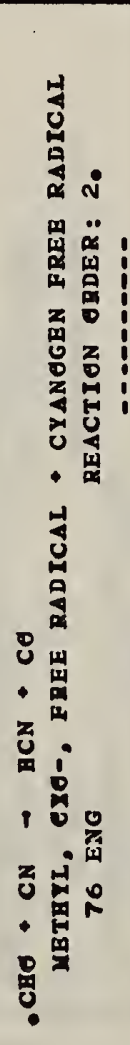 & 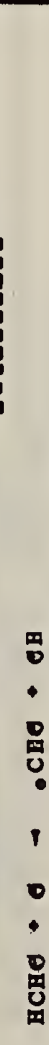 \\
\hline
\end{tabular}




\begin{tabular}{|c|c|c|c|c|c|c|c|c|c|c|c|}
\hline $\begin{array}{l}0^{\prime \prime} \\
0 \\
0 \\
0 \\
x \\
x-2\end{array}$ & . & & & & & & $\begin{array}{l}: \\
:\end{array}$ & $\begin{array}{l}: \\
:\end{array}$ & $\begin{array}{l}: \\
:\end{array}$ & $\begin{array}{l}: \\
\text { N } \\
:\end{array}$ & $\begin{array}{l}: \\
\text { i } \\
:\end{array}$ \\
\hline 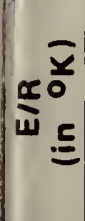 & $\begin{array}{l}: \\
\stackrel{\circ}{0} \\
\stackrel{0}{0}\end{array}$ & $\begin{array}{l}: \\
: \\
: \\
: \\
:\end{array}$ & $\begin{array}{l}: \\
\stackrel{8}{0} \\
0 \\
0\end{array}$ & : & $\stackrel{\circ}{:}$ & 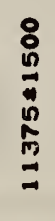 & $\begin{array}{l}8 \\
8 \\
0 \\
00 \\
0 \\
0 \\
0\end{array}$ & 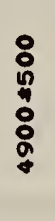 & 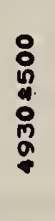 & $\begin{array}{l}: \\
: \\
0 \\
: \\
: \\
: \\
0\end{array}$ & $\begin{array}{l}: \\
: \\
: \\
: \\
\vdots \\
0 \\
0\end{array}$ \\
\hline$\infty$ & 0 & 0 & 0 & 0 & 0 & $\stackrel{n}{\because}$ & 0 & 0 & 0 & 0 & 0 \\
\hline$<$ & $\begin{array}{l}\dot{\vdots} \\
\dot{\vdots}\end{array}$ & $\stackrel{\tilde{N}}{\stackrel{0}{0}}$ & $\dot{\vdots}$ & $\underset{\dot{n}}{\tilde{n}}$ & 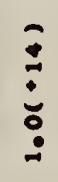 & $\begin{array}{l}\hat{0} \\
\vdots \\
\vdots\end{array}$ & $\begin{array}{l}\Xi \\
\Xi \\
\vdots\end{array}$ & $\begin{array}{l}\hat{0} \\
\vdots \\
\vdots \\
\vdots\end{array}$ & $\begin{array}{l}\bar{z} \\
\dot{n} \\
\dot{n}\end{array}$ & $\stackrel{\square}{\vdots}$ & 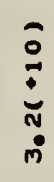 \\
\hline Y & $\begin{array}{l}\text { : } \\
\stackrel{్}{n} \\
\vdots \\
: \\
\stackrel{0}{n}\end{array}$ & $\begin{array}{l}0 \\
: \\
\stackrel{\circ}{n} \\
\vdots \\
: \\
0 \\
0\end{array}$ & $\begin{array}{l}\text { : } \\
\stackrel{\$}{1} \\
\vdots \\
: \\
:\end{array}$ & 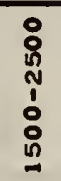 & 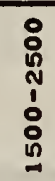 & 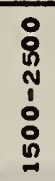 & $\begin{array}{l}\circ \\
8 \\
1 \\
1 \\
\circ \\
\text { in } \\
m\end{array}$ & & 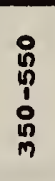 & $\begin{array}{l}: \\
0 \\
! \\
\vdots \\
\vdots\end{array}$ & $\begin{array}{l}: \\
! \\
1 \\
:\end{array}$ \\
\hline
\end{tabular}

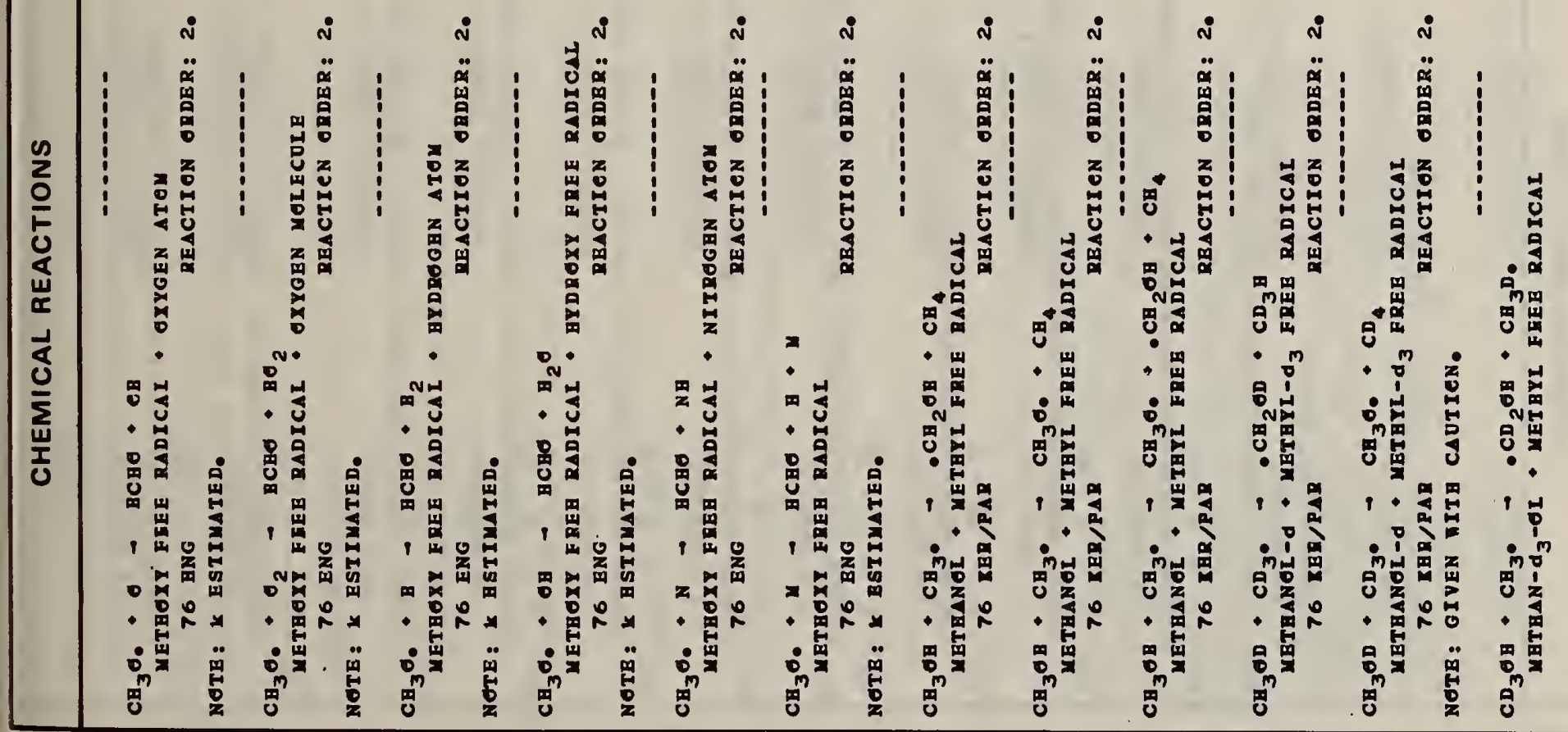




\begin{tabular}{|c|c|c|c|c|c|c|c|c|c|c|c|c|c|}
\hline 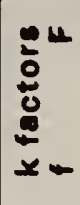 & & $\begin{array}{l}\vdots \\
:\end{array}$ & & $\begin{array}{l}\stackrel{0}{=} \\
\stackrel{0}{\circ}\end{array}$ & & & & & & & $\begin{array}{l}\stackrel{n}{0} \\
\stackrel{0}{0}\end{array}$ & & \\
\hline 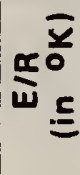 & $\mid \begin{array}{l}0 \\
0 \\
0 \\
0 \\
0 \\
0 \\
0 \\
0 \\
0\end{array}$ & $\begin{array}{l}\stackrel{0}{0} \\
0 \\
\vdots \\
\vdots \\
\vdots\end{array}$ & 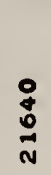 & ' & 0 & 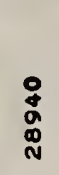 & $\begin{array}{l}\text { 易 } \\
\text { : }\end{array}$ & $\begin{array}{l}\stackrel{0}{\mathbf{w}} \\
\underset{\varpi}{+}\end{array}$ & 0 & $\begin{array}{c}n \\
0 \\
\vdots \\
\vdots \\
\vdots\end{array}$ & 号 & $\stackrel{8}{\Xi}$ & $\begin{array}{l}\stackrel{8}{\mathrm{~N}} \\
\stackrel{n}{n}\end{array}$ \\
\hline$\infty$ & 0 & 0 & ० & ' & $\stackrel{n}{0}$ & $\stackrel{n}{0}$ & $\stackrel{\text { : }}{0}$ & $\stackrel{n}{0}$ & $\stackrel{0}{0}$ & $\stackrel{n}{0}$ & 。 & $\stackrel{?}{0}$ & $\stackrel{0}{0}$ \\
\hline$<$ & ס & : & $\begin{array}{l}\vdots \\
\vdots \\
\vdots \\
\vdots\end{array}$ & 孞 & $\bar{\vdots}$ & $\underset{\mathfrak{N}}{\mathfrak{b}}$ & $\stackrel{\bar{n}}{\vdots}$ & $\begin{array}{l}m \\
\vdots \\
\dot{0}\end{array}$ & $\begin{array}{l}\bar{\vdots} \\
\dot{m} \\
\dot{0}\end{array}$ & $\stackrel{\hat{N}}{\square}$ & & $\stackrel{\widehat{N}}{\underline{m}}$ & $\begin{array}{l}\bar{N} \\
\vdots \\
\vdots \\
\vdots\end{array}$ \\
\hline 兰 & $\begin{array}{l}0 \\
0 \\
0 \\
0 \\
0 \\
i \\
n\end{array}$ & $\begin{array}{l}0 \\
0 \\
0 \\
\vdots \\
\vdots \\
n\end{array}$ & 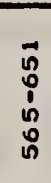 & י & & & & & & & 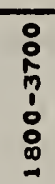 & & \\
\hline
\end{tabular}

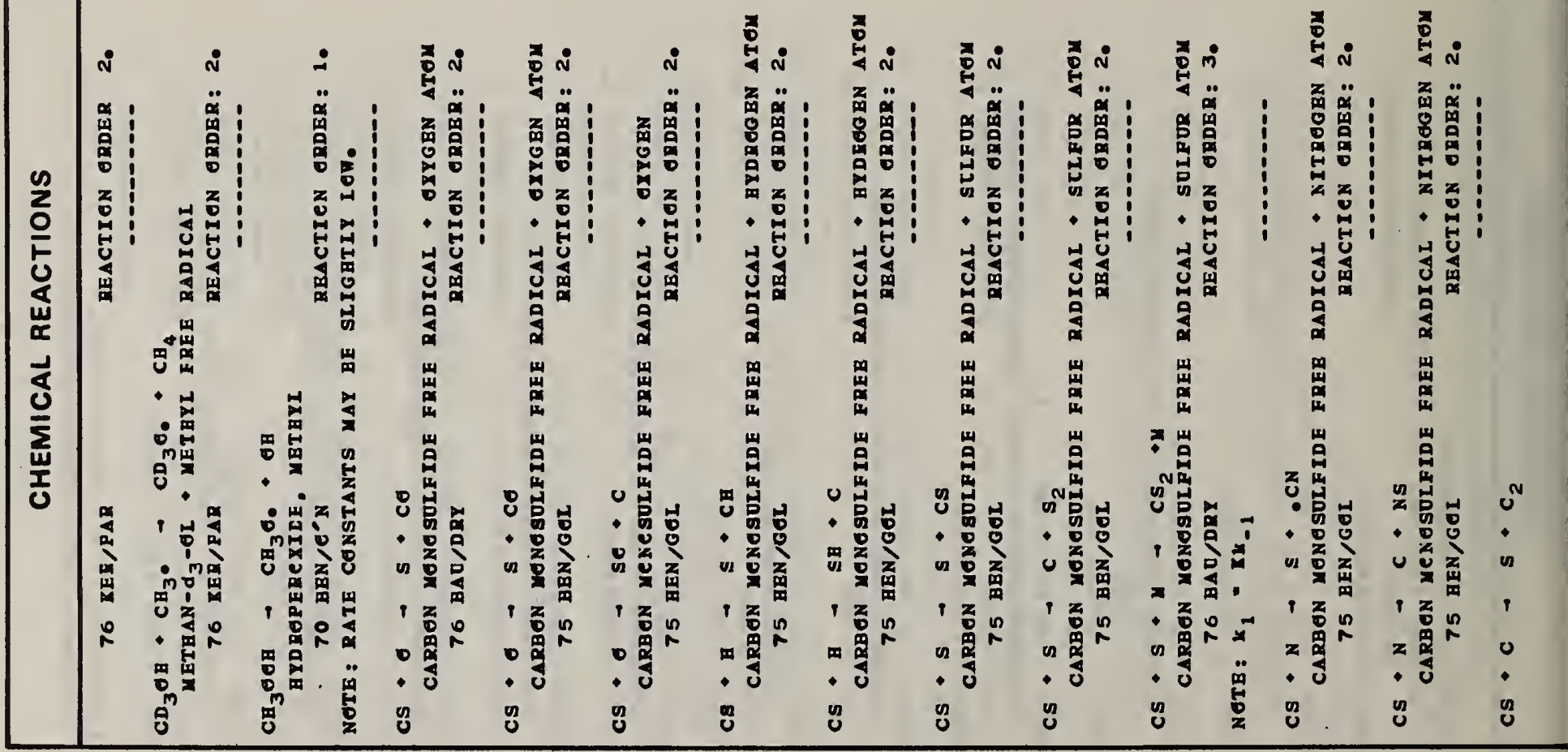




\begin{tabular}{|c|c|c|c|c|c|c|c|c|c|c|c|c|c|}
\hline 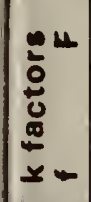 & & & $\begin{array}{l}? \\
:\end{array}$ & $\begin{array}{l}n \\
: \\
? \\
:\end{array}$ & $\begin{array}{l}? \\
: \\
:\end{array}$ & $\begin{array}{l}\text { !n } \\
:\end{array}$ & $\begin{array}{l}\stackrel{?}{:} \\
:\end{array}$ & $\begin{array}{l}\text { ! } \\
: \\
:\end{array}$ & $\begin{array}{l}\dot{0} \\
\dot{m} \\
\ddot{0} \\
\ddot{0}\end{array}$ & & & & \\
\hline$\underbrace{\underline{\underline{w}}} \underline{\underline{z}}$ & $\begin{array}{l}\text { n } \\
\text { m } \\
\text { D } \\
\text { D }\end{array}$ & 0 & : & ' & 1 & 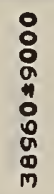 & $\begin{array}{l}\stackrel{B}{N} \\
\text { D. } \\
\stackrel{\leftrightarrow}{n} \\
\text { N }\end{array}$ & ' & 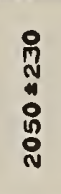 & I & ' & 1 & 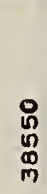 \\
\hline
\end{tabular}

$\infty$ : :

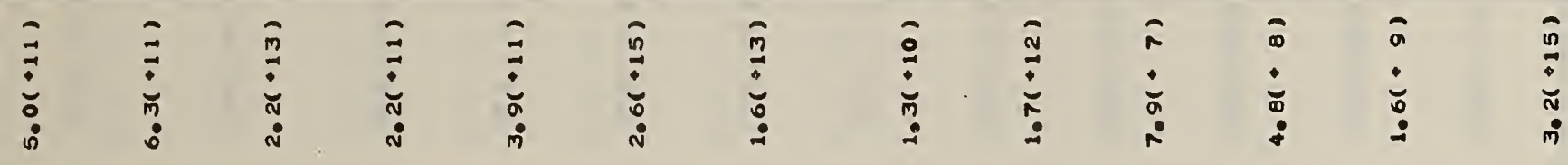

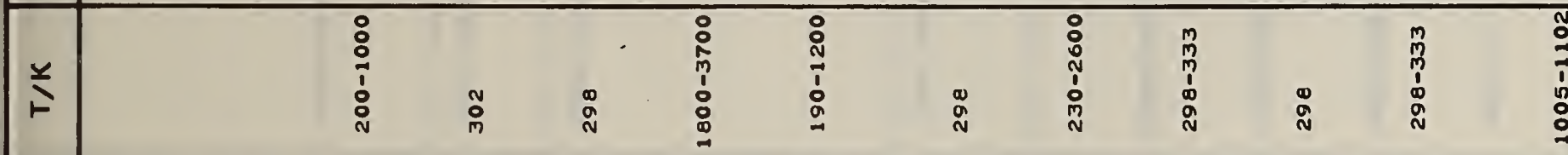

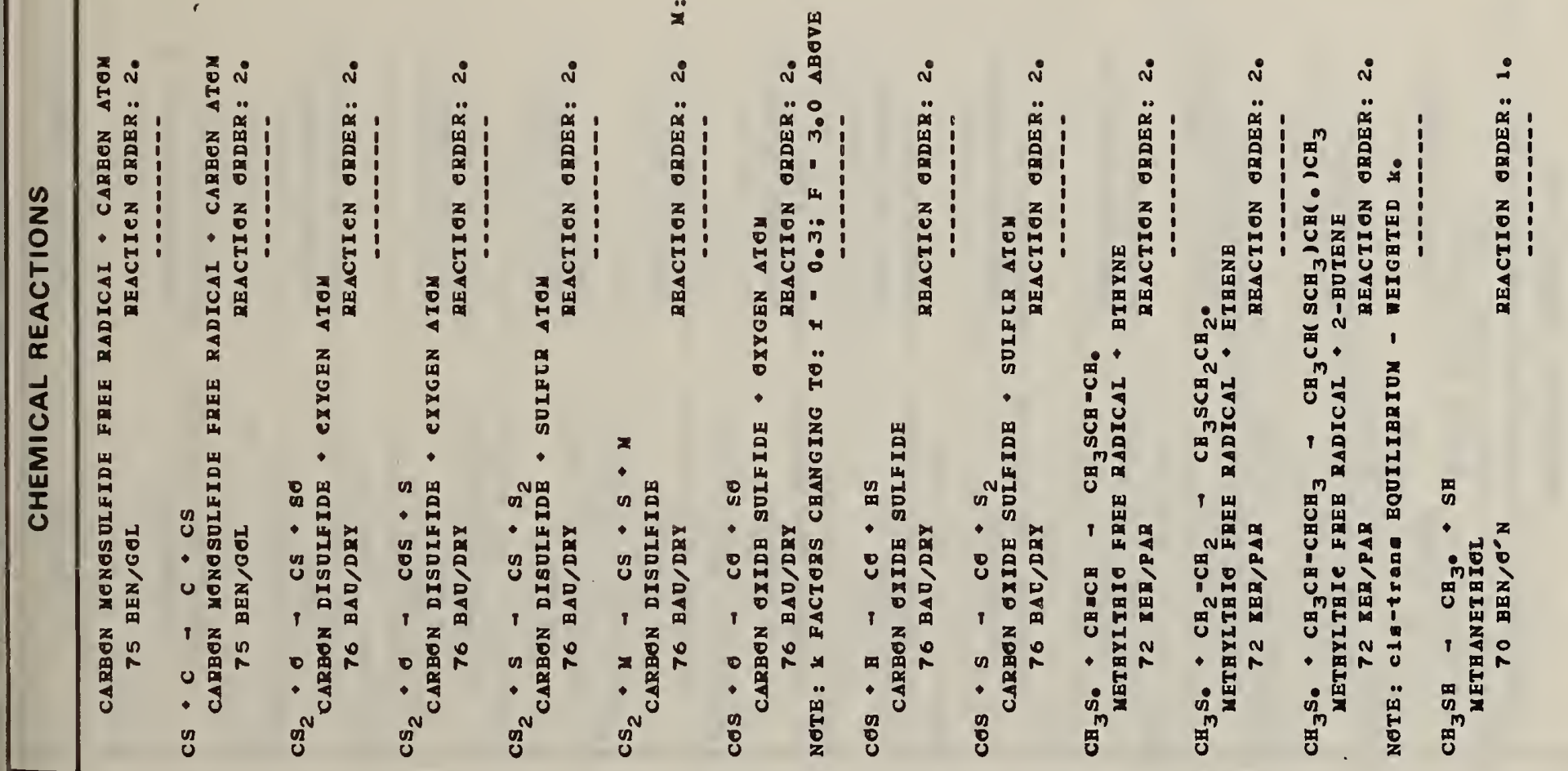




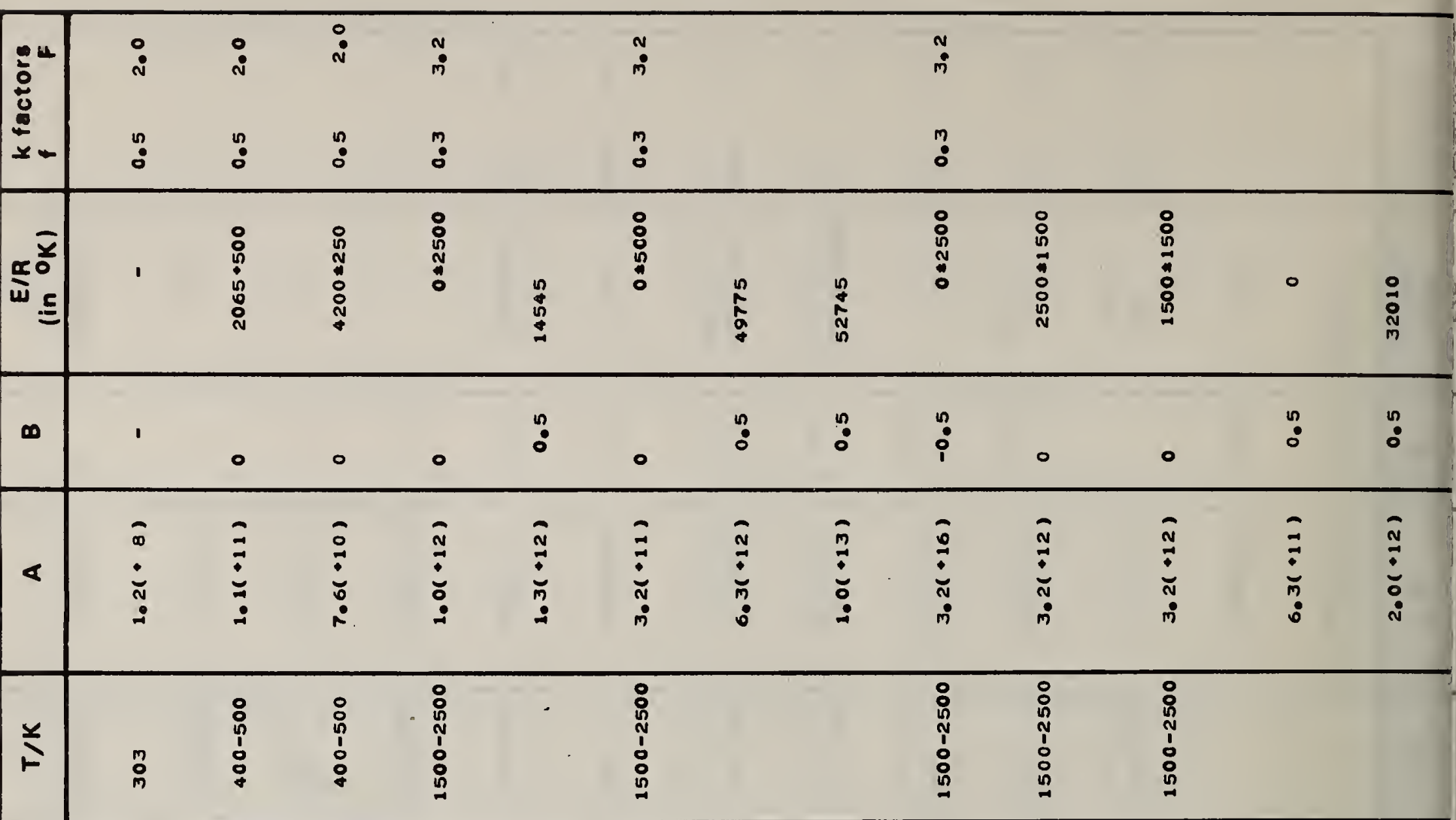

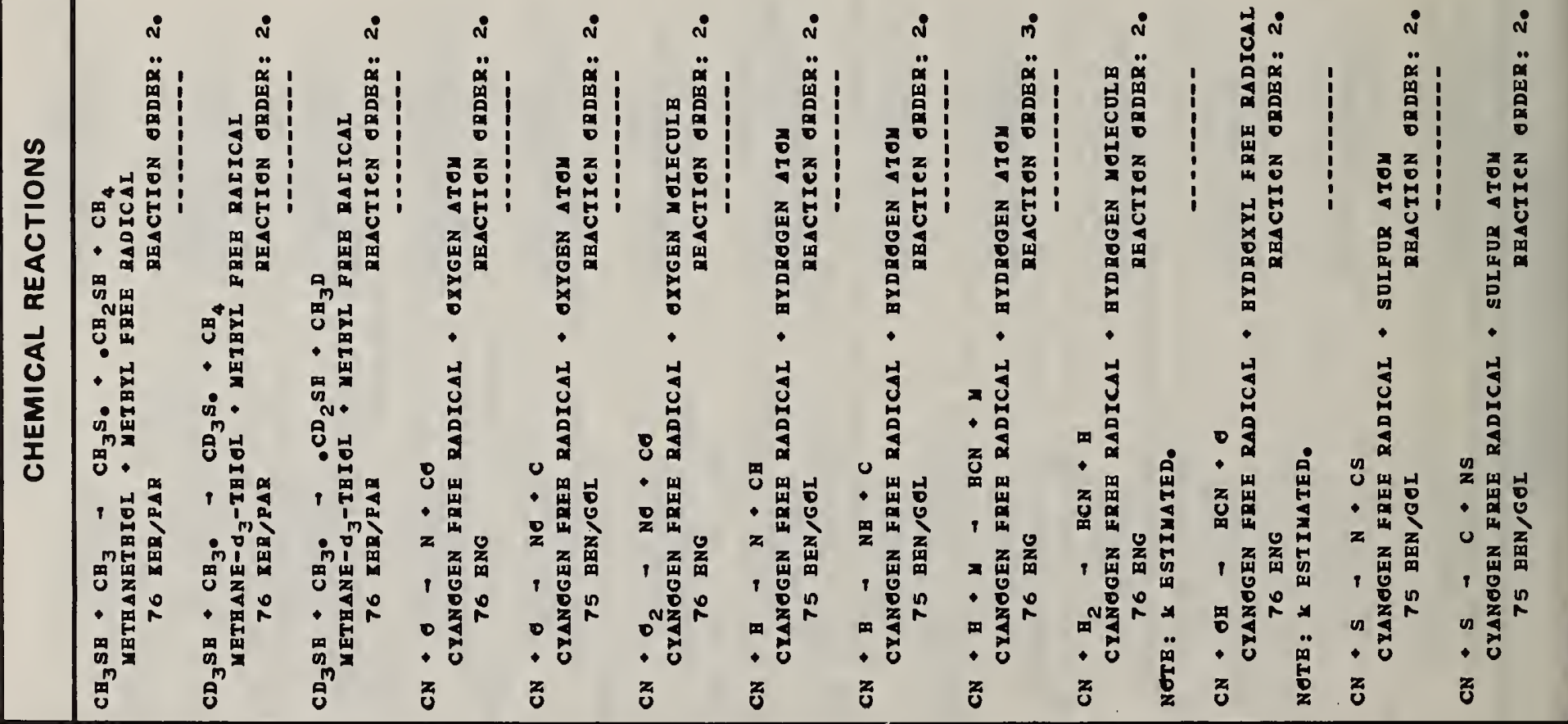




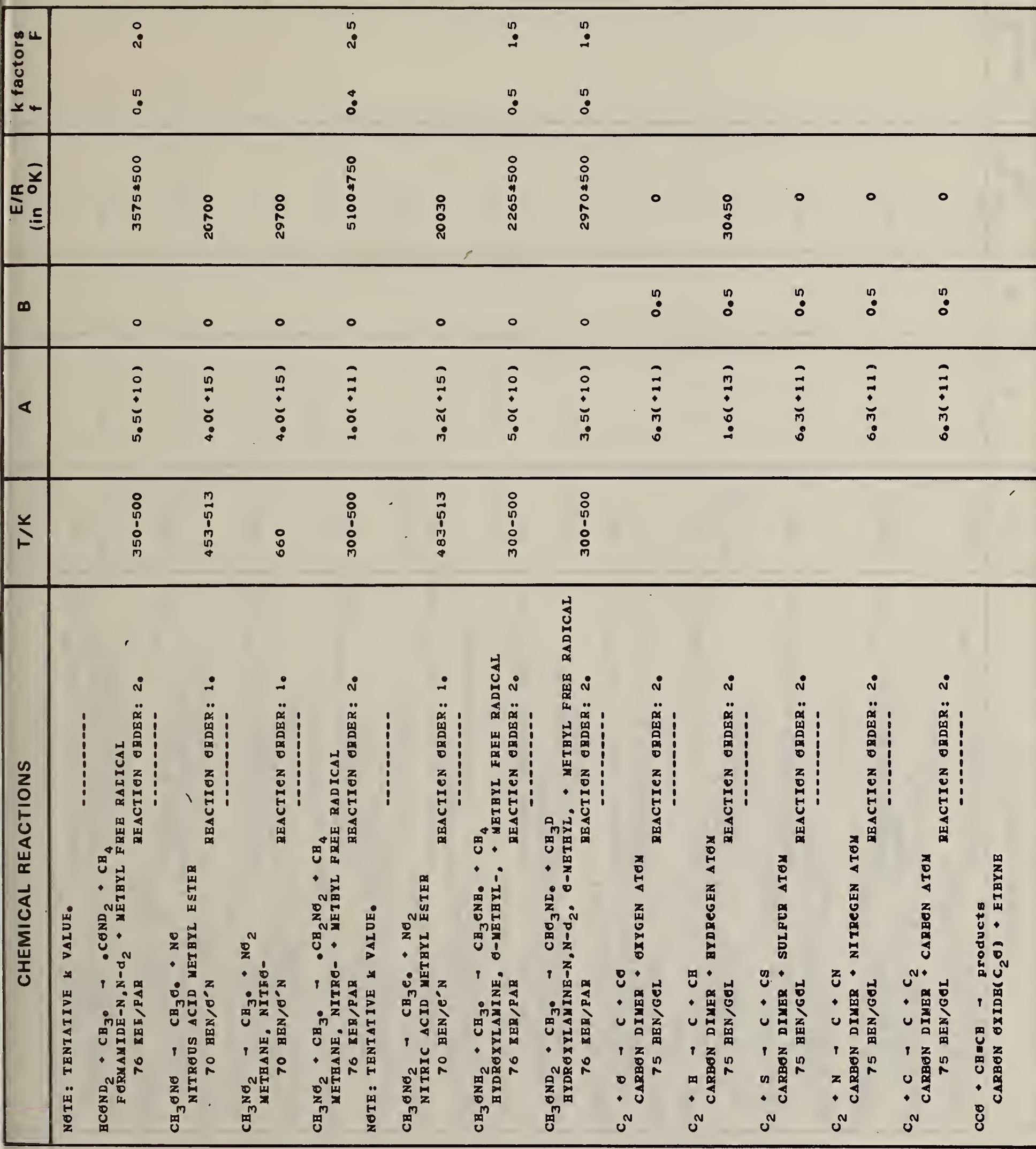




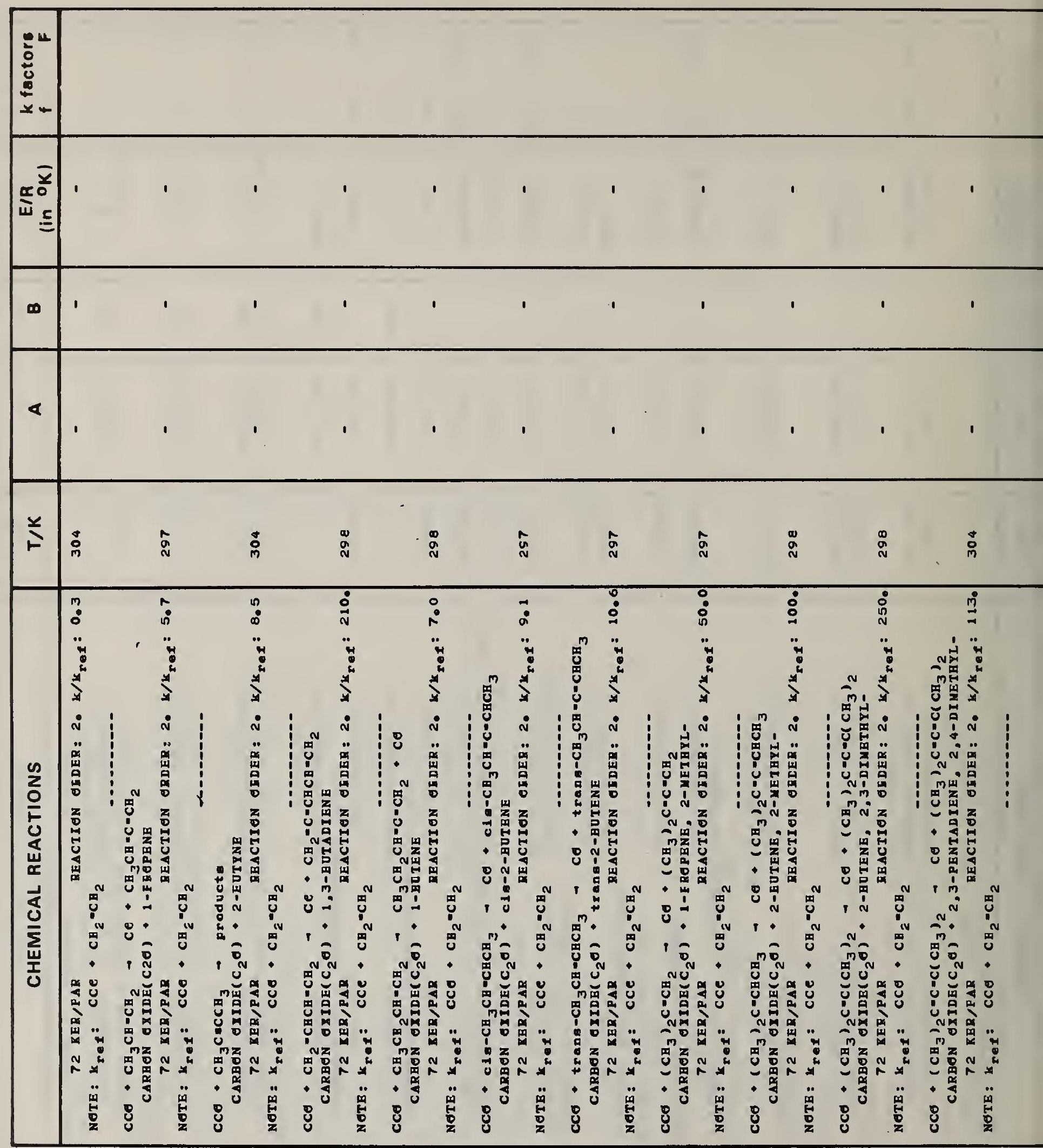




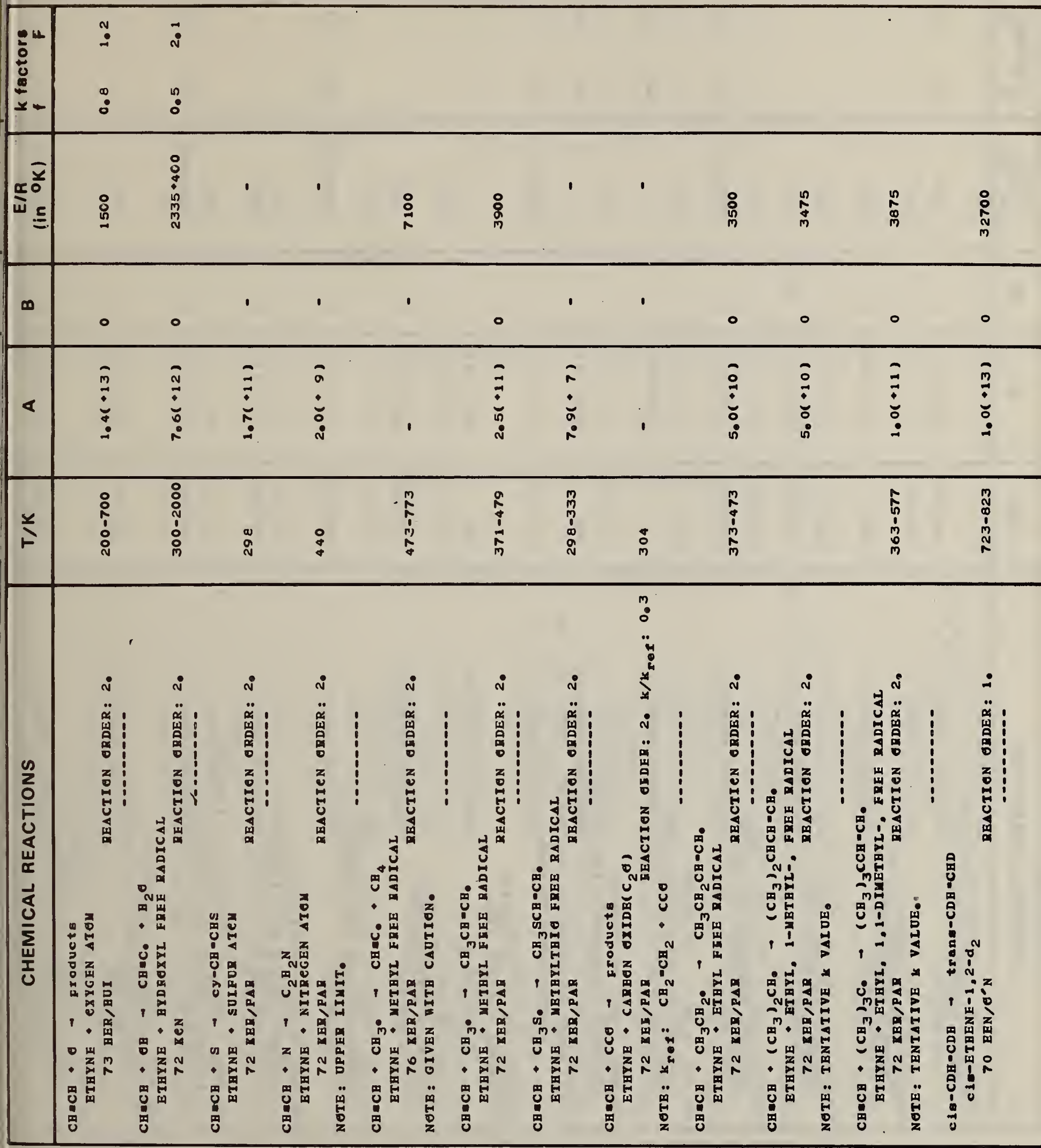




\begin{tabular}{|c|c|c|c|c|c|c|c|c|c|c|c|c|c|}
\hline 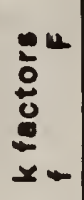 & $\begin{array}{l}\text { N. } \\
: \\
:\end{array}$ & & & $\begin{array}{l}\dot{s} \\
\dot{0}\end{array}$ & $\begin{array}{l}\text { m: } \\
\stackrel{\infty}{0}\end{array}$ & $\begin{array}{l}\dot{0} \\
\ddot{0}\end{array}$ & & & $\begin{array}{l}\stackrel{n}{-} \\
\stackrel{n}{0}\end{array}$ & & $\begin{array}{l}m \\
:\end{array}$ & & \\
\hline$\underbrace{\underline{\underline{x}}}$ & $\begin{array}{l}\text { ถึ } \\
\text { in }\end{array}$ & $\stackrel{0}{\square}$ & $\stackrel{\text { gn }}{+}$ & 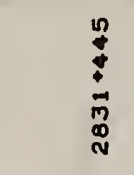 & ' & , & ' & 甍 & $\begin{array}{l}: \\
: \\
1 \\
: \\
: \\
:\end{array}$ & 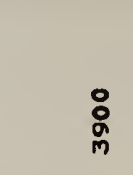 & $\begin{array}{l}\frac{n}{0} \\
\frac{7}{4} \\
n \\
n \\
n \\
n\end{array}$ & है & $\begin{array}{l}\stackrel{n}{0} \\
0 \\
m\end{array}$ \\
\hline $\boldsymbol{\infty}$ & 0 & 。 & $\stackrel{n}{0}$ & ० & I & ' & ' & - & ○ & - & 。 & ' & - \\
\hline$\varangle$ & 辡 & 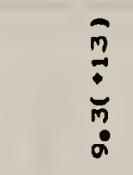 & $\begin{array}{l}\tilde{1} \\
\vdots \\
\vdots \\
0\end{array}$ & $\vdots$ & $\stackrel{N}{\vdots}$ & $\grave{n}$ & 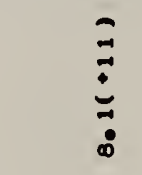 & $\begin{array}{l}0 \\
\vdots \\
\vdots \\
\dot{0}\end{array}$ & $\underset{\vdots}{\vdots}$ & $\underset{\vdots}{\vdots}$ & $\begin{array}{l}\vdots \\
\dot{0} \\
\dot{0}\end{array}$ & $\begin{array}{l}\tilde{\infty} \\
\dot{\varpi} \\
\dot{+}\end{array}$ & 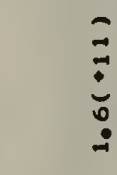 \\
\hline$\stackrel{y}{F}$ & 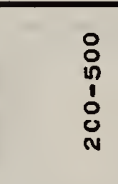 & $\stackrel{\infty}{\infty}$ & 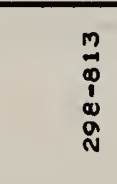 & $\begin{array}{l}: \\
\vdots \\
\vdots \\
\vdots \\
\vdots \\
⿱ ⺊ 口 \\
n\end{array}$ & . & $\stackrel{\circ}{\circ}$ & $\stackrel{\infty}{\stackrel{\infty}{N}}$ & 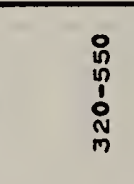 & 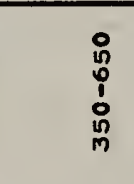 & 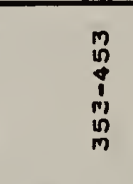 & 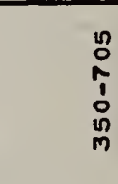 & $\stackrel{\Phi}{\text { ू }}$ & 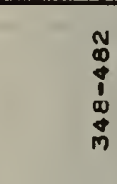 \\
\hline 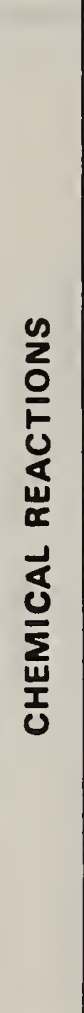 & 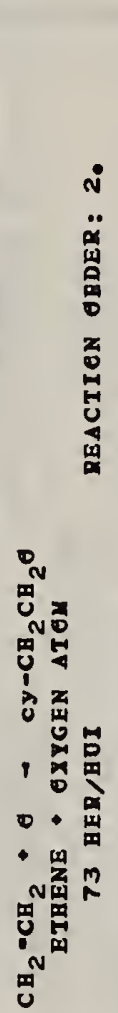 & 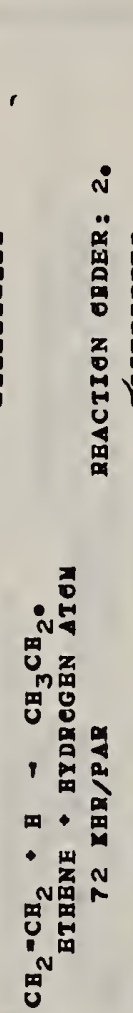 & 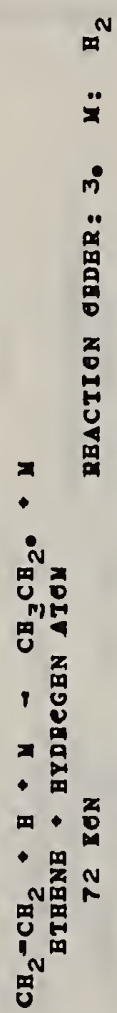 & 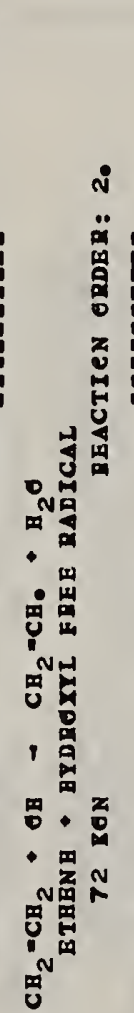 & 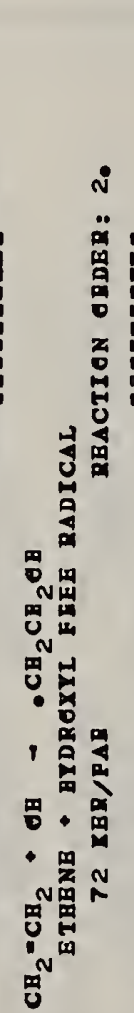 & 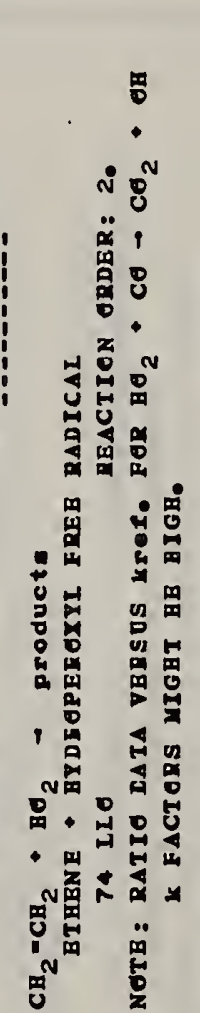 & 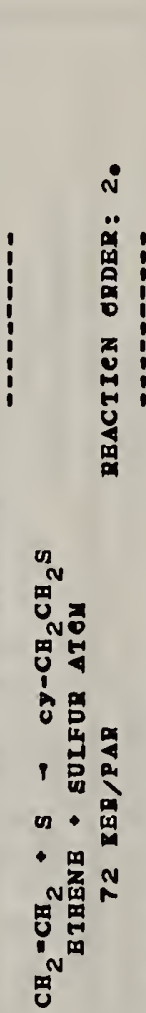 & 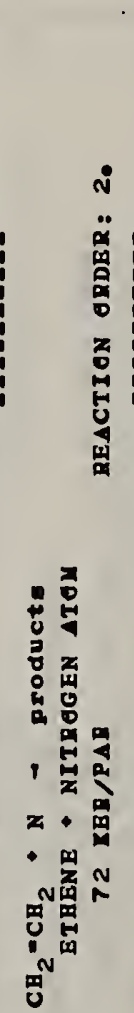 & 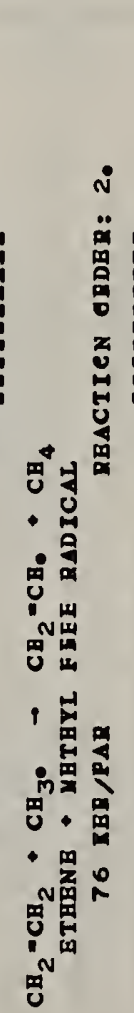 & 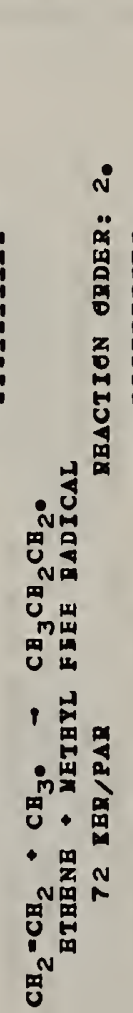 & 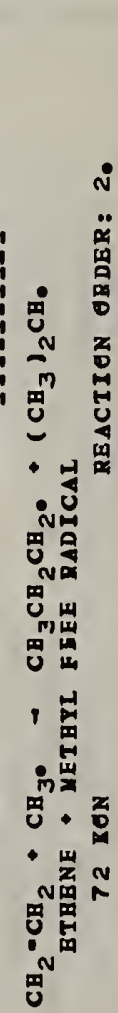 & 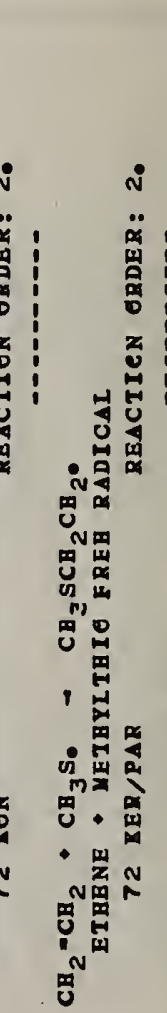 & 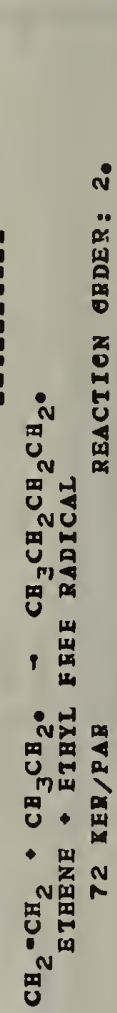 \\
\hline
\end{tabular}




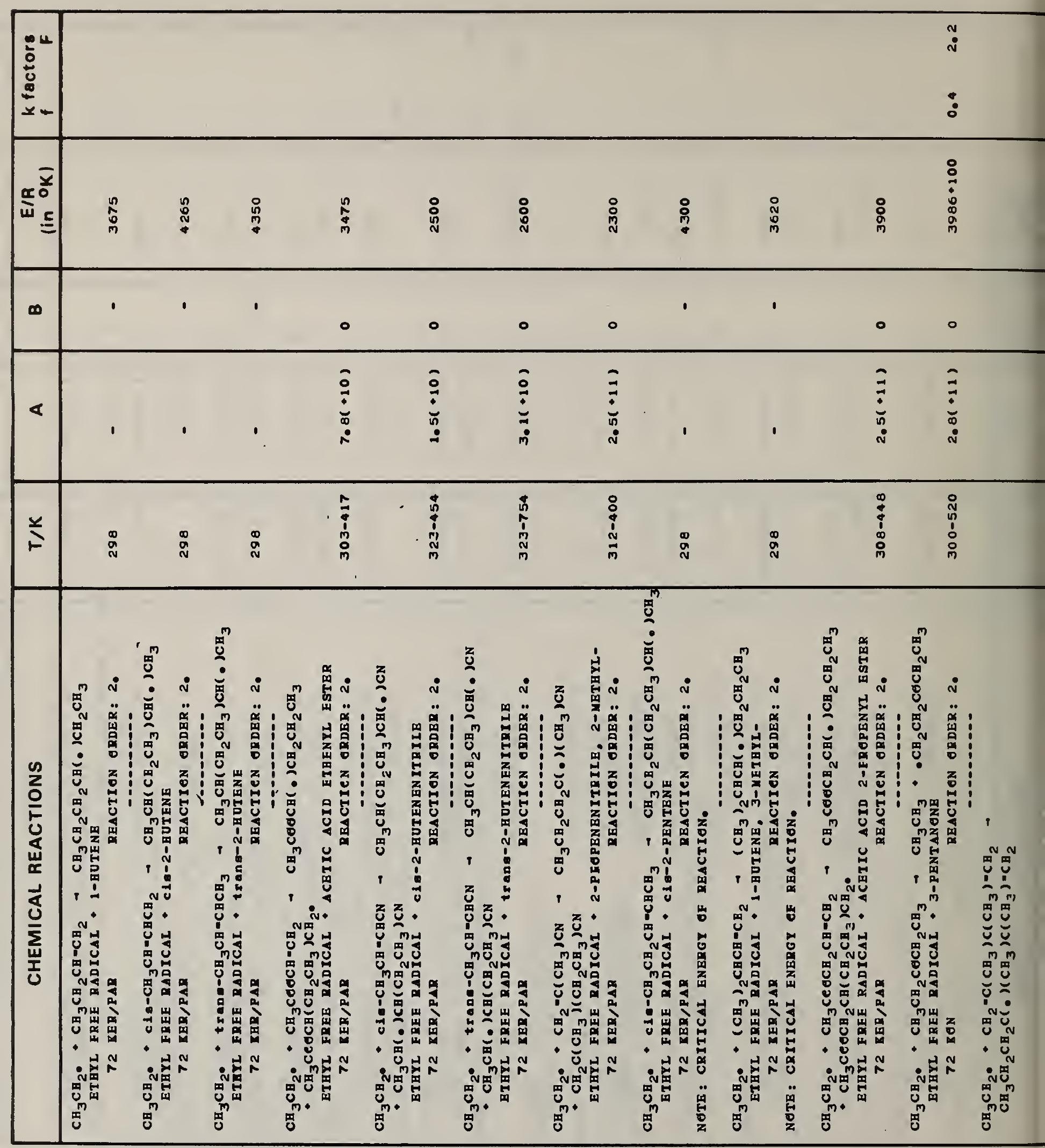




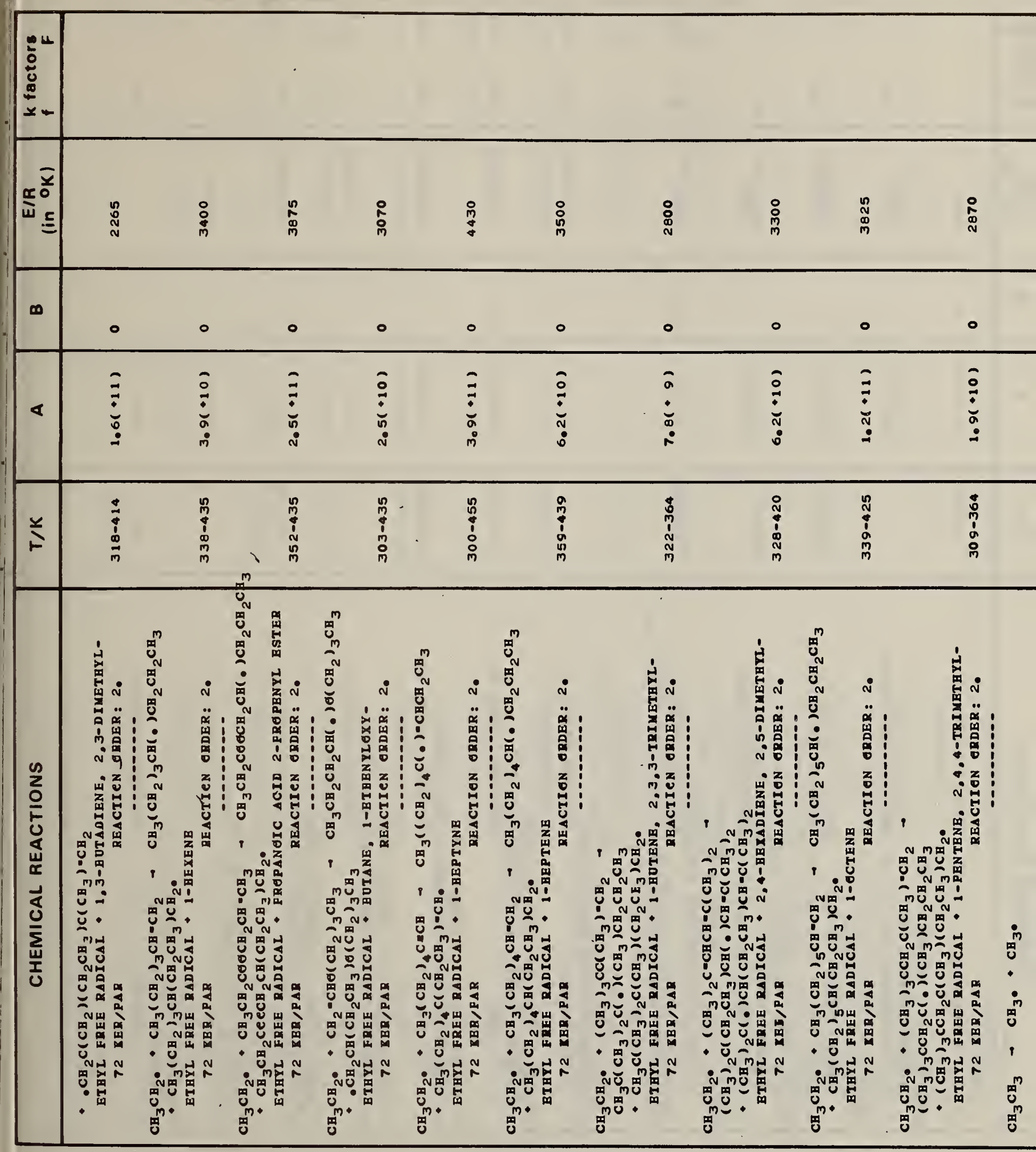




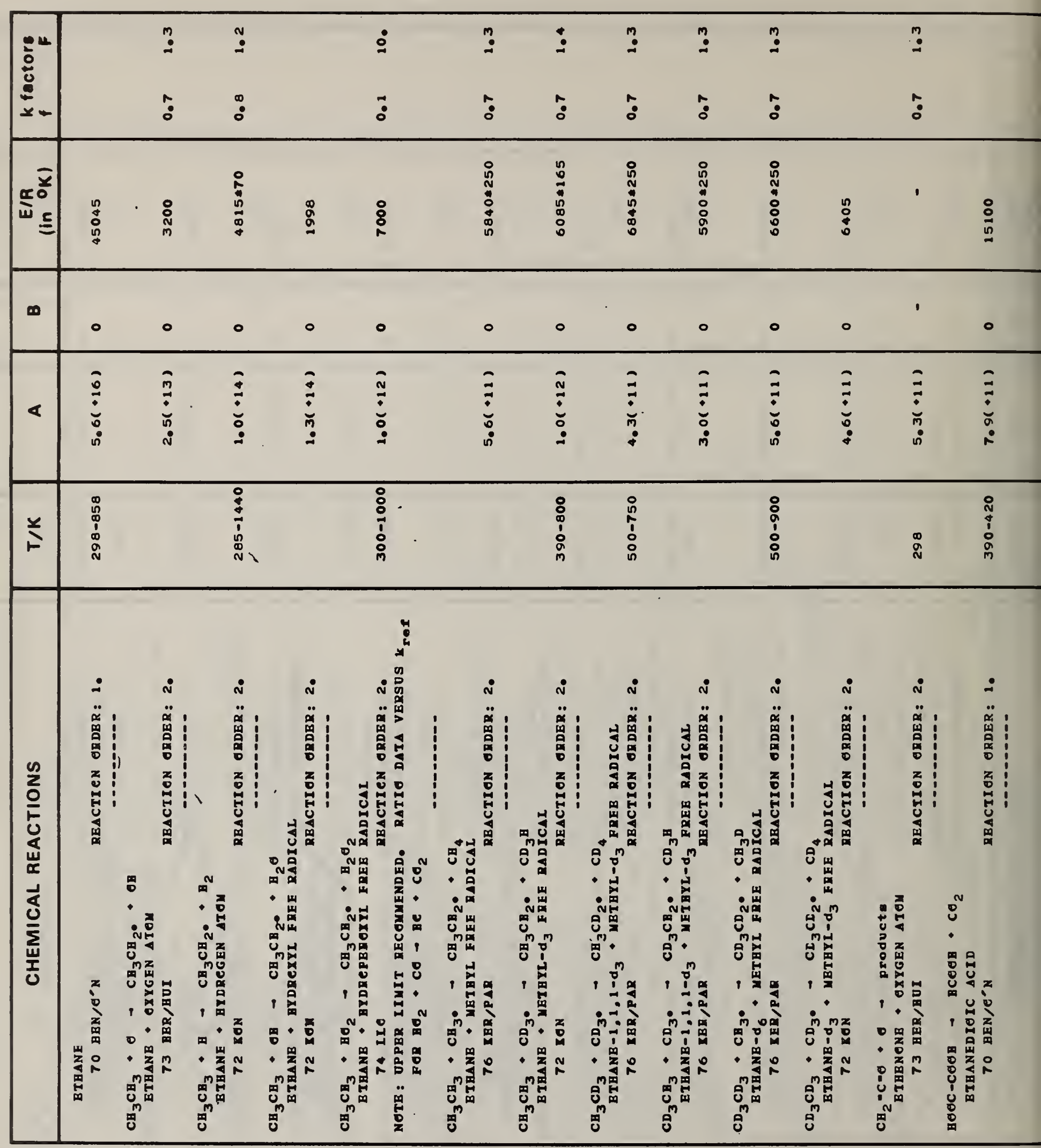




\begin{tabular}{|c|c|c|c|c|c|c|c|c|c|c|c|c|c|}
\hline 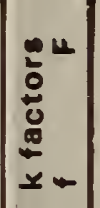 & $\begin{array}{l}: \\
:\end{array}$ & & $\stackrel{\circ}{\dot{N}}$ & $\begin{array}{l}\stackrel{0}{-} \\
\stackrel{\square}{\circ}\end{array}$ & $\begin{array}{l}\stackrel{?}{-} \\
\stackrel{0}{\circ}\end{array}$ & $\begin{array}{l}\stackrel{?}{:} \\
\stackrel{0}{\circ}\end{array}$ & $\begin{array}{l}\text { : } \\
\text { : }\end{array}$ & $\begin{array}{l}n \\
\vdots \\
\vdots \\
:\end{array}$ & \begin{tabular}{l}
$?$ \\
\hdashline \\
$!$
\end{tabular} & $\begin{array}{l}\stackrel{\bullet}{-} \\
\stackrel{0}{\circ}\end{array}$ & $\begin{array}{l}\stackrel{?}{0} \\
\stackrel{0}{\circ}\end{array}$ & $\begin{array}{l}\stackrel{n}{-} \\
\stackrel{0}{0}\end{array}$ & \\
\hline 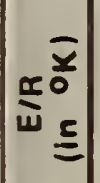 & $\begin{array}{l}n \\
\vdots \\
0 \\
\vdots \\
\hat{0}\end{array}$ & $\begin{array}{l}\text { O. } \\
\text { ng }\end{array}$ & $\stackrel{\wp}{\leftrightarrows}$ & 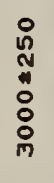 & 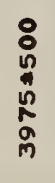 & ' & $\begin{array}{l}0 \\
\stackrel{0}{0} \\
\vdots \\
0 \\
0 \\
\vdots \\
0\end{array}$ & $\begin{array}{l}: \\
: \\
0 \\
: \\
\vdots \\
\vdots\end{array}$ & 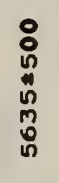 & 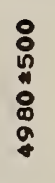 & $\begin{array}{l}: \\
: \\
0 \\
0 \\
\vdots \\
0 \\
0\end{array}$ & 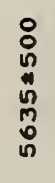 & $\begin{array}{l}\stackrel{R}{a} \\
\tilde{n}\end{array}$ \\
\hline$\infty$ & $\circ$ & $\circ$ & 0 & $\circ$ & 0 & I & ○ & 0 & 0 & - & - & 。 & ○ \\
\hline$\varangle$ & $\begin{array}{l}0 \\
\vdots \\
\vdots\end{array}$ & $\begin{array}{l}\dot{0} \\
\dot{\vdots} \\
\dot{0}\end{array}$ & $\stackrel{m}{:}$ & $\begin{array}{l}0 \\
\vdots \\
\vdots \\
0\end{array}$ & 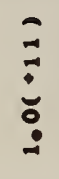 & $\begin{array}{l}\hat{\infty} \\
\dot{\vdots} \\
\dot{0}\end{array}$ & 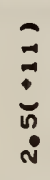 & $\begin{array}{l}\vdots \\
\vdots \\
\dot{\vdots}\end{array}$ & 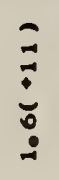 & $\begin{array}{l}\vdots \\
\dot{0} \\
\dot{0}\end{array}$ & $\begin{array}{l}\vdots \\
\dot{n} \\
\dot{n}\end{array}$ & $\bar{\vdots}$ & 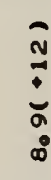 \\
\hline$\stackrel{\text { Y }}{F}$ & 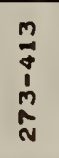 & & 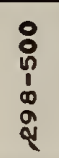 & 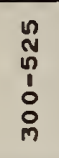 & $\begin{array}{l}: \\
\stackrel{0}{0} \\
\vdots \\
\vdots \\
\dot{n}\end{array}$ & $\stackrel{\infty}{\text { ล̊ }}$ & $\begin{array}{l}8 \\
: \\
1 \\
0 \\
0 \\
p\end{array}$ & $\begin{array}{l}0 \\
\stackrel{h}{0} \\
\vdots \\
0 \\
p\end{array}$ & $\begin{array}{l}\circ \\
\text { h. } \\
\vdots \\
0 \\
n \\
n\end{array}$ & 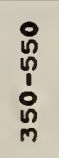 & $\begin{array}{l}\text { o. } \\
\stackrel{h}{0} \\
\vdots \\
0 \\
n\end{array}$ & & $\begin{array}{l}R \\
\hat{i} \\
\mathfrak{R} \\
R\end{array}$ \\
\hline
\end{tabular}

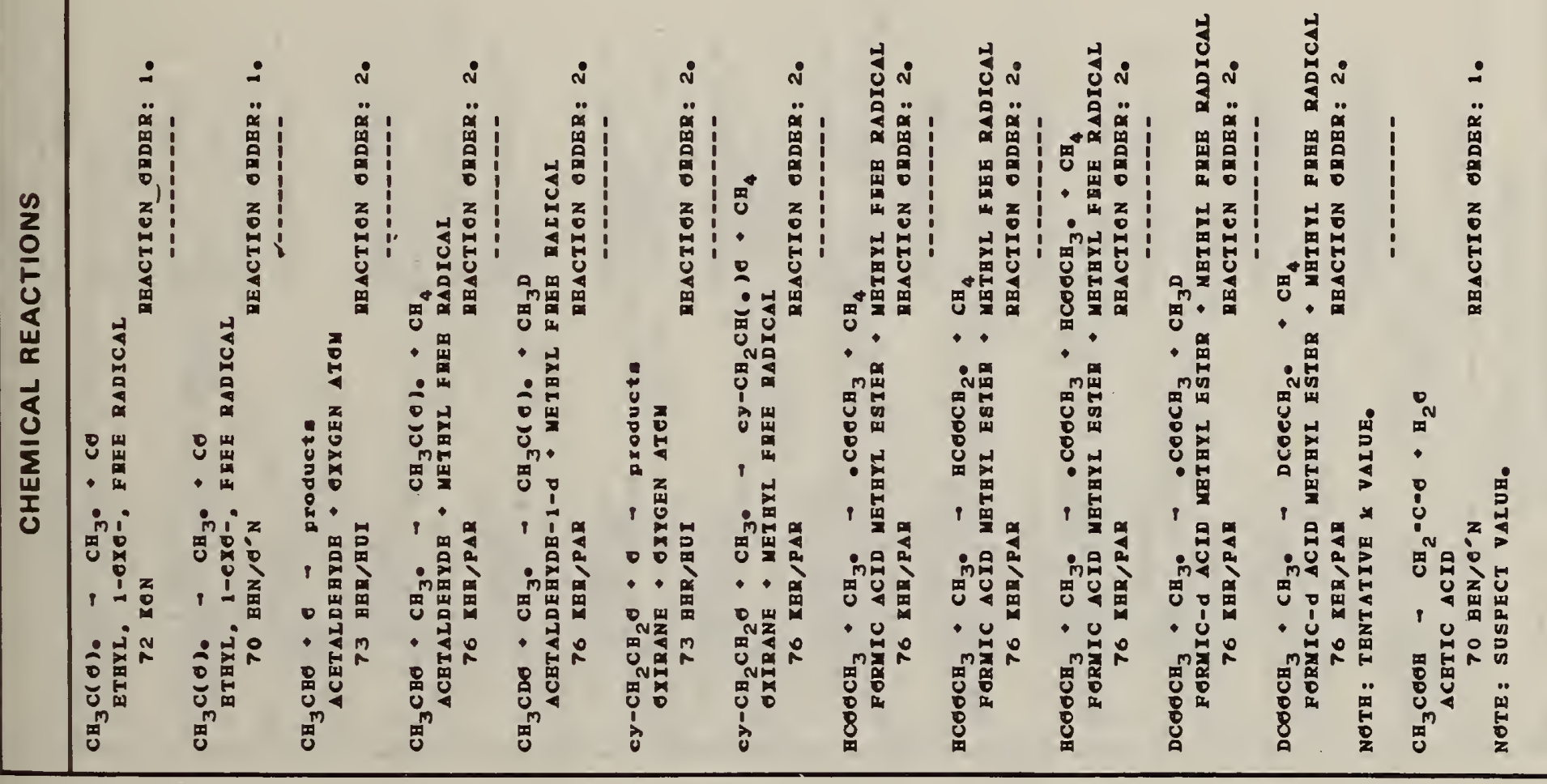




\begin{tabular}{|c|c|c|c|c|c|c|c|c|c|c|c|c|c|}
\hline 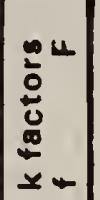 & 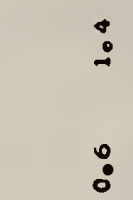 & & $\begin{array}{l}\stackrel{\square}{\circ} \\
\stackrel{0}{\circ}\end{array}$ & $\begin{array}{l}: \\
:\end{array}$ & $\begin{array}{l}\vdots \\
:\end{array}$ & : & $\begin{array}{l}\vdots \\
\vdots\end{array}$ & $\begin{array}{l}\dot{:} \\
:\end{array}$ & $\begin{array}{l}\stackrel{0}{N} \\
\stackrel{0}{:}\end{array}$ & $\begin{array}{l}\vdots \\
\vdots\end{array}$ & & $\begin{array}{l}\text { : } \\
:\end{array}$ & $\begin{array}{l}\stackrel{?}{-} \\
\stackrel{\bullet}{0}\end{array}$ \\
\hline 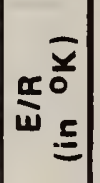 & $\begin{array}{l}: \\
0 \\
0 \\
0 \\
0 \\
m \\
\tilde{n}\end{array}$ & 怘 & ' & $\begin{array}{l}\stackrel{\circ}{0} \\
\stackrel{0}{0} \\
o \\
\stackrel{n}{0} \\
+\end{array}$ & $\begin{array}{l}: \\
: \\
0 \\
: 0 \\
\vdots \\
\vdots \\
0\end{array}$ & $\begin{array}{l}: \\
: \\
: 0 \\
: \\
:\end{array}$ & 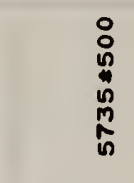 & $\begin{array}{l}: \\
: \\
0 \\
0 \\
0 \\
0 \\
0 \\
8\end{array}$ & 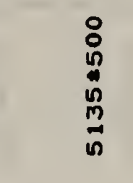 & $\begin{array}{l}: \\
\stackrel{0}{0} \\
\stackrel{0}{0} \\
\vdots \\
\vdots \\
\vdots\end{array}$ & $\begin{array}{l}0 \\
0 \\
\vdots \\
\$\end{array}$ & ָ̊ & $\begin{array}{l}\text { : } \\
: \\
0 \\
0 \\
0 \\
0 \\
0 \\
0\end{array}$ \\
\hline ம & 0 & 0 & ' & ० & 0 & - & $\circ$ & 0 & 0 & 。 & ○ & ० & ० \\
\hline$<$ & $\begin{array}{l}\bar{\vdots} \\
\vdots \\
\vdots\end{array}$ & $\begin{array}{l}\tilde{m} \\
\dot{n} \\
\dot{n}\end{array}$ & مَ & \begin{tabular}{l}
0 \\
\hdashline \\
$\vdots$ \\
$\circ$ \\
$\circ$
\end{tabular} & $\vdots$ & 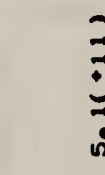 & $\bar{\Xi}$ & & $\underset{\vdots}{\vdots}$ & $\vdots$ & \begin{tabular}{l}
0 \\
$\vdots$ \\
$\vdots$ \\
\hdashline
\end{tabular} & $\begin{array}{l}\hat{N} \\
\vdots \\
\vdots \\
\vdots\end{array}$ & $\bar{\vdots}$ \\
\hline$\stackrel{ }{上}$ & $\begin{array}{l}: \\
\vdots \\
\vdots \\
\vdots \\
0\end{array}$ & 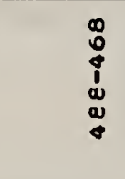 & , & $\begin{array}{l}\text { D̃ } \\
0 \\
\vdots \\
\vdots\end{array}$ & $\begin{array}{l}n \\
0 \\
0 \\
\vdots \\
\vdots \\
\vdots\end{array}$ & 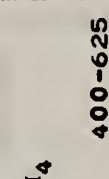 & 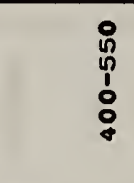 & $\begin{array}{l}0 \\
0 \\
0 \\
0 \\
0 \\
\vdots\end{array}$ & $\begin{array}{l}\text { ñ } \\
0 \\
\vdots \\
\vdots \\
\vdots\end{array}$ & 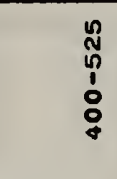 & 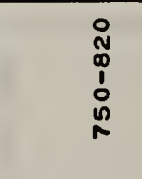 & $\begin{array}{l}0 \\
: \\
0 \\
\vdots \\
\vdots \\
\end{array}$ & $\begin{array}{l}0 \\
\stackrel{0}{0} \\
\stackrel{p}{0} \\
\vdots \\
0\end{array}$ \\
\hline 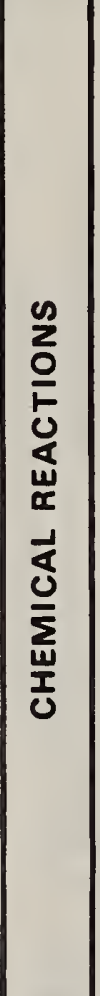 & 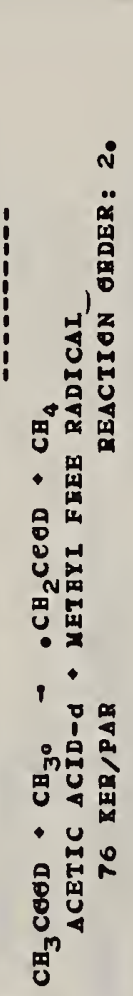 & 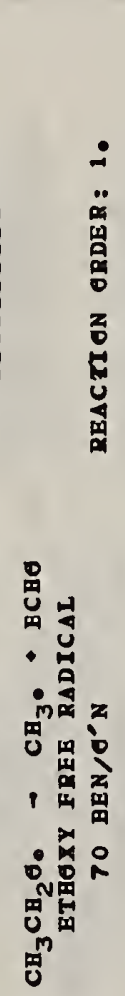 & 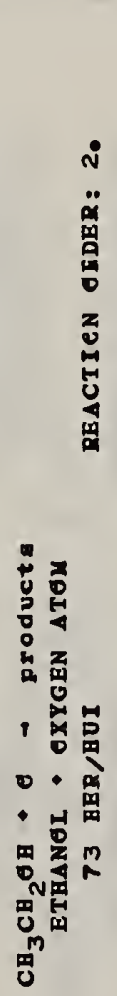 & 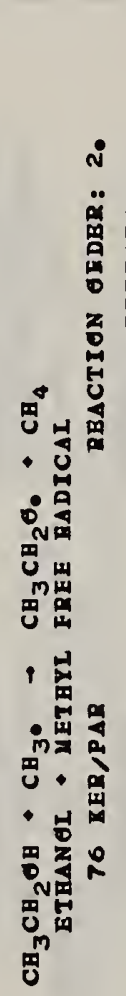 & 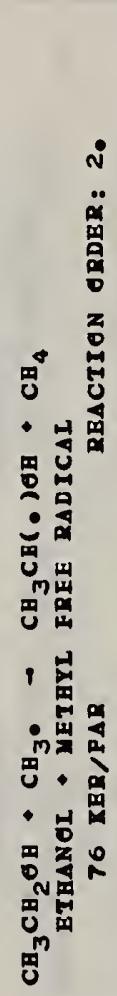 & 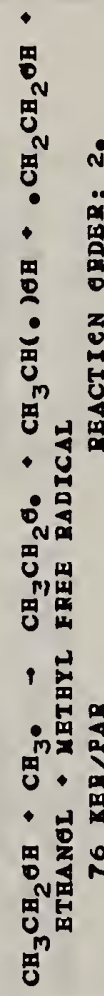 & 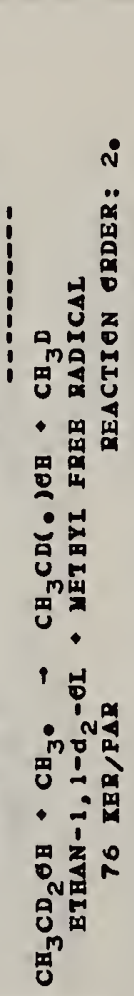 & 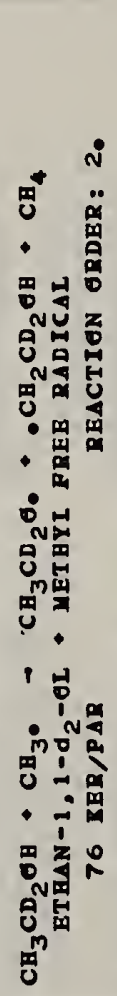 & 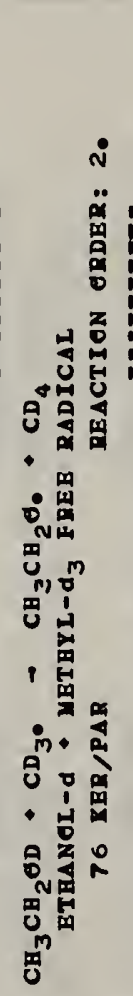 & 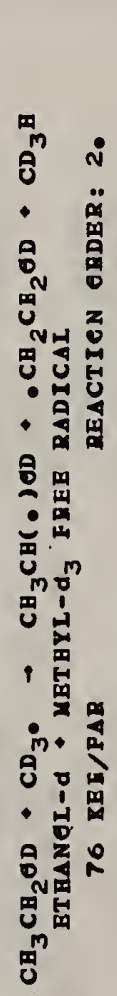 & 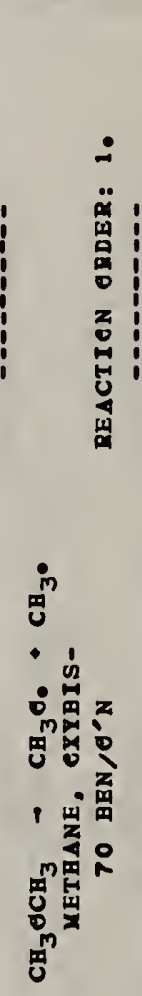 & 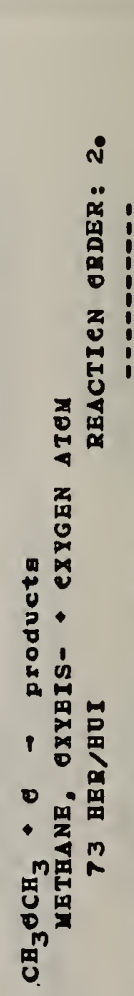 & 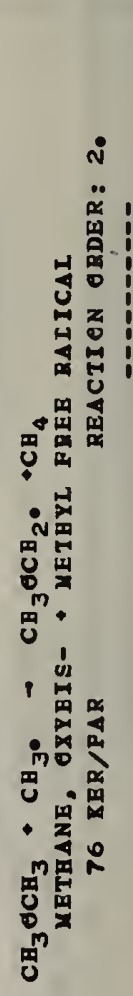 \\
\hline
\end{tabular}




\begin{tabular}{|c|c|c|c|c|c|c|c|c|c|c|c|c|c|c|c|c|c|c|c|}
\hline $\begin{array}{l}04 \\
0 \\
0 \\
0 \\
0 \\
0 \\
x\end{array}$ & & $\begin{array}{l}\dot{0} \\
\dot{0} \\
\dot{m}\end{array}$ & & & & & $\begin{array}{l}: \\
\text { ล } \\
? \\
:\end{array}$ & & & & $\begin{array}{l}: \\
\text { N } \\
\text { ? }\end{array}$ & ?: & & & $\begin{array}{l}: \\
: \\
:\end{array}$ & & $\begin{array}{l}: \\
\text { i } \\
\because\end{array}$ & $\begin{array}{l}: \\
\text { N } \\
\text { : }\end{array}$ & \\
\hline 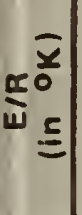 & 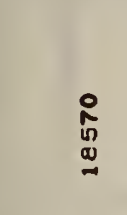 & $\begin{array}{l}: \\
: \\
: 1 \\
: \\
:\end{array}$ & & & $\begin{array}{l}0 \\
\dot{d} \\
0 \\
\text { N }\end{array}$ & & $\begin{array}{l}: \\
0 \\
1 \\
0 \\
0 \\
0 \\
\infty \\
+\end{array}$ & $\begin{array}{l}\text { : } \\
\text { مू } \\
\text { N }\end{array}$ & & 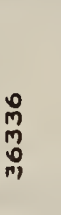 & ' & ' & 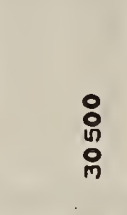 & $\begin{array}{l}\underset{N}{n} \\
\stackrel{N}{0} \\
0\end{array}$ & $\begin{array}{l}: \\
8 \\
17 \\
: 8 \\
: \\
\text { in }\end{array}$ & & 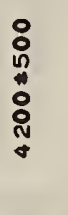 & $\begin{array}{l}: \\
0 \\
0 \\
: \\
0 \\
0 \\
0\end{array}$ & \\
\hline$\infty$ & 0 & 0 & & & 0 & & $\circ$ & ० & & 0 & ' & I & 0 & 0 & 0 & & 0 & - & \\
\hline$\varangle$ & $\begin{array}{l}5 \\
\vdots \\
\dot{0}\end{array}$ & 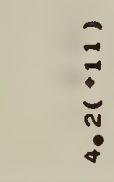 & & & 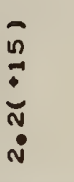 & & 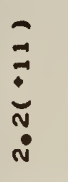 & $\begin{array}{l}\tilde{m} \\
\vdots \\
\vdots\end{array}$ & & $\frac{\tilde{n}}{\vdots}$ & $\stackrel{0}{\dot{n}}$ & 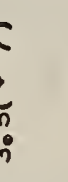 & $\begin{array}{l}\vdots \\
\vdots \\
\vdots \\
\vdots\end{array}$ & 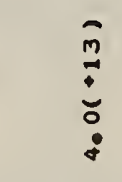 & $\begin{array}{l}\bar{\vdots} \\
\dot{5}\end{array}$ & & $\begin{array}{l}\Xi \\
\vdots \\
\dot{N}\end{array}$ & $\begin{array}{l}\vdots \\
\vdots \\
\vdots\end{array}$ & 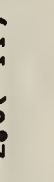 \\
\hline$\stackrel{y}{\vdash}$ & $\begin{array}{l}N \\
n \\
0 \\
1 \\
1 \\
n \\
0 \\
m\end{array}$ & $\begin{array}{l}: \\
: \\
i \\
\vdots \\
\text { in } \\
m\end{array}$ & & 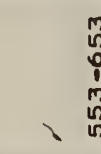 & 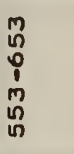 & & $\begin{array}{l}: \\
: \\
1 \\
\vdots \\
:\end{array}$ & $\begin{array}{l}\infty \\
0 \\
0 \\
1 \\
1 \\
w \\
w \\
N\end{array}$ & & 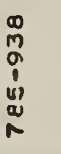 & 吕 & ? & $\begin{array}{l}m \\
a \\
i \\
m \\
\infty \\
n\end{array}$ & 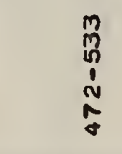 & $\begin{array}{l}: \\
: \\
0 \\
\vdots \\
0 \\
n\end{array}$ & & $\begin{array}{l}: \\
: \\
1 \\
\vdots \\
1 \\
n\end{array}$ & 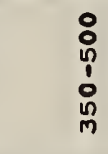 & \\
\hline 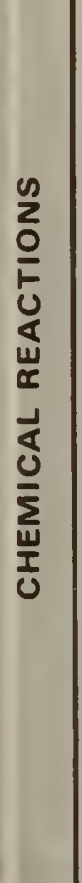 & $\begin{array}{l} \\
\end{array}$ & 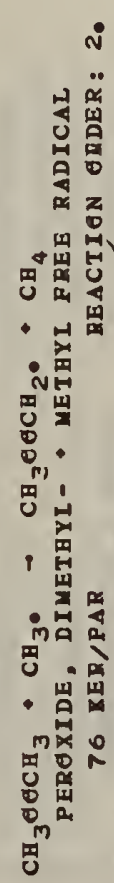 & 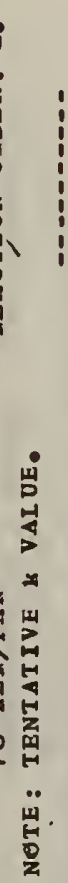 & 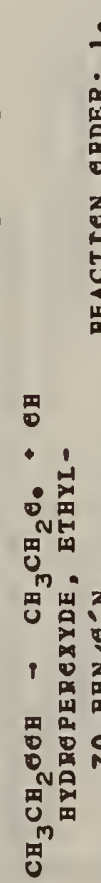 & 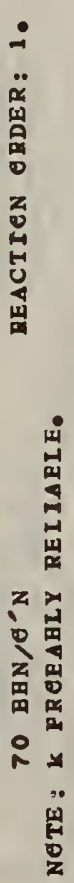 & 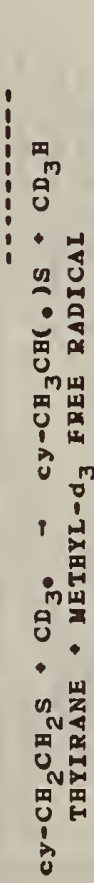 & 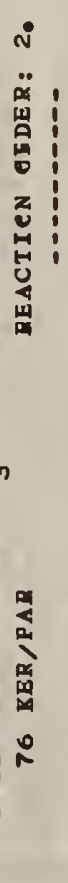 & 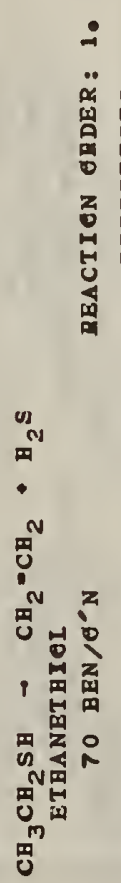 & 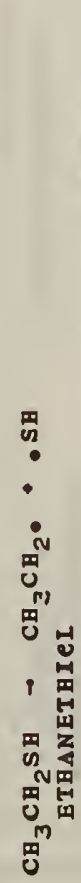 & 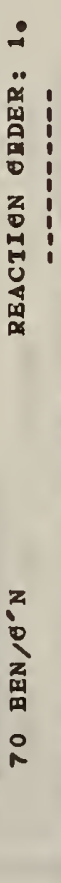 & 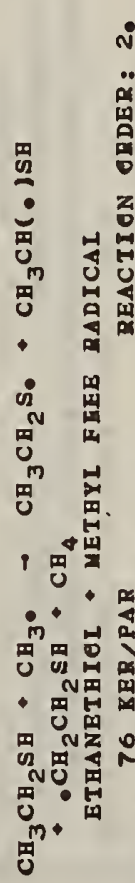 & 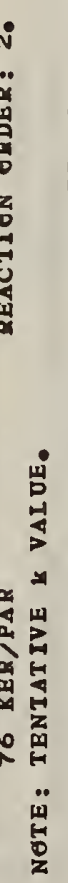 & $\begin{array}{l}\text { a } \\
\\
\end{array}$ & 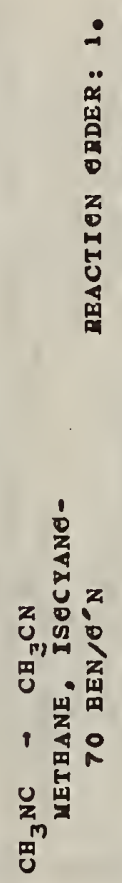 & 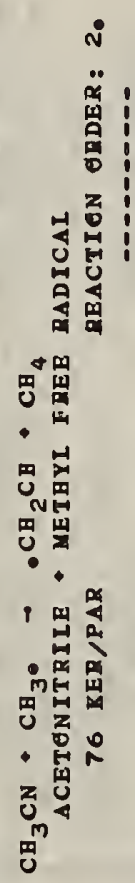 & 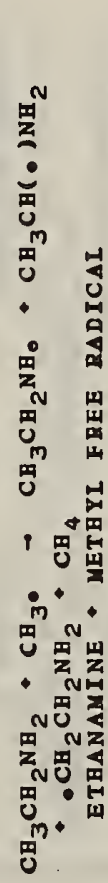 & 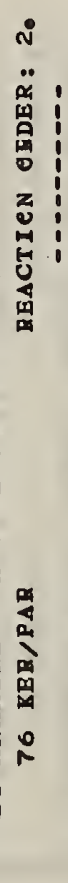 & 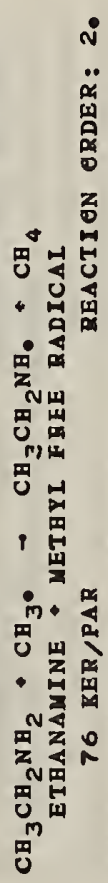 & : \\
\hline
\end{tabular}




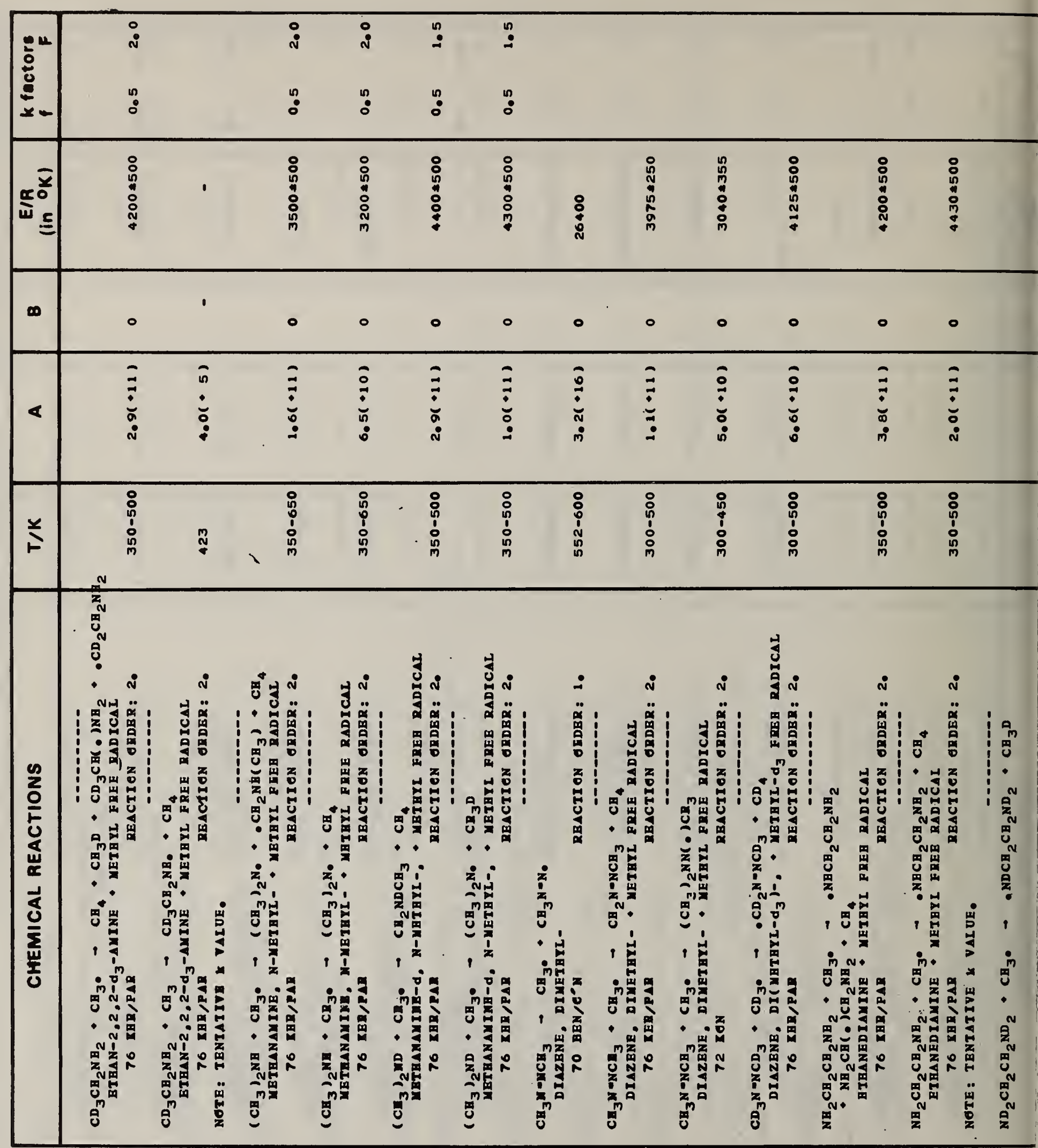




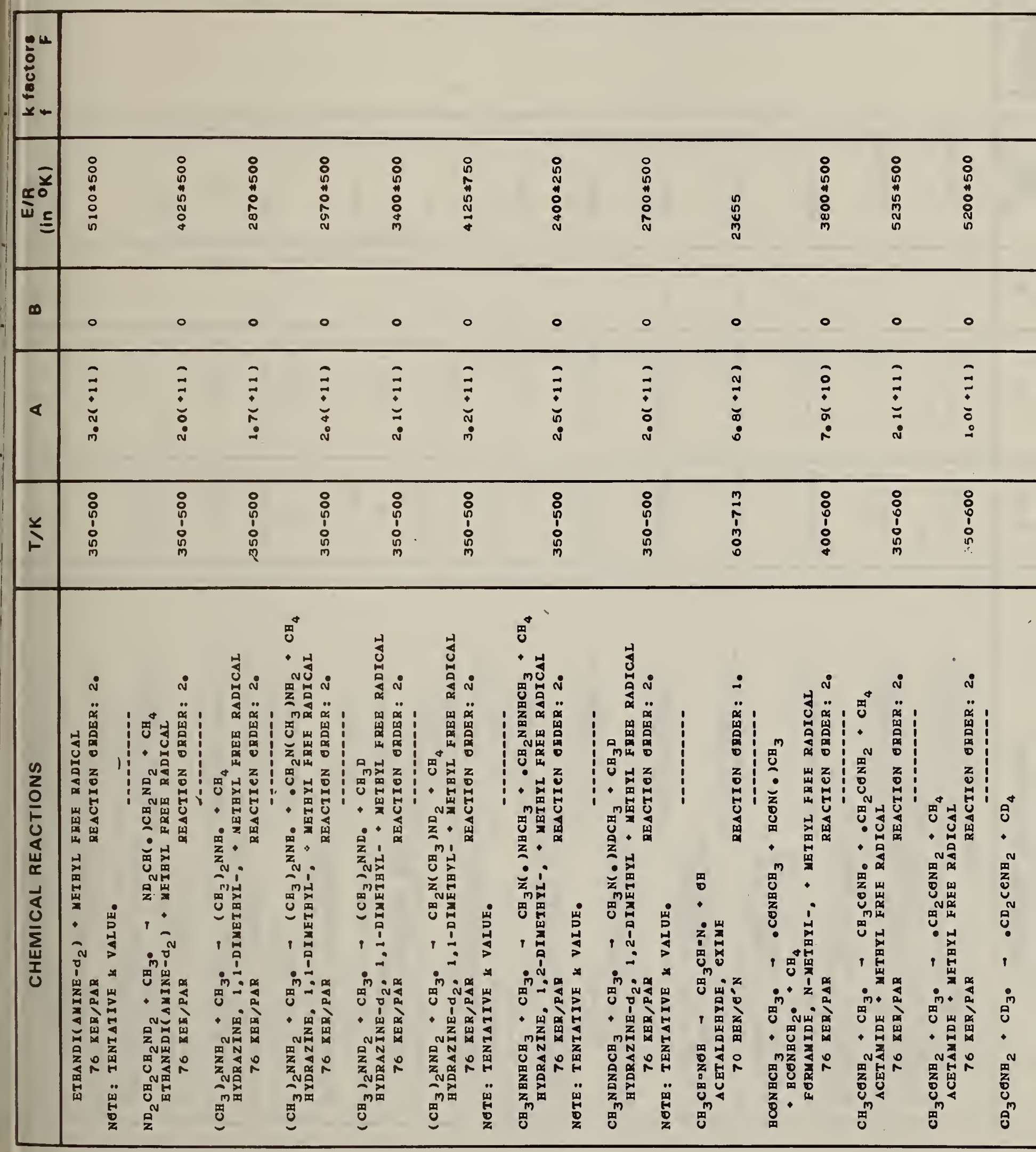




\begin{tabular}{|c|c|c|c|c|c|c|c|c|c|c|c|c|c|}
\hline 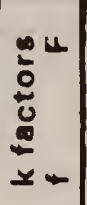 & & & & & & & & $\begin{array}{l}: \\
\text { N } \\
?\end{array}$ & & & & & • \\
\hline 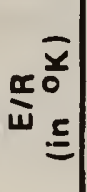 & 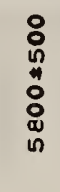 & 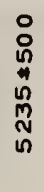 & 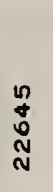 & $\begin{array}{l}\text { : } \\
\dot{\$} \\
\text { ㅇ }\end{array}$ & $\begin{array}{l}\stackrel{0}{M} \\
0 \\
a \\
a\end{array}$ & $\begin{array}{l}0 \\
\not \\
0 \\
0 \\
0\end{array}$ & $\begin{array}{l}: \\
: \\
\stackrel{-}{=}\end{array}$ & ' & 1 & 1 & $\stackrel{n}{R}$ & I & $\stackrel{8}{\circ}$ \\
\hline $\boldsymbol{\infty}$ & 0 & 0 & 0 & 0 & 。 & 0 & 0 & I. & I & 1 & 0 & I & 0 \\
\hline$\alpha$ & $\begin{array}{l} \pm \\
\Xi \\
\vdots\end{array}$ & $\begin{array}{l}\Xi \\
\vdots\end{array}$ & $\begin{array}{l}\vec{N} \\
\stackrel{0}{0} \\
\text { n }\end{array}$ & $\begin{array}{l}\overrightarrow{0} \\
\dot{0} \\
\dot{0}\end{array}$ & $\begin{array}{l}\hat{0} \\
\dot{n} \\
\dot{n}\end{array}$ & $\begin{array}{l}0 \\
\vdots \\
\vdots \\
\circ\end{array}$ & $\begin{array}{l}\tilde{m} \\
\vdots \\
\vdots \\
\text { i }\end{array}$ & $\begin{array}{l} \pm \\
\vdots \\
\vdots\end{array}$ & $\begin{array}{l}\Xi \\
\vdots \\
\vdots\end{array}$ & $\underset{\mathfrak{N}}{\stackrel{0}{\vdots}}$ & $\begin{array}{l}0 \\
\vdots \\
\vdots \\
0\end{array}$ & 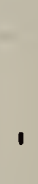 & $\begin{array}{l}\vec{y} \\
\dot{0} \\
0 \\
\text { in }\end{array}$ \\
\hline ㄴ & $\begin{array}{l}: \\
: \\
0 \\
1 \\
0 \\
n \\
m\end{array}$ & $\begin{array}{l}8 \\
8 \\
0 \\
1 \\
0 \\
10 \\
m\end{array}$ & 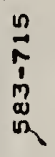 & $\begin{array}{l}n \\
0 \\
0 \\
1 \\
\vdots \\
0 \\
+\end{array}$ & $\begin{array}{l}\$ \\
\$ \\
\vdots \\
\vdots \\
\vdots\end{array}$ & $\begin{array}{l}\infty \\
\stackrel{\infty}{n} \\
n \\
\infty \\
\infty \\
n \\
m\end{array}$ & $\begin{array}{l}\vdots \\
\vdots \\
\vdots \\
\vdots \\
\vdots \\
m\end{array}$ & $\begin{array}{l}\boldsymbol{\omega} \\
\stackrel{\sigma}{N}\end{array}$ & $\stackrel{\infty}{\stackrel{\infty}{\sim}}$ & $\begin{array}{c}\infty \\
\stackrel{\alpha}{N}\end{array}$ & $\begin{array}{l}\text { O } \\
\text { N } \\
1 \\
\vdots \\
N \\
m\end{array}$ & 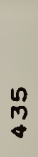 & $\begin{array}{l}n \\
0 \\
\vdots \\
\vdots \\
\alpha \\
n \\
n\end{array}$ \\
\hline
\end{tabular}

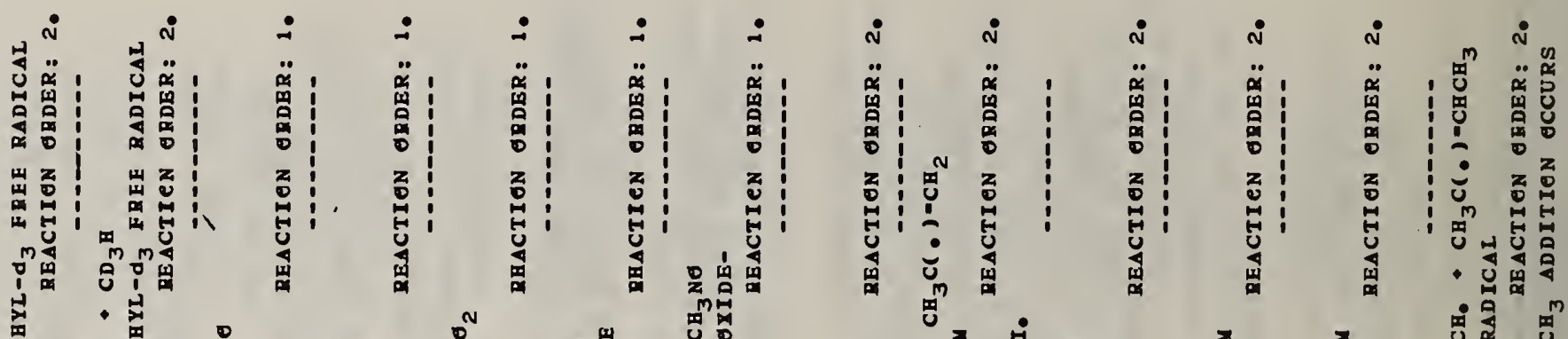

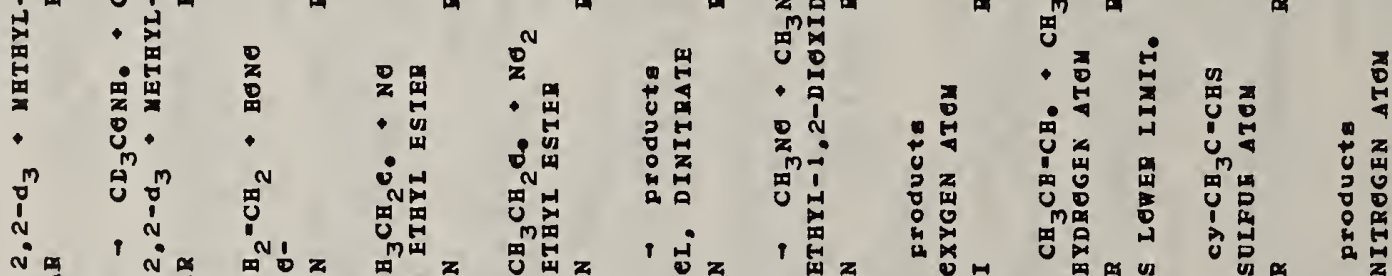

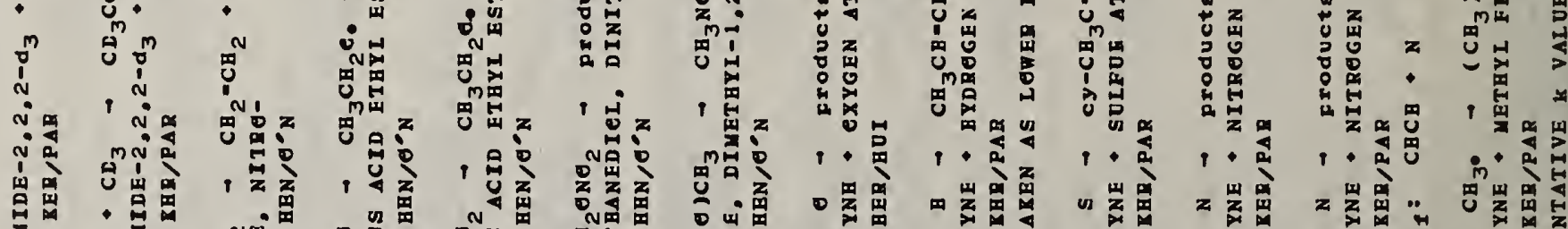

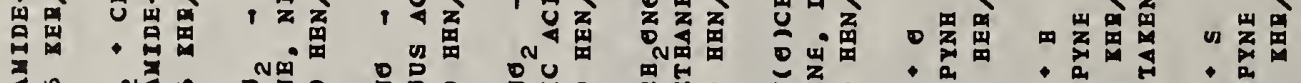

出

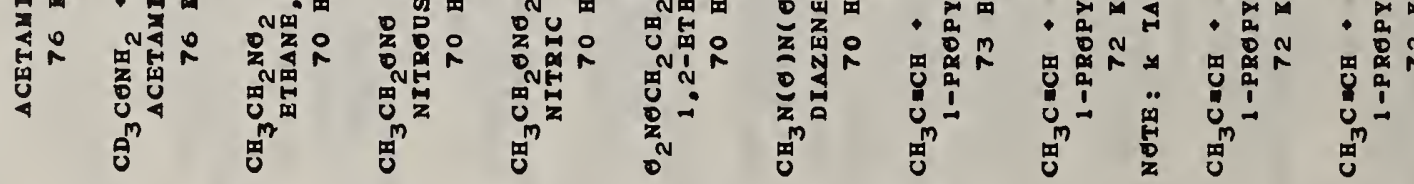

壁

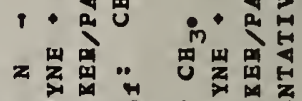

-

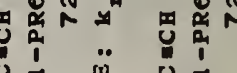




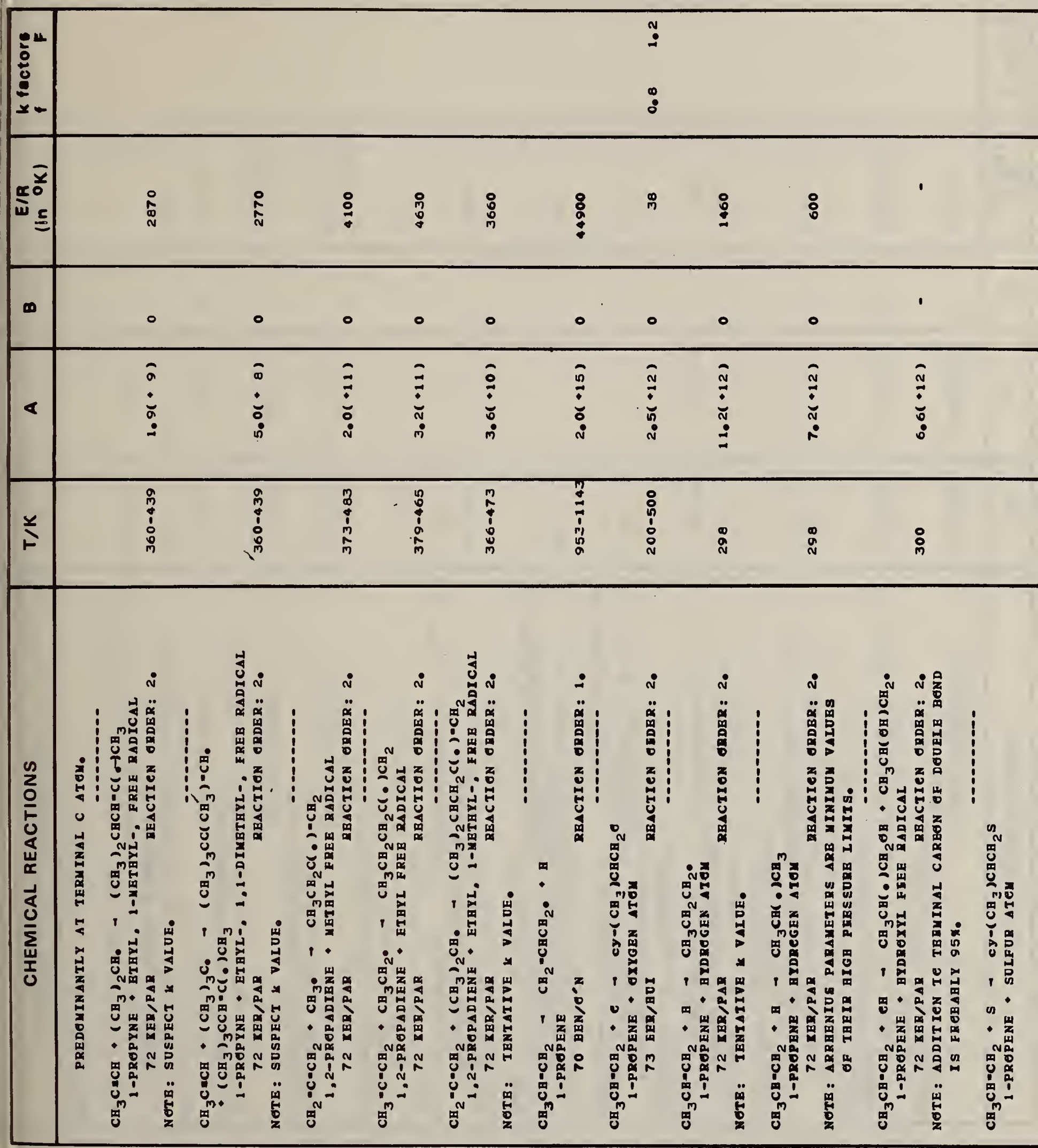




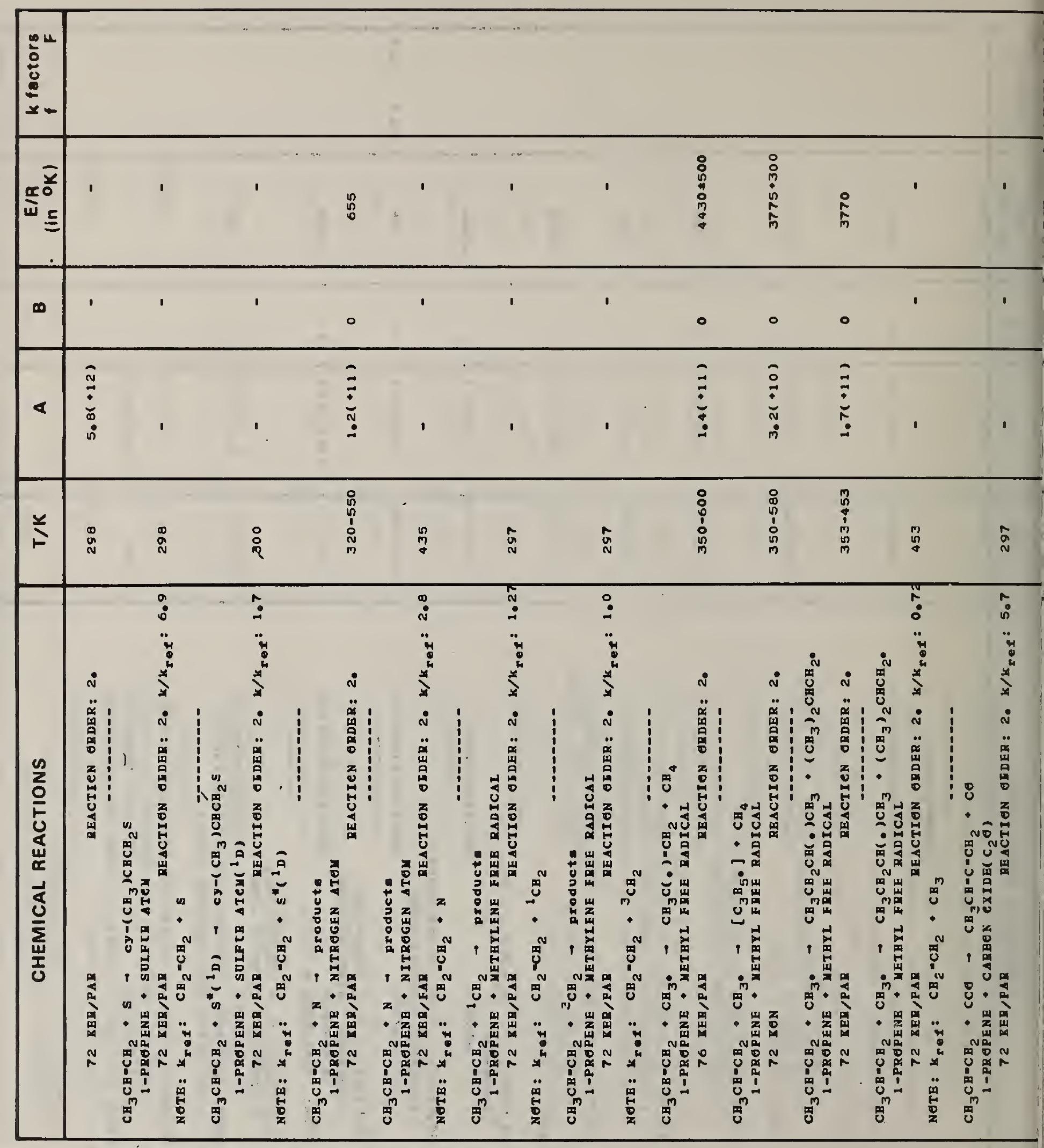




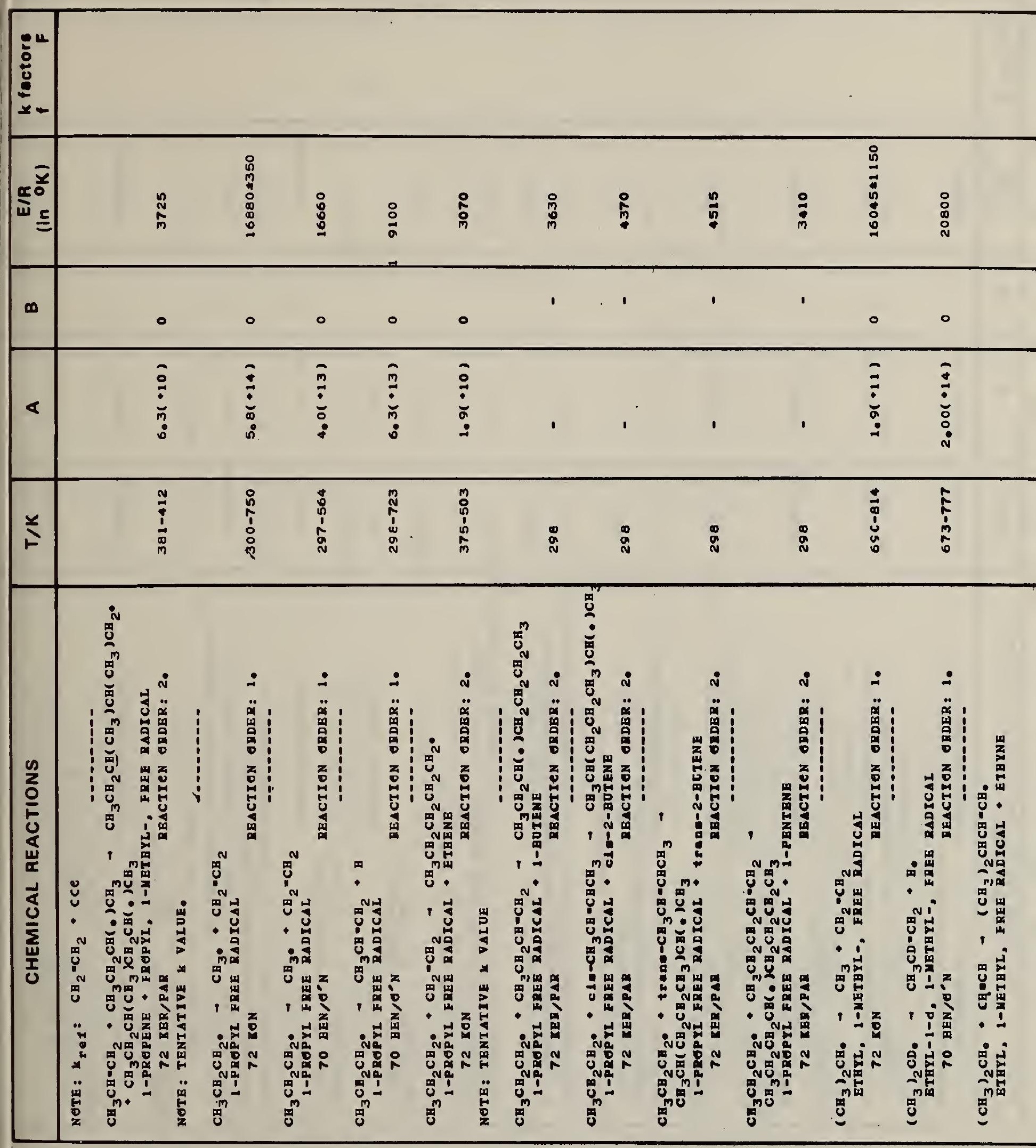




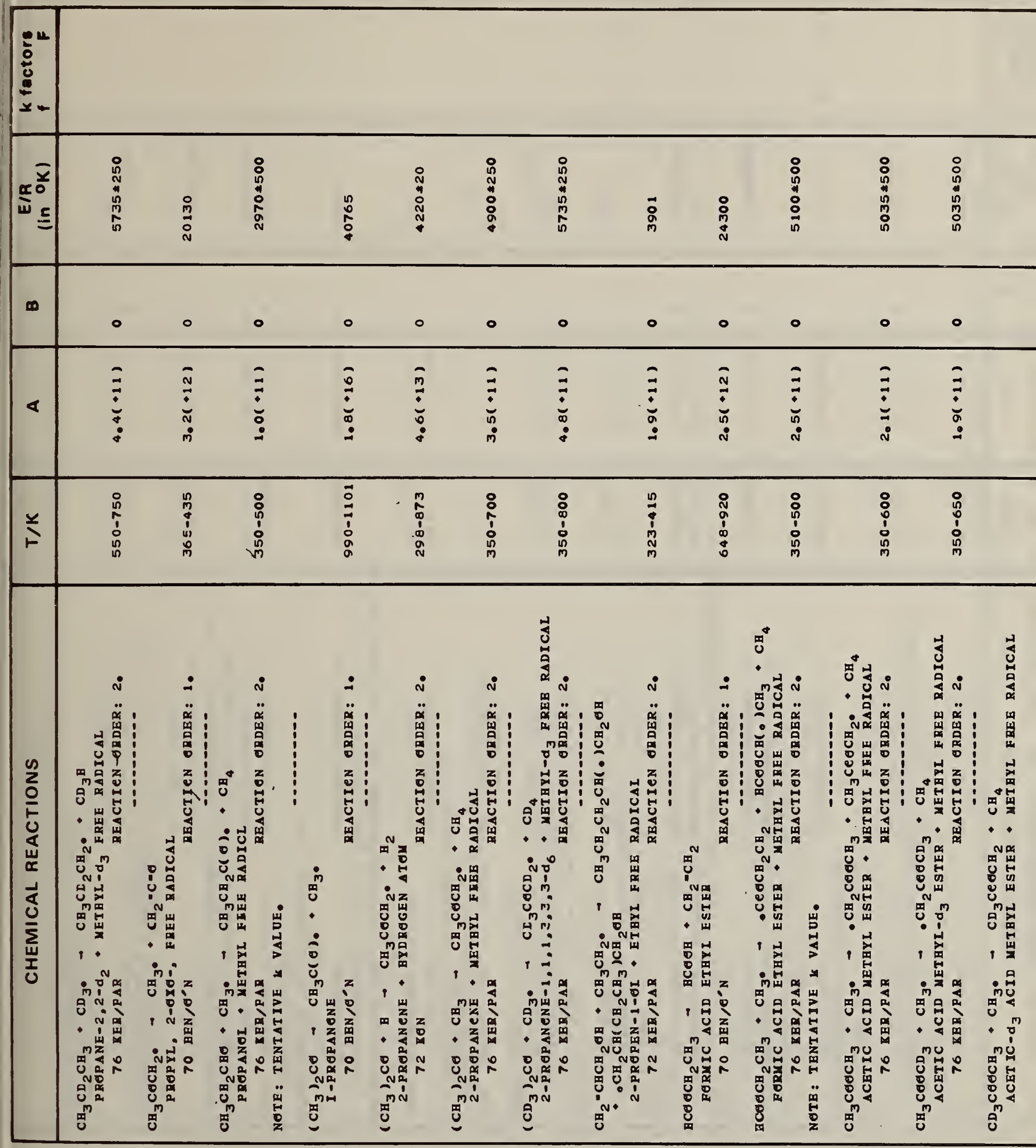




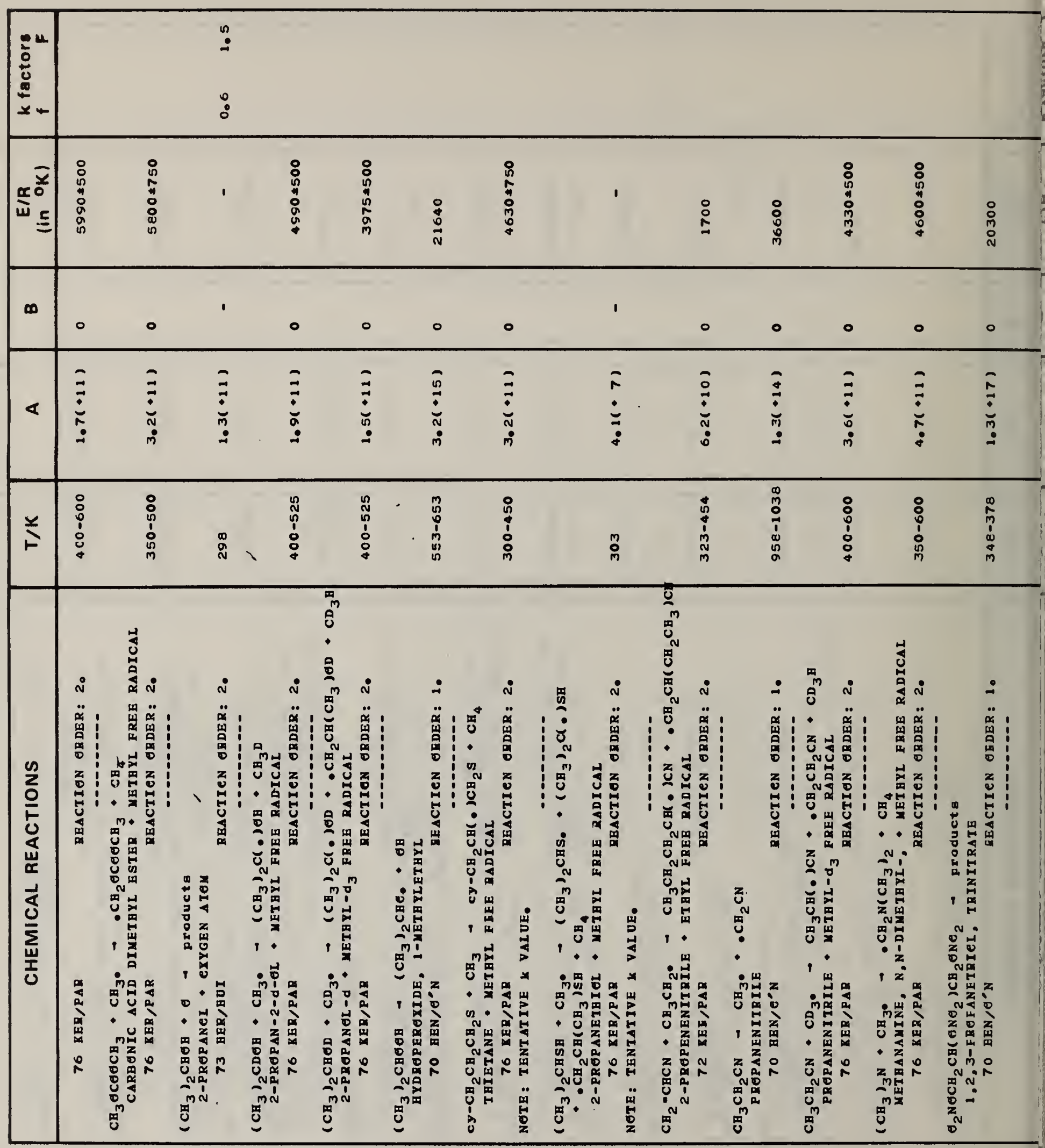




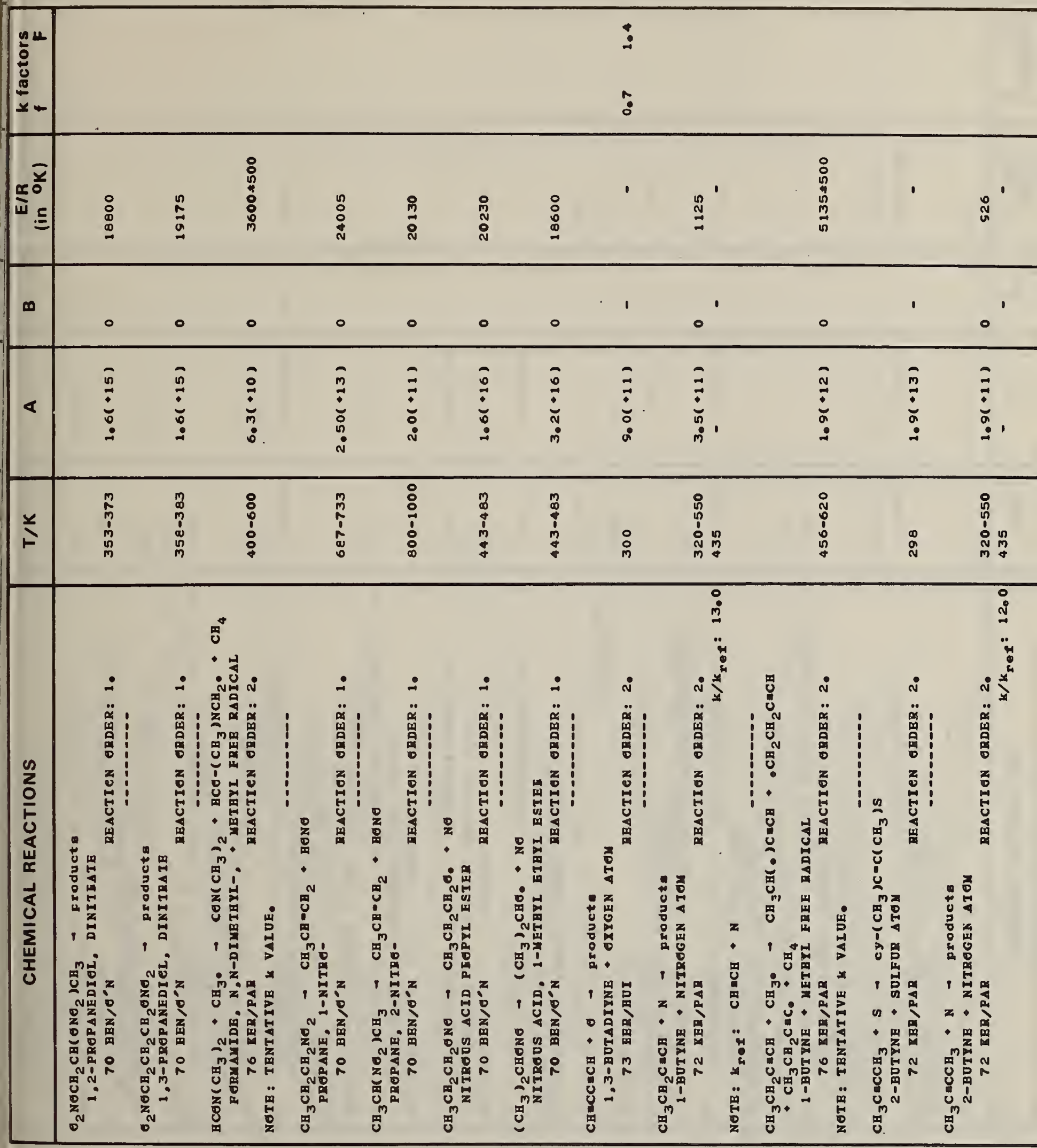




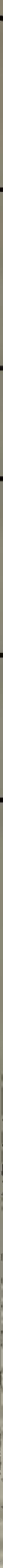




\begin{tabular}{|c|c|c|c|c|c|c|c|c|c|c|c|c|c|c|c|c|c|c|c|}
\hline 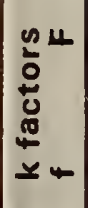 & $\begin{array}{l}: \\
: \\
\$ \\
:\end{array}$ & & & & & & & & & & & & & & & & & $=$ & \\
\hline 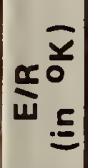 & I & 1 & & & ' & & 1 & & 1 & : & & ' & & ' & & & 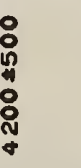 & ${ }^{\prime}$ & \\
\hline m & . & ' & & & ' & & . & & . ' & & 1 & ' & & - & & & - & $0^{\prime}$ & \\
\hline$\varangle$ & $\begin{array}{l}\hat{N} \\
\dot{m} \\
\text { s. }\end{array}$ & $\vec{\Xi}$ & & & \begin{tabular}{l}
0 \\
\hdashline \\
$\vdots$ \\
$\circ$
\end{tabular} & & $\begin{array}{l}\equiv \\
\equiv\end{array}$ & & 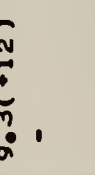 & $\begin{array}{l}= \\
\vdots \\
0\end{array}$ & & ' & & ' & & & $\begin{array}{l}5 \\
\vdots \\
5 \\
0\end{array}$ & $\begin{array}{l}\vdots \\
\vdots \\
\vdots\end{array}$ & \\
\hline$\stackrel{Y}{F}$ & $\stackrel{\infty}{\overbrace{N}^{\infty}}$ & 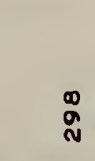 & & & $\stackrel{\infty}{\stackrel{\infty}{N}}$ & & $\begin{array}{l}\infty \\
\stackrel{\infty}{N}\end{array}$ & & $\begin{array}{c}\infty \\
\infty \\
N\end{array}$ & $\begin{array}{l}\circ \\
\stackrel{n}{0} \\
0 \\
\vdots \\
\stackrel{N}{0} \\
m\end{array}$ & & å & & à & & & $\begin{array}{l}8 \\
8 \\
0 \\
0 \\
8 \\
8 \\
0\end{array}$ & 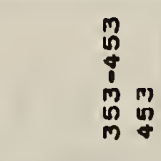 & \\
\hline 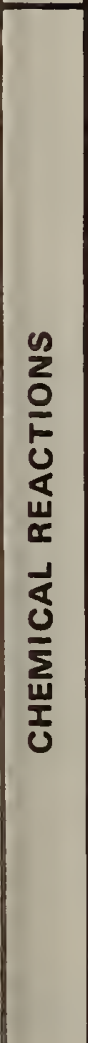 & 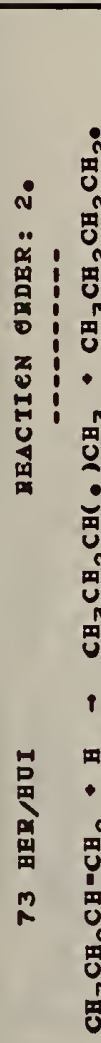 & 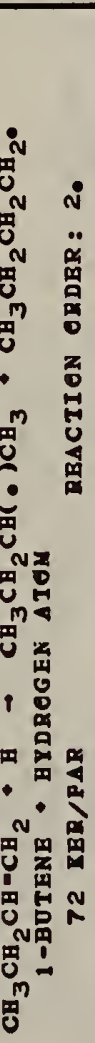 & 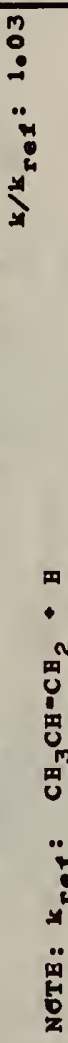 & 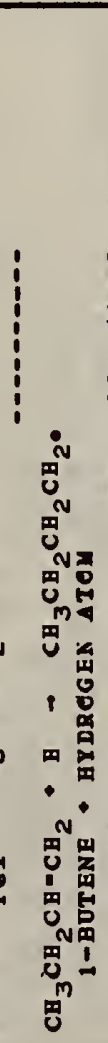 & 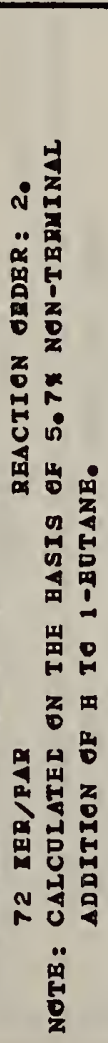 & 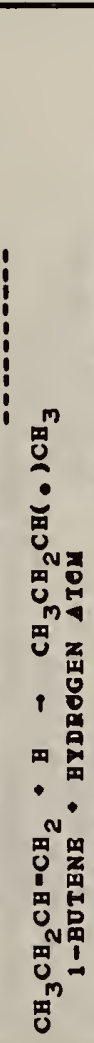 & 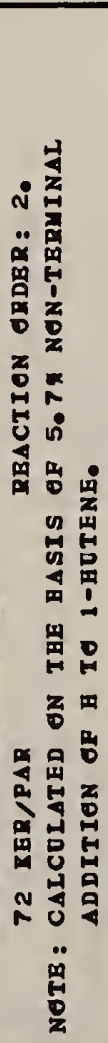 & 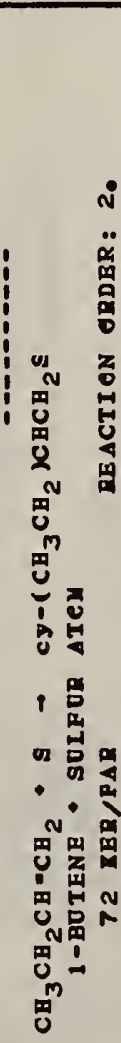 & : & 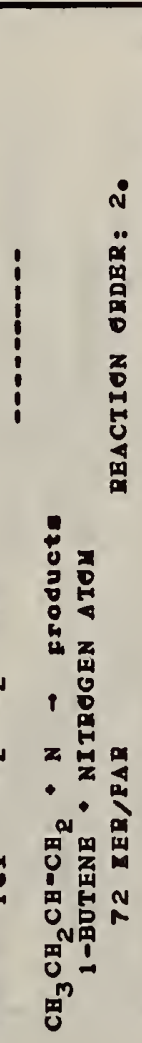 & 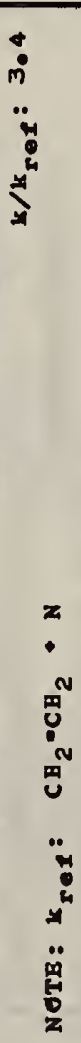 & $\begin{array}{l} \\
\end{array}$ & 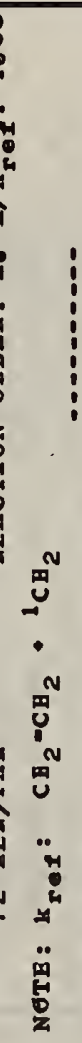 & 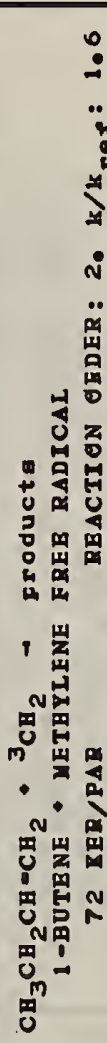 & 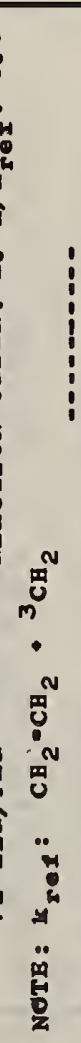 & 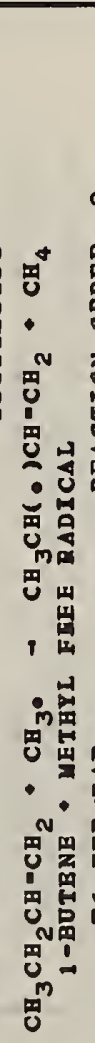 & 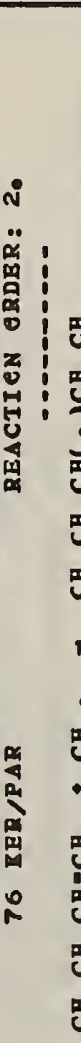 & 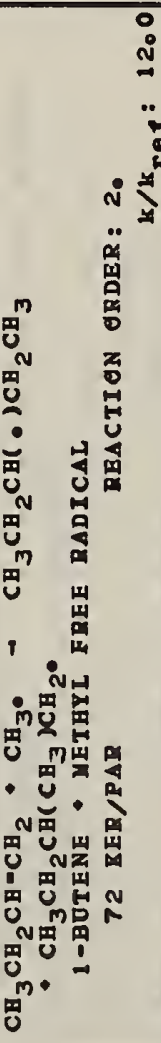 & 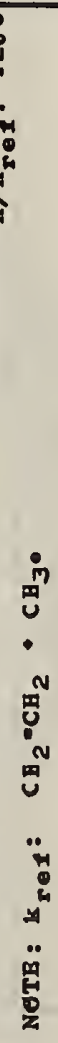 \\
\hline
\end{tabular}




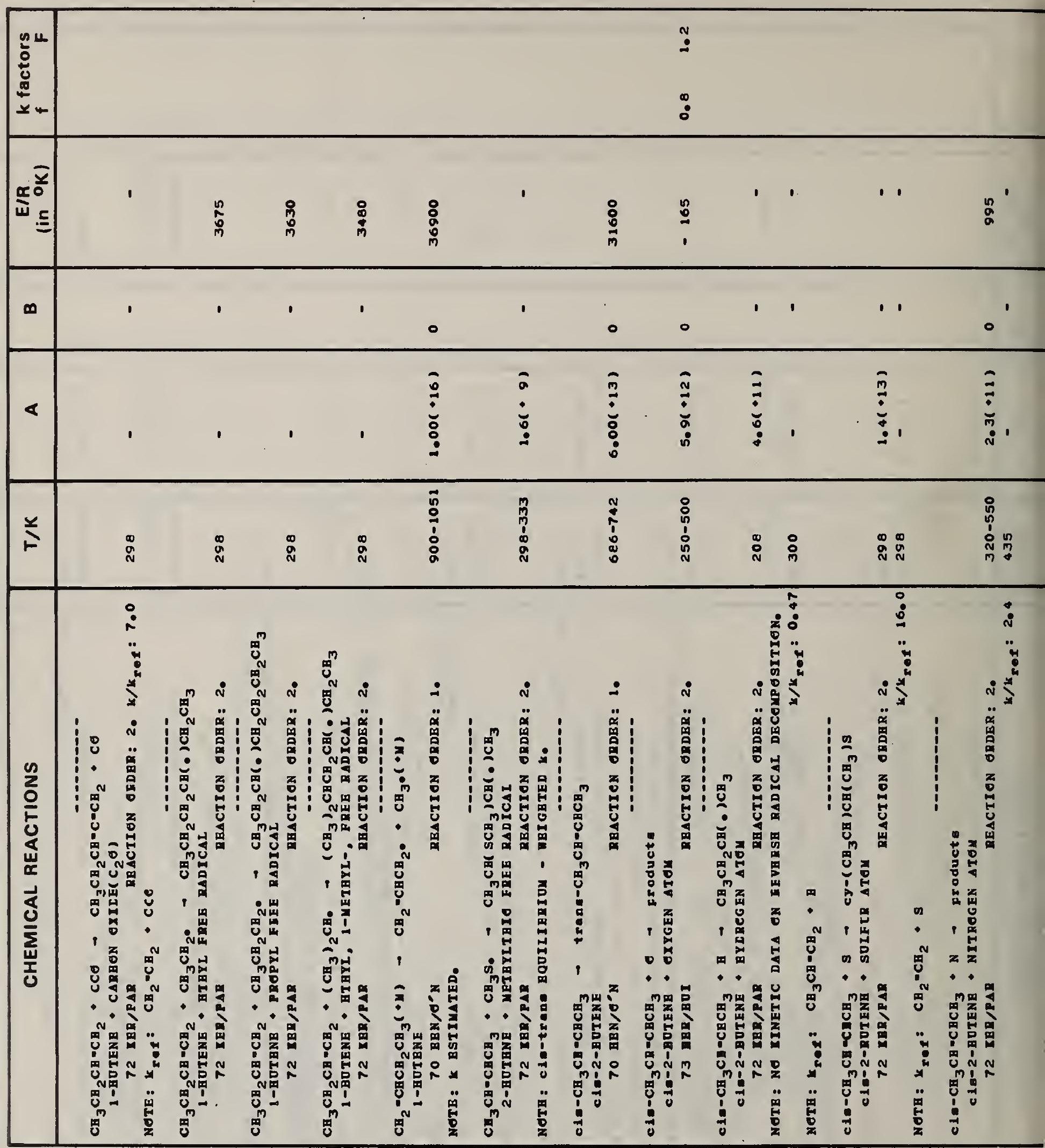




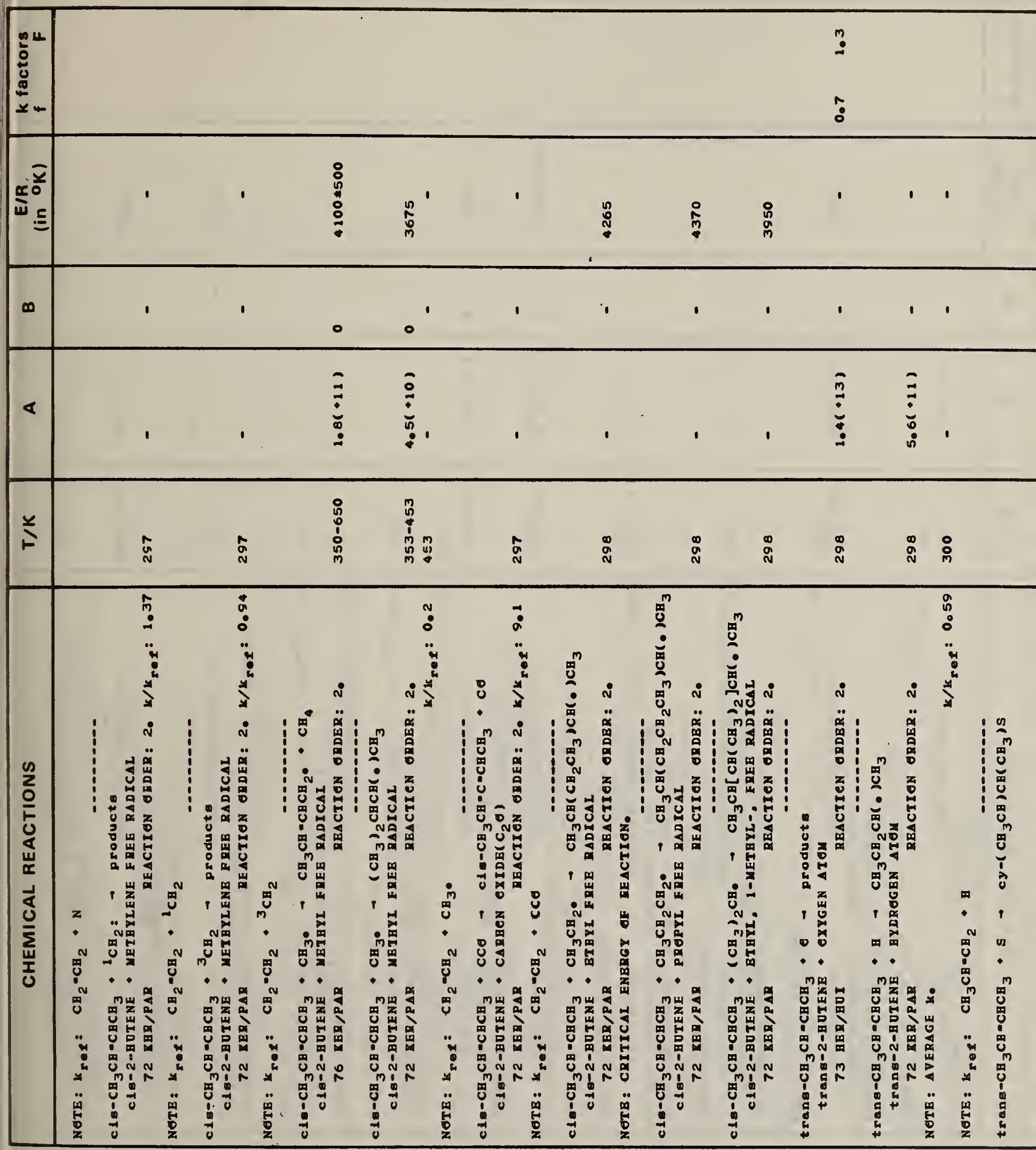




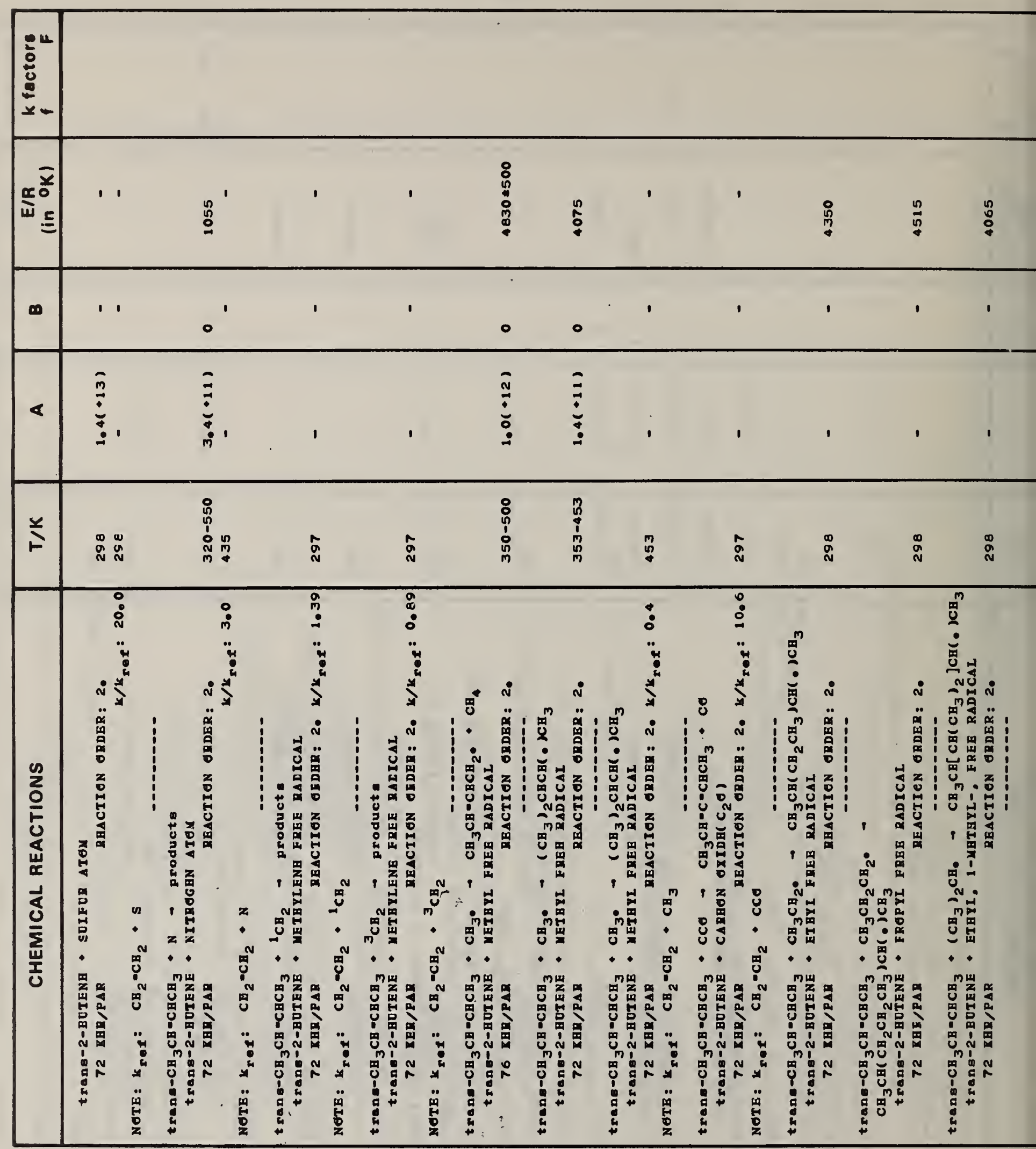




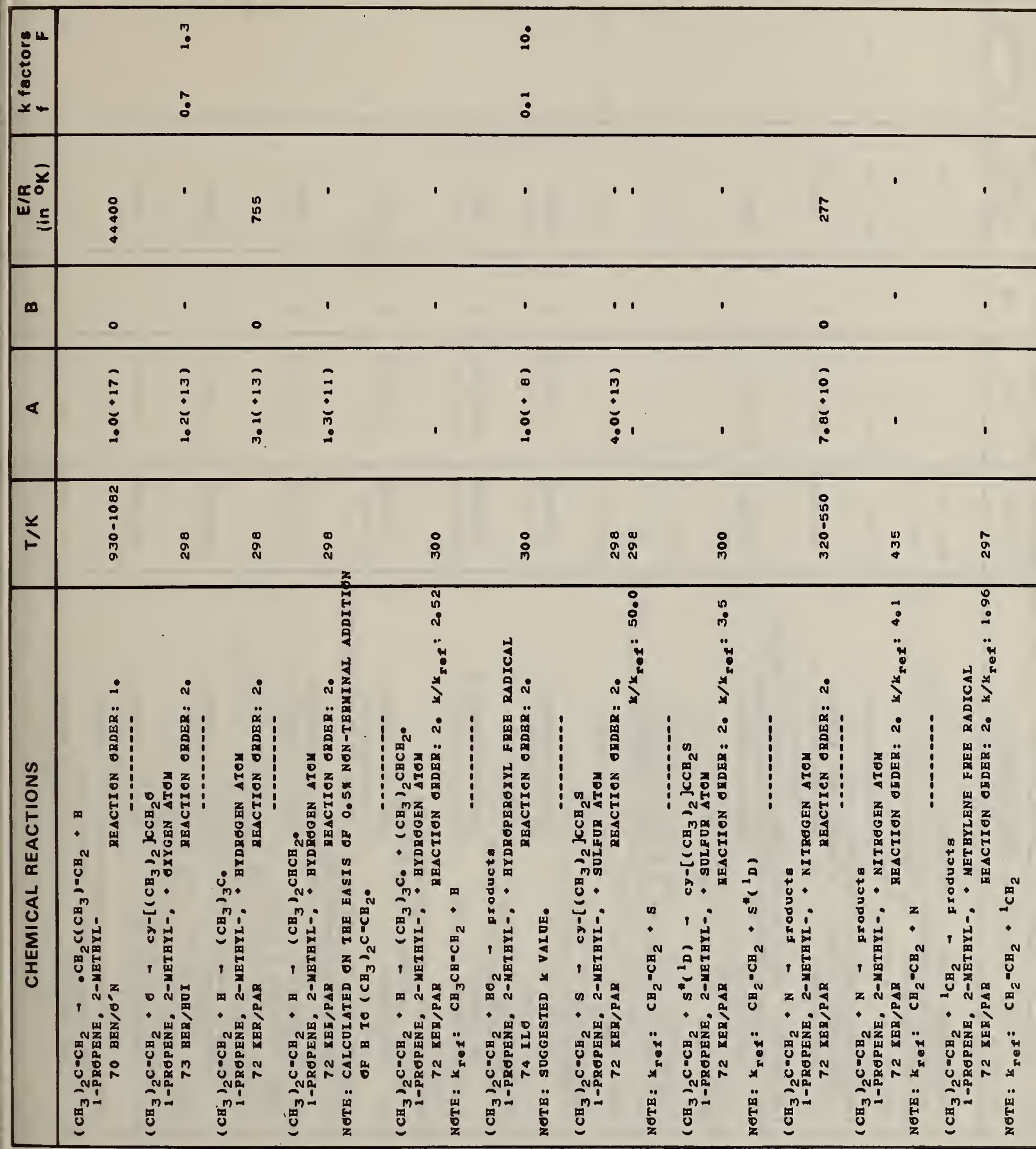




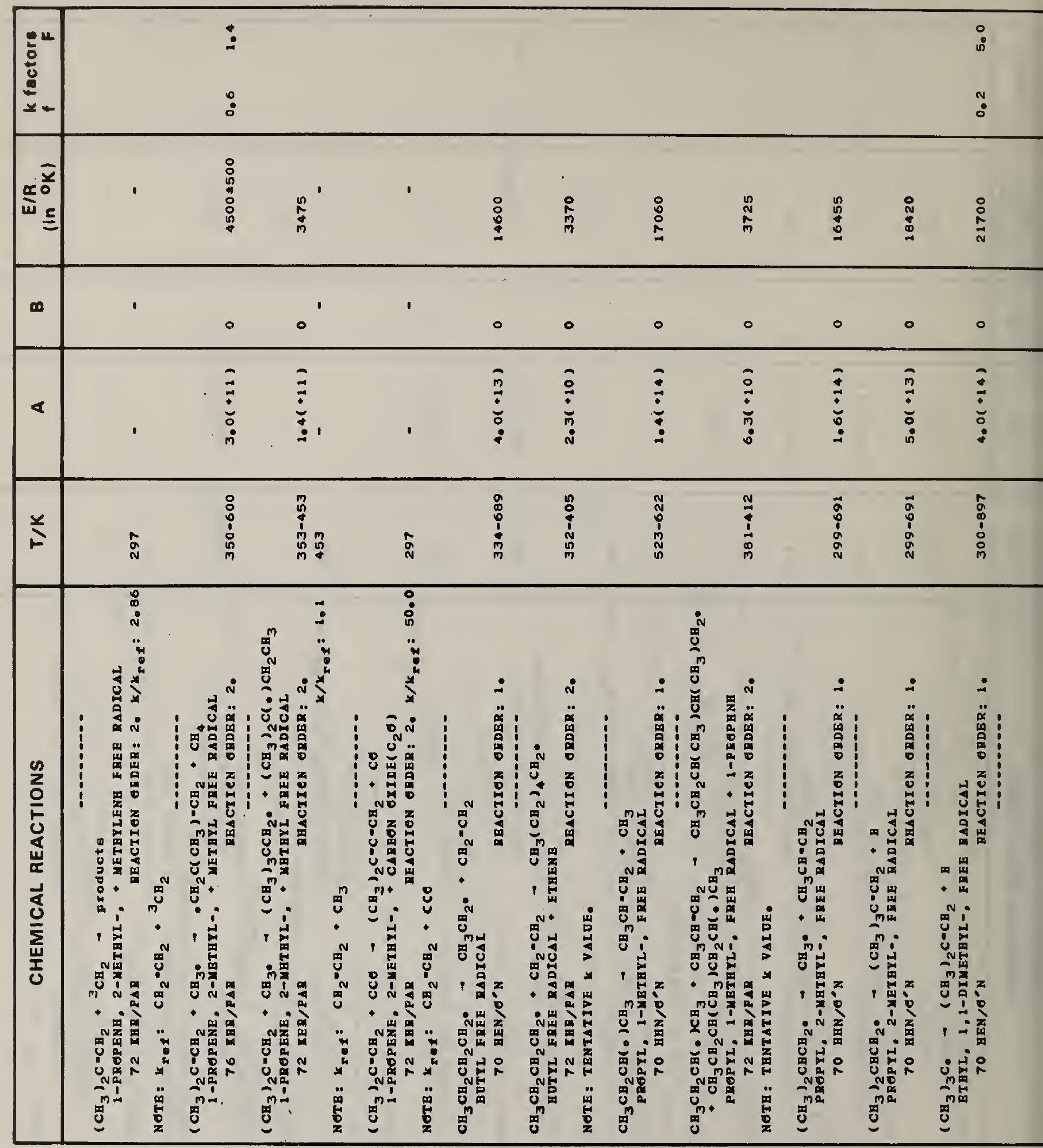




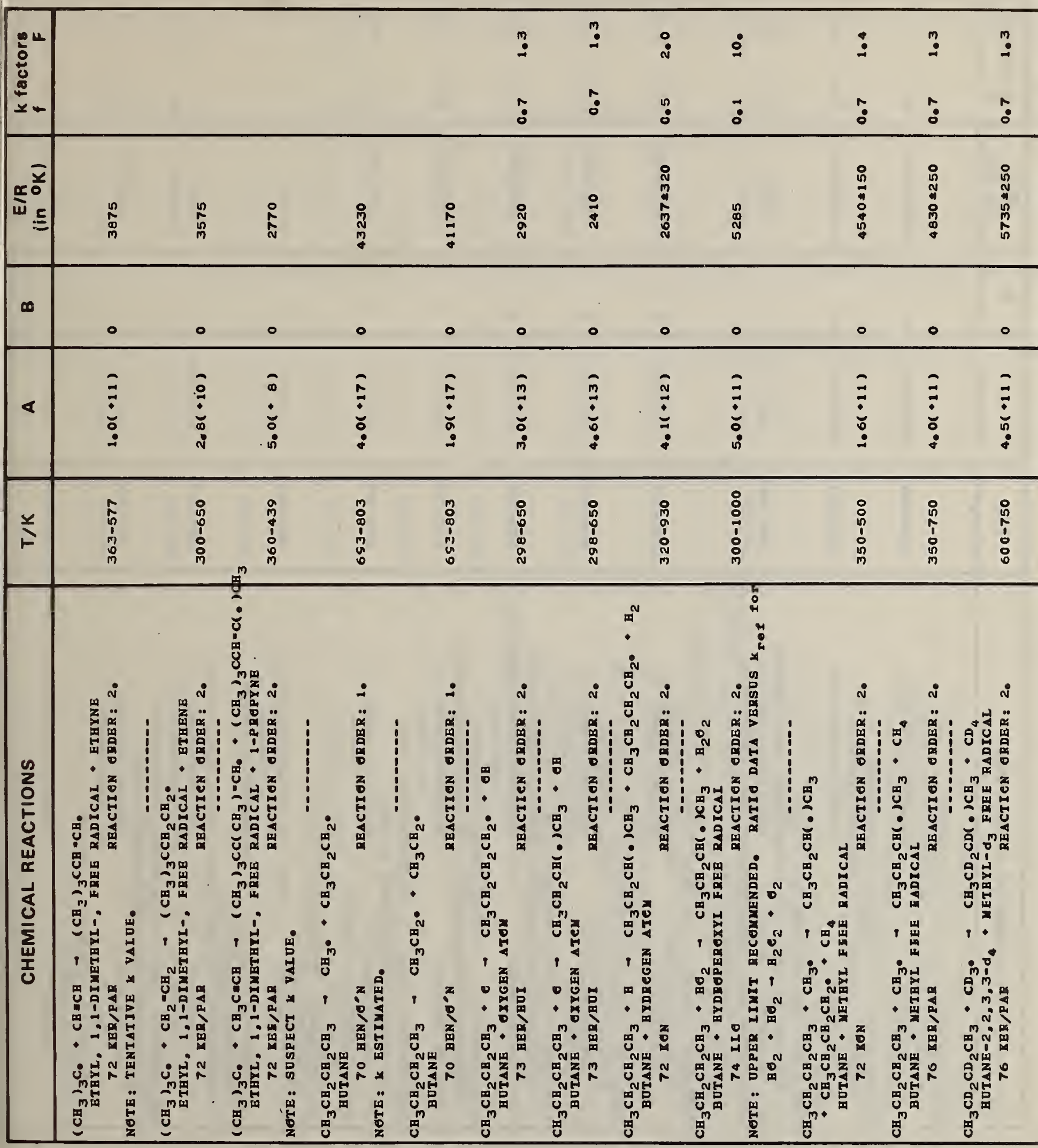




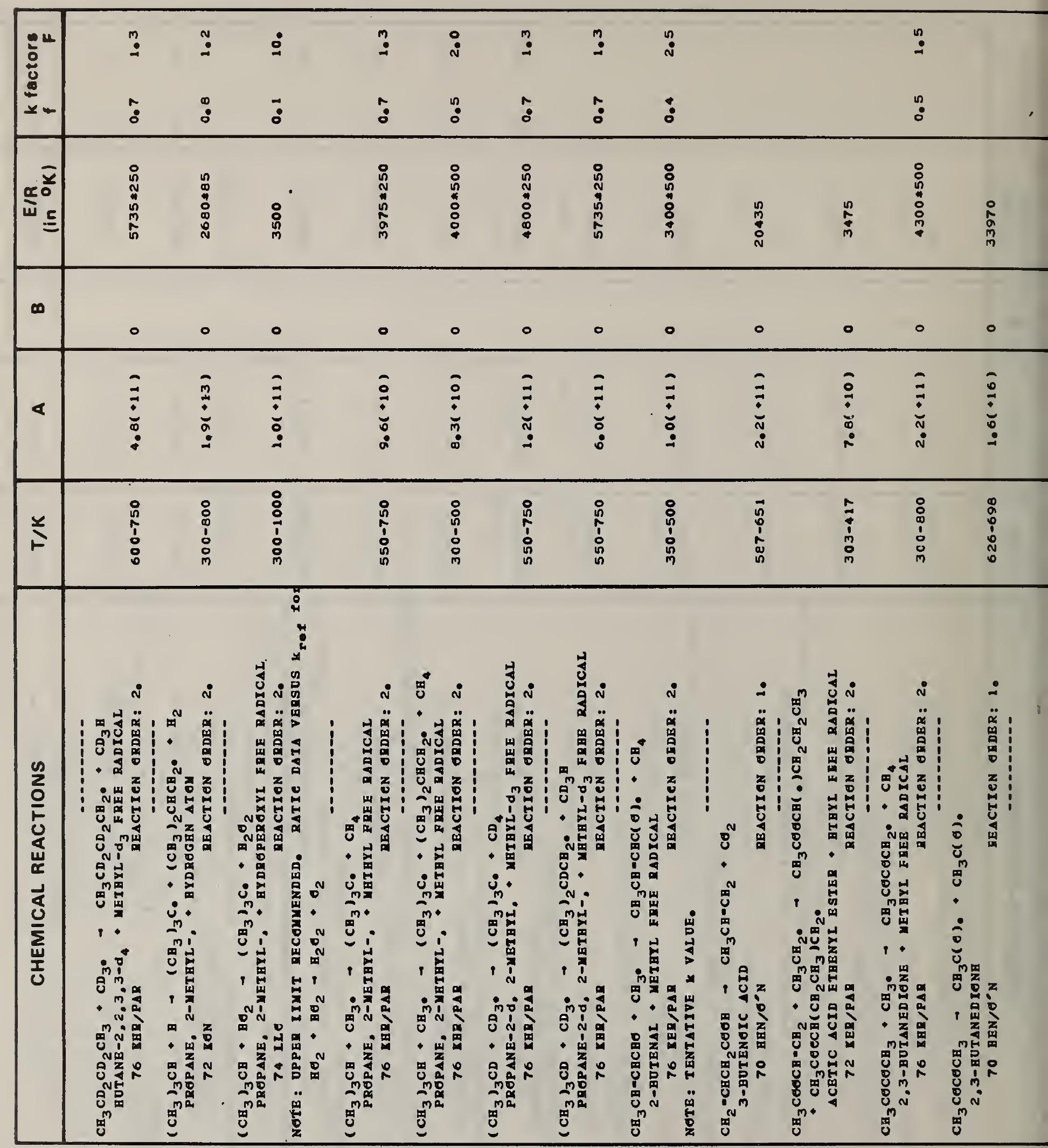




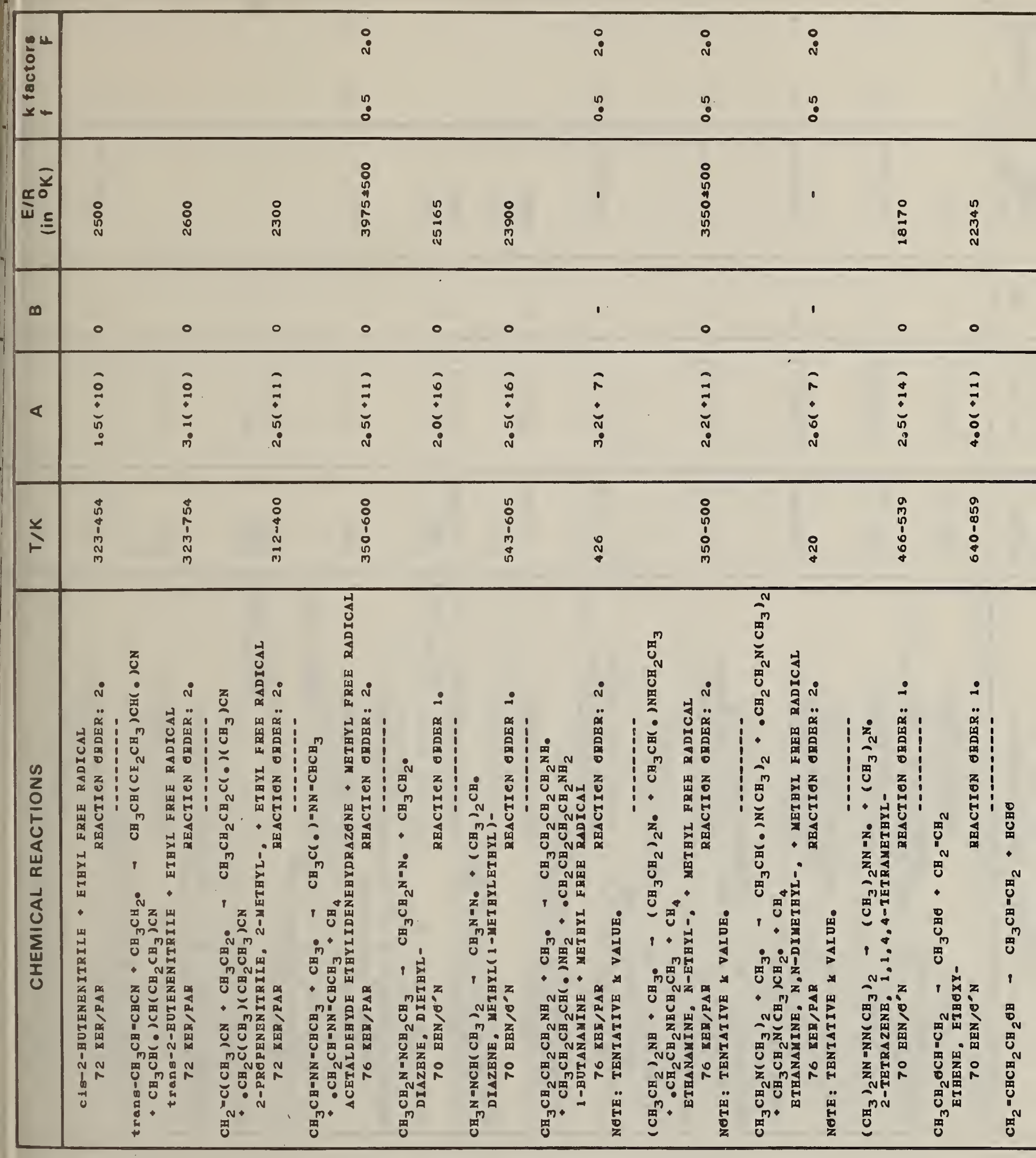




\begin{tabular}{|c|c|c|c|c|c|c|c|c|c|c|c|c|c|c|c|c|c|c|c|c|c|c|c|}
\hline $\begin{array}{l}\frac{1}{0} \\
0 \\
0 \\
x\end{array}$ & & & $\begin{array}{l}0 \\
:\end{array}$ & $:$ & : & $\begin{array}{l}? \\
? \\
?\end{array}$ & & $\begin{array}{l}n \\
: \\
?\end{array}$ & & & & & & & $\begin{array}{l}\because \\
\because \\
\because\end{array}$ & & & & & & & $\begin{array}{l}: \\
:\end{array}$ & \\
\hline$\underbrace{\frac{x}{0}}$ & $\begin{array}{l}\stackrel{n}{n} \\
\text { \& } \\
\stackrel{D}{0}\end{array}$ & & $\begin{array}{l}8 \\
8 \\
n \\
\hat{n} \\
0 \\
0 \\
\alpha \\
N\end{array}$ & $\begin{array}{l}8 \\
0 \\
11 \\
0 \\
o \\
\text { N }\end{array}$ & 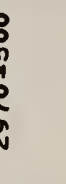 & $\begin{array}{l}0 \\
8 \\
0 \\
\text { Hै } \\
\text { ñ } \\
\text { - }\end{array}$ & & 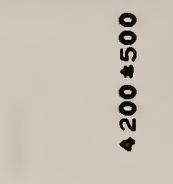 & & $\begin{array}{l}: \\
: \\
:\end{array}$ & & $\overbrace{}^{\prime}$ & & & ' & & 11 & & & I & & $\begin{array}{l}8 \\
8 \\
10 \\
0 \\
0 \\
8 \\
0 \\
0\end{array}$ & \\
\hline $\boldsymbol{\infty}$ & - & & 0 & ○ & ? & 0 & & 0 & & 0 & & $0^{\prime}$ & & & ' & & 11 & & & ' & & 0 & \\
\hline$<$ & $\begin{array}{l}= \\
\vdots \\
\vdots\end{array}$ & & $\begin{array}{l}5 \\
\vdots \\
\vdots\end{array}$ & $\begin{array}{l}\vdots \\
\vdots\end{array}$ & 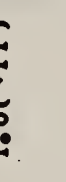 & $\begin{array}{l}\equiv \\
\vdots \\
\vdots\end{array}$ & & $\begin{array}{l}= \\
\vdots \\
\vdots\end{array}$ & & 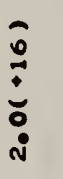 & & $\begin{array}{l} \pm \\
\vdots \\
\vdots \\
m^{\circ}\end{array}$ & & & $\begin{array}{l}\vec{N} \\
\stackrel{+}{0} \\
\dot{\infty} \\
\dot{v}\end{array}$ & & $\begin{array}{l}= \\
= \\
\bar{m} \\
\square\end{array}$ & & & $\begin{array}{l}\stackrel{N}{\vdots} \\
\vdots \\
\infty\end{array}$ & & $\begin{array}{l}\equiv \\
\vdots \\
\square\end{array}$ & \\
\hline$\underline{1}$ & $\begin{array}{l}\text { n } \\
\mathbf{0} \\
0 \\
1 \\
0 \\
m \\
0\end{array}$ & & $\begin{array}{l}: \\
: \\
1 \\
0 \\
0 \\
n \\
m\end{array}$ & $\begin{array}{l}: \\
: \\
1 \\
\text { o } \\
\text { in }\end{array}$ & ? & $\begin{array}{l}0 \\
: \\
0 \\
1 \\
0 \\
10 \\
m\end{array}$ & & $\begin{array}{l}8 \\
8 \\
0 \\
1 \\
\text { ํ } \\
m\end{array}$ & & 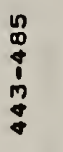 & & 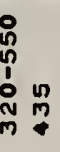 & & & $\stackrel{\infty}{\alpha}$ & & $\begin{array}{l}\infty \\
\stackrel{\infty}{N} \\
\sim\end{array}$ & & & 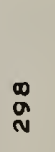 & & $\begin{array}{l}\text { 웅 } \\
0 \\
\vdots \\
0 \\
8\end{array}$ & \\
\hline 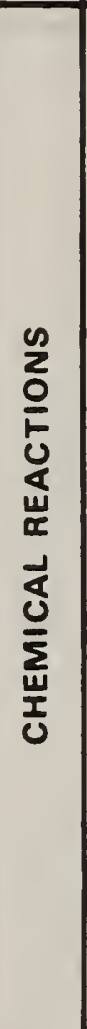 & 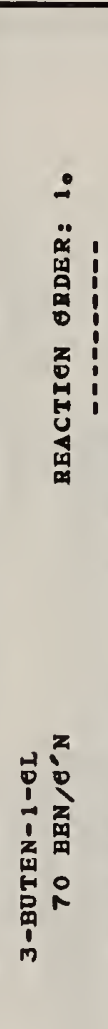 & 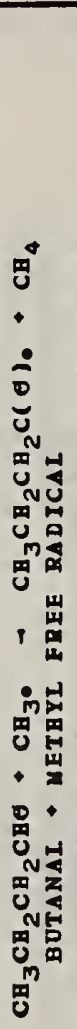 & 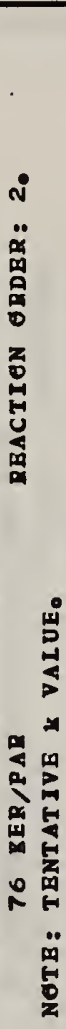 & 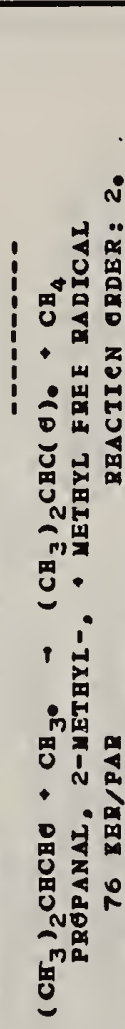 & 要 & 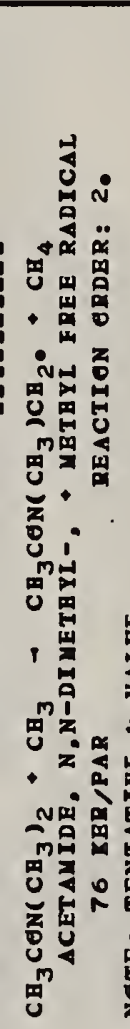 & 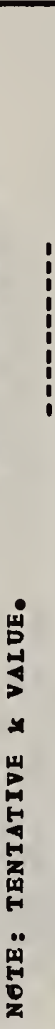 & $\begin{array}{l}\text { in } \\
\text { ! }\end{array}$ & 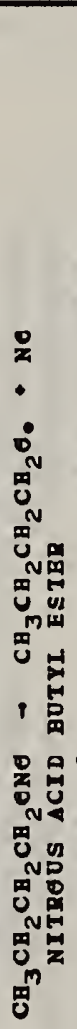 & 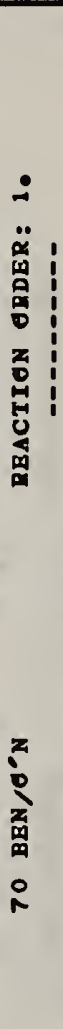 & 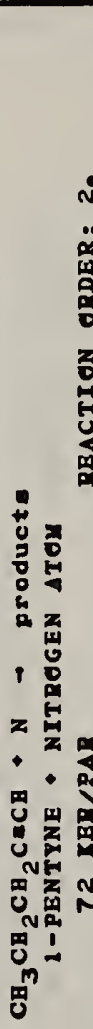 & D & 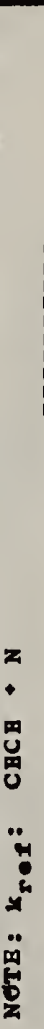 & 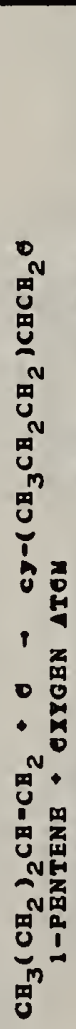 & 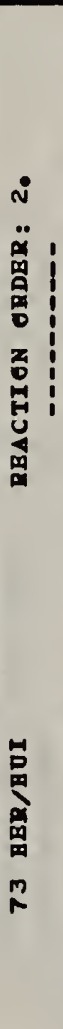 & 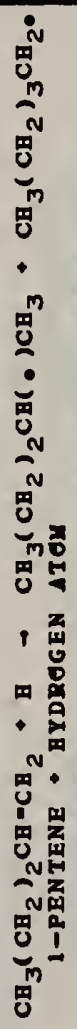 & 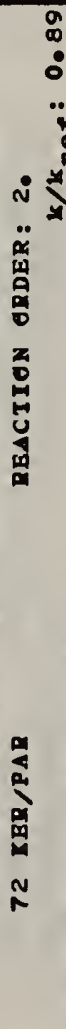 & 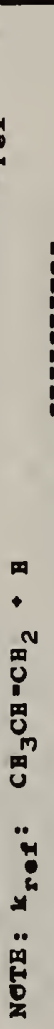 & 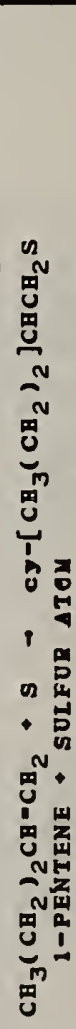 & 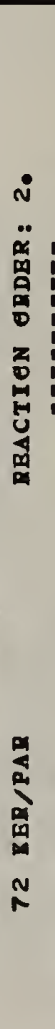 & 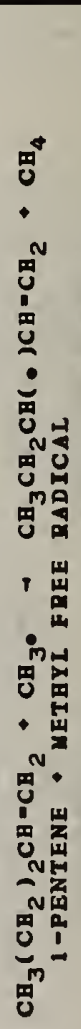 & 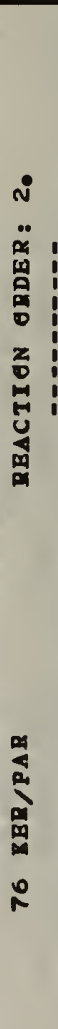 & 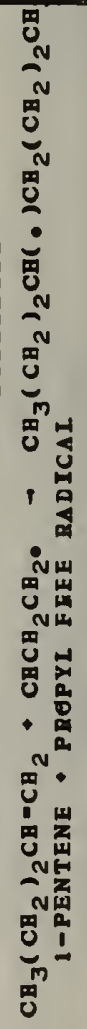 \\
\hline
\end{tabular}




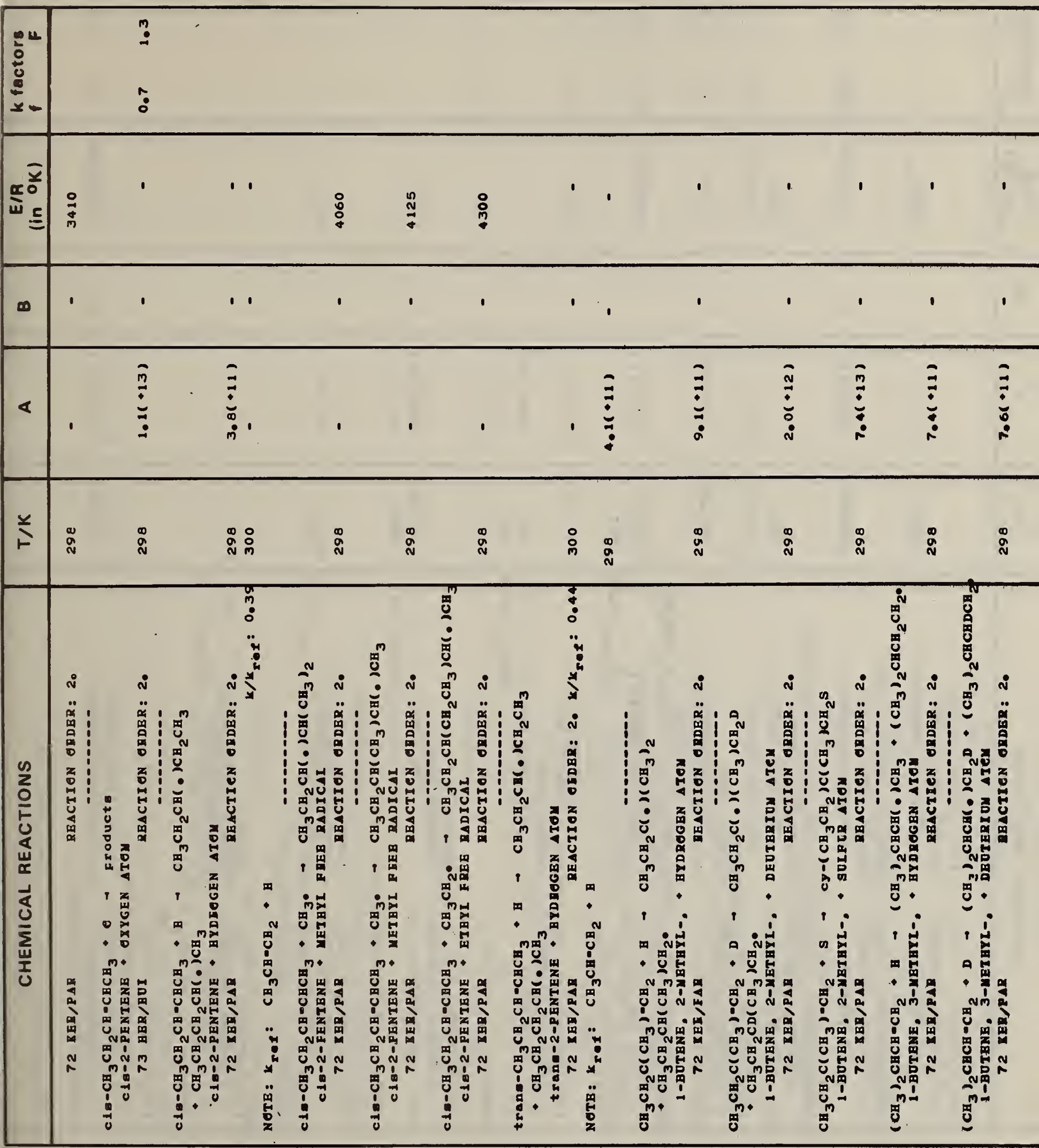




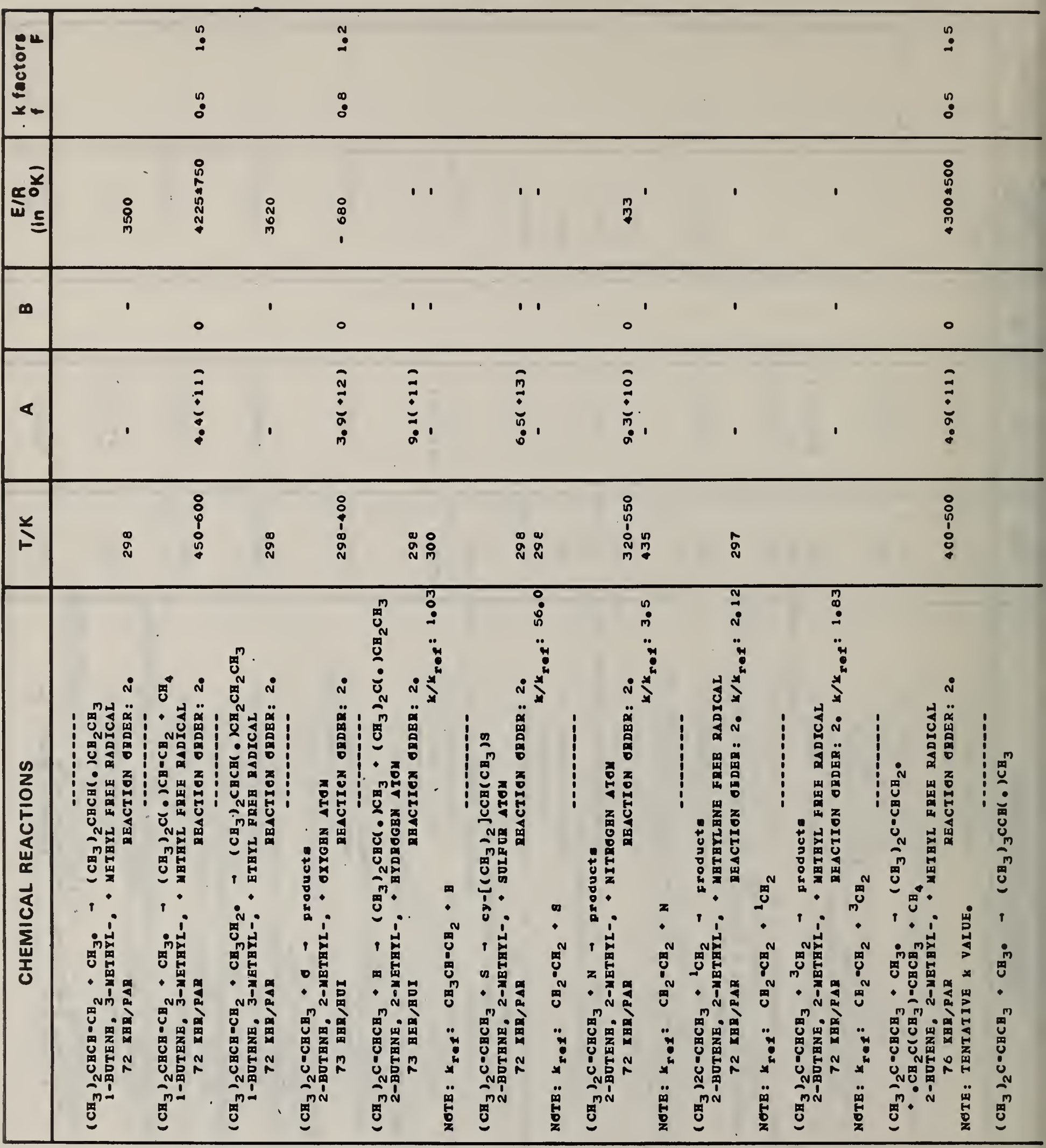




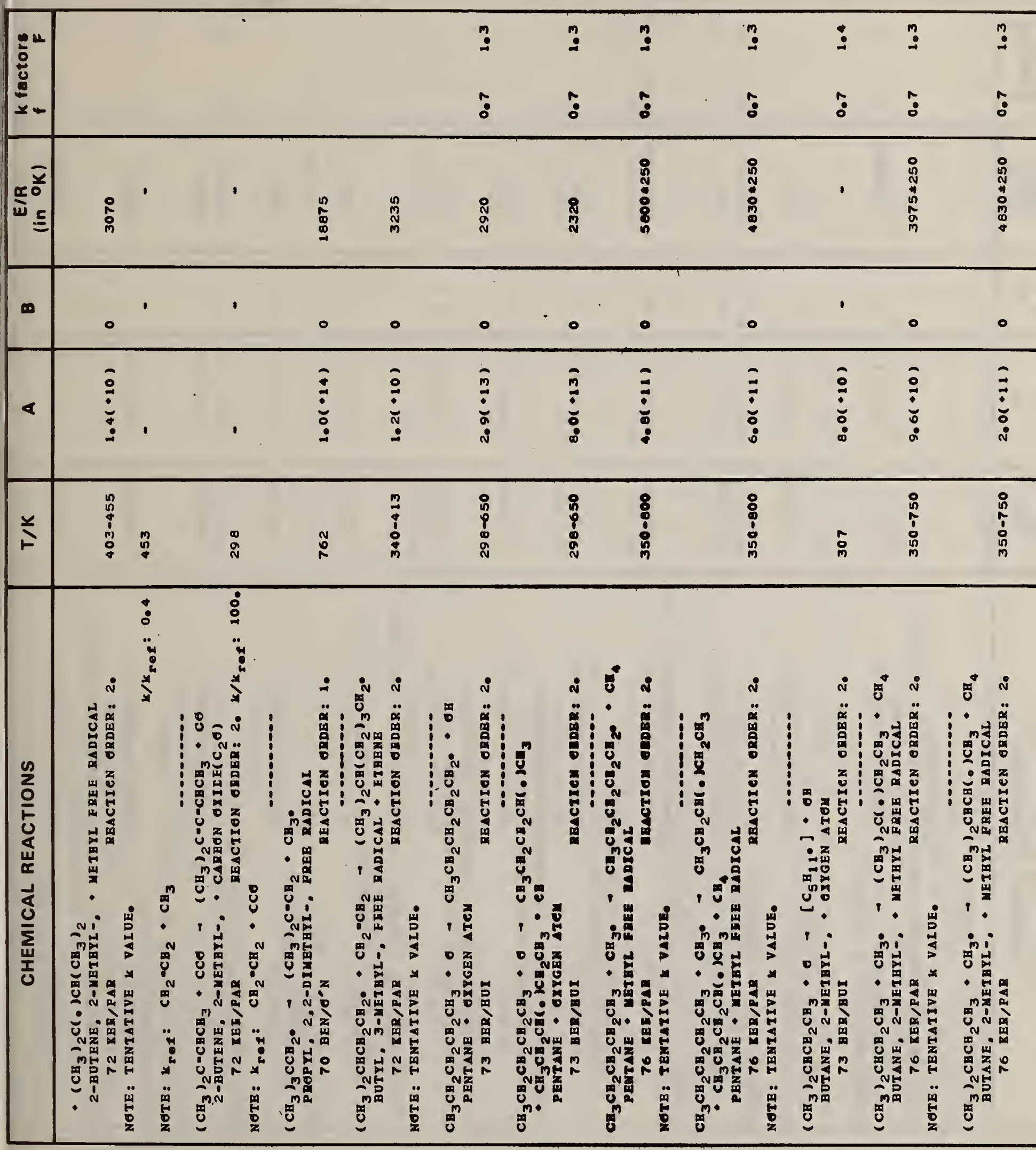




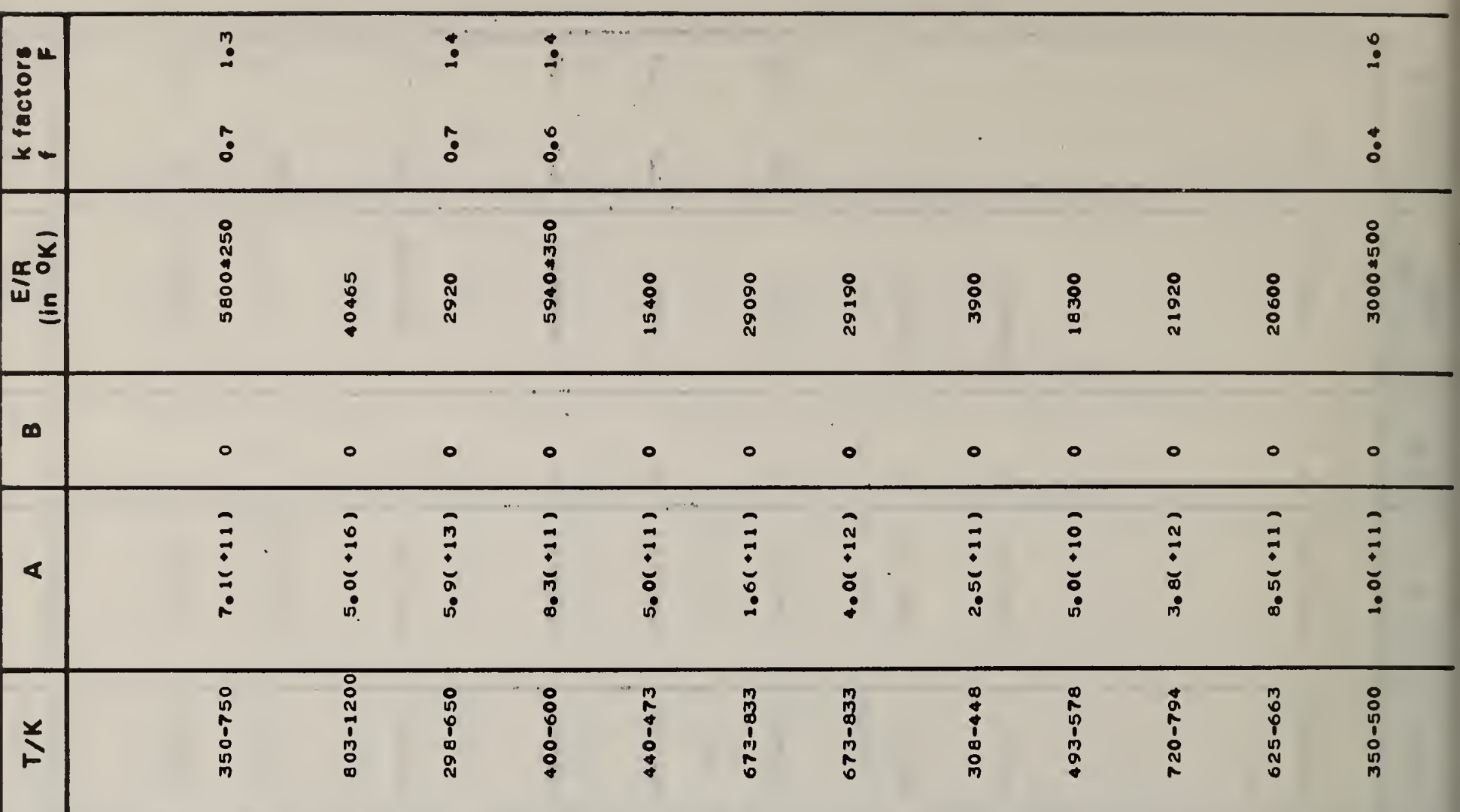

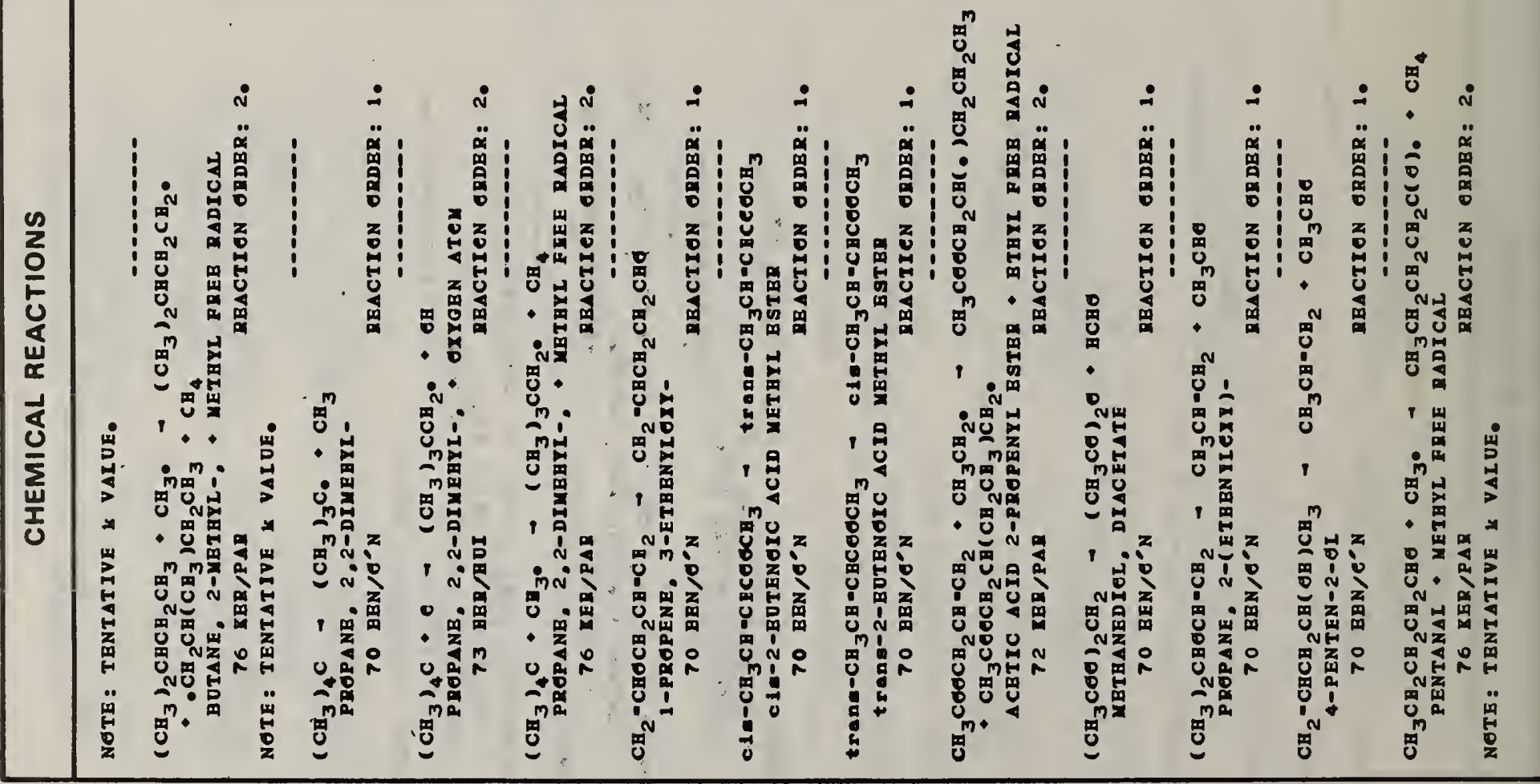




\begin{tabular}{|c|c|c|c|c|c|c|c|c|c|c|c|}
\hline$\square^{\prime \prime}$ & $\stackrel{0}{\dot{\sim}}$ & $\because$ & : & $\stackrel{?}{?}$ & $\dot{0}$ & : & $\stackrel{n}{\sim}$ & : & & & \\
\hline$\frac{x}{x-}$ & 0 & $\stackrel{+}{:}$ & $\stackrel{n}{\circ}$ & $\stackrel{n}{:}$ & $?$ & : & : & ! & & & \\
\hline$\frac{x}{\omega}^{\frac{\bar{x}}{0}}$ & $\begin{array}{l}: \\
: \\
0 \\
: 1 \\
: \\
\stackrel{N}{N} \\
m\end{array}$ & $\begin{array}{l}8 \\
0 \\
0 \\
\ddot{D} \\
0 \\
\vdots \\
0 \\
0\end{array}$ & $\begin{array}{l}8 \\
: \\
0 \\
0 \\
0 \\
m \\
m\end{array}$ & 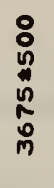 & $\begin{array}{l}8 \\
: \\
0 \\
00 \\
0 \\
000 \\
0 \\
0\end{array}$ & 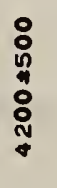 & 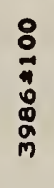 & $\begin{array}{l}8 \\
: \\
1 \\
\ddot{0} \\
0 \\
0 \\
0 \\
0\end{array}$ & \begin{tabular}{l}
$\stackrel{0}{m}$ \\
$\stackrel{m}{\alpha}$ \\
\hdashline
\end{tabular} & \begin{tabular}{l}
$:$ \\
$:$ \\
$\vdots$ \\
\multirow{+}{*}{}
\end{tabular} & \begin{tabular}{l}
$n$ \\
\multirow{d}{*}{} \\
N
\end{tabular} \\
\hline (๓) & 0 & ○ & - & 0 & 0 & 0 & 0 & 0 & 0 & 0 & 0 \\
\hline$<$ & $\stackrel{\square}{\Xi}$ & 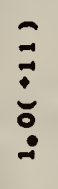 & $\begin{array}{l}\Xi \\
\vdots \\
\vdots\end{array}$ & $\begin{array}{l}\bar{\Xi} \\
\vdots \\
\vdots\end{array}$ & $\begin{array}{l}\bar{\Xi} \\
\dot{\vdots}\end{array}$ & $\bar{\Xi}$ & $\underset{\Xi}{\Xi}$ & 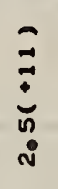 & 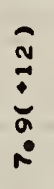 & 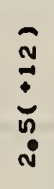 & $\stackrel{n}{n}$ \\
\hline$\stackrel{Y}{F}$ & $\begin{array}{l}0 \\
: \\
0 \\
1 \\
\vdots \\
0 \\
1\end{array}$ & $\begin{array}{l}: \\
: \\
1 \\
0 \\
0 \\
n \\
n\end{array}$ & $\begin{array}{l}8 \\
: \\
1 \\
1 \\
0 \\
n \\
m\end{array}$ & $\begin{array}{l}8 \\
i \\
i \\
0 \\
0 \\
m\end{array}$ & $\begin{array}{l}: \\
\vdots \\
\vdots \\
\vdots\end{array}$ & $\begin{array}{l}: \\
\vdots \\
\vdots \\
\vdots \\
\vdots \\
i\end{array}$ & $\begin{array}{l}\stackrel{N}{N} \\
1 \\
\vdots \\
\circ \\
⿱ 亠 凶\end{array}$ & $\begin{array}{l}8 \\
0 \\
1 \\
0 \\
0 \\
n\end{array}$ & \begin{tabular}{l}
$m$ \\
0 \\
0 \\
1 \\
$n !$ \\
0 \\
\hdashline
\end{tabular} & $\begin{array}{l}0 \\
\stackrel{-}{0} \\
\vdots \\
\stackrel{n}{N} \\
N\end{array}$ & $\begin{array}{l}\overrightarrow{0} \\
\vdots \\
1 \\
0 \\
\vdots \\
n\end{array}$ \\
\hline
\end{tabular}

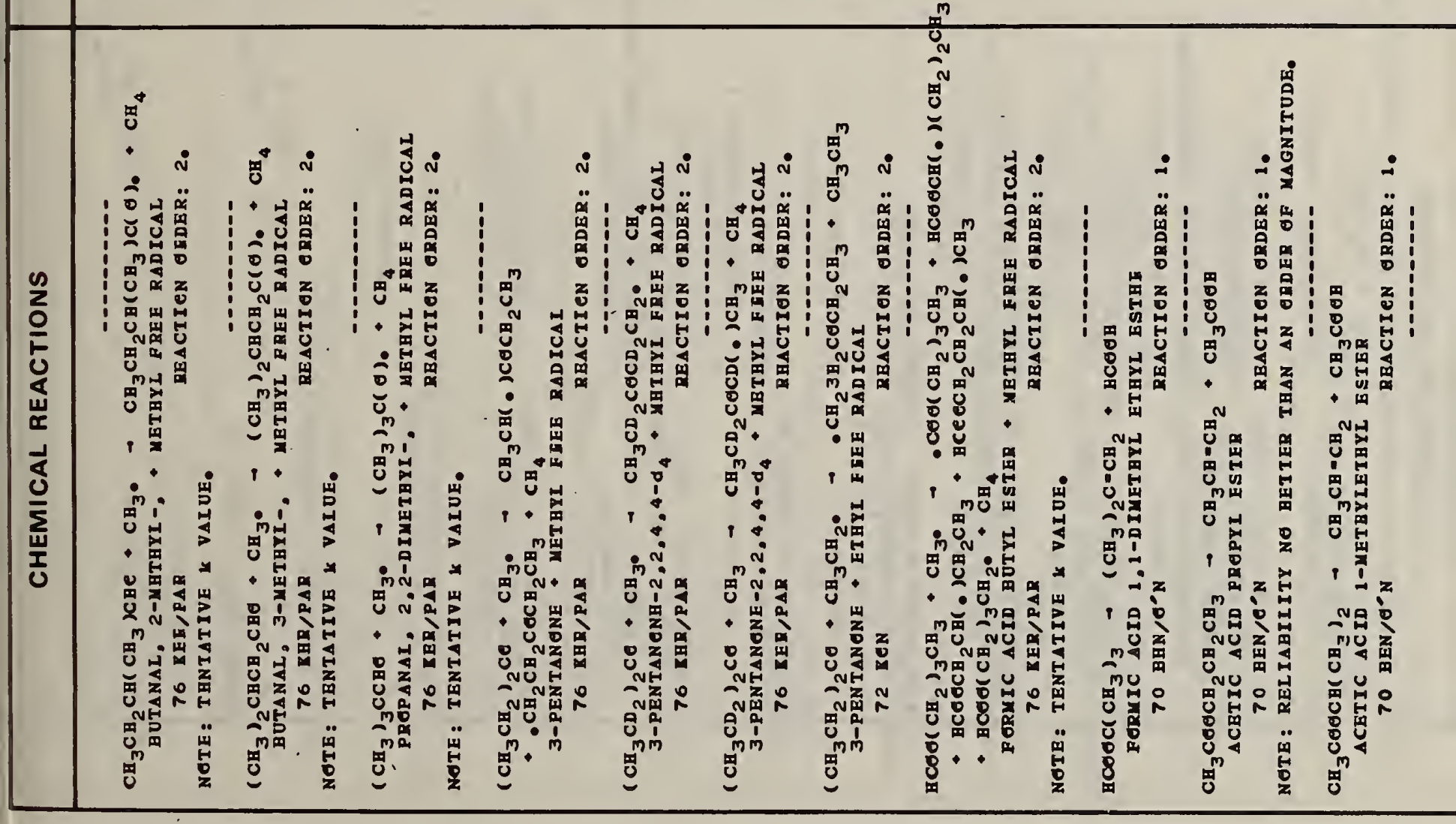




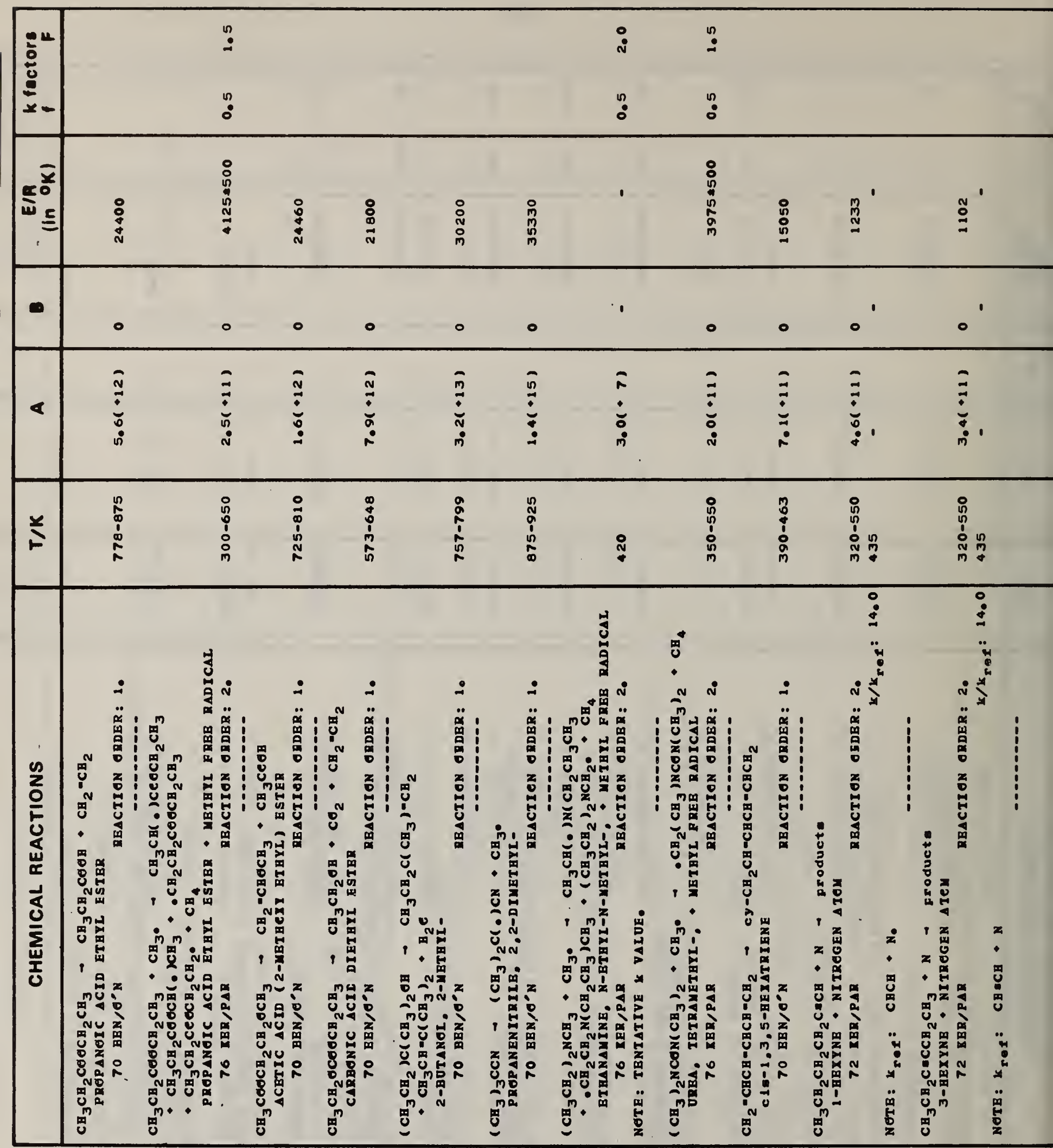




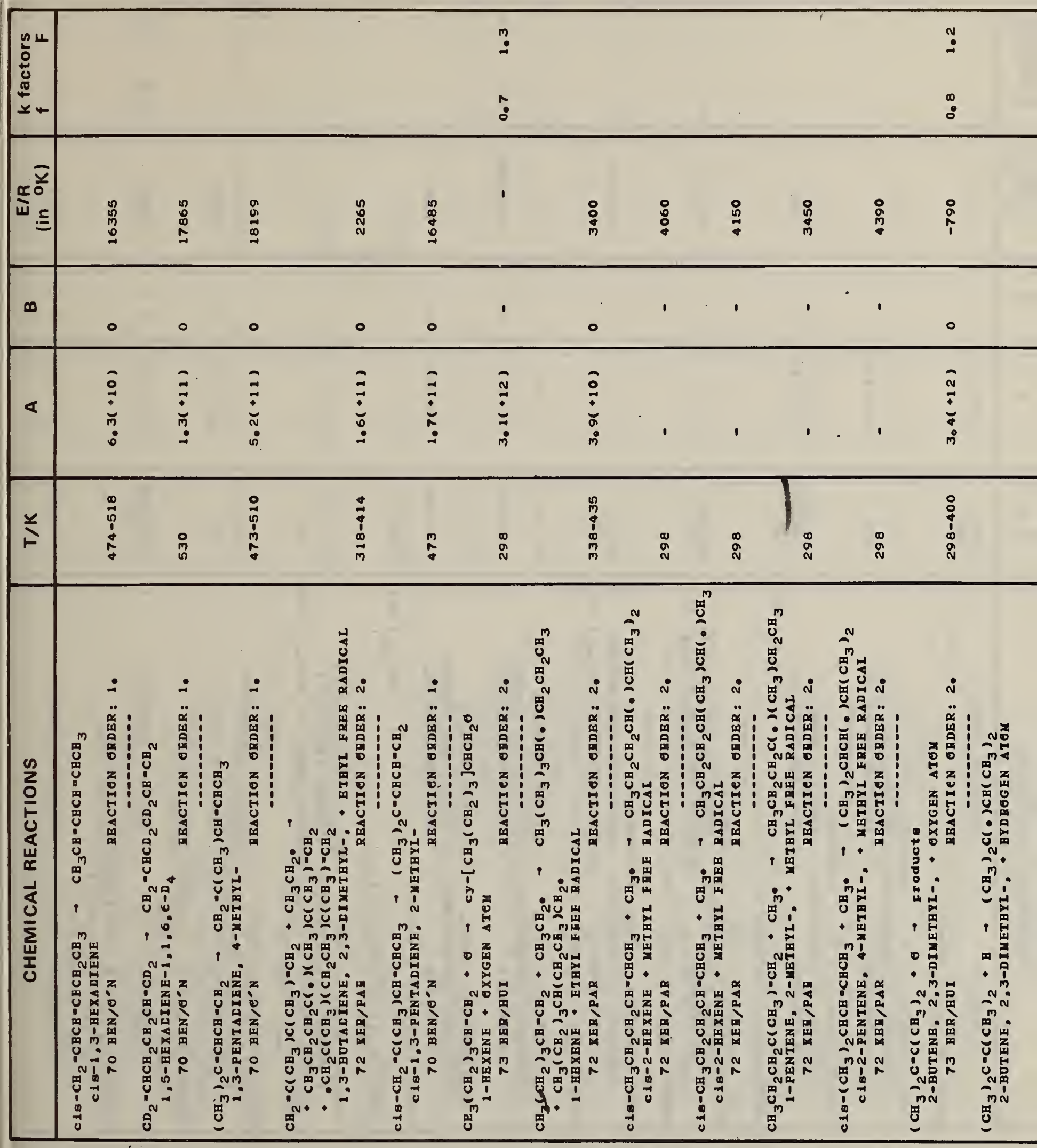




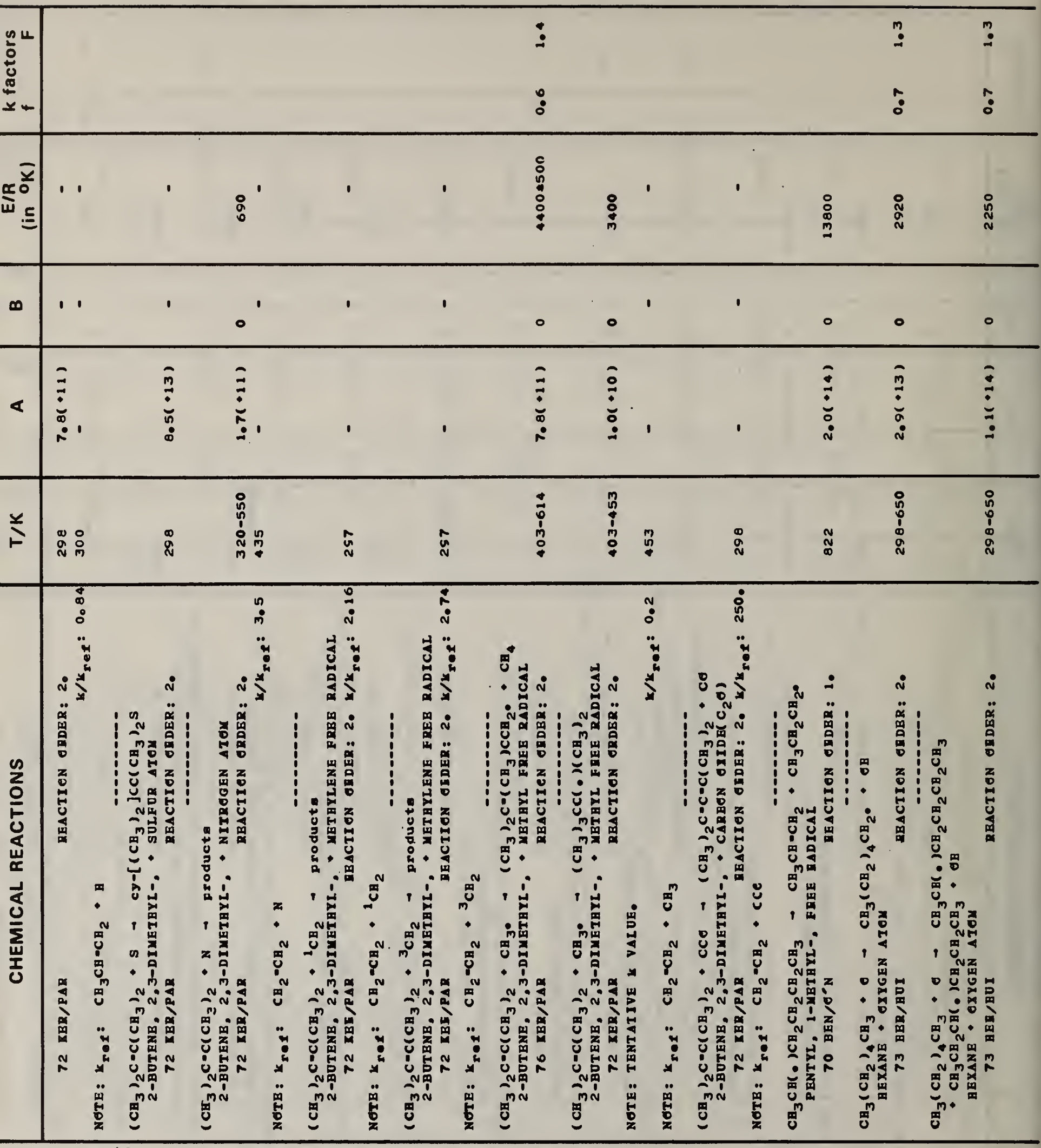




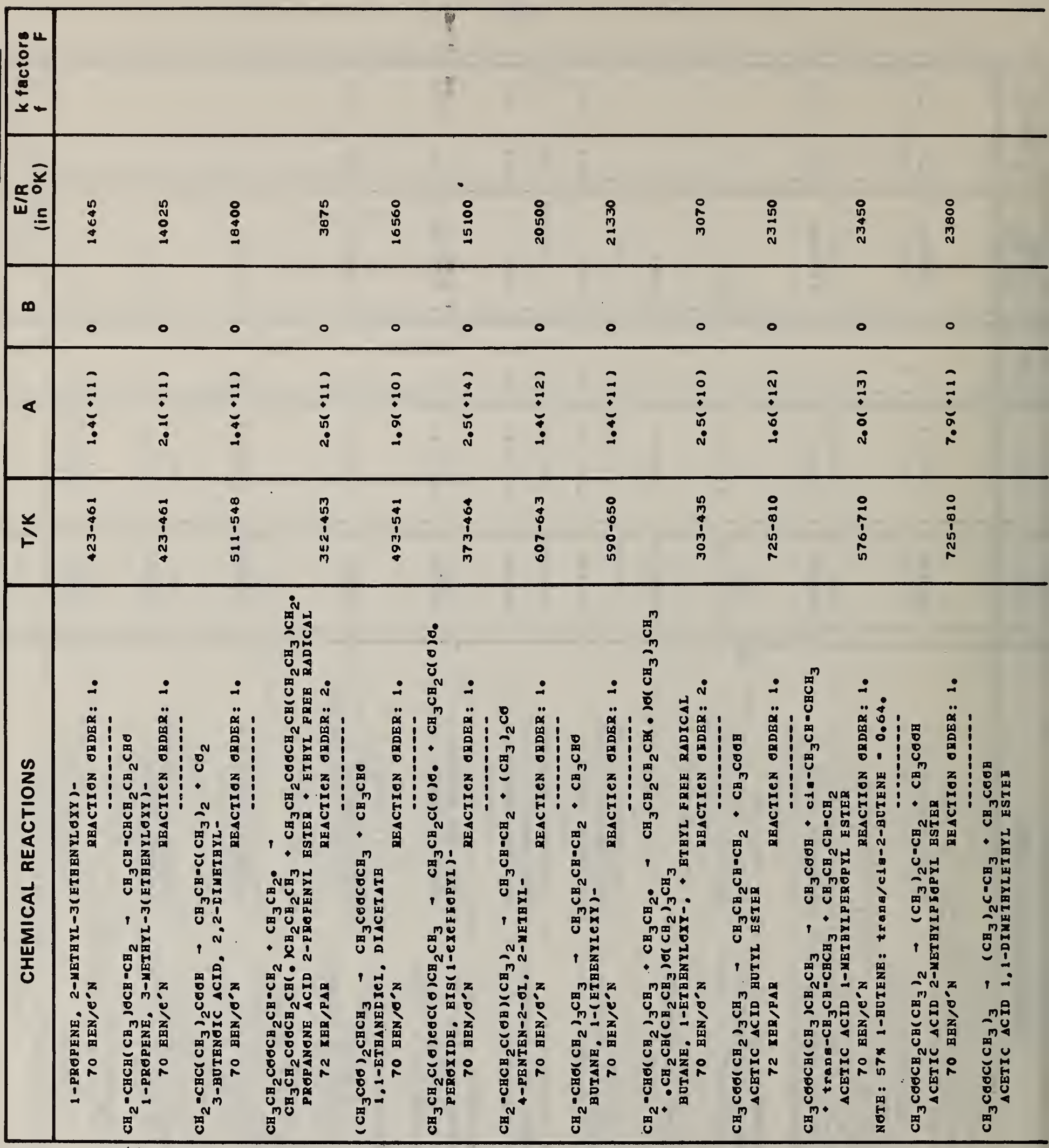




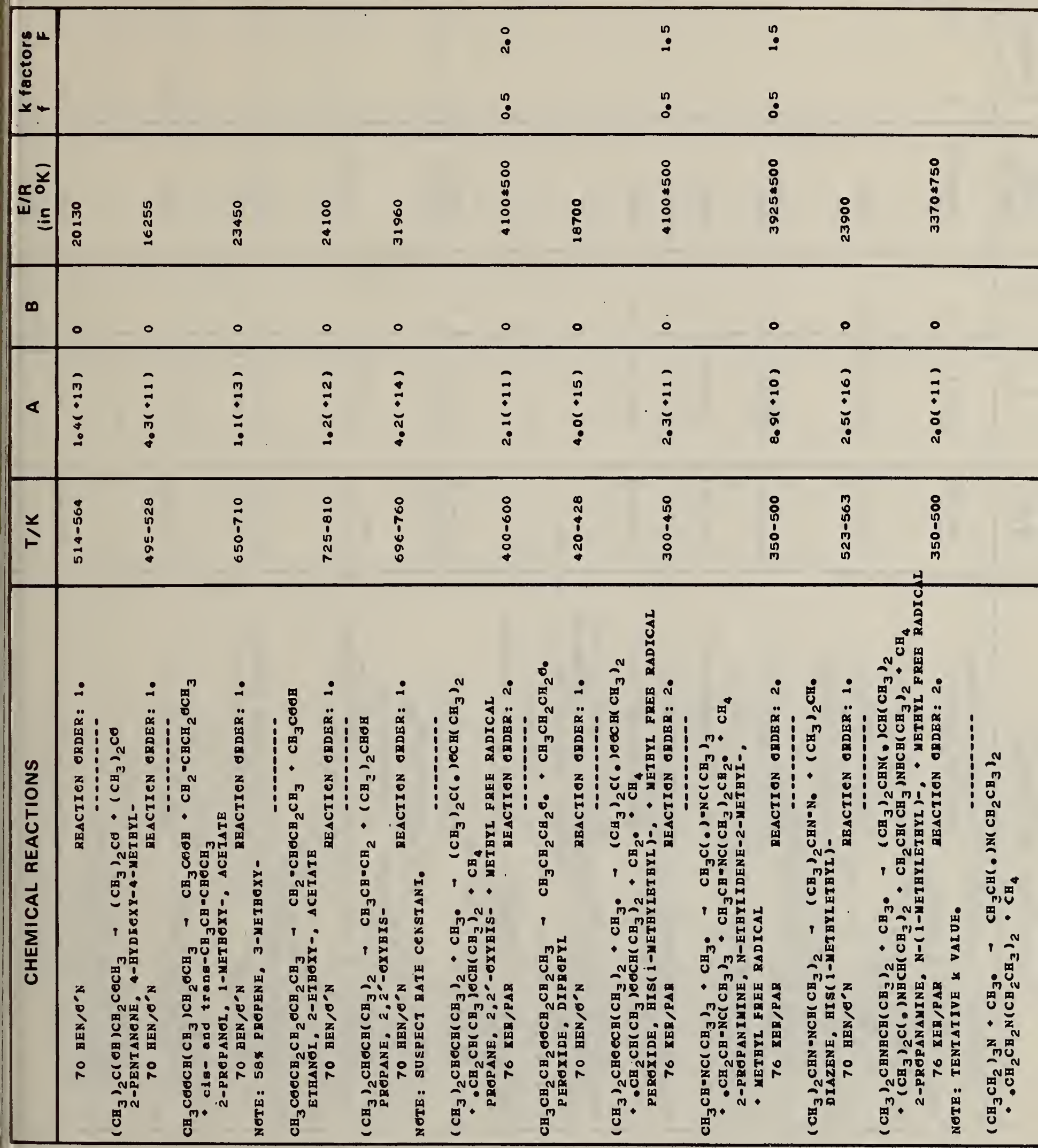




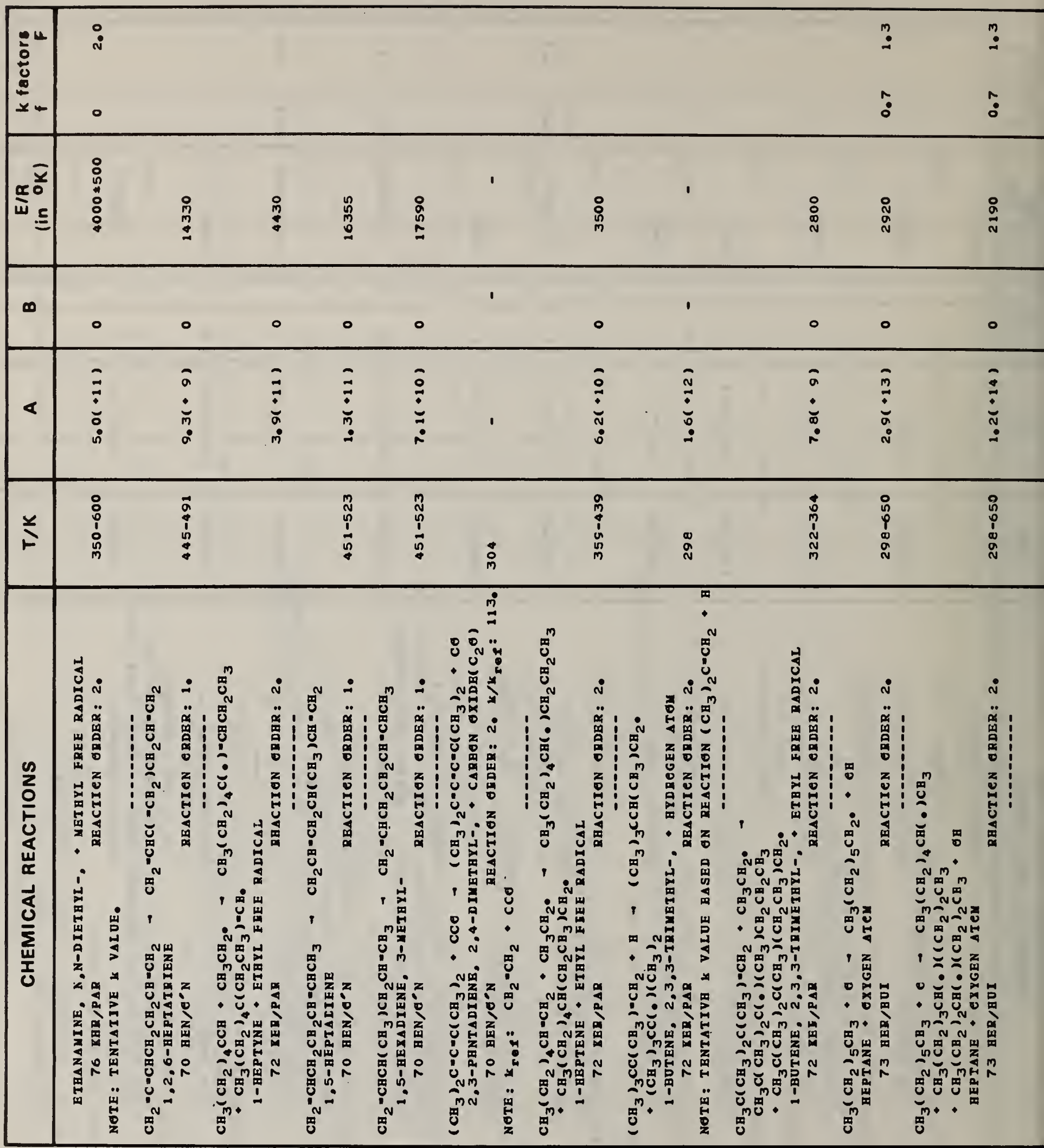




\begin{tabular}{|c|c|c|c|c|c|c|c|c|c|c|c|c|}
\hline 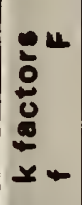 & $\stackrel{\circ}{\circ}$ & $\begin{array}{l}: \\
\therefore\end{array}$ & $\begin{array}{l}\because \\
\because\end{array}$ & $\begin{array}{l}m \\
\because \\
\therefore\end{array}$ & $\begin{array}{l}: \\
:\end{array}$ & & & & & & & \\
\hline$x^{\frac{2}{0}}$ & I & 1 & $\begin{array}{l}0 \\
0 \\
N \\
10 \\
n \\
0 \\
0 \\
m\end{array}$ & 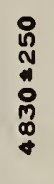 & $\begin{array}{l}\circ \\
\stackrel{8}{0} \\
4 \\
: \\
8 \\
8 \\
0\end{array}$ & $\begin{array}{l}n \\
m \\
m \\
0 \\
m\end{array}$ & $\begin{array}{l}0 \\
0 \\
0 \\
0\end{array}$ & $\begin{array}{l}: \\
\stackrel{p}{p} \\
\stackrel{N}{0}\end{array}$ & $\begin{array}{l}\stackrel{n}{\dot{*}} \\
\stackrel{N}{N} \\
\text { N }\end{array}$ & $\begin{array}{l}0 \\
: \\
\infty \\
\infty \\
\infty \\
-7\end{array}$ & 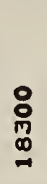 & $\begin{array}{l}\text { ㅇ } \\
\text { M } \\
\text { p } \\
\text { N }\end{array}$ \\
\hline$\infty$ & 1 & 1 & 0 & 0 & 0 & 0 & 0 & 0 & 0 & 0 & 0 & 0 \\
\hline$\varangle$ & $\begin{array}{l}0 \\
0 \\
\vdots \\
0\end{array}$ & $\begin{array}{l}= \\
\vdots \\
\vdots\end{array}$ & $\begin{array}{l}\hat{0} \\
\vdots \\
\text { ñ } \\
0\end{array}$ & $\begin{array}{l}\overrightarrow{1} \\
\vdots \\
0\end{array}$ & $\begin{array}{l}= \\
\vdots \\
=\end{array}$ & $\begin{array}{l}0 \\
\vdots \\
\vdots\end{array}$ & $\begin{array}{l}0 \\
\vdots \\
\vdots \\
0\end{array}$ & $\begin{array}{l}\vec{y} \\
\dot{0} \\
\text { in }\end{array}$ & $\begin{array}{l}\tilde{m} \\
\vdots \\
0\end{array}$ & $\begin{array}{l}\Xi \\
\vdots \\
\vdots \\
\vdots\end{array}$ & $\begin{array}{l}0 \\
\dot{0} \\
\dot{0} \\
\text { i }\end{array}$ & $\begin{array}{l}\vec{v} \\
\vdots \\
\vdots \\
-\end{array}$ \\
\hline$\underset{F}{F}$ & $\stackrel{\overrightarrow{0}}{\circ}$ & $\stackrel{M}{\circ}$ & $\begin{array}{l}\text { 음 } \\
\text { î } \\
\text { o } \\
\text { in }\end{array}$ & $\begin{array}{l}0 \\
\stackrel{n}{2} \\
1 \\
0 \\
0 \\
n\end{array}$ & $\begin{array}{l}\text { : } \\
\text { ñ } \\
\text { : } \\
\text { ñ }\end{array}$ & $\begin{array}{l}n \\
a \\
-1 \\
0 \\
u \\
0 \\
0\end{array}$ & $\begin{array}{l}\infty \\
0 \\
\infty \\
\$ \\
\vdots \\
\$\end{array}$ & 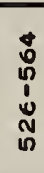 & $\begin{array}{l}\stackrel{\infty}{0} \\
\stackrel{0}{0} \\
\vdots \\
\dot{0} \\
0\end{array}$ & $\begin{array}{l}0 \\
\hat{n} \\
\text { in } \\
\text { un } \\
\text { N } \\
\text { in }\end{array}$ & 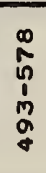 & $\begin{array}{l}0 \\
\stackrel{0}{\infty} \\
1 \\
1 \\
N \\
N\end{array}$ \\
\hline
\end{tabular}

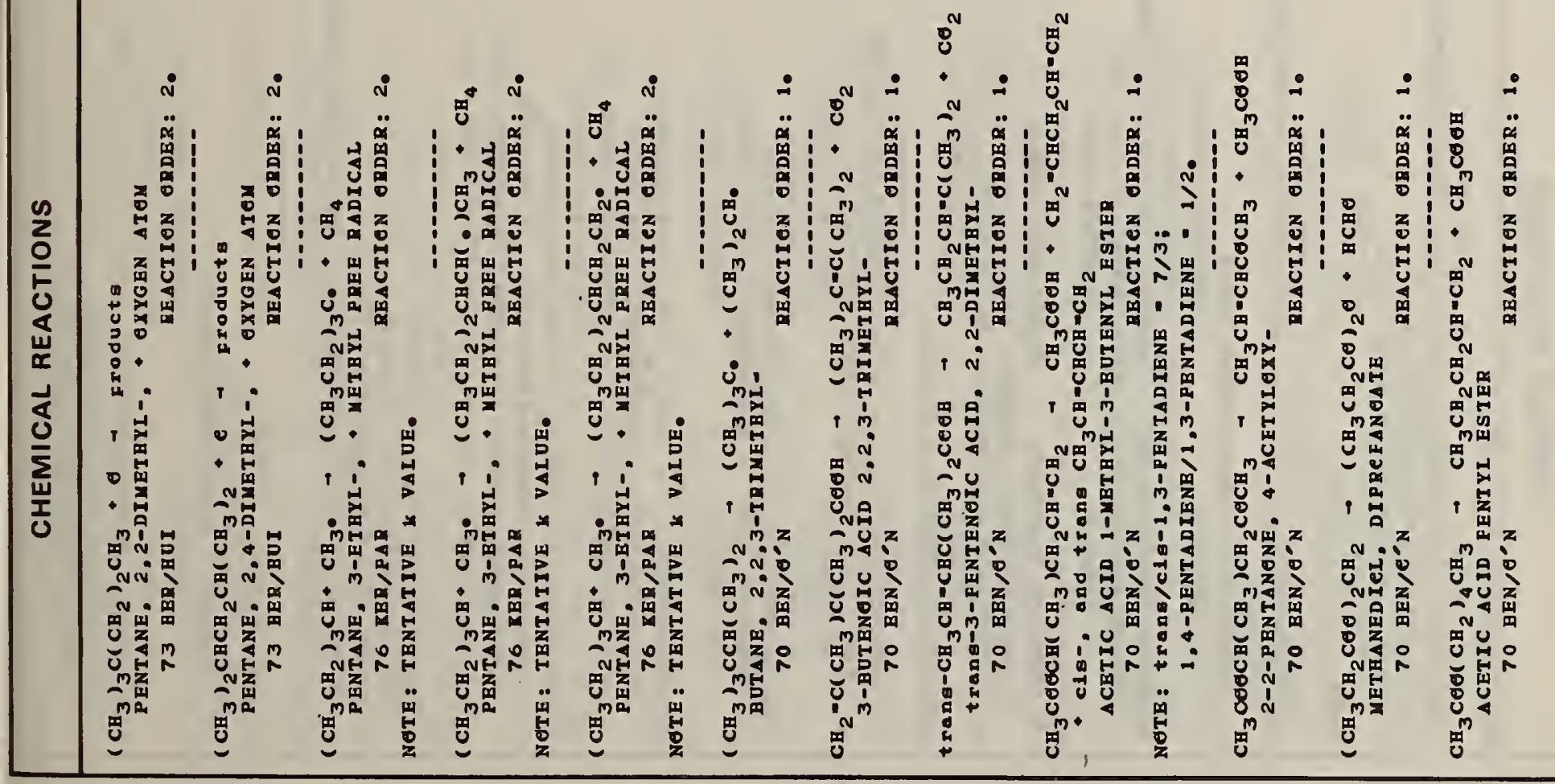




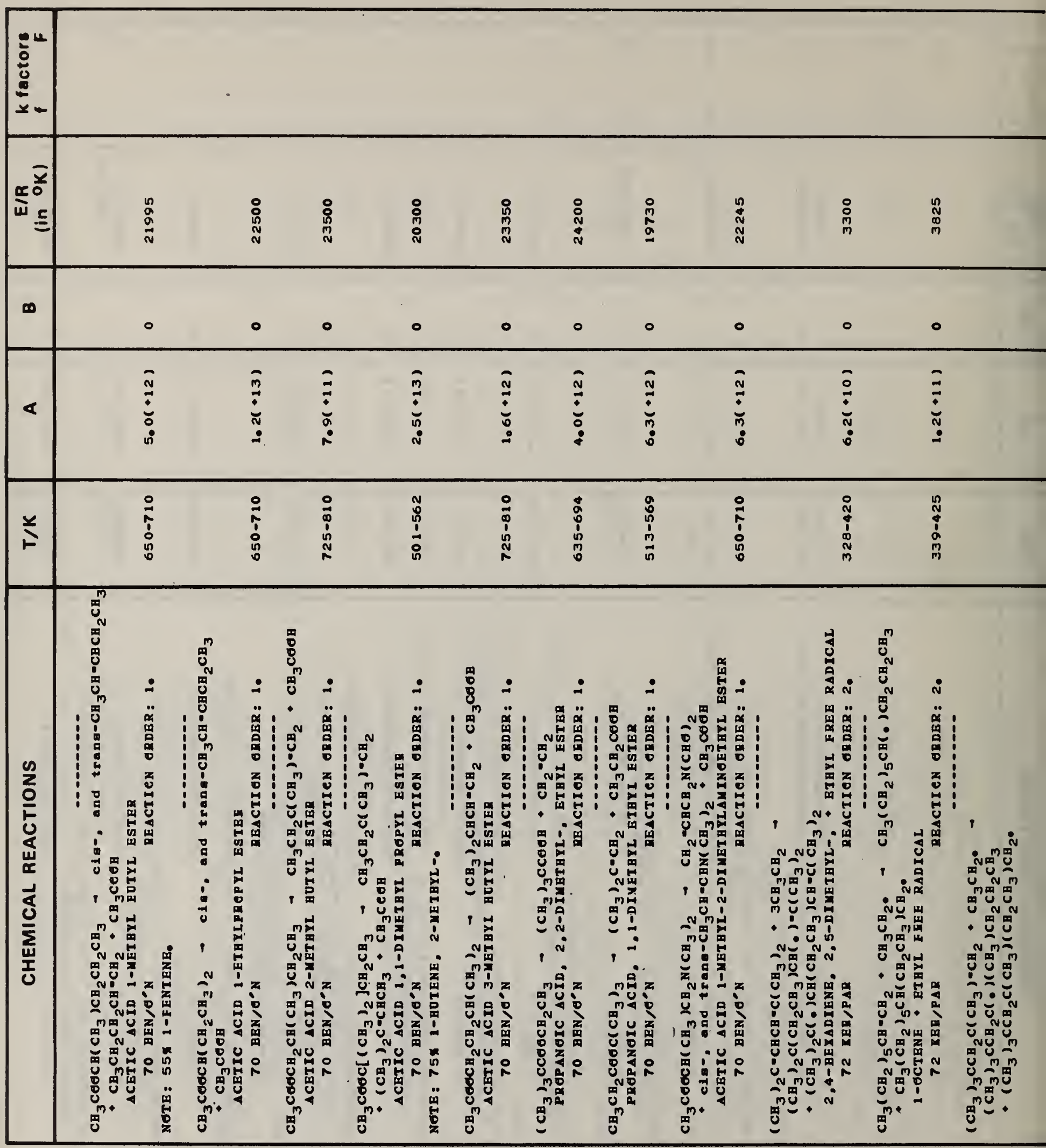




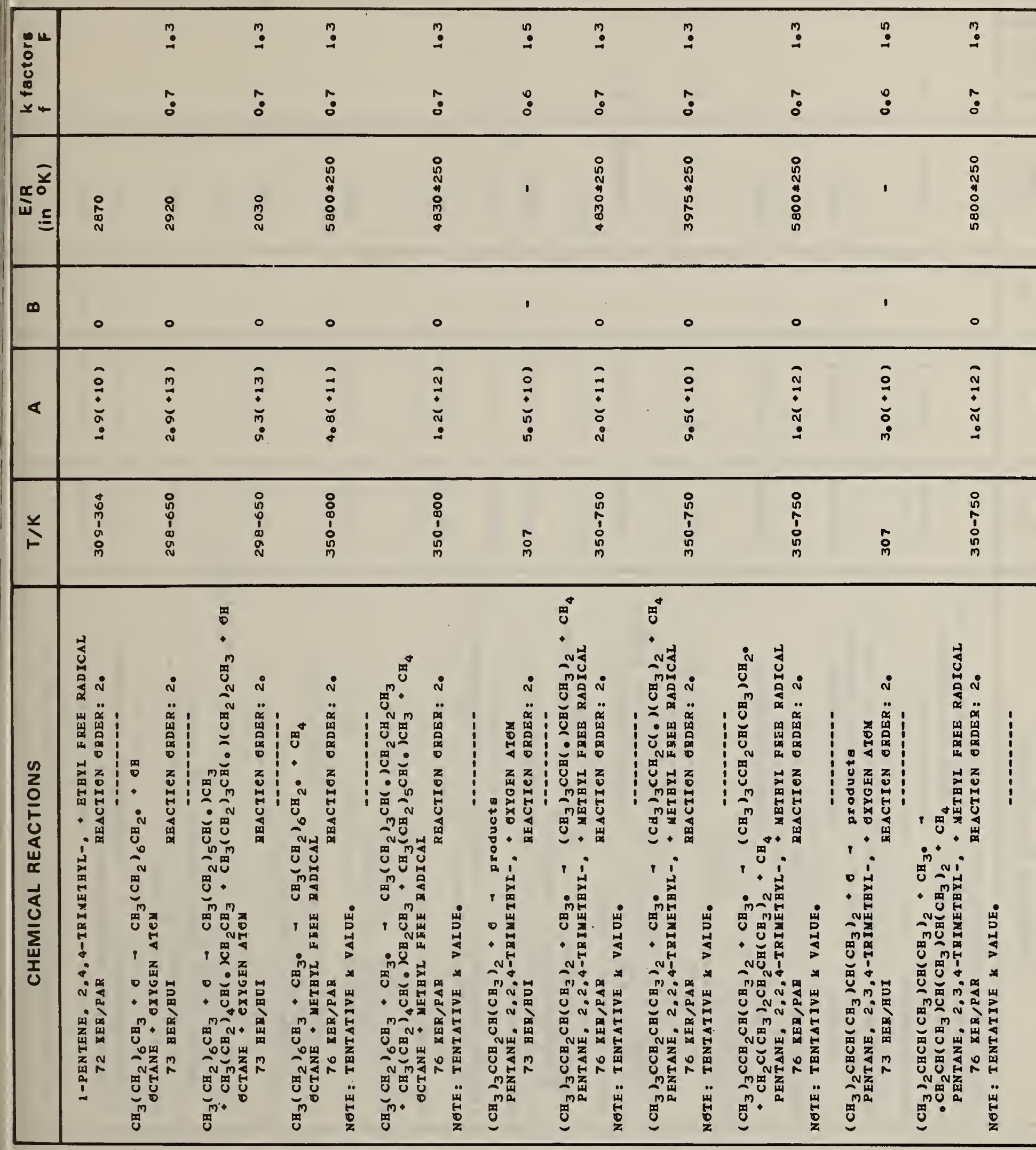




\begin{tabular}{|c|c|c|c|c|c|c|c|c|c|c|c|c|c|c|c|c|c|c|c|c|}
\hline 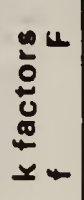 & $\begin{array}{l}\stackrel{m}{:} \\
\text { : }\end{array}$ & 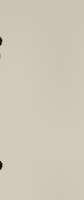 & & & $\begin{array}{l}\stackrel{n}{:} \\
:\end{array}$ & $\begin{array}{l}\text { ?: } \\
\text { : }\end{array}$ & & & & & & & & & & & & & & \\
\hline 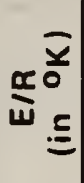 & 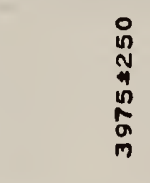 & & 足 & $\begin{array}{l}\stackrel{\vdots}{0} \\
0 \\
m \\
m\end{array}$ & ' & $\begin{array}{c}8 \\
\vdots \\
\vdots \\
\vdots \\
\vdots\end{array}$ & & & $\begin{array}{l}\stackrel{\vdots}{\circ} \\
\stackrel{0}{0}\end{array}$ & & 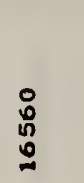 & & 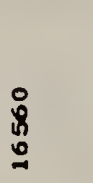 & & 吕 & & $\begin{array}{l}\stackrel{\vdots}{\$} \\
\stackrel{\alpha}{\sigma}\end{array}$ & & 总 & \\
\hline$\infty$ & 0 & & ○ & 0 & ' & 。 & & & ० & & 0 & & 0 & & - & & 0 & & 0 & \\
\hline$<$ & $\begin{array}{l}\bar{\vdots} \\
\vdots \\
\vdots \\
\dot{\alpha}\end{array}$ & & $\underset{\dot{\vdots}}{\dot{\vdots}}$ & $\begin{array}{l}\vdots \\
\vdots \\
\vdots \\
\vdots \\
\vdots\end{array}$ & $\begin{array}{l}a \\
\vdots \\
\dot{0}\end{array}$ & $\vdots$ & & & $\bar{\Xi}$ & & $\begin{array}{l}0 \\
\vdots \\
\vdots \\
\dot{m}\end{array}$ & & $\begin{array}{l}0 \\
\vdots \\
\vdots \\
\vdots \\
\vdots \\
0\end{array}$ & & $\begin{array}{l}5 \\
\vdots \\
\vdots \\
\vdots \\
\vdots\end{array}$ & & $\stackrel{m}{\vdots}$ & & $\begin{array}{l}\vdots \\
\vdots \\
\vdots \\
\vdots\end{array}$ & \\
\hline 呙 & 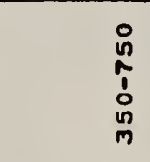 & & $\begin{array}{l}\text { : } \\
\vdots \\
\vdots \\
\vdots \\
0\end{array}$ & 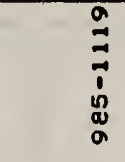 & 吕 & 离 & & & 恿 & & 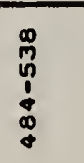 & & 离 & & 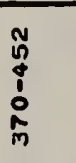 & & $\begin{array}{l}0 \\
\stackrel{0}{0} \\
\vdots \\
:\end{array}$ & & 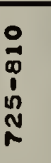 & \\
\hline 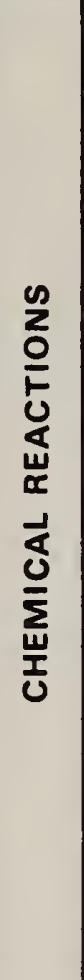 & 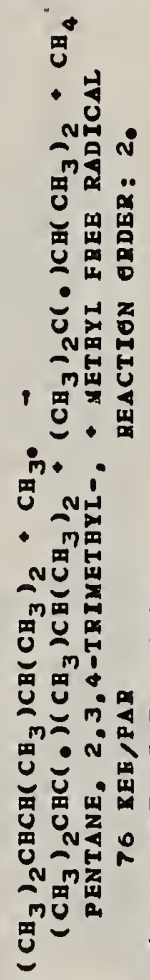 & 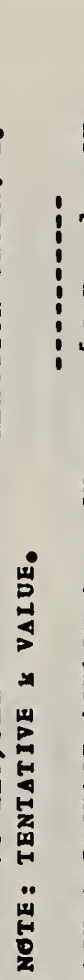 & 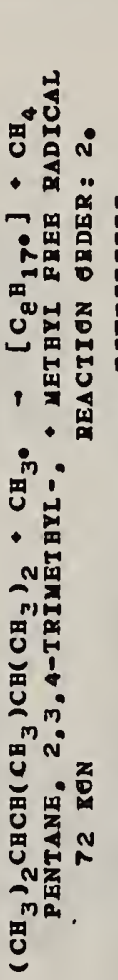 & 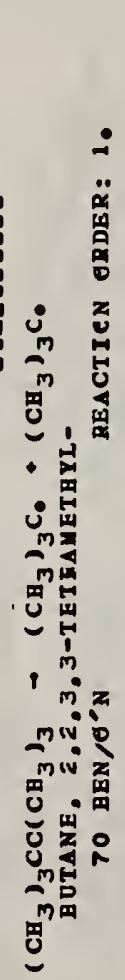 & 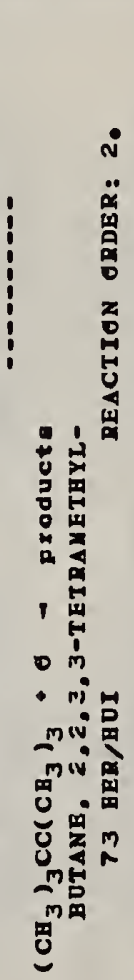 & 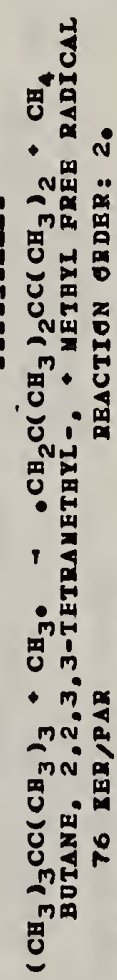 & 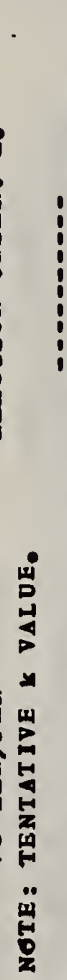 & 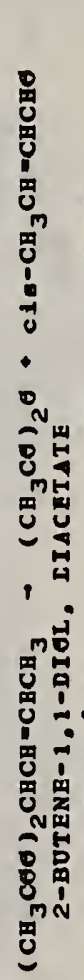 & 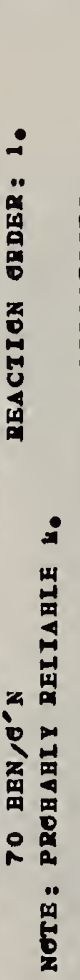 & 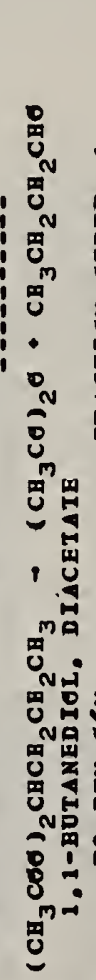 & 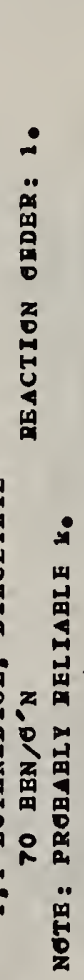 & 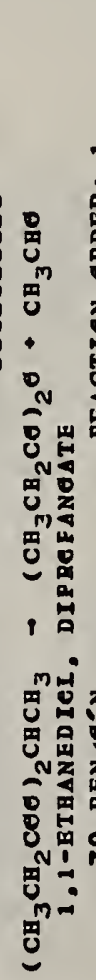 & 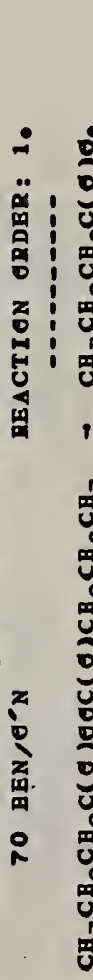 & 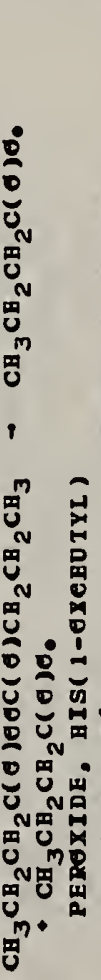 & 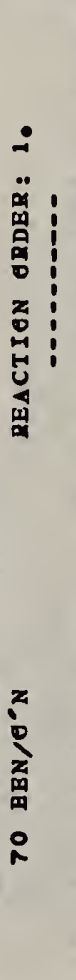 & 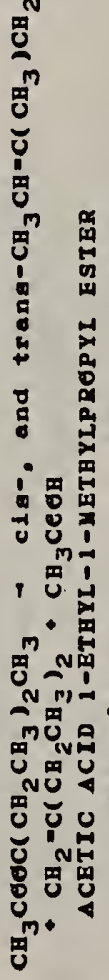 & 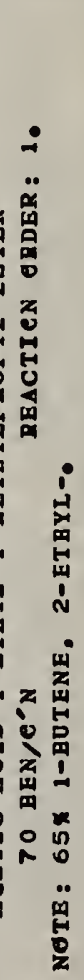 & 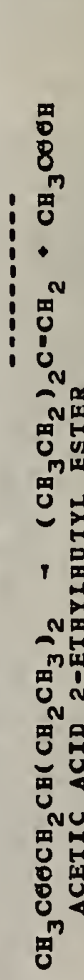 & 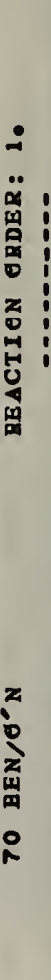 & 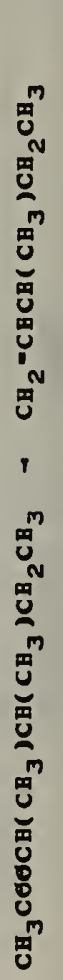 \\
\hline
\end{tabular}




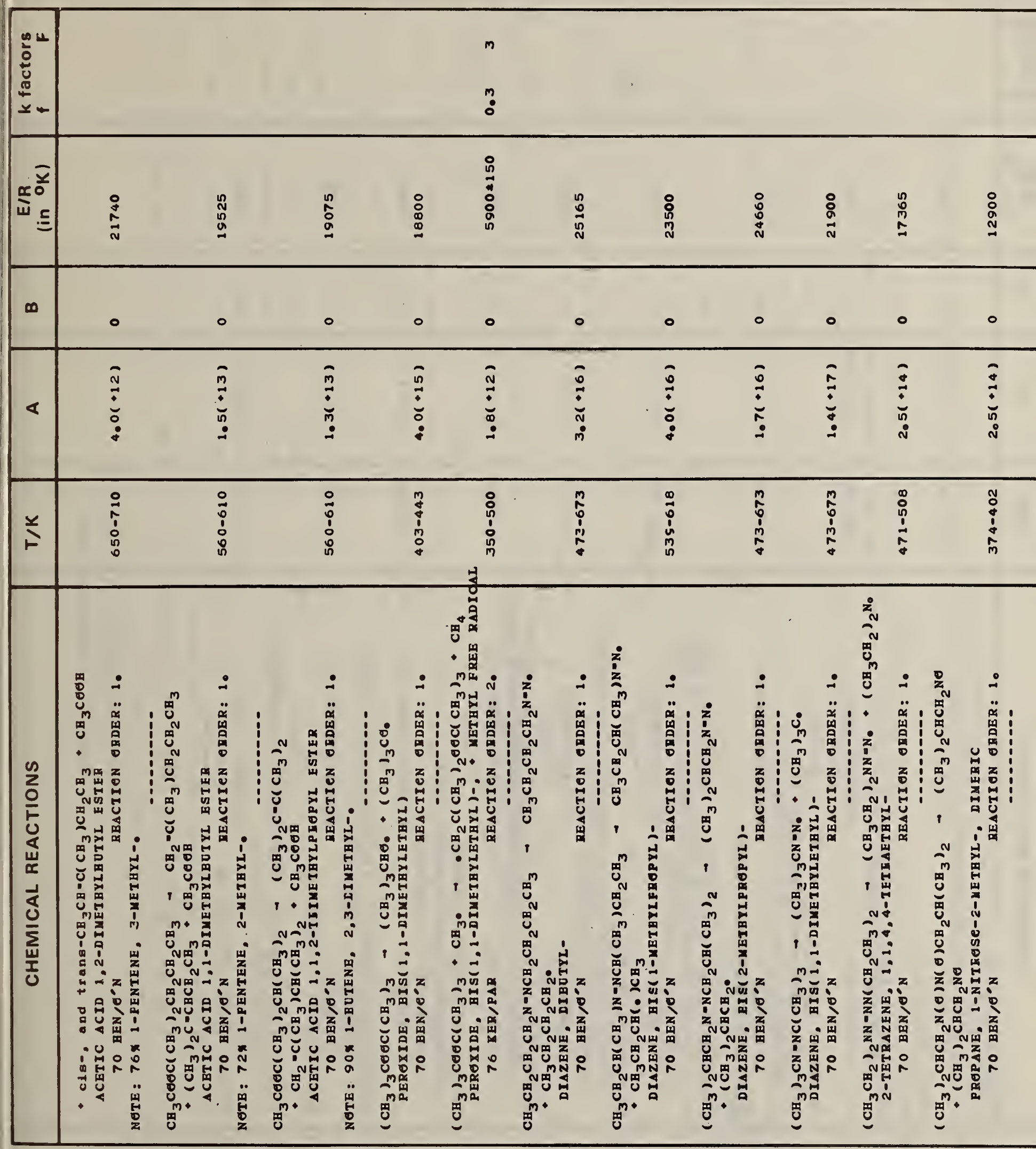




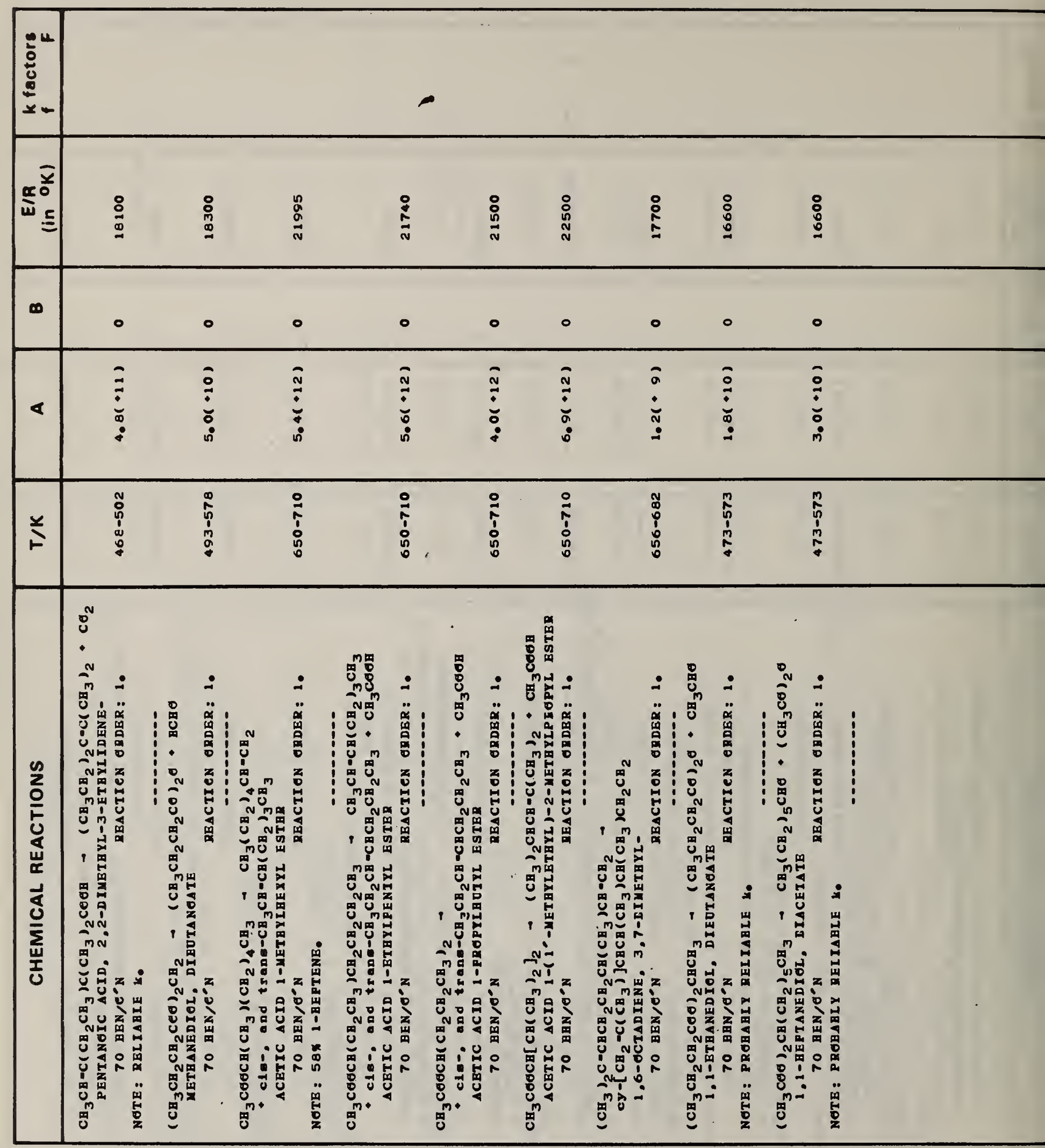




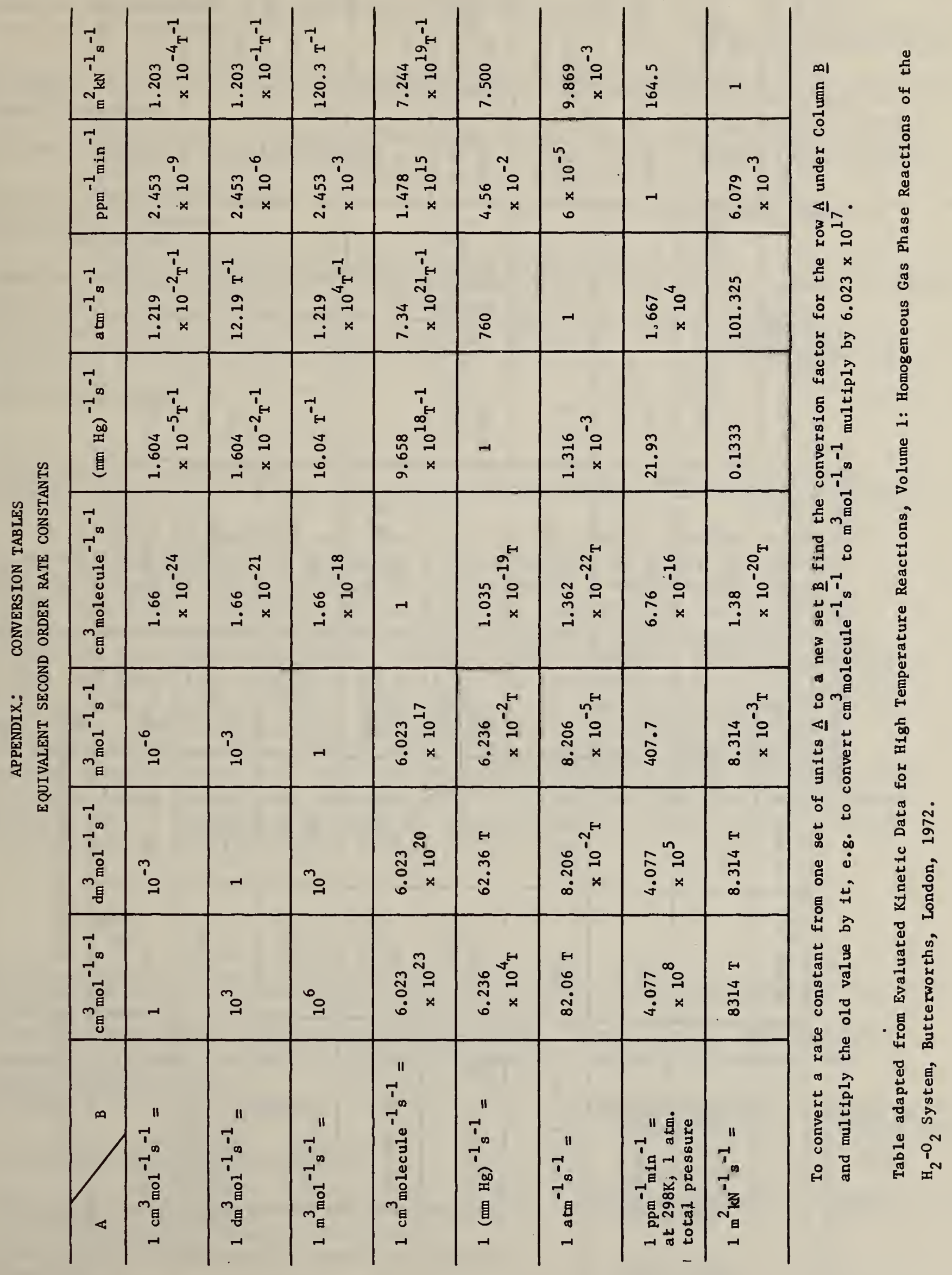




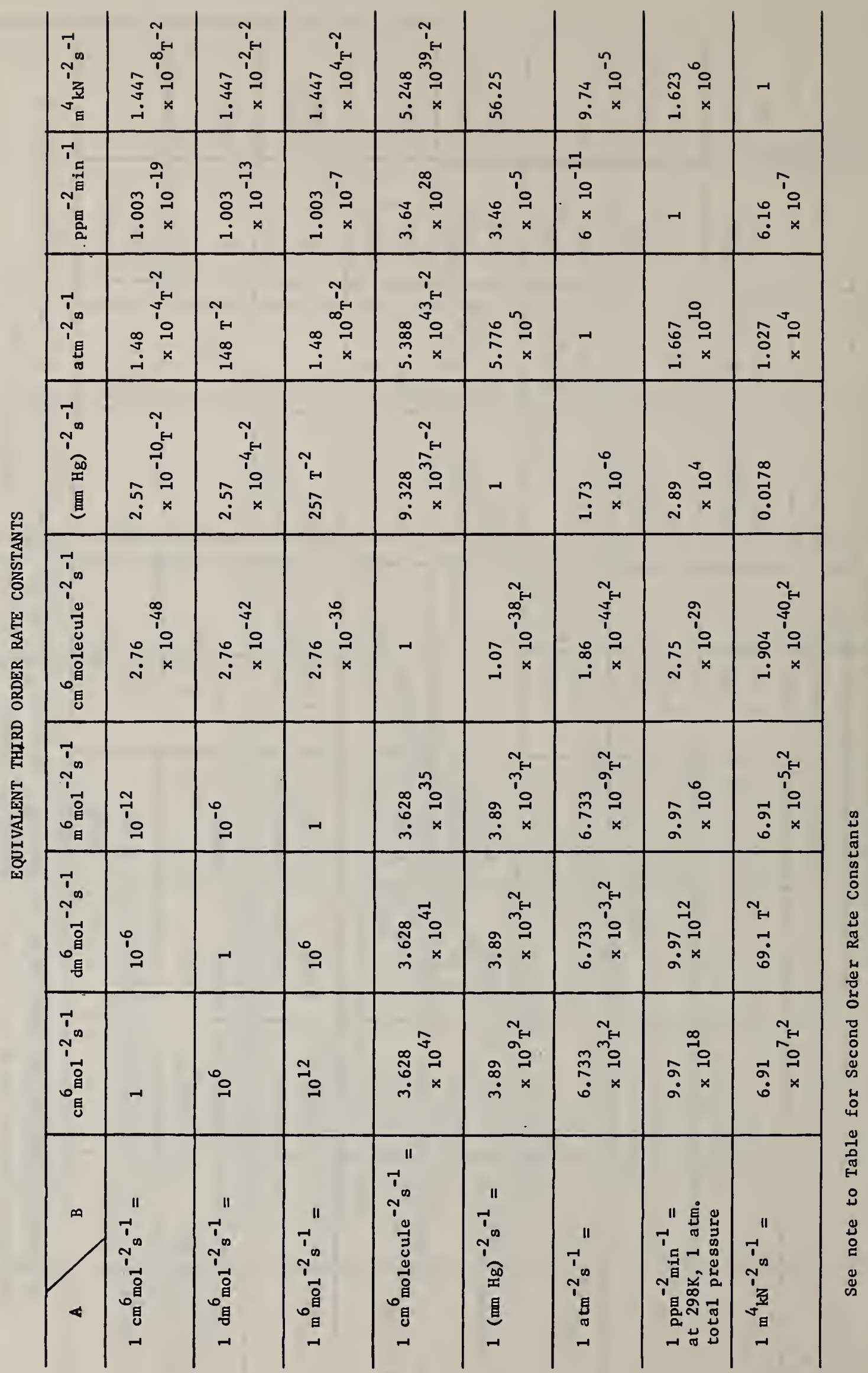


4. TITLE AND SUBTITLE

Table of recommended rate constaris for chemical reactions occurring in combustion
5. Publication Date

Nov. 1979

\section{AUTHOR(S) \\ Francis Westiey \\ 9. PERFORMING ORGANIZATION NAME AND ADDRESS \\ NATIONAL BUREAU OF STANDARDS \\ DEPARTMENT OF COMMERCE \\ WASHINGTON, DC 20234}

12. SPONSORING ORGANIZATION NAME AND COMPLETE ADDRESS (Street, CIT,. State, ZIP)

Department of Energy, Washington, DC 20545 and Office of Standard Reference Data, National Bureau of Standards, Washington, DC 20234
6. Performing O'zanization Code

8. Performing Organ. Report No.

EA-77-A-01-0610

1a. Project Task/Fiork Unit No.

11. Contract/Grant No.

13. Type of Report \& Period Covered

Final

$1950-1975$

14. Sponsoring Agency Code

15. SUPPLEMENTARY NOTES

Document describes a computer program; SF-185, FIPS Software Summary, is attached.

16. ABSTRACT (A 200-word or lose lactual sumery of most oignificant information. If document includea a agnilicant bibliography or lilersture survey, unention it here.)

A table of recommended rate constants for gas phase chemical reactions occurring in combustion is presented. Specifically, it gives in tabular form the values of the parameters for the modified Arrhenius equation $k=A T^{B} \exp (-E / R T)$. The table covers reactions occurring in the combustion, oxidation and decomposition of aliphatic saturated or unsaturated $C_{1}$ to $C_{10}$ hydrocarbons, alcohols, aldehydes, ketones, thiols, ethers, peroxides, amines, amides and their free radicals, as well as the reactions of $0, \mathrm{O}_{2}, \mathrm{H}, \mathrm{H}_{2}, \mathrm{OH}$, $\mathrm{H}_{2} \mathrm{O}, \mathrm{H}_{2} \mathrm{O}_{2}, \mathrm{~N}, \mathrm{~N}_{2}, \mathrm{NO}, \mathrm{N}_{2} \mathrm{O}, \mathrm{NO}_{2}, \mathrm{~N}_{2} \mathrm{O}_{4}, \mathrm{~N}_{2} \mathrm{O}_{5}, \mathrm{~S}, \mathrm{~S}_{2}, \mathrm{SH}, \mathrm{SO}, \mathrm{SO}_{2}, \mathrm{SOH}$, NS, with each other. The table includés approximately $170^{\circ}$ first order reactions 760 second order reactions and 50 third order reactions. There are 1805 entries covering about 1100 distinct chemical reactions. These recommendations have been taken from eleven evaluations and critical reviews published between 1970 and 1976. The papers examined by the evaluators extend from the nineteen fifties up to - and including - 1975.

17. KEY WORDS (aix to welte entries; Aphabetical order; cepicalize only the lisel letter of the lirat key word unleat a proper name; eeparased by cemicolono)

Arrhenius parameters; combustion; decomposition; free radicals; hydrocarbons; hydrogen; nitrogen; oxidation; oxygen; oxygenated; organic compounds; rate constant; sulfur

18. AVAILABILITY DX, Unlimited

For Official Distribution. Do Ilot Release to NTIS

Order From Sup. of DoC., U.S. Govemment Printing Office, Washington, DC 20402, SD Stock No. SNOO3-003-

- Order From Natıonal Technical Information Service (NTIS), Springfield, VA. 22161

\begin{tabular}{|l|c|}
\hline $\begin{array}{l}\text { 19. SECURITY CLASS } \\
\text { (THIS REPORT) }\end{array}$ & $\begin{array}{c}\text { 21. NO. OF } \\
\text { PRINTED PAGES } \\
169\end{array}$ \\
UNCLASSIFIED & \\
\hline $\begin{array}{l}\text { 20. SECURITY CLASS } \\
\text { (THIS PAGE) }\end{array}$ & 22. PIICE \\
UNCLASSIFIED & \\
\hline
\end{tabular}


